\title{
Optoelectronic Device Simulations based on Macroscopic Maxwell-Bloch Equations
}

\author{
Christian Jirauschek@a) Michael Riesch 0 , and Petar Tzenov \\ Department of Electrical and Computer Engineering, Technical University of Munich, Arcisstr. 21, 80333 Munich, \\ Germany
}

(Dated: 9 June 2020, published as Adv. Theory Simul. 2, 1900018 (2019))

Due to their intuitiveness, flexibility and relative numerical efficiency, the macroscopic Maxwell-Bloch (MB) equations are a widely used semiclassical and semi-phenomenological model to describe optical propagation and coherent light-matter interaction in media consisting of discrete-level quantum systems. This review focuses on the application of this model to advanced optoelectronic devices, such as quantum cascade and quantum dot lasers. The Bloch equations are here treated as a density matrix model for driven quantum systems with two or multiple discrete energy levels, where dissipation is included by Lindblad terms. Furthermore, the one-dimensional MB equations for semiconductor waveguide structures and optical fibers are rigorously derived. Special analytical solutions and suitable numerical methods are presented. Due to the importance of the MB equations in computational electrodynamics, an emphasis is placed on the comparison of different numerical schemes, both with and without the rotating wave approximation. The implementation of additional effects which can become relevant in semiconductor structures, such as spatial hole burning, inhomogeneous broadening and local-field corrections, is discussed. Finally, links to microscopic models and suitable extensions of the Lindblad formalism are briefly addressed.

Keywords: Maxwell-Bloch equations, Lindblad equation, Quantum dots, Quantum cascade laser, Waveguide propagation

This is the peer reviewed version of the following article: C. Jirauschek, M. Riesch, and P. Tzenov, "Optoelectronic device simulations based on macroscopic Maxwell-Bloch equations", Adv. Theory Simul. 2, 1900018 (2019), which has been published in final form at https://doi.org/10.1002/adts.201900018. This article may be used for non-commercial purposes in accordance with Wiley Terms and Conditions for Use of Self-Archived Versions.

\section{CONTENTS}

List of Symbols and Acronyms

\section{Introduction}

II. Lindblad equation

A. Introduction of Basis States

B. Choice of Lindblad Operators

1. Incoherent Transitions

2. Pure Dephasing

3. General Case

C. Conditions for Validity

III. Optical Bloch Equations

A. Dipole Approximation

B. Optical Bloch Equations in Standard Form

C. Optical Dipole Matrix Element

1. Computation of Envelope

Wavefunction

2. Inter- and Intraband Dipole Matrix Elements

12

9

10

10

11
D. Non-Redundant Density Matrix Representation

13

E. Rotating Wave Approximation

14

IV. Maxwell-Bloch Equations

A. Macroscopic Polarization And Current Density

1. Coherent Contribution

2. Incoherent Contribution

B. Slowly Varying Amplitude

Approximation

1. Polarization in Rotating Wave

Approximation

C. Initial Conditions

D. Two-Level Approximation

1. Rotating Wave/Slowly Varying

15

15

16

17

17

18

18

19

\footnotetext{
a)Electronic mail: jirauschek@tum.de
} Amplitude Approximation

V. Reduction to One-Dimensional Model

A. Full Maxwell Equations

1. Transverse Electric Mode

2. Transverse Magnetic Mode

B. Slowly Varying Amplitude Approximation

20

C. Fabry-Pérot Type Resonator

1. Boundary Conditions at the End Facets
20

20

22

23

25

26

27 
2. Spatial Hole Burning

VI. Analytical Solutions

A. Rotating Wave Approximation

1. Monochromatic Excitation

2. Self-Induced Transparency

B. Full-Wave Bloch Equations

VII. Numerical Schemes

A. Rotating Wave/Slowly Varying Amplitude

Approximation

1. Finite Difference Discretization of the One-Dimensional Propagation Equation

2. Density Matrix Equations

3. Generalizations and Alternative Methods

B. Full-Wave Simulation

1. Numerical Schemes for Maxwell's Equations

2. Coupling Electric Field Updates and Density Matrix Updates

3. Crank-Nicolson Scheme/Predictor-Corrector Method

4. Runge-Kutta Method

5. Matrix Exponential Methods

6. Comparison of Numerical Methods for the Bloch Equations

7. Alternative Methods

VIII. Inclusion of Further Effects

A. Local-Field Correction

1. Near-Dipole-Dipole Effects in Dense Media

2. Depolarization Field in Tightly Localized Quantum Systems

B. Inhomogeneous Broadening

C. Noise

IX. Application to Optoelectronic Devices

A. Bulk and Quantum Well Interband Optoelectronic Devices

B. Quantum Well Intersubband Devices

C. Quantum Dot Devices

X. Conclusion and Outlook

Conflicts of Interest

Supporting Information

Acknowledgments

Biographies

\section{LIST OF SYMBOLS AND ACRONYMS}

Symbol Description

\begin{tabular}{|l|}
29 \\
\hline 29 \\
\hline 29 \\
\hline 32 \\
\hline 33 \\
\hline
\end{tabular}

33

A Magnetic vector potential

$A_{\mathrm{cv}} \quad$ Spontaneous emission coefficient

$A_{\text {eff }} \quad$ Effective mode area

$A_{\mathrm{q}} \quad$ Quantum active region cross section

a Power loss coefficient

c Vacuum speed of light

$D \quad$ Diffusion coefficient

D Displacement field

$\mathcal{D} \quad$ Dissipation superoperator

$\mathcal{D}_{i j m n}$ Tensor element of $\mathcal{D}$

$\hat{\mathbf{d}} \quad$ Dipole operator

$\mathbf{d}_{i j} \quad$ Dipole matrix element vector

E Electric field

$\underline{\mathbf{E}}$ Slowly varying amplitude of $\mathbf{E}$

$\underline{E} \quad \underline{E}=\underline{\mathbf{E e}}$

$\underline{E}_{ \pm} \quad \underline{E}$ for forward/backward propagating field

$E_{\mathrm{g}} \quad$ Bandgap energy

$E_{i} \quad$ Eigenenergy of level $i$

$\underline{E}_{p}^{\omega} \quad$ Fourier amplitude of waveguide E-field

$\underline{\mathbf{E}}^{p} \quad$ Transverse dependency of $\underline{\mathbf{E}}$

EIT Electromagnetically induced transparency

$e \quad$ Elementary charge

e Polarization direction of electric field

$F \quad$ Modal field distribution

$F_{i} \quad$ Wavefunction of semiconductor state $i, F_{i}=\varphi_{i} u_{v_{i}}$

FDTD Finite difference time-domain

$f^{ \pm} \quad$ Forward/backward normalized polarization amplitude

$g \quad$ Power gain coefficient

$g(\omega) \quad$ Distribution function of resonance frequencies

H Magnetic field

$\underline{\mathbf{H}} \quad$ Slowly varying amplitude of $\mathbf{H}$

$\overline{\hat{H}} \quad$ Hamiltonian

$\hat{H}_{0} \quad$ Unperturbed system Hamiltonian

$\hat{H}_{1} \quad$ Interaction Hamiltonian

$\underline{H}_{p}^{\omega} \quad$ Fourier amplitude of waveguide $\mathrm{H}$-field

$\underline{\mathbf{H}}^{\mathrm{t}} \quad$ Transverse dependency of $\underline{\mathbf{H}}$

$\bar{\hbar} \quad$ Reduced Planck constant

39

40

I Optical intensity

$I_{\mathrm{S}} \quad$ Saturation intensity

$\mathbf{J}_{\mathrm{f}} \quad$ Current density due to free carriers

$\mathbf{J}_{\mathrm{q}} \quad$ Total current density due to quantum systems

k $3 \mathrm{D}$ bulk/2D in-plane wavevector

$k_{0} \quad \omega / c$

$k_{\mathrm{c}} \quad$ Carrier wavenumber with $\left|k_{\mathrm{c}}\right|=n_{0} \omega_{\mathrm{c}} / c$

$\mathcal{L} \quad$ Liouville superoperator

$\hat{L} \quad$ Lindblad operator

$\hat{L}_{\alpha \rightarrow \beta} \quad \hat{L}$ for incoherent transition

$\ell \quad v_{\mathrm{g}} a / 2$

MB Maxwell-Bloch

$m \quad$ Mass

$m^{*} \quad$ Effective mass

48

$m_{\mathrm{e}} \quad$ Electron mass

$N \quad$ Number of system levels

$\underline{n} \quad$ Complex refractive index

$n_{0} \quad$ Background refractive index

$53 \quad n_{2 \mathrm{D}} \quad$ QD sheet density

$n_{3 \mathrm{D}} \quad$ Carrier number density

$n_{\mathrm{cv}} \quad$ Number density of electron-hole pairs

$n_{\text {eff }} \quad$ Effective waveguide index $\beta / k_{0}$

$\underline{n}_{\text {eff }} \quad$ Complex effective waveguide index $\underline{\beta} / k_{0}$

$P \quad$ Optical power

P Macroscopic polarization 


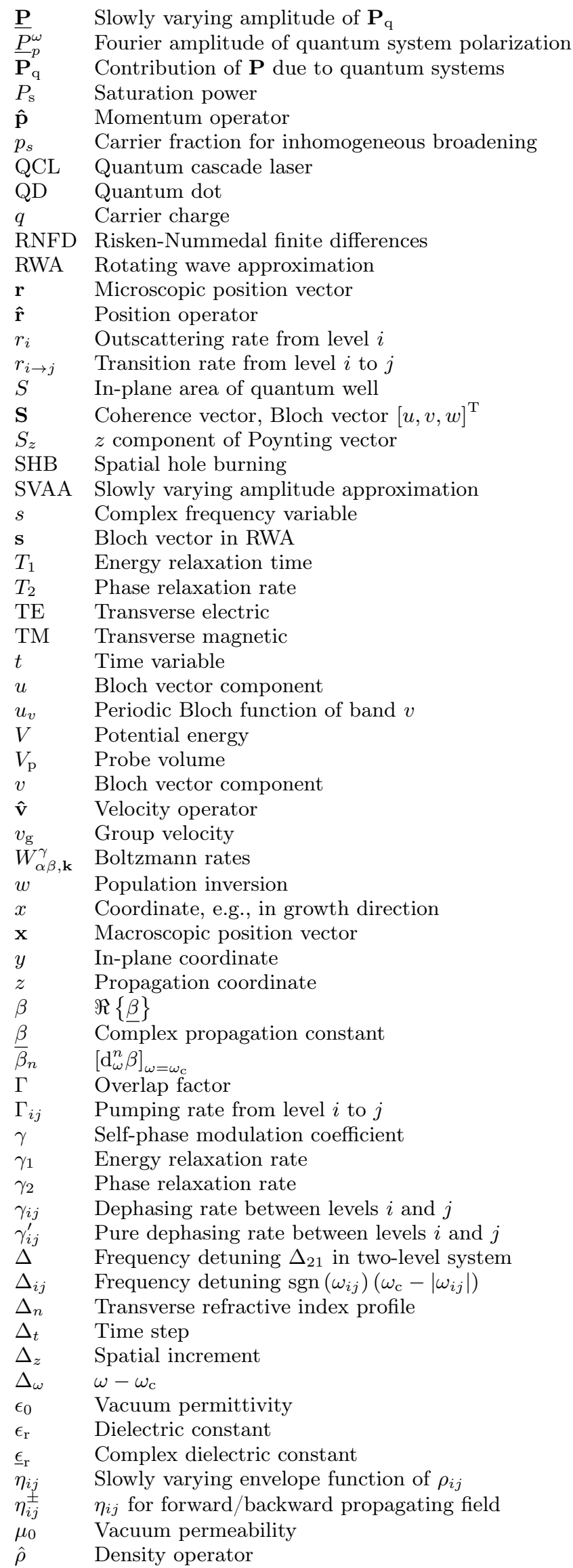

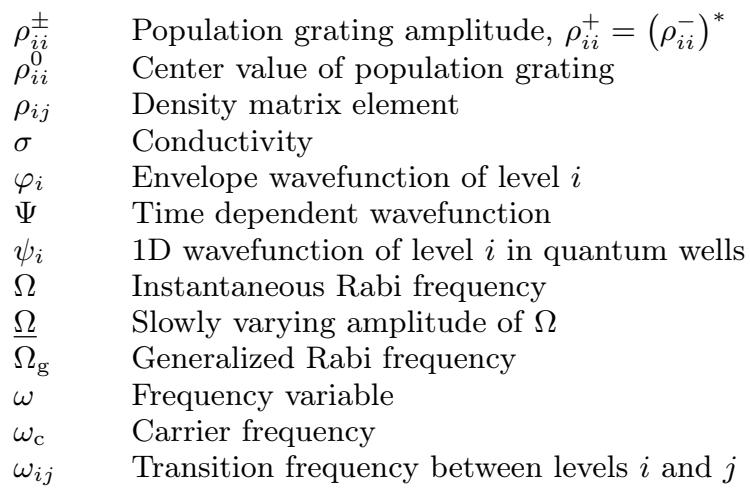

\section{INTRODUCTION}

Due to advancements in nanotechnology, structuring in the nanometer range is meanwhile routinely exploited in electronics and photonics. For example, in optoelectronic devices such as semiconductor optical amplifiers and lasers, quantum confinement is widely used to concentrate the carriers in certain energy states, yielding improved wall-plug efficiencies and higher output powers. As a further effect, the wavelength can be tuned by changing the size of the confinement structure. On a commercial basis, mostly one-dimensional confinement is used in form of quantum well structures, which are fabricated based on deposition of nanometer-thin semiconductor layers of different compositions. In such structures, a quantum well is formed by a layer consisting of a lower bandgap material than the adjacent layers, which restricts the free electron motion in that layer to the inplane directions and gives rise to quantized energy states in growth direction. As a consequence of the further restriction of the energy spectrum and the even stronger carrier localization, additional improvement can be expected from two- or three-dimensional confinement, resulting in quantum wire/dash and quantum dot (QD) structures, respectively. Indeed, $\mathrm{QD}^{1+3}$ and quantum dash $^{4}$ lasers and laser amplifiers have been shown to exhibit excellent characteristics. In Fig. 1, the formation of quantized states in quantum wells, wires and dots is schematically illustrated. The term quantum dash refers to an elongated nanostructure, i.e., some kind of short quantum wire. By contrast, the term nanowire does not necessarily indicate strong quantum confinement. For example, in nanowire lasers the nanowire geometry typically serves as a single-mode optical waveguide resonator, while the active region is based on a heterostructure or quantum well, as in a conventional laser diode ${ }^{516}$

Semiconductor optoelectronic devices usually rely on electron-hole recombination, i.e., optical transitions between conduction and valence band states. The associated resonance wavelength is largely determined by the semiconductor bandgap, which establishes a lower bound on the transition energy. Thus, the coverage of a certain spectral region depends on the existence of suitable semiconductor materials, which for example restricts the availability of practical optoelectronic sources and de- 

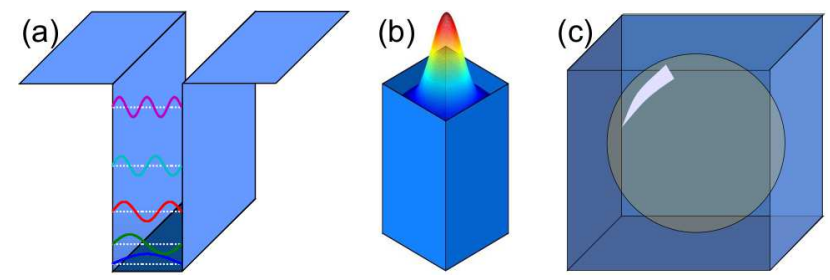

FIG. 1. Schematic illustration of quantum confinement structures: (a) Quantum well, (b) quantum wire, (c) quantum dot.

tectors in the mid-infrared and terahertz regions. An alternative concept is based on intersubband devices, which employ so-called intersubband transitions between quantized energy states in the conduction (or, in some cases, valence) band of a nanostructure, and thus allow quantum engineering of the transition wavelength independent of the bandgap. Quantum well devices based on this concept include quantum cascade lasers, ${ }^{7}$ quantum cascade detectors 899 and quantum well infrared photodetectors. ${ }^{10}$ Furthermore, intersubband transitions are used for QD infrared photodetectors $11-13$

Along with quantum confinement, also quantum coherent effects are found to be increasingly relevant for modern optoelectronic devices. Such effects result from the coherent light-matter interaction, which requires that the states involved in the optical transition maintain a well-defined phase relationship over a significant time. The coherent interaction manifests itself in so-called Rabi flopping, 14 i.e., carrier population oscillations between the states, which are driven by the optical field. The resulting carrier dynamics couples back to the optical field via the polarization, thus also affecting the propagating optical waveform. Besides being an essential prerequisite for the emerging field of quantum information technology, 15 quantum coherence plays an increasingly important role for modern optoelectronic devices in general. Due to the strong interaction with the semiconductor environment, e.g., in the form of phonon scattering and carrier-carrier interactions, this phase relationship tends to be quickly destroyed, which is commonly referred to as dephasing. However, under favorable conditions, signatures of Rabi oscillations have been observed in nanostructured optoelectronic systems and devices. These include quantum well structures, 1617 nanowire lasers, $\frac{6}{6}$ quantum cascade lasers, $\frac{18}{18}$ and single QDs ${ }^{1920}$ at cryogenic temperatures, as well as $\mathrm{QD}^{21 / 22}$ and quantum dash 23 amplifiers at room temperature. Closely related is self-induced transparency, ${ }^{[2 \mid 25}$ where Rabi flopping enables a special optical pulse form to propagate without being attenuated or disturbed. This phenomenon has meanwhile also been observed in semiconductor structures such as QD waveguides, $\frac{22 \mid 26]}{2}$ with potential applications such as the generation of ultrashort optical pulses in QD and quantum cascade lasers.27 31 Another effect that relies on quantum coherence is slow light propagation or even complete halting of light, 32 with possible applications such as optical buffers,, 35 imaging and quantum memory $\stackrel{38}{[}$ This effect has meanwhile also been demonstrated in solid-state media, namely in doped crystals. $\frac{3940}{40}$ The use of suitably engineered semiconductor structures would be especially attractive from a practical point of view 4143 Furthermore, quantum interference, e.g., in QD or intersubband quantum well systems, is an interesting candidate to realize all-optical switching. $44 \sqrt[45]{ }$ Due to the discrete energy level structure of QDs, semiconductor devices based thereupon are especially likely to be, at least in part, governed by coherence effects, $\frac{15}{15}$ although for QD ensembles the dephasing tends to be strong. ${ }^{21}$ The same applies to intersubband quantum well devices, where the levels close to the band edge have parallel dispersion relations, and thus the quantum dynamics resembles that of discrete-level systems 18 Especially in such devices, coherence effects can significantly influence the dynamic operation even for considerable dephasing.

Suitable theoretical models are required for an in-depth understanding of the often quite complex interplay of effects determining the dynamic device characteristics, as well as for quantitative simulation and systematic device optimization. For the optoelectronic devices and structures discussed above, an adequate theoretical description must include the coherent carrier dynamics, incoherent processes such as scattering or spontaneous emission, as well as the interaction with the optical field. Our focus is here on the very widely used Maxwell-Bloch (MB) equations. The Bloch equations provide a compact model for the discrete-level carrier dynamics, which is described by the density matrix formalism. The coherent singlecarrier dynamics is here modeled by the Hamiltonian of the quantum system, such as a QD, and also includes the interaction with a classical optical field. Effects beyond the single-electron quantum evolution are regarded as interaction with the environment in form of the semiconductor host, which gives rise to incoherent effects such as scattering with other carriers and phonons. The resulting dissipation in the quantum system is in the Bloch equations phenomenologically modeled by relaxation rate terms, which introduce dephasing and incoherent carrier transitions. The Bloch equations were first devised to describe the evolution of the nuclear magnetic moment in a magnetic field, $\frac{46}{4}$ and later on extended to a pair of levels in resonance with a classical optical field! $\stackrel{47 \mid 48}{ }$ The model is closed by coupling the Bloch equations to Maxwell's equations, 24/47/49 which describe the evolution of the classical optical field. This review paper is concerned with the resulting $\mathrm{MB}$ equations, where we go beyond the often applied two-level approximation $24 \mid 4749$ by considering multiple, albeit discrete, energy levels. Furthermore, we root the phenomenological dissipation terms in the Lindblad formalism, which ensures physical behavior of the quantum system and allows for the construction of more general dissipation terms.

The MB equations offer a generic description of semiclassical light-matter interaction, which can be applied to different media such as semiconductor structures or 
gases. The focus of this review lies on semiconductor structures, which is reflected in the treatment of some specific issues, such as the concrete embodiment of Maxwell's equations, or the inclusion of spatial hole burning in linear resonators. Independent of the modeled system, the main attractiveness of the $\mathrm{MB}$ equations lies in the relatively compact description of the carrier dynamics, which is helpful for providing intuitive insight into the device behavior and even allows for closed analytical solutions in some special cases ${ }^{[24 \mid 49}$ From a computational point of view, the Bloch equations are widely used in combination with electromagnetic simulations, e.g., based on the finite-difference time-domain method, as a quantum model of the medium, $\frac{52]}{15}$ replacing simpler classical descriptions such as the Lorentz model. Due to the relative compactness of the Bloch model, also computationally demanding two- or three-dimensional simulations can be carried out ${ }^{53}[60$ Likewise, the MB equations enable systematic device optimizations over a large parameter range, as well as long-term simulations, e.g., to investigate the steady-state laser dynamics $\frac{2916162}{\mathrm{An}}$ other important advantage of the MB equations is that they can easily be adapted to specific problems by adding further effects, such as inhomogeneous broadening $24 \mid 49$ or local-field corrections $63[64$

Clearly, the Bloch equations constitute a compromise between accuracy and compactness of the model. A full microscopic treatment of light-matter interaction in a semiconductor, accounting for carrier-phonon and manybody Coulomb interactions as well as for free carrier motion in the unconfined directions, results in the so-called semiconductor MB equations $[6566$ These do not require phenomenological input parameters, but the significantly increased model complexity usually restricts the modeling to one spatial dimension and short-term simulations. While the semiconductor $\mathrm{MB}$ equations are beyond the scope of this review, they can be used as a basis to derive macroscopic discrete-level MB equations with Lindblad dissipation and additional correction terms for specific semiconductor structures $[67 \sqrt[69]{69}$

In detail, our paper is organized as follows: In Section III the density matrix formalism and Lindblad model are introduced, serving as a basis for the Bloch equations. These are treated in Section III which also includes a discussion of the widely used rotating wave approximation (RWA). In Section IV, the MB equations are introduced in full-wave treatment and invoking the RWA, along with the slowly varying amplitude approximation (SVAA) for the field propagation. Section $\mathrm{V}$ treats the reduction of the MB equations for semiconductor waveguide structures and optical fibers to a spatially one-dimensional model, which is a widely used simplification. Section VI deals with available analytical solutions for the Bloch and MB equations, while in Section VII numerical methods for the MB equations are covered. Section VIII is dedicated to the inclusion of further effects, such as local-field corrections, inhomogeneous broadening and noise. Section IX deals with the application of the MB model to concrete optoelectronic devices, including bulk as well as inter- and intraband quantum well and QD devices. The paper is concluded in Section $\mathrm{X}$, where dissipation models beyond the Lindblad formalism are discussed.

\section{LINDBLAD EQUATION}

In the following, we consider discrete quantum systems with $N$ states $|i\rangle$, where $i=1 . . N$. We restrict ourselves to a single-particle description, valid for carrier densities which are sufficiently low to neglect Pauli blocking, but sufficiently high to neglect electron-hole Coulomb correlation. ${ }^{70}$ It has been pointed out that these requirements are often fulfilled in state-of-the-art semiconductor quantum devices which are the main scope of this paper, and that the Lindblad approach introduced below is then well justified ${ }^{70}$ Furthermore, we do not explicitly consider spin dependent effects, even though the Lindblad formalism can be extended accordingly ${ }^{71}$

The time evolution of an ideal quantum system is famously described by the time dependent Schrödinger equation

$$
\mathrm{i} \hbar \partial_{t}|\Psi\rangle=\hat{H}|\Psi\rangle
$$

with the reduced Planck constant $\hbar$, where the system state vector $|\Psi\rangle$, and generally also the system Hamiltonian $\hat{H}$, depend on time $t .|\Psi\rangle$ is a pure state, i.e., a coherent superposition of the basis states $|i\rangle$ with $|\Psi(t)\rangle=\sum_{i} c_{i}(t)|i\rangle$, where $c_{i}$ are complex coefficients. In reality, however, no quantum system is perfectly isolated, but rather interacts with its environment. This induces decoherence, i.e., loss of quantum coherence in the system, which must be included into any realistic description. The resulting statistical state of the system is generally a mixed state which cannot be represented by the system state vector $|\Psi\rangle$, but rather requires an extended description in terms of the density operator $\hat{\rho}$. The corresponding density matrix with respect to the chosen basis states $|i\rangle$ has the elements $\rho_{i j}=\langle i|\hat{\rho}| j\rangle$, where the diagonal elements $\rho_{i i}$ give the occupation probability of state $|i\rangle$, while the off-diagonal elements $\rho_{i j}$ represent the coherence between $|i\rangle$ and $|j\rangle$. The density operator is positive semidefinite which guarantees that any pure system state $|\Psi\rangle$ has a non-negative probability, i.e., $\langle\Psi|\hat{\rho}| \Psi\rangle \geq 0$. This also implies Hermiticity, i.e., $\hat{\rho}=\hat{\rho}^{\dagger}$ and thus $\rho_{i j}=\rho_{j i}^{*}$, where the dagger and asterisk denote the adjoint and the complex conjugate, respectively. Furthermore, at least for closed systems, ${ }^{72}$ the trace must remain constant to ensure particle conservation and is usually normalized to unity, $\operatorname{Tr}\{\hat{\rho}\}=1$. The coherent time evolution of the density operator is in the Schrödinger picture described by the von Neumann equation

$$
\mathrm{i} \hbar \partial_{t} \hat{\rho}=[\hat{H}, \hat{\rho}] .
$$




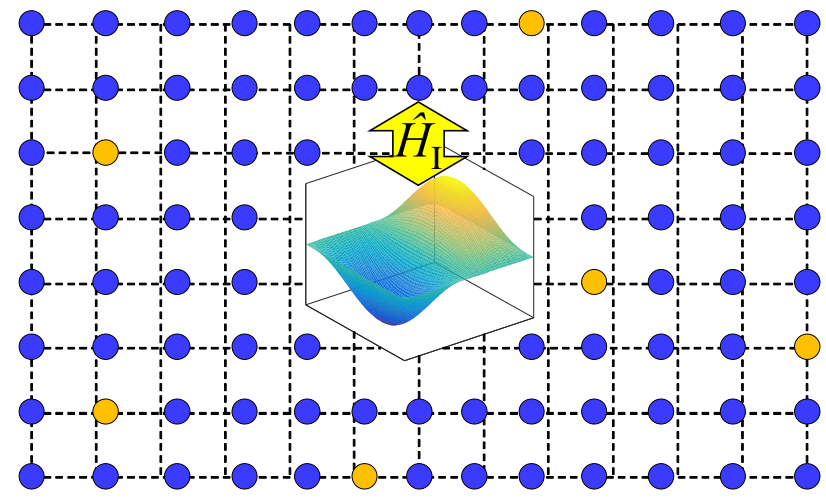

FIG. 2. Schematic illustration of a quantum system interacting with impurities and thermal vibrations in the semiconductor lattice.

In realistic scenarios, often many degrees of freedom are relevant for the time evolution and must thus be considered. Usually, only part of these degrees of freedom are of direct interest for the application in mind, and solving the full Eq. 22) is typically also too demanding. This issue can be addressed by performing a division into a system containing the degrees of freedom which are of primary interest, and a second one with the remaining degrees of freedom which then constitute the environment. In semiconductor quantum devices, the degrees of freedom of interest may be quantized states in a nanostructure such as a quantum well or dot, while decoherence typically arises from interaction with the semiconductor lattice itself, thus acting as the environment. This situation is schematically illustrated in Fig.2. There are various types of interactions, also referred to as scattering mechanisms, which can induce decoherence in the quantum system of interest. These include the interaction with phonons due to (longitudinal- and transverseoptical and -acoustic) thermal lattice vibrations, lattice imperfections in form of impurities (such as dopants), interface roughness or atomic disorder in alloys, as well as piezoelectric fields. Also carrier-carrier interaction can enter the single-particle picture as an additional scattering mechanism ${ }^{73 \mid 74}$ For a quantum svstem interacting with the environment, $\hat{\rho}$ and $\hat{H}$ in Eq. $(2)$ refer to the full dynamics of the combined system and environment.

The Hamiltonian $\hat{H}$ can be written as $\hat{H}=\hat{H}_{\mathrm{S}} \otimes \hat{I}_{\mathrm{E}}+$ $\hat{I}_{\mathrm{S}} \otimes \hat{H}_{\mathrm{E}}+\hat{H}_{\mathrm{I}}$, where the Hamiltonians $\hat{H}_{\mathrm{S}}, \hat{H}_{\mathrm{E}}$ and $\hat{H}_{\mathrm{I}}$ describe the system $\mathrm{S}$, the environment $\mathrm{E}$ and the systemenvironment interaction, $\hat{I}_{\mathrm{S}}$ and $\hat{I}_{\mathrm{E}}$ are the unit operators in the respective Hilbert spaces, and $\otimes$ denotes the tensor product $\frac{75}{7}$ The reduced density matrix of the system of interest is simply obtained by tracing over the environmental degrees of freedom, $\hat{\rho}_{\mathrm{S}}=\operatorname{Tr}_{\mathrm{E}}\{\hat{\rho}\}$. This step by itself does obviously not eliminate the dependence of Eq. (2) on the environment. Thus, additional assumptions are necessary to arrive at a model for the non-unitary time evolution of $\hat{\rho}_{\mathrm{S}}$, which is a consequence of eliminating the environmental degrees of freedom. The resulting equation is expected to be similar in structure as Eq. (2), i.e., a first-order linear differential equation in time for $\hat{\rho}_{\mathrm{S}}$, where the linearity ensures consistency with the ensemble interpretation of the density matrix ${ }^{76}$ The resulting time-local and Markovian description of the reduced density matrix dynamics is commonly referred to as (quantum) master equation. Its general form can be inferred by posing additional requirements to avoid unphysical behavior. In particular, this includes conservation of unit trace and positive semidefiniteness of the density matrix, as discussed above. Closer inspection reveals that if there exists another system $\mathrm{S}^{\prime}$, an evolution equation for $\mathrm{S}$ which ensures positive semidefiniteness of $\hat{\rho}_{\mathrm{S}}$ can still lead to unphysical time evolution of the combined density matrix for $S$ and $S^{\prime}$, even if $S^{\prime}$ does not evolve and is completely decoupled from $S{ }^{76}$ This problem is cured by demanding complete positivity of the evolution, rather than only the preservation of positive semidefiniteness of $\hat{\rho}_{\mathrm{S}}$.

From above requirements, the general form of the evolution equation can be inferred by invoking the Kraus theorem, ${ }^{77}$ characterizing completely positive trace preserving maps. The resulting master equation is called Lindblad equation. 50151 Dropping the subscript $S$ from here on for ease of notation, it can be written as

$$
\begin{aligned}
\partial_{t} \hat{\rho} & =-\frac{\mathrm{i}}{\hbar}[\hat{H}, \hat{\rho}]+\sum_{k}\left(\hat{L}_{k} \hat{\rho} \hat{L}_{k}^{\dagger}-\frac{1}{2} \hat{L}_{k}^{\dagger} \hat{L}_{k} \hat{\rho}-\frac{1}{2} \hat{\rho} \hat{L}_{k}^{\dagger} \hat{L}_{k}\right) \\
& =\mathcal{L}(\hat{\rho})+\sum_{k} \mathcal{D}_{k}(\hat{\rho})=\mathcal{L}(\hat{\rho})+\mathcal{D}(\hat{\rho}),
\end{aligned}
$$

where $\hat{H}=\hat{H}_{0}+\hat{H}_{1}$ is the effective Hamiltonian of the reduced system. Here, the Hamiltonian $\hat{H}_{1}$ describes externally induced perturbations, e.g., due to an incident optical field. The description of light-matter interaction requires a time dependent Hamiltonian, which, although lifting the originally assumed time-homogeneity of the Lindblad equation, still gives a valid density matrix evolution. $\frac{78 \mid 79}{1 n}$ addition, $\hat{H}$ may contain nondissipative contributions stemming from the interaction with the environment, such as energy shifts. ${ }^{75}$ The dissipation is described by the sum term, where the linear operators $\hat{L}_{k}$ are called Lindblad or (quantum) jump operators, which can in principle be chosen without further restrictions in the Hilbert space of the reduced system. Equation (3) now includes both the coherent dynamics due to the Liouville superoperator $\mathcal{L}(\hat{\rho})$, corresponding to Eq. (2), and the incoherent dynamics induced by the dissipation superoperator $\mathcal{D}(\hat{\rho})$ which contains the interaction with the environment. Besides inferring the Lindblad equation from the requirements given above, Eq. (3) can also be microscopically derived, assuming that the quantum system is weakly coupled to a large Markovian environment. $.75[80181$

As mentioned above, we allow for a time dependent Hamiltonian in Eq. (3), which is required to include lightmatter interaction as envisaged in this paper, and con- 
stitutes a slight generalization of the original equation. 50 Occasionally, also time dependent Lindblad operators $\hat{L}_{k}(t)$ are used, for example to model time dependent pumping rates. ${ }^{82}$ This also does not affect the physical validity of Eq. (3), since conservation of trace and complete positivity are further guaranteed $\frac{78779}{\text { Moreover, }}$ Eq. (3) with time dependent operators $\hat{H}$ and $\hat{L}_{k}$ is still time-local and also Markovian. 83

\section{A. Introduction of Basis States}

In principle, the $N$ basis states of the (reduced) quantum system can be freely selected as long as they span the entire Hilbert space of the $N$-level system. In most cases, an orthonormal basis is the preferred option, since it results in more compact expressions and provides a clearer physical interpretation. The choice of energy eigenstates has the distinct advantage that the reduced system Hamiltonian $\hat{H}_{0}$ is diagonal. In certain cases, other choices may be preferable, such as a localized (or tight-binding) basis set for the description of tunneling, e.g., in double- or multiple-well systems.

Assuming an orthonormal basis so that the unit operator becomes $\hat{I}=\sum_{\ell=1}^{N}|\ell\rangle\langle\ell|$, Eq. $\sqrt{3}$ can be written as

$$
\begin{aligned}
\partial_{t} \rho_{i j}= & -\frac{\mathrm{i}}{\hbar} \sum_{\ell}\left(H_{i \ell} \rho_{\ell j}-H_{\ell j} \rho_{i \ell}\right) \\
& +\sum_{k} \sum_{\ell p}\left[L_{k}^{i \ell}\left(L_{k}^{j p}\right)^{*} \rho_{\ell p}-\frac{1}{2}\left(L_{k}^{\ell i}\right)^{*} L_{k}^{\ell p} \rho_{p j}\right. \\
& \left.-\frac{1}{2}\left(L_{k}^{p \ell}\right)^{*} L_{k}^{p j} \rho_{i \ell}\right] \\
= & -\frac{\mathrm{i}}{\hbar} \sum_{m n} \mathcal{L}_{i j m n} \rho_{m n}+\sum_{m n} \mathcal{D}_{i j m n} \rho_{m n} .
\end{aligned}
$$

Here, $H_{i j}=\langle i|\hat{H}| j\rangle$ and $L_{k}^{i j}=\left\langle i\left|\hat{L}_{k}\right| j\right\rangle$ are the matrix elements of the operators $\hat{H}$ and $\hat{L}_{k}$. Also the superoperators can be represented in form of a matrix, albeit of size $N^{2} \times N^{2}$, with elements

$$
\begin{aligned}
\mathcal{L}_{i j m n}= & H_{i m} \delta_{j n}-H_{n j} \delta_{i m} \\
\mathcal{D}_{i j m n}= & \sum_{k}\left\{L_{k}^{i m}\left(L_{k}^{j n}\right)^{*}\right. \\
& \left.-\frac{1}{2} \sum_{\ell}\left[\left(L_{k}^{\ell i}\right)^{*} L_{k}^{\ell m} \delta_{j n}+\left(L_{k}^{\ell n}\right)^{*} L_{k}^{\ell j} \delta_{i m}\right]\right\}
\end{aligned}
$$

where $\delta$ denotes the Kronecker delta. We emphasize that while Eq. (6) ensures that there is a matrix representation $\mathcal{D}_{i j m n}$ for any given set of Lindblad operators, the converse is not necessarily true, and arbitrarily chosen $\mathcal{D}_{i j m n}$ can produce unphysical results.

\section{B. Choice of Lindblad Operators}

The choice of the $\hat{L}_{k}$ for generating a certain time evolution is not unique. In particular, for a given set $\hat{L}_{k}$ with $k=1, \ldots, K$, the set $\hat{L}_{k}^{\prime}=\sum_{\ell} u_{k \ell} \hat{L}_{\ell}$ (also with $\ell=1, \ldots, K)$ generates the same dynamics for an arbitrary unitary matrix with dimension $K$ and elements $u_{k \ell}{ }^{75 / 85}$ This can easily be verified by substituting the $\hat{L}_{k}$ in Eq. (3) with above expression for $\hat{L}_{k}^{\prime}$, and considering that $\sum_{k} u_{k \ell} u_{k m}^{*}=\delta_{\ell m}$. Furthermore, the $\hat{L}_{k}$ might also contain unitary contributions, which can alternatively be included into the Hamiltonian $\hat{H}$. In particular, replacing an operator $\hat{L}_{k}$ by $\hat{L}_{k}^{\prime}=\hat{L}_{k}+\alpha_{k} \hat{I}$ where $\alpha_{k}$ is an arbitrary complex constant with dimension of inverse square root of time, and $\hat{H}$ by $\hat{H}^{\prime}=\hat{H}+$ $(\mathrm{i} \hbar / 2)\left(\alpha_{k} \hat{L}_{k}^{\dagger}-\alpha_{k}^{*} \hat{L}_{k}\right)$ generates the same dynamics. ${ }^{75} 85$ From the Kraus theorem ${ }^{77}$ it follows that it is always possible to choose the Lindblad operators so that a given non-unitary evolution can be represented by $K \leq N^{2}-1$ operators [in addition to $\hat{L}_{0} \propto \hat{I}$ which gives a vanishing contribution in Eq. (3)]. Formally, such a representation can be constructed by starting from the KossakowskiSudarshan form ${ }^{51}$ of the Lindblad equation and applying a unitary transformation to convert it to Eq. (3) ! However, it has been pointed out that the resulting standard form does not give much insight into the underlying physical processes. ${ }^{[5]}$ From a practical point of view, it is more natural to choose the $\hat{L}_{k}$ so that they represent certain physical effects. In the following, we will discuss the two most relevant mechanisms, i.e., incoherent transitions between states corresponding to hopping transport, and pure dephasing which affects the coherence between two states but does not involve population transfer between them.

\section{Incoherent Transitions}

For a transition from a given basis state $|\alpha\rangle$ to $|\beta\rangle$ with a rate $r_{\alpha \rightarrow \beta}$, the associated Lindblad operator is given by

$$
\hat{L}_{\alpha \rightarrow \beta}=r_{\alpha \rightarrow \beta}^{1 / 2}|\beta\rangle\langle\alpha|,
$$

and Eq. (6) for the corresponding superoperator matrix elements yields

$$
\begin{aligned}
\mathcal{D}_{i j m n}^{\alpha \rightarrow \beta}= & r_{\alpha \rightarrow \beta}\left[\delta_{i \beta} \delta_{j \beta} \delta_{m \alpha} \delta_{n \alpha}\right. \\
& \left.-\frac{1}{2}\left(\delta_{i \alpha} \delta_{j n} \delta_{m \alpha}+\delta_{i m} \delta_{j \alpha} \delta_{n \alpha}\right)\right] .
\end{aligned}
$$

Inserting Eq. (7) into Eq. (4), we obtain population changes $\left[\partial_{t} \rho_{\beta \beta}\right]_{\alpha \rightarrow \beta}=r_{\alpha \rightarrow \beta} \rho_{\alpha \alpha}, \quad\left[\partial_{t} \rho_{\alpha \alpha}\right]_{\alpha \rightarrow \beta}=$ $-r_{\alpha \rightarrow \beta} \rho_{\alpha \alpha}$. The population relaxation is thus generally described by rate equation terms

$$
\left[\partial_{t} \rho_{\alpha \alpha}\right]_{\text {relax }}=\sum_{j \neq \alpha} r_{j \rightarrow \alpha} \rho_{j j}-r_{\alpha} \rho_{\alpha \alpha},
$$


where

$$
r_{\alpha}=\sum_{j \neq \alpha} r_{\alpha \rightarrow j}
$$

is the total outscattering rate from level $\alpha$. Furthermore, we see that apart from the population changes, $\hat{L}_{\alpha \rightarrow \beta}$ also contains the associated lifetime contribution to dephasing, with $\left[\partial_{t} \rho_{\alpha n}\right]_{\alpha \rightarrow \beta}=-\left(r_{\alpha \rightarrow \beta} / 2\right) \rho_{\alpha n}$ and $\left[\partial_{t} \rho_{n \alpha}\right]_{\alpha \rightarrow \beta}=-\left(r_{\alpha \rightarrow \beta} / 2\right) \rho_{n \alpha}$ where $n \neq \alpha$. This means that population transfer from a state $|\alpha\rangle$ to $|\beta\rangle$ induces dephasing not only for this transition, but also for other transitions involving $|\alpha\rangle$, and ignoring this fact might lead to unphysical results. ${ }^{72}$ On the other hand, this implies that the total lifetime contribution to the dephasing rate for a transition $\alpha \rightarrow \beta$ is with Eq. $10 p$ given by $\left(r_{\alpha}+r_{\beta}\right) / 2$, i.e., is obtained from the total outscattering rates for levels $\alpha$ and $\beta$. We note that the operator in Eq. (7) provides an elementary description of transitions, but does for example not take into account correlations between different transition processes.

\section{Pure Dephasing}

In addition to above discussed population changes, there can be additional mechanisms which do not involve population transfer between the chosen basis states, but cause additional decoherence, resulting in a decay of off-diagonal density matrix elements only $[86]$ This socalled pure dephasing contribution between two levels $\alpha$ and $\beta \neq \alpha$ can be described as $\left[\partial_{t} \rho_{\alpha \beta}\right]_{\text {pure }}=-\gamma_{\alpha \beta}^{\prime} \rho_{\alpha \beta}$, which also implies $\left[\partial_{t} \rho_{\beta \alpha}\right]_{\text {pure }}=-\gamma_{\alpha \beta}^{\prime} \rho_{\beta \alpha}$ since $\rho_{\alpha \beta}=$ $\rho_{\beta \alpha}^{*}$. Here, $\gamma_{\alpha \beta}^{\prime}=\gamma_{\beta \alpha}^{\prime} \geq 0$ denotes the pure dephasing rate. As can easily be seen, the corresponding dissipation superoperator in Eq. (4) can be represented by the matrix elements

$$
\mathcal{D}_{i j m n}^{\alpha \beta}=-\gamma_{\alpha \beta}^{\prime}\left(\delta_{i \alpha} \delta_{j \beta} \delta_{m \alpha} \delta_{n \beta}+\delta_{i \beta} \delta_{j \alpha} \delta_{m \beta} \delta_{n \alpha}\right) .
$$

The Lindblad operators for pure dephasing must be diagonal in the chosen basis ${ }^{[85}$ However, Eq. (11) does not generally ensure physical behavior, and thus a representation in terms of Lindblad operators does not always

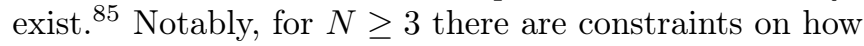
to select the pure dephasing rates $\gamma_{\alpha \beta}^{\prime} \geq 0$ to ensure compatibility with Eq. (3), and an ill-considered choice can for example easily result in a violation of positive semidefiniteness for $\hat{\rho}{ }^{\frac{72185}{185}}$ For example, $\gamma_{12}^{\prime}+\gamma_{13}^{\prime}+\gamma_{23}^{\prime} \leq$ $2\left(\gamma_{12}^{\prime} \gamma_{13}^{\prime}+\gamma_{12}^{\prime} \gamma_{23}^{\prime}+\gamma_{13}^{\prime} \gamma_{23}^{\prime}\right)^{1 / 2}$ must hold in three-level systems, which is already violated if only one of the three pure dephasing rates is non-zero.

In two-level systems, pure dephasing is described by a single rate $\gamma_{12}^{\prime}=\gamma_{21}^{\prime}=\gamma^{\prime} \geq 0$, and can for example be represented by a Lindblad operator $\hat{L}=\left(2 \gamma^{\prime}\right)^{1 / 2}|1\rangle\langle 1|$ or $\hat{L}=\left(2 \gamma^{\prime}\right)^{1 / 2}|2\rangle\langle 2|$, or also by the set $\hat{L}_{1}=\left(\gamma^{\prime}\right)^{1 / 2}|1\rangle\langle 1|$, $\hat{L}_{2}=\left(\gamma^{\prime}\right)^{1 / 2}|2\rangle\langle 2|$. More generally, if the same (typically empirical) pure dephasing rate $\gamma^{\prime}$ is assumed for all transitions of an $N$-level system, $\frac{90191}{1}$ this case can always be represented by Lindblad operators, for example by the set $\hat{L}_{k}=\left(\gamma^{\prime}\right)^{1 / 2}|k\rangle\langle k|, k=1 . . N{ }^{86}$

Taking into account the results of Section IIB 1, the total phase relaxation due to pure dephasing plus lifetime broadening associated with incoherent transitions is described by the dissipation term

$$
\left[\partial_{t} \rho_{\alpha \beta}\right]_{\mathrm{relax}}=-\gamma_{\alpha \beta} \rho_{\alpha \beta}=-\left[\left(r_{\alpha}+r_{\beta}\right) / 2+\gamma_{\alpha \beta}^{\prime}\right] \rho_{\alpha \beta},
$$

where $\gamma_{\alpha \beta}=\left(r_{\alpha}+r_{\beta}\right) / 2+\gamma_{\alpha \beta}^{\prime}$ is the total dephasing rate and the $r_{\alpha, \beta}$ are given by Eq. 100.72

\section{General Case}

While physical dissipation channels can often be represented by either incoherent transitions or pure dephasing, ${ }^{92}$ see Sections II B 1 and II B 2, the Lindblad operators should not a priori be restricted to these two forms, but rather be found based on physical considerations. ${ }^{93.94}$ Even more, the representation of a dissipative channel as, e.g., incoherent transition or pure dephasing, only applies for the chosen basis. ${ }^{85 \mid 90}$ For illustration, let's assume an $N$-level system with orthonormal basis states $|n\rangle$ and dissipative channels described by a set of Lindblad operators $\hat{L}_{k}$. Alternatively, an orthonormal basis with states $\left|n^{\prime}\right\rangle$ can be used, with $|n\rangle=\hat{I}|n\rangle=\sum_{n^{\prime}}\left\langle n^{\prime} \mid n\right\rangle\left|n^{\prime}\right\rangle$, which changes the character of the Lindblad operators in the new basis system. As an illustrative example, we restrict ourselves to two relevant levels $|1\rangle$ and $|2\rangle$, which are assumed to be localized in adjacent potential wells, and between which tunneling through the separating barrier occurs. This mechanism plays for example an important role in QCLs, which are frequently modeled with a density matrix approach for a discrete quantum system, using localized states to describe the tunneling transport across thick barriers 62 $88|89| 95 / 97$ This tunneling process is critically affected by dephasing between the two states involved, which can be modeled by Eq. 12 . $8919195 \mid 98$ We exemplarily focus on the pure dephasing contribution, which can for a two-level system be described by the Lindblad operator $\hat{L}=\left(2 \gamma^{\prime}\right)^{1 / 2}|1\rangle\langle 1|$ as discussed in Section II B 2 Changing to energy eigenstates $\left|1^{\prime}\right\rangle$ and $\left|2^{\prime}\right\rangle$ and for simplicity assuming near-degeneracy, we obtain $|1\rangle=2^{-1 / 2}\left(\left|1^{\prime}\right\rangle+\left|2^{\prime}\right\rangle\right)$ and $|2\rangle=2^{-1 / 2}\left(\left|1^{\prime}\right\rangle-\left|2^{\prime}\right\rangle\right) !^{99} \mathrm{In}$ the energy basis, above Lindblad operator then becomes $\hat{L}=\left(\gamma^{\prime}\right)^{1 / 2}\left(\left|1^{\prime}\right\rangle\left\langle 1^{\prime}|+| 1^{\prime}\right\rangle\left\langle 2^{\prime}|+| 2^{\prime}\right\rangle\left\langle 1^{\prime}|+| 2^{\prime}\right\rangle\left\langle 2^{\prime}\right|\right)$ which is not diagonal, i.e., does not represent pure dephasing in that basis.

To summarize, the frequently used classification of dissipation channels in incoherent transitions and pure dephasing is not always possible and additionally depends on the chosen basis system, but is frequently used since it allows for an intuitive physical interpretation. Thus, this classification might also be helpful for determining the 
corresponding dissipative rates based on compact models or by comparison to experimental data..$^{91100 \mid 101}$ Consequently, for a given system a criterion for a convenient choice of basis states might be that the dissipation channels can reasonably well be described in terms of incoherent transitions and pure dephasing, which for example motivates the frequent use of localized states to describe tunneling transport through thick barriers.

\section{Conditions for Validity}

As discussed in Section IIB, the dissipation parameters must fulfill certain conditions to ensure physical behavior of the density matrix, which is exactly true if a representation of the dissipation process in terms of Lindblad operators exists. For example, the total dephasing rate of a given transition cannot be smaller than the lifetime broadening contribution due to incoherent transitions, as can be seen from Eq. 12. Also, as discussed in Section II B 2, the pure dephasing rates cannot be independently chosen for each transition, but must fulfill certain conditions for $N \geq 3$ levels. Thus, if the experimentally obtained dissipation rates for a system do not satisfy above conditions, this might indicate that the chosen model is not adequate, for example that not enough levels are considered ${ }^{72}$

As noted above, the Lindblad equation can also be microscopically derived for a quantum system weakly coupled to a large Markovian environment ${ }^{75 / 80181}$ These assumptions require in particular that the coherent system dynamics and relaxation processes occur on a slower timescale than the memory decay of the environment $75[81$ These additional microscopic constraints are not required to ensure completely positive and trace preserving evolution of the density matrix, which is guaranteed by the Lindblad form of Eq. (3). However, disregarding the microscopic validity criteria might result in a violation of other laws such as Onsager's relation. ${ }^{94}$ On the other hand, it has been pointed out that some of the assumptions usually invoked in microscopic derivations, such as the secular approximation, might be unnecessarily restrictive ${ }^{94}$ Eventually, for a description of realistic quantum systems where many degrees of freedom affect the time evolution, there will always be a trade-off between exactness and manageability of the model ${ }^{94}$ From a practical point of view, Lindbladtype master equations, such as the MB system, often still yield useful results on the verge of the microscopic validity range, for example in semiconductor structures interacting with high-intensity fields.102,106

\section{OPTICAL BLOCH EQUATIONS}

The most basic quantum system is the two-level system with only $N=2$ relevant states. This can be a natural two-level system with only two eigenstates such as a spin $1 / 2$ particle, or a quasi-two-level system with two strongly coupled states, such as an optical transition in resonance with an electromagnetic field $\underline{49}$ or a driven double-well potential. ${ }^{84}$ In an early application of this model, Rabi investigated the interaction of a spin $1 / 2$ particle with a rotating magnetic field by solving the time dependent Schrödinger equation. ${ }^{14}$ The term "Bloch equations", in the narrow sense, refers to evolution equations for a dissipative two-level system, first devised to describe the evolution of the nuclear magnetic moment in a magnetic field. ${ }^{46}$ Here, the interaction with the environment was taken into account by two phenomenological relaxation time constants. This concept was extended to other two-level systems, such as a pair of levels in resonance with a classical optical field $47 / 48$ The resulting evolution equations are occasionally called optical Bloch equations for distinction. $\frac{49}{}$ The optical propagation can be considered by coupling the Bloch model to Maxwell's equations, ${ }^{24 / 47 / 49}$ resulting in the so-called Maxwell-Bloch (MB) equations. In the following, we focus on the interaction of a quantum system with an optical field, where the coupled MB equations have to be used for a combined description of the system dynamics and optical propagation. Here, we will not restrict ourselves to two-level systems, but rather consider the more general case of $N \geq 2$ discrete levels. The resulting equations are for $N \geq 3$ states occasionally also referred to as a multilevel Bloch/MB model ${ }^{[07}$ Furthermore, for the description of dissipative effects due to the system interaction with the environment, the Lindblad formalism introduced in Section II will serve as a framework. Sometimes the Lindblad equation, Eq. (3), is already referred to as Bloch equations. ${ }^{108}$ In the following, the (optical) Bloch equations will be regarded as a special form of Eq. 3) containing an interaction Hamiltonian $\hat{H}_{1}(t)$ to describe light-matter coupling.

\section{A. Dipole Approximation}

We consider a Hamiltonian of the form $\hat{H}=$ $(\hat{\mathbf{p}}-q \mathbf{A})^{2} /(2 m)+q \varphi+V$, which models the system's interaction with a classical optical field, represented by a time and space dependent magnetic vector potential $\mathbf{A}=\mathbf{A}(\hat{\mathbf{r}}, t)$ and electric potential $\varphi=\varphi(\hat{\mathbf{r}}, t)$. Here, $\hat{\mathbf{r}}$ and $\hat{\mathbf{p}}$ denote the position and (canonical) momentum operators of the quantum system with the commutator $\left[\hat{r}_{i}, \hat{p}_{j}\right]=\mathrm{i} \hbar \delta_{i j}$, which are in position representation given by $\hat{\mathbf{r}}=\mathbf{r}$ and $\hat{\mathbf{p}}=-\mathrm{i} \hbar \nabla$, and $V=V(\hat{\mathbf{r}})$ represents the system's potential energy. Furthermore, $m$ and $q$ denote the carrier mass and charge, which are for electrons given by $m=m_{\mathrm{e}}$ and $q=-e$, with the elementary charge $e$. Using the Coulomb gauge $\nabla \mathbf{A}=0$, we have $[\mathbf{A}, \mathbf{p}]=0$. Furthermore assuming a radiation field without free charge contributions gives $\varphi=0$, and $\mathbf{E}=-\partial_{t} \mathbf{A}$ for the corresponding electric field 109 Under these assumptions, we obtain $\hat{H}=\hat{H}_{0}+\hat{H}_{1}$ with the Hamiltonian of the unperturbed system $\hat{H}_{0}=\hat{\mathbf{p}}^{2} /(2 m)+V$, and the 
time dependent interaction Hamiltonian 110

$$
\hat{H}_{1}=-(q / m) \mathbf{A} \hat{\mathbf{p}}+q^{2} \mathbf{A}^{2} /(2 m) .
$$

The Bloch equations are then obtained from Eq. (4) by choosing the energy eigenstates of the system Hamiltonian $\hat{H}_{0}$ as basis, resulting in matrix elements $H_{0, i j}=$ $E_{i} \delta_{i j}$ where $E_{i}$ is the eigenenergy of state $i$, and

$$
H_{1, i j}=-\frac{q}{m}\langle i|\mathbf{A} \hat{\mathbf{p}}| j\rangle+\frac{q^{2}}{2 m}\left\langle i\left|\mathbf{A}^{2}\right| j\right\rangle .
$$

Typically, the field varies on the scale of the optical wavelengths involved, and the system dimensions are much smaller. The carriers do then not experience a spatial field variation across the quantum system, and $\mathbf{A}$ in Eqs. (13) and (14) can be represented by a space independent vector potential, evaluated at the macroscopic position of the quantum system. In this case, it can be shown by a gauge transformation that the interaction Hamiltonian in Eq. (13) is equivalent to

$$
\hat{H}_{1}=-\hat{\mathbf{d}} \mathbf{E}(t),
$$

which corresponds to the interaction Hamiltonian in the widely used (electric) dipole approximation! 109/110 Here, $\hat{\mathbf{d}}=q \hat{\mathbf{r}}$ denotes the system's dipole operator, and the electric field $\mathbf{E}(t)$ is taken at the system position. Intuitively, the Hamiltonian in Eq. 15 corresponds to the potential energy associated with the force $q \mathbf{E}(t)$ exerted by the electric field on the carriers.

We note that under some special conditions, such as high harmonic generation 111 or strong plasmonic confinement in nanophotonic structures, $\frac{112113}{12}$ the field gradient may become so large that the dipole approximation is not applicable. In this context, we re-emphasize that Eq. 15 only assumes a spatially constant field within a given quantum system, but does not neglect the term $\propto \mathbf{A}^{2}$ in Eq. 13 and is thus not restricted to weak fields, as is sometimes believed. For the interaction Hamiltonian in Eq. 15, the mechanical and canonical momentum operators coincide, $m \hat{\mathbf{v}}=\hat{\mathbf{p}}$. The Hamiltonian of the unperturbed system $\hat{H}_{0}=\hat{\mathbf{p}}^{2} /(2 m)+V$ thus corresponds to the instantaneous energy operator, and a matrix element $\rho_{i i}$ in the eigenstate basis $|i\rangle$ of $H_{0}$ can be interpreted as the measurable probability of finding the system in the corresponding energy eigenstate ${ }^{[10}$ For the interaction Hamiltonian in Eq. 13, the mechanical momentum operator is $m \hat{\mathbf{v}}=\hat{\mathbf{p}}-q \mathbf{A}$. This complicates the physical interpretation of results, since, e.g., the instantaneous energy operator $m \hat{\mathbf{v}}^{2} / 2+V$ is different from $\hat{H}_{0}$, which prohibits an interpretation of $\rho_{i i}$ as a measurable probability $110|114| 115$ These differences also explain why the matrix elements $H_{1, i j}$ in Eq. (14) for spatially constant $\mathbf{A}$ and those obtained from Eq. (15) deviate from each other 110 While both versions of the interaction Hamiltonian lead to identical results for observable quantities as expected, it has been pointed out that the use of approximations, such as the rotating wave approximation discussed in Section IIIE can cause deviations between the two formulations $114[115$ In the following, we will use the interaction operator of the form Eq. (15).

\section{B. Optical Bloch Equations in Standard Form}

From Eq. 4), we obtain with Eq. 15 in the dipole approximation the (multilevel) Bloch equations

$$
\begin{aligned}
\partial_{t} \rho_{i j}= & -i \omega_{i j} \rho_{i j}+\frac{\mathrm{i}}{\hbar} \sum_{\ell}\left(\mathbf{d}_{i \ell} \rho_{\ell j}-\mathbf{d}_{\ell j} \rho_{i \ell}\right) \mathbf{E} \\
& +\sum_{m n} \mathcal{D}_{i j m n} \rho_{m n}
\end{aligned}
$$

with the transition frequencies $\omega_{i j}=\left(E_{i}-E_{j}\right) / \hbar$. If we furthermore restrict the description of dissipative effects to incoherent transitions and dephasing, Eqs. (9) and 12 , Eq. 16) simplifies to

$$
\begin{aligned}
\partial_{t} \rho_{i j}= & -\mathrm{i} \omega_{i j} \rho_{i j}+\frac{\mathrm{i}}{\hbar} \sum_{\ell}\left(\mathbf{d}_{i \ell} \rho_{\ell j}-\mathbf{d}_{\ell j} \rho_{i \ell}\right) \mathbf{E} \\
& -\gamma_{i j} \rho_{i j}, i \neq j \\
\partial_{t} \rho_{i i}= & \frac{\mathrm{i}}{\hbar} \sum_{\ell}\left(\mathbf{d}_{i \ell} \rho_{\ell i}-\mathbf{d}_{\ell i} \rho_{i \ell}\right) \mathbf{E}+\sum_{j \neq i} r_{j \rightarrow i} \rho_{j j}-r_{i} \rho_{i i}
\end{aligned}
$$

Although quantum optoelectronic devices can in principle comprise a single isolated quantum system, for example a QD, 116/117 in general they are based on extended nanostructures such as quantum well structures, or an ensemble of many quantum systems such as multiquantum-dot structures. This requires a position resolved model, where the device is described by a representative quantum system with density matrix $\rho_{i j}(\mathbf{x}, t)$ at each device position $\mathbf{x}$. Furthermore, also the parameters $\omega_{i j}, \mathbf{d}_{i j}, \gamma_{i j}, r_{j \rightarrow i}$ and $r_{i}$ in Eqs. (16) and (17) generally depend on $\mathbf{x}$ for inhomogeneous device structures, 118|119 such as multi-section lasers 120,122

\section{Optical Dipole Matrix Element}

The Hamiltonian part of the Bloch equations, Eqs. 16 and (17), requires the dipole matrix element vectors $\mathbf{d}_{i \ell}$ of the optical transitions and the eigenenergies of the quantized states as an input. These can be computed from models derived from the stationary Schrödinger equation, such as the effective mass or k.p approach, as shortly discussed in the following.

In Fig. 3, the band structure of GaAs as an exemplary direct bandgap semiconductor material is displayed. Shown is the conduction band (solid line) and the valence band (dashed lines), consisting of heavy hole, light hole and split-off band. The holes tend to accumulate near the valence band maximum which is always at the $\Gamma$ point where the crystal wavevector is $\mathbf{k}=\mathbf{0}$. 


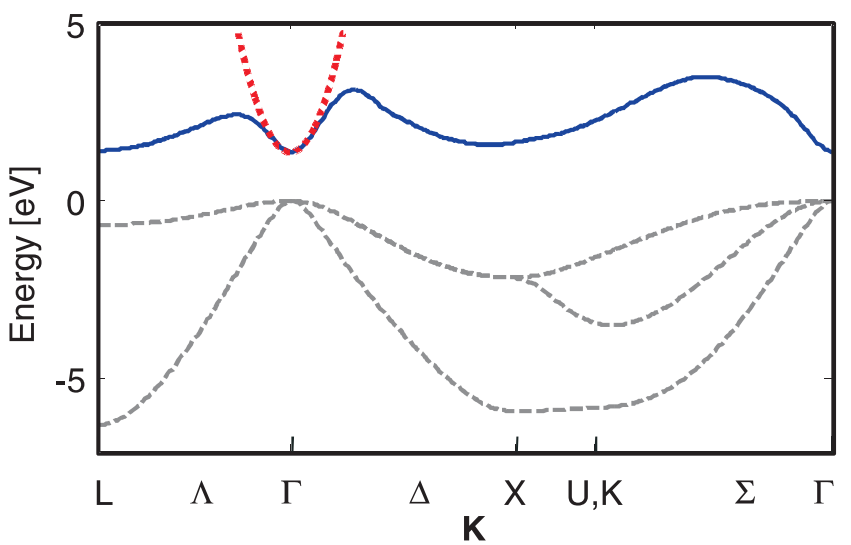

FIG. 3. Band structure of gallium arsenide (GaAs), obtained based on a simple pseudo-potential tight-binding method without spin-orbit coupling 123 Shown is the valence band (dashed lines), the conduction band (solid line) and the parabolic dispersion relation assumed for the $\Gamma$ valley in effective mass approximation (dotted line).

For direct bandgap semiconductors, the global conduction band minimum where the electrons accumulate happens to be also at the $\Gamma$ point, and thus conservation of crystal momentum can be satisfied for radiative electronhole recombination. This process is much less likely in indirect bandgap semiconductors, where the global conduction band minimum is not at the $\Gamma$ point and the process must additionally involve a phonon or crystal defect to achieve momentum conservation.

Assuming a direct bandgap semiconductor, it is practical to write the full wavefunction $F_{i}(\mathbf{r})$ of the initial and final state as a product of periodic Bloch function $u_{v_{i}}(\mathbf{r})$ at the $\Gamma$ point of band $v_{i}$ and an envelope wavefunction $\varphi_{i}(\mathbf{r})$ describing the slowly varying spatial modulation of the full wavefunction across the nanostructure $\frac{124}{}$ While a quantized state in a given band generally also contains contributions from neighboring bands, in a first approximation only the contribution of the dominant band is considered,

$$
F_{i}(\mathbf{r})=u_{v_{i}}(\mathbf{r}) \varphi_{i}(\mathbf{r}) .
$$

In quantum well structures, the material composition changes only along the growth direction $x$. Here, quantum confinement only occurs in $x$ direction, while the carriers can move freely in the $y z$-plane. Thus, we can make the ansatz

$$
\varphi_{i}(\mathbf{r})=S^{-1 / 2} \psi_{i}(x) \exp \left(\mathrm{i} k_{y} y+\mathrm{i} k_{z} z\right) .
$$

Here, $S$ is the in-plane cross section area, $\mathbf{k}=\left[k_{y}, k_{z}\right]^{\mathrm{T}}$ denotes the in-plane wavevector in the $y z$-plane where $\mathrm{T}$ indicates the transpose, and $\psi_{i}(x)$ is the (generally $\mathbf{k}$ dependent) one-dimensional envelope wavefunction in confinement direction. In Fig. 4, the full wavefunctions $F_{i}$ and corresponding envelope wavefunctions $\varphi_{i}$ are schematically illustrated for the two lowest conduction band states and the valence band ground state

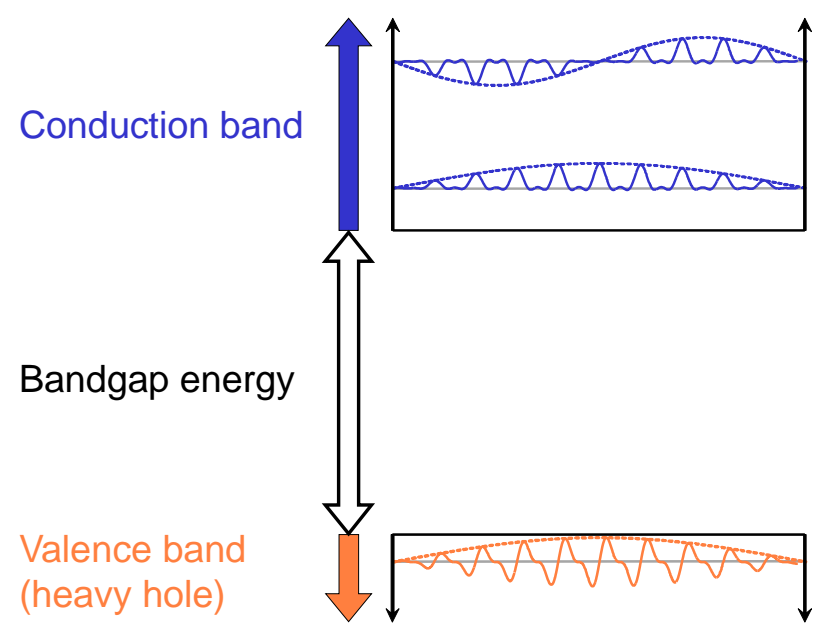

FIG. 4. Schematic illustration of full bound state wavefunctions $F_{i}=u_{v_{i}} \varphi_{i}$ (solid) and envelope wavefunctions $\varphi_{i}$ (dashed) in the conduction and valence band of a quantum well.

of a quantum well. Similar considerations apply to quantum wires, where quantum confinement occurs in two dimensions while the carriers can move freely along the third coordinate. In QDs, the carriers are confined in all three dimensions.

\section{Computation of Envelope Wavefunction}

Neglecting the coupling between conduction and valence bands, the simplest model for computing $\varphi_{i}(\mathbf{r})$ in a quantum structure is the Ben Daniel-Duke model, which works well for low-lying conduction band states in the $\Gamma$ valley and also generally at the heavy hole valence band maximum 124 Here, we describe the dispersion relation between energy and wavevector around the $\Gamma$ point by $E(\mathbf{k})=V+\hbar^{2}|\mathbf{k}|^{2} /\left(2 m^{*}\right)$ which corresponds to a second order expansion, as illustrated by the dotted line in Fig.3. The position dependent material composition in nanostructures causes the effective mass $m^{*}$ and band edge energy $V$ to depend on $\mathbf{r}$, where $V$ additionally contains the externally applied bias. Within this model, the stationary effective mass Schrödinger equation is given by 124

$$
0=\left\{-\frac{\hbar^{2}}{2}\left[\nabla \frac{1}{m^{*}(\mathbf{r})} \nabla\right]+V(\mathbf{r})-E_{i}\right\} \varphi_{i}(\mathbf{r}),
$$

where $E_{i}$ denotes the eigenenergy of state $i$. For the valence band, commonly the hole picture is adopted to avoid a negative effective mass in Eq. 20). For the transition between a conduction band electron state with eigenenergy $E_{i}$ and a valence band hole state with energy $E_{j}$, the transition energy is then given by $E_{i}+E_{j}+E_{\mathrm{g}}$ where $E_{\mathrm{g}}$ denotes the bandgap energy, i.e., the energy difference between valence band maximum and conduction band minimum. In quantum well systems, $m^{*}$ and 
$V$ only depend on the $x$ coordinate, and Eq. 20 can be reduced to the one-dimensional effective mass equation by inserting Eq. (19). Similar considerations apply to quantum wires where $m^{*}$ and $V$ only depend on two coordinates.

The Ben Daniel-Duke model in Eq. 20 can be extended, e.g., by accounting for band bending due to space charge effects in the potential, which is self-consistently included by solving Eq. 20 together with the Poisson equation. ${ }^{125 \mid 126]}$ Furthermore, an energy dependent effective mass can be introduced to include nonparabolicity effects associated with the deviation of the dispersion relation from the parabolic form assumed above.127/128

A further refined treatment of the conduction and valence bands, which accounts for band coupling, is usually performed based on k.p theory, initially proposed by Kane ${ }^{129} 130$ and Luttinger and Kohn!131 Here the envelope wavefunctions are not scalar, but a multicomponent vector containing contributions from all the bands considered. In many structures, strain arising from the lattice mismatch between the different semiconductor compounds plays an important role, and can be considered based on the Bir-Pikus model ${ }^{[132}$ For modeling interband devices, eight-band k.p is a common option which considers the top three valence bands and the lowest conduction band, along with spin orientation.124|133 This approach is routinely applied to nanostructures, such as quantum dots, ${ }^{134}$ wires ${ }^{135}$ and wells ${ }^{136}$ If only valence band states are considered, a restriction to six bands is possible ${ }^{137}$ This approach is sometimes also combined with Eq. 20 for the conduction band, assuming that it is decoupled from the valence bands. On the other hand, it has been found that for certain cases, eight-band k.p is not accurate enough. For example, a 14-band k.p approach which also includes the second conduction band in III$\mathrm{V}$ semiconductors has been developed to obtain a more accurate conduction band dispersion relation at higher energies, 138 and 14 -band k.p has also yielded improved results for $\mathrm{SiGe} / \mathrm{Si}$ heterostructures. 139

\section{Inter- and Intraband Dipole Matrix Elements}

The dipole matrix element is best evaluated by computing the expectation value of the momentum operator $\hat{\mathbf{p}}=-\mathrm{i} \hbar \nabla$. Employing the product rule and exploiting the fact that the periodic Bloch functions and envelope wavefunctions vary on two different length scales, we can with Eq. 18 write 140

$$
\left\langle F_{i}|\hat{\mathbf{p}}| F_{j}\right\rangle \approx\left\langle\varphi_{i} \mid \varphi_{j}\right\rangle\left\langle u_{v_{i}}|\hat{\mathbf{p}}| u_{v_{j}}\right\rangle+\left\langle u_{v_{i}} \mid u_{v_{j}}\right\rangle\left\langle\varphi_{i}|\hat{\mathbf{p}}| \varphi_{j}\right\rangle .
$$

For transitions between conduction and valence band states, the first term dominates because the Bloch functions vary much more rapidly than the envelope wavefunctions. Using $\left\langle F_{i}|\hat{\mathbf{p}}| F_{j}\right\rangle=\mathrm{i} m_{\mathrm{e}} E_{\mathrm{g}}\left\langle F_{i}|\hat{\mathbf{r}}| F_{j}\right\rangle / \hbar$ with the electron mass $m_{\mathrm{e}}$ and band gap energy $E_{\mathrm{g}}, 141$ the interband dipole matrix element can then in a first ap- proximation be written as

$$
\left\langle F_{i}|\hat{\mathbf{r}}| F_{j}\right\rangle=-\mathrm{i} \hbar m_{\mathrm{e}}^{-1} E_{\mathrm{g}}^{-1}\left\langle u_{v_{i}}|\hat{\mathbf{p}}| u_{v_{j}}\right\rangle\left\langle\varphi_{i} \mid \varphi_{j}\right\rangle .
$$

For intraband optical transitions, we have $v_{i}=v_{j}$, $\left\langle u_{v_{i}}|\hat{\mathbf{p}}| u_{v_{i}}\right\rangle=0$ and $\left\langle u_{v_{i}} \mid u_{v_{i}}\right\rangle=1$, and thus Eq. 21] yields $\left\langle F_{i}|\hat{\mathbf{p}}| F_{j}\right\rangle \approx\left\langle\varphi_{i}|\hat{\mathbf{p}}| \varphi_{j}\right\rangle$ and analogously

$$
\left\langle F_{i}|\hat{\mathbf{d}}| F_{j}\right\rangle \approx\left\langle\varphi_{i}|\hat{\mathbf{d}}| \varphi_{j}\right\rangle
$$

In quantum well systems, confinement only occurs in the growth direction $x$, and the envelope wavefunction has the form given by Eq. 19 . For transitions between a conduction band state $\left|\psi_{i}, \mathbf{k}\right\rangle$ and a valence band state $\left|\psi_{j}, \mathbf{k}^{\prime}\right\rangle,\left\langle\varphi_{i} \mid \varphi_{j}\right\rangle=\left\langle\psi_{i} \mid \psi_{j}\right\rangle \delta_{\mathbf{k}, \mathbf{k}^{\prime}}$, i.e., the optical transition is $\mathbf{k}$ conserving. The absolute value of the dipole matrix element can be approximately written as

$$
\left|\mathbf{e}\left\langle F_{i \mathbf{k}}|\hat{\mathbf{r}}| F_{j \mathbf{k}^{\prime}}\right\rangle\right|=c_{i j} E_{\mathrm{g}}^{-1} P_{\mathrm{cv}}\left\langle\psi_{i} \mid \psi_{j}\right\rangle \delta_{\mathbf{k}, \mathbf{k}^{\prime}}
$$

where e denotes the polarization direction of the electric field, and $P_{\mathrm{cv}} \approx 0.85 . .1 \mathrm{~nm} \times \mathrm{eV}$ for most common semiconductors!140 For transitions between conduction band and heavy hole states, $c_{i j}=2^{-1 / 2}$ for polarization in in-plane direction and $c_{i j}=0$ for polarization in growth direction. For transitions between conduction band and light hole states, $c_{i j}=6^{-1 / 2}$ for polarization in in-plane direction and $c_{i j}=2 \times 6^{-1 / 2}$ for polarization in growth direction. For intraband transitions occurring between quantized levels in the conduction band of quantum wells, as are for example employed for QCLs, the envelope wavefunctions again assume the form Eq. 19. The dipole matrix element between an initial state $\left|\psi_{i}, \mathbf{k}\right\rangle$ and a final state $\left|\psi_{j}, \mathbf{k}^{\prime}\right\rangle$ is then with Eq. 23) given by $\mathbf{d}_{i \mathbf{k}, j \mathbf{k}^{\prime}}=\mathbf{d}_{i j} \delta_{\mathbf{k}, \mathbf{k}^{\prime}}$, where

$$
\mathbf{d}_{i j}=\left\langle\psi_{i}|\hat{\mathbf{d}}| \psi_{j}\right\rangle=-e \mathbf{e}_{x} \int \psi_{i}^{*} x \psi_{j} \mathrm{~d} x .
$$

Here, $\mathbf{e}_{x}$ denotes the unit vector in $x$ direction, and only the dipole matrix element for polarization in growth direction $x$ is nonzero. Notably, this is different from transitions between conduction band and heavy hole states in quantum wells where the $x$ component of $\mathbf{d}_{i j}$ is zero, as discussed above. In Fig.5 the possible field polarization directions for interband [Fig. [5(a), (b)] and intraband [Fig. [5(c)] transitions are indicated. For quantum well lasers, Fig.5(a), (b) and (c) correspond to the standard edge-emitting, vertical-cavity surface-emitting and quantum cascade laser.

In quantum dots, the uppermost valence band eigenstates usually exhibit heavy hole character.142 145 Thus, band coupling effects can often be neglected in Eq. 22 for interband transitions between the heavy-hole-like states and low-lying conduction band states. Within the framework of these assumptions, only optical dipole transitions between hole and electron states with equal quantum numbers are allowed, and the envelope wavefunction overlap in Eq. 22 typically approaches $\left\langle\varphi_{i} \mid \varphi_{j}\right\rangle \approx 1$ for 

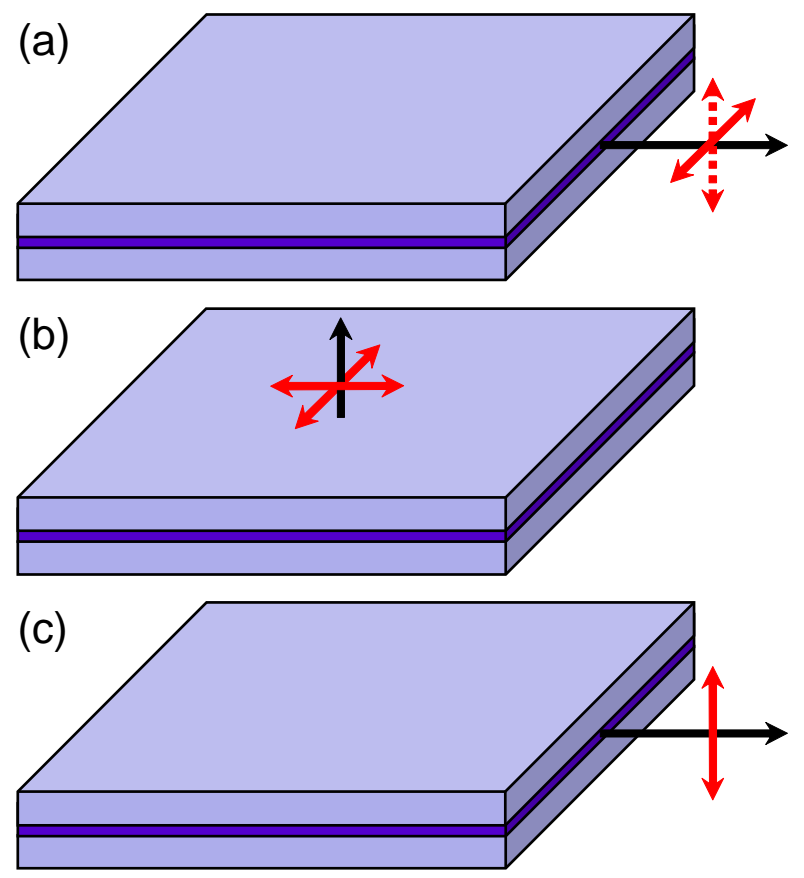

FIG. 5. Polarizations with non-vanishing dipole matrix elements for (a) and (b) interband and (c) intraband transitions. The dashed arrow in (a) indicates that for this polarization, only transitions between conduction band and light hole states contribute.

the allowed transitions $\frac{146 \mid 147}{1}$ The symmetry of the wavefunctions can however be affected by inhomogeneities in shape and composition of the quantum dots as well as piezoelectric fields, resulting in additional weakly allowed transitions. $\frac{148 \mid 149}{}$ Moreover, due to the strong confinement in quantum dots, Coulomb interactions tend to play a pronounced role, causing energy shifts as well as somewhat altered selection rules. Such effects can be included in a more complete description based on the electron-hole-pair picture, which replaces the singlecarrier envelope wavefunctions $\varphi_{i}$ and $\varphi_{j}$ in Eq. 22 by expressions for the excited electron-hole pair state and the corresponding ground state ${ }^{[146 \mid 148}$ Intraband transitions, which are mainly relevant in the context of quantum dot infrared photodetectors, are again described by Eq. 23).

\section{Non-Redundant Density Matrix Representation}

For a discrete-level system with $N$ eigenstates $|j\rangle$, the density matrix contains $N$ real diagonal elements and $N^{2}-N$ complex off-diagonal elements which are related by $\rho_{i j}=\rho_{j i}^{*}$. Furthermore considering the trace condition $\operatorname{Tr}\{\hat{\rho}\}=1$, the density matrix can be represented by $N^{2}-1$ non-redundant, real-valued elements, which are conveniently written as a vector $\mathbf{S}$. This non-redundant representation is for example achieved by the coherence vector (or pseudospin) representation, $\frac{150}{}$ which has also been found useful for numerically efficient implementations of the MB equations. $\frac{[53 \mid 151-153}{150}$ For this purpose, the density matrix operator $\hat{\rho}$ is composed as

$$
\hat{\rho}=\frac{1}{N} \hat{I}+\frac{1}{2} \sum_{j=1}^{N^{2}-1} S_{j} \hat{s}_{j}
$$

Here, $\hat{s}_{j}$ are generators of the Lie algebra of $\mathrm{SU}(N)$ which are traceless Hermitian operators fulfilling the condition $\operatorname{Tr}\left\{\hat{s}_{j} \hat{s}_{k}\right\}=2 \delta_{j k}$, and $\hat{I}$ is the identity operator. $\hat{I}$ and $\hat{s}_{j}$ can be represented by corresponding $N \times N$ matrices. A possible choice for the generators $\hat{s}=\left\{\hat{u}_{12}, \hat{u}_{13}, \ldots, \hat{v}_{12}, \ldots, \hat{w}_{1}, \ldots, \hat{w}_{N-1}\right\}$ consists of $N(N-1) / 2$ generator pairs

$$
\begin{aligned}
& \hat{u}_{j k}=\hat{t}_{j k}+\hat{t}_{k j}, \\
& \hat{v}_{j k}=-\mathrm{i}\left(\hat{t}_{j k}-\hat{t}_{k j}\right),
\end{aligned}
$$

and $N-1$ generators

$$
\hat{w}_{l}=-\left[2 l^{-1}(l+1)^{-1}\right]^{1 / 2}\left(\sum_{\ell=1}^{l} \hat{t}_{\ell \ell}-l \hat{t}_{l+1, l+1}\right),
$$

where $\hat{t}_{j k}=|j\rangle\langle k|$ is the transition-projection operator, and the indices satisfy $1 \leq j<k \leq N$ and $1 \leq l \leq$ $N-1 \sqrt{1500}$ For $N=2$ and $N=3$ these generators produce the Pauli and the Gell-Mann matrices, respectively.

The elements of the coherence vector $\mathbf{S}$ are defined as $S_{j}=\operatorname{Tr}\left\{\hat{\rho} \hat{s}_{j}\right\}$ using the Hilbert-Schmidt inner product. Since both $\hat{\rho}$ and the generators $\hat{s}_{j}$ are Hermitian, the vector elements are real. A similar transform can be applied to the Lindblad equation. Inserting Eq. (26) into Eq. (3) and applying $\operatorname{Tr}\left\{\cdot \hat{s}_{k}\right\}$ yields

$$
\begin{aligned}
\operatorname{Tr}\left\{\partial_{t} \hat{\rho} \hat{s}_{k}\right\} & =\operatorname{Tr}\left\{\frac{1}{2} \sum_{j=1}^{N^{2}-1} \partial_{t} S_{j} \hat{s}_{j} \hat{s}_{k}\right\} \\
& =\frac{1}{2} \sum_{j=1}^{N^{2}-1} \partial_{t} S_{j} \operatorname{Tr}\left\{\hat{s}_{j} \hat{s}_{k}\right\}=\partial_{t} S_{k}
\end{aligned}
$$

for the left hand side. For the right hand side we can write

$\operatorname{Tr}\left\{\mathcal{L}(\hat{\rho}) \hat{s}_{k}+\mathcal{D}(\hat{\rho}) \hat{s}_{k}\right\}=\operatorname{Tr}\left\{\mathcal{L}(\hat{\rho}) \hat{s}_{k}\right\}+\operatorname{Tr}\left\{\mathcal{D}(\hat{\rho}) \hat{s}_{k}\right\}$,

$\operatorname{Tr}\left\{\mathcal{L}(\hat{\rho}) \hat{s}_{k}\right\}=\underbrace{\operatorname{Tr}\left\{N^{-1} \mathcal{L}(\hat{I}) \hat{s}_{k}\right\}}_{=0}+\sum_{j=1}^{N^{2}-1} \underbrace{\frac{1}{2} \operatorname{Tr}\left\{\mathcal{L}\left(\hat{s}_{j}\right) \hat{s}_{k}\right\}}_{=L_{j k}} S_{j}$,

$\operatorname{Tr}\left\{\mathcal{D}(\hat{\rho}) \hat{s}_{k}\right\}=\underbrace{\operatorname{Tr}\left\{N^{-1} \mathcal{D}(\hat{I}) \hat{s}_{k}\right\}}_{S_{j}^{\text {eq }}}+\sum_{j=1}^{N^{2}-1} \underbrace{\frac{1}{2} \operatorname{Tr}\left\{\mathcal{D}\left(\hat{s}_{j}\right) \hat{s}_{k}\right\}}_{D_{j k}} S_{j}$, 
since both superoperators $\mathcal{L}$ and $\mathcal{D}$ are linear. Noting that $\mathcal{L}(\hat{I})=0$ and arranging Eqs. 29 and $30 \mathrm{a}-30 \mathrm{c}$ in matrix-vector form yields

$$
\partial_{t} \mathbf{S}=(L+D) \mathbf{S}+\mathbf{S}^{\mathrm{eq}}
$$

where $L$ and $D$ are $\left(N^{2}-1\right) \times\left(N^{2}-1\right)$ real matrices and $\mathbf{S}^{\mathrm{eq}}$ denotes the equilibrium coherence vector.

Alternatively, one can start from Eq. 44, where the superoperators $\mathcal{L}$ and $\mathcal{D}$ are represented as $N^{2} \times N^{2}$ matrices. This Liouville space representation was used for example in $\frac{154}{1}$ where column-major order was applied to map the indices $(i, j) \mapsto k$ and $(m, n) \mapsto l$. In this case, the density matrix is represented as $N^{2}$ column vector $\mathbf{R}$, and the Lindblad equation reads 154

$$
\partial_{t} \mathbf{R}=\left(-\frac{\mathrm{i}}{\hbar} \mathcal{L}+\mathcal{D}\right) \mathbf{R}
$$

where $\mathcal{L}=\hat{H}^{\mathrm{T}} \otimes \hat{I}-\hat{I} \otimes \hat{H}$ and

$$
\mathcal{D}=\sum_{k}\left[\hat{L}_{k}^{\mathrm{T}} \otimes \hat{L}_{k}-\frac{1}{2}\left(\hat{L}_{k}^{\mathrm{T}} \hat{L}_{k}^{*} \otimes \hat{I}+\hat{I} \otimes \hat{L}_{k}^{\dagger} \hat{L}_{k}\right)\right] .
$$

Here, $\otimes$ denotes the Kronecker product. Then, since the Hilbert-Schmidt inner product reads $\operatorname{Tr}\left\{\hat{a}^{\dagger} \hat{b}\right\}=\mathbf{a}^{\dagger} \mathbf{b}=$ $\mathbf{b}^{\dagger} \mathbf{a}$ in this representation, where the vectors $\mathbf{a}$ and $\mathbf{b}$ are the matrices $\hat{a}$ and $\hat{b}$ in column-major order, we can write the transform from Liouville space to the coherence vector representation as

$$
S_{j}=\operatorname{Tr}\left\{\hat{\rho} \hat{s}_{j}\right\}=\mathbf{s}_{j}^{\dagger} \mathbf{R}, \quad \mathbf{S}=T^{\dagger} \mathbf{R},
$$

where the columns of the transformation matrix $T$ are the generators $\mathbf{s}_{j}$ in column-major order. Conversely, the vector $\mathbf{R}$ can be recovered by

$$
\mathbf{R}=\frac{1}{N} \mathbf{I}+\frac{1}{2} T \mathbf{S}
$$

where $\mathbf{I}$ is the identity matrix in vectorized form. Using these transform relations, we can rewrite Eq. (32) as

$$
\frac{1}{2} T \partial_{t} \mathbf{S}=\left(-\frac{\mathrm{i}}{\hbar} \mathcal{L}+\mathcal{D}\right) \frac{1}{2} T \mathbf{S}+\left(-\frac{\mathrm{i}}{\hbar} \mathcal{L}+\mathcal{D}\right) \frac{1}{N} \mathbf{I}
$$

and simplify the result by left-multiplication with $T^{\dagger}$ to

$$
\partial_{t} \mathbf{S}=\left(-\frac{\mathrm{i}}{2 \hbar} T^{\dagger} \mathcal{L} T+\frac{1}{2} T^{\dagger} \mathcal{D} T\right) \mathbf{S}+\frac{1}{N} T^{\dagger} \mathcal{D} \mathbf{I},
$$

where we used the orthogonality of the generators $\left(\frac{1}{2} T^{\dagger} T=I\right.$, where $I$ is the $N^{2} \times N^{2}$ identity matrix) and the fact that the commutator of the identity is zero $(\mathcal{L} \mathbf{I}=0)$. This corresponds to Eq. (31).

As we shall see in Section IV the derivative of the macroscopic polarization $\partial_{t} \mathbf{P}_{\mathrm{q}}$ has to be calculated for the Maxwell-Bloch equations. Naturally, it must be expressed as function of the vector $\mathbf{S}$. By replacing the trace operation and inserting the transformation rule, we can write for this term

$$
\begin{aligned}
\partial_{t} \mathbf{P}_{\mathrm{q}} & =n_{3 \mathrm{D}} \operatorname{Tr}\left\{\partial_{t} \hat{\rho} \hat{\mathbf{d}}\right\}=n_{3 \mathrm{D}} \mathbf{u}^{\dagger} \partial_{t} \mathbf{R} \\
& =\frac{1}{2} n_{3 \mathrm{D}} \mathbf{u}^{\dagger} T\left[(L+D) \mathbf{S}+\mathbf{S}^{\mathrm{eq}}\right],
\end{aligned}
$$

where $\mathbf{u}$ is the vectorized dipole moment operator and $n_{3 \mathrm{D}}$ denotes the carrier number density. Note that the elements of $\mathbf{u}$ could be vectors themselves, depending on whether one ore more dimensions are considered.

Using the dipole approximation, Eq. (15), we plug in the Hamiltonian $\hat{H}=\hat{H}_{0}-\hat{\mathbf{d}} \mathbf{E}(t)$, which can be represented with two matrices $\mathcal{L}_{0}$ and $\mathcal{L}_{1}$ in Liouville space (and two matrices $L_{0}$ and $L_{1}$ in coherence vector representation, respectively). Since

$$
\begin{aligned}
\mathbf{u}^{\dagger} \mathcal{L}_{1} & =-\mathbf{u}^{\dagger}\left[\hat{\mathbf{d}}^{\mathrm{T}} \mathbf{E}(t) \otimes \hat{I}-\hat{I} \otimes \hat{\mathbf{d}} \mathbf{E}(t)\right] \\
& =-\left[\hat{\mathbf{d}}^{\mathrm{T}} \mathbf{E}(t) \otimes \hat{I} \mathbf{u}-\hat{I} \otimes \hat{\mathbf{d}} \mathbf{E}(t) \mathbf{u}\right]^{\dagger} \\
& =-[\operatorname{vec}(\hat{I} \hat{\mathbf{d}} \hat{\mathbf{d}} \mathbf{E}(t))-\operatorname{vec}(\hat{\mathbf{d}} \mathbf{E}(t) \hat{\mathbf{d}} \hat{I})]^{\dagger}=0
\end{aligned}
$$

where vec denotes the vectorization of an operator, $\operatorname{vec}(\hat{\mathbf{d}})=\mathbf{u}$, and the Hermitian property of the operators involved as well as the properties of the Kronecker product have been exploited, the polarization does not depend on the electric field and Eq. 38 can be refined as

$$
\partial_{t} \mathbf{P}_{\mathrm{q}}=\frac{1}{2} n_{3 \mathrm{D}} \mathbf{u}^{\dagger} T\left[\left(L_{0}+D\right) \mathbf{S}+\mathbf{S}^{\mathrm{eq}}\right]
$$

\section{E. Rotating Wave Approximation}

The Bloch equations (17) are solvable only under special conditions, like $|\Delta M|=1$ transitions in hydrogenlike atoms excited with circularly polarized light!14/49] In particular, closed analytical solutions do not exist for the basic and very important case of excitation with a monochromatic, linearly polarized field ${ }^{155}$ Furthermore, the numerical solution of the Maxwell-Bloch equations requires high spatiotemporal resolution since the fields as well as the off-diagonal density matrix elements in Eq. (17) oscillate with the optical period. For these reasons, the rotating wave approximation (RWA) is commonly invoked, which significantly reduces the numerical burden and enables an analytical treatment of the Bloch equations, at least for incident monochromatic radiation and some other relevant cases.

The RWA is only applicable for not too broadband optical fields, which can then be separated into a slowly varying amplitude, given in complex notation by $\underline{\mathbf{E}}(\mathbf{x}, t)=|\underline{\mathbf{E}}(\mathbf{x}, t)| \exp [\mathrm{i} \phi(\mathbf{x}, t)]$, and a rapidly oscillating carrier with frequency $\omega_{c}>0$. We note that there is no unique definition of $\omega_{\mathrm{c}}$, but rather any choice which 
ensures that all relevant spectral components are close to $\omega_{\mathrm{c}}$ will suffice (for optical fields with symmetric power spectra, it obviously makes sense to pick the center frequency). In complex notation, the electric field can then be written as

$$
\mathbf{E}=\frac{1}{2} \underline{\mathbf{E}} \exp \left(-\mathrm{i} \omega_{\mathrm{c}} t\right)+c . c .,
$$

where c.c. denotes the complex conjugate. Furthermore assuming that all transitions between pairs of states $i$ and $j$ with non-negligible coupling to the optical field are in near-resonance, $\left|\omega_{i j}\right| \approx \omega_{\mathrm{c}}$, the corresponding offdiagonal density matrix elements are transformed into a rotating reference frame,

$$
\rho_{i j}=\eta_{i j} \exp \left[-\operatorname{sgn}\left(\omega_{i j}\right) \mathrm{i} \omega_{\mathrm{c}} t\right],
$$

where sgn denotes the sign function. Inserting Eqs. 41) and (42) in Eq. (17), multiplying both sides of Eq. 17a) with $\exp \left[\operatorname{sgn}\left(\omega_{i j}\right) i \omega_{c} t\right]$ and applying the RWA, i.e., discarding all rapidly oscillating terms $\propto \exp \left( \pm \mathrm{i} \omega_{\mathrm{c}} t\right)$ and $\exp \left( \pm 2 \mathrm{i} \omega_{\mathrm{c}} t\right) \stackrel{49}{,}$ we obtain

$$
\begin{aligned}
\partial_{t} \eta_{i j}= & \mathrm{i} \Delta_{i j} \eta_{i j}+\frac{\mathrm{i}}{2 \hbar}\left(\rho_{j j}-\rho_{i i}\right) \mathbf{d}_{i j}\left\{\begin{array}{c}
\underline{\underline{\mathbf{E}}} \\
\underline{\mathbf{E}}^{*}
\end{array}\right\} \\
& -\gamma_{i j} \eta_{i j}, \omega_{i j}\left\{\begin{array}{c}
>0 \\
<0
\end{array}\right\}, \\
\partial_{t} \rho_{i i}= & \frac{1}{\hbar} \sum_{j} \Im\left\{\mathbf{d}_{j i} \eta_{i j} \underline{\mathbf{E}}^{*}\right\}+\frac{1}{\hbar} \sum_{j} \Im\left\{\mathbf{d}_{j i} \eta_{i j} \underline{\mathbf{E}}\right\} \\
& +\sum_{\omega_{i j}>0} r_{j \rightarrow i} \rho_{j j}-r_{i} \rho_{i i},
\end{aligned}
$$

with $\Delta_{i j}=\operatorname{sgn}\left(\omega_{i j}\right)\left(\omega_{\mathrm{c}}-\left|\omega_{i j}\right|\right)$. As discussed above, the RWA is only applicable if the near-resonance condition is fulfilled, i.e., all significant spectral components $E(\omega)$ of the field are close to resonance with all relevant optical transitions at frequencies $\omega_{i j},|\omega-| \omega_{i j}|| \ll\left|\omega_{i j}\right|$. As a second condition, the interaction energy must be so small that the eigenfrequencies of the quantum system are not considerably perturbed,, 49 i.e., $\left|\mathbf{d}_{i j} \underline{\mathbf{E}}\right| / \hbar \ll\left|\omega_{i j}\right|$.

\section{MAXWELL-BLOCH EQUATIONS}

The optical field propagation in the device is classically described in terms of Maxwell's equations. Assuming that the magnetization is negligible at optical frequencies, we can write Faraday's and Ampère's law for the electric field $\mathbf{E}$ and magnetic field $\mathbf{H}$ as

$$
\begin{aligned}
\nabla \times \mathbf{E} & =-\mu_{0} \partial_{t} \mathbf{H} \\
\nabla \times \mathbf{H} & =\epsilon_{0} \epsilon_{\mathrm{r}} \partial_{t} \mathbf{E}+\sigma \mathbf{E}+\mathbf{J}_{\mathrm{q}} \\
& =\epsilon_{0} \epsilon_{\mathrm{r}} \partial_{t} \mathbf{E}+\sigma \mathbf{E}+\mathbf{J}_{\mathrm{f}}+\partial_{t} \mathbf{P}_{\mathrm{q}} .
\end{aligned}
$$

$\mathbf{E}, \mathbf{H}$, and $\mathbf{J}_{\mathrm{q}}$ are functions of both $t$ and $\mathbf{x}$. $\mathbf{J}_{\mathrm{q}}=$ $\mathbf{J}_{\mathrm{f}}+\partial_{t} \mathbf{P}_{\mathrm{q}}$ denotes the total current density contribution

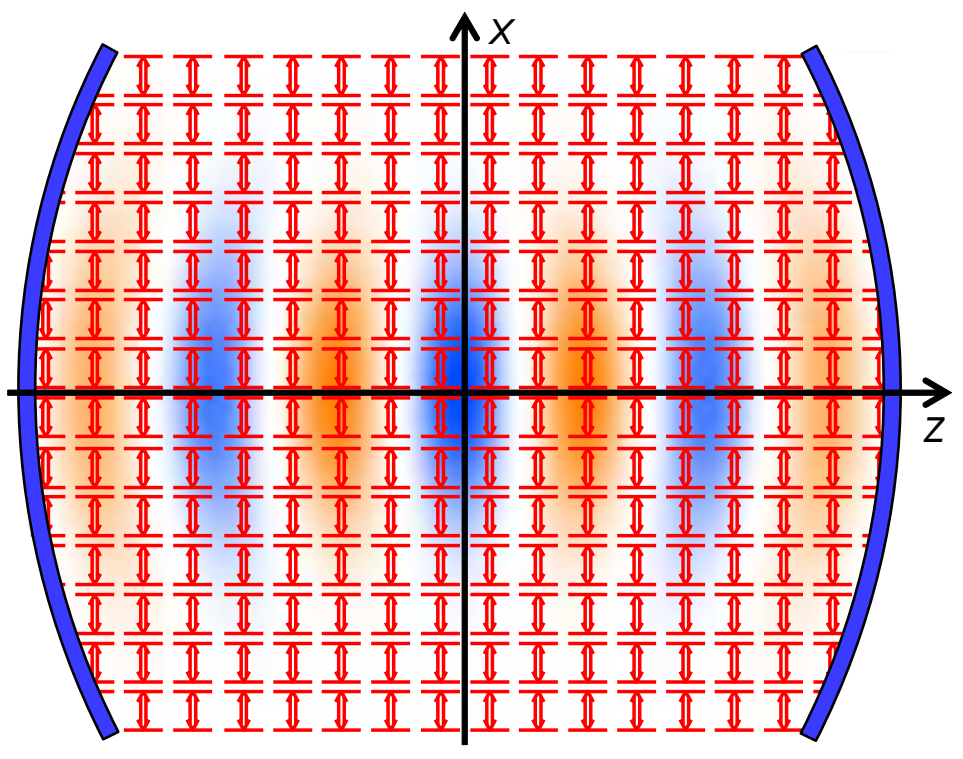

FIG. 6. Schematic illustration of modeling based on MaxwellBloch equations. For an exemplary optical resonator, the distribution of representative quantum systems is shown along with the cavity field.

of the quantum systems. Here, $\mathbf{J}_{\mathrm{f}}$ and $\partial_{t} \mathbf{P}_{\mathrm{q}}$ correspond to the current density due to free carrier motion and the polarization current density, respectively, where $\mathbf{P}_{\mathrm{q}}$ is the macroscopic polarization. In Fig. 6, the coupled modeling of the field propagation and the quantum system dynamics is schematically illustrated. For extended nanostructures such as quantum well structures or ensembles of QDs, the medium must be described by a representative quantum system at each position $\mathbf{x}$. The Bloch equations, Eq. (17), are coupled to Eq. (44) via E. On the other hand, Eq. (44) is coupled to Eq. (17) via $\mathbf{J}_{\mathrm{q}}$. For practical reasons, we consider the background polarization due to the host medium separately by the (generally $\mathbf{x}$ dependent) dielectric constant $\epsilon_{\mathrm{r}}(\mathbf{x})$, where we have for now neglected any frequency dependence, and assumed linearity and isotropy of the host. Likewise, we include the absorption of the host medium by a scalar conductivity $\sigma(\mathbf{x})$, which gives rise to an ohmic current contribution $\sigma \mathbf{E}$ in Eq. 44b. Furthermore, $\epsilon_{0}$ and $\mu_{0}$ denote the vacuum permittivity and permeability, respectively.

\section{A. Macroscopic Polarization And Current Density}

Here, as above, we use the position variable $\mathbf{r}$ to resolve microscopic behavior, while the variable $\mathbf{x}$ describes the position in the modeled device or geometry and refers to macroscopic dependencies, obtained from microscopic models by adequate ensemble averaging. In Maxwell's equations (44), the total macroscopic current density contribution of the quantum systems is given by $\mathbf{J}_{\mathrm{q}}(\mathbf{x}, t)=\mathbf{J}_{\mathrm{f}}(\mathbf{x}, t)+\partial_{t} \mathbf{P}_{\mathrm{q}}(\mathbf{x}, t)$, where the free charge current density $\mathbf{J}_{\mathrm{f}}$ and polarization current density $\partial_{t} \mathbf{P}_{\mathrm{q}}$ 
contain the contributions due to free and bound charges, respectively. In optoelectronic devices, $\mathbf{J}_{\mathrm{f}}$ is for example induced by electrical pumping or generated by the photovoltaic effect, while $\partial_{t} \mathbf{P}_{\mathrm{q}}$ is associated with the bound charge oscillations induced by the optical field. Microscopically, in nanostructured devices the carriers in bound or quasi-bound states may contribute to $\partial_{t} \mathbf{P}_{\mathrm{q}}$ by coherent or incoherent interaction with the optical field, as well as to $\mathbf{J}_{\mathrm{f}}$ via coherent transport such as tunneling and incoherent transport such as scattering-induced hopping. Thus, it makes sense to treat polarization and free current density together.

The macroscopic polarization $\mathbf{P}_{\mathrm{q}}$ can be obtained from the dipole moment of the quantum system, given by the expectation value of the dipole moment operator $\langle\hat{\mathbf{d}}\rangle(t)=\operatorname{Tr}\{\hat{\rho}(t) \hat{\mathbf{d}}\} . \mathbf{P}_{\mathrm{q}}$ at a position $\mathbf{x}$ is then obtained by summing over the quantum systems in a volume $V_{\mathrm{p}}$ around $\mathbf{x}$,

$$
\mathbf{P}_{\mathrm{q}}=V_{\mathrm{p}}^{-1} \sum_{i}\left\langle\hat{\mathbf{d}}_{i}\right\rangle,
$$

where $V_{\mathrm{p}}$ is chosen big enough to obtain a smooth dependence of $\mathbf{P}_{\mathrm{q}}$, but small enough so that spatial variations on classical length scales can still be resolved. For a large ensemble of identical systems with carrier number density $n_{3 \mathrm{D}}$, the polarization is then given by

$$
\mathbf{P}_{\mathrm{q}}=n_{3 \mathrm{D}}\langle\hat{\mathbf{d}}\rangle=n_{3 \mathrm{D}} q \operatorname{Tr}\{\hat{\mathbf{r}} \hat{\rho}\},
$$

where $\hat{\rho}$ is the density operator of a representative quantum system at position $\mathbf{x}$. On the other hand, the electric current in the quantum system can be computed from the expectation value of carrier velocity $\langle\hat{\mathbf{v}}\rangle$ in the system, where the velocity operator is defined in the Heisenberg picture by the time derivative of the position operator $\hat{\mathbf{r}}_{\mathrm{H}}, \hat{\mathbf{v}}_{\mathrm{H}}=\mathrm{d}_{t} \hat{\mathbf{r}}_{\mathrm{H}}$. For the coherent contribution corresponding to the Hamiltonian part in Eq. (3), we then obtain with the Ehrenfest equation $\langle\hat{\mathbf{v}}\rangle=\mathrm{d}_{t}\langle\hat{\mathbf{r}}\rangle, \frac{75}{, 75}$ where we have dropped the index $\mathrm{H}$ since expectation values for physical observables are independent of the chosen picture. In the Schrödinger picture, we thus obtain $\langle\hat{\mathbf{v}}\rangle=\mathrm{d}_{t} \operatorname{Tr}\{\hat{\mathbf{r}} \hat{\rho}\}=\operatorname{Tr}\left\{\hat{\mathbf{r}} \mathrm{d}_{t} \hat{\rho}\right\}$, which is also valid for the incoherent contribution induced by the Lindblad operator term in Eq. [3] ${ }^{90}$ Thus, $I=q|\langle\hat{\mathbf{v}}\rangle| / L$ corresponds to the current through an individual (single-carrier) quantum system, where $L$ here indicates the system length in the direction of current flow, and $L /|\langle\hat{\mathbf{v}}\rangle|$ is the transit time of the carrier through the system. Again averaging over a large ensemble of identical systems, we obtain the macroscopic current density $\mathbf{J}_{\mathrm{f}}=n_{3 \mathrm{D}} q \operatorname{Tr}\left\{\hat{\mathbf{r}} \mathrm{d}_{t} \hat{\rho}\right\}$. We note that this result is the same as for the polarization current density, obtained by taking the time derivative of Eq. 46), which reflects the fact that the carriers of the quantum system are responsible for both the free charge current and polarization current. Even more, from a microscopic standpoint, this distinction is inappropriate for our case. Thus we can write

$$
\mathbf{J}_{\mathrm{q}}=n_{3 \mathrm{D}} q \operatorname{Tr}\left\{\hat{\mathbf{r}} \partial_{t} \hat{\rho}\right\},
$$

where $\hat{\rho}$ again describes a representative quantum system at position $\mathbf{x}$, and $\partial_{t} \hat{\rho}$, given by Eq. (3), contains both the coherent and incoherent dynamics. Assuming an $\mathrm{N}$ level system with orthonormal basis states $|i\rangle$, Eq. (47) can with the dipole matrix element $\mathbf{d}_{i j}=q\langle i|\hat{\mathbf{r}}| j\rangle$ be written as

$$
\mathbf{J}_{\mathrm{q}}=n_{3 \mathrm{D}} \sum_{i, j} \mathbf{d}_{j i} \partial_{t} \rho_{i j}
$$

with $\partial_{t} \rho_{i j}$ given by Eq. (4) or (17).

A widely used criterion to distinguish between the macroscopic free charge and polarization current contributions in Eq. (48) is the frequency range, where commonly $\partial_{t} \mathbf{P}_{\mathrm{q}}$ is expected to contain frequencies in the range of the driving optical field spectrum, while $\mathbf{J}_{\mathrm{f}}$ covers the low-frequency and direct current contributions. In this context, we point out that due to nonlinear optical mixing, the polarization generally contains up- and down-converted components $\frac{156}{159}$ This especially applies to nanostructured optoelectronic devices where giant optical nonlinearities can be artificially engineered, and are actively exploited in both the optical and terahertz regime 160 - 163 On the other hand, the electric current can contain components up to tens of $\mathrm{GHz}$ due to external modulation or back-coupling of the optical dynamics to the electrical circuitry. Notably, in QCLs embedded into a micro-strip line, strong coupling of the copropagating microwave current modulation and optical waveform has recently been found, $164-168$ indicating that a clear differentiation between free and polarization current contributions is not always possible. However, as pointed out above, such a distinction is also not necessary since the current density and polarization appear as $\mathbf{J}_{\mathrm{q}}=\mathbf{J}_{\mathrm{f}}+\partial_{t} \mathbf{P}_{\mathrm{q}}$ in Maxwell's equations. Ultimately, the frequency range of the measured electrical current will be limited by both the measurement setup itself and the electrical properties of the device, such as its intrinsic capacitance.

\section{Coherent Contribution}

Using the Ehrenfest equation $\mathrm{d}_{t}\langle\hat{\mathbf{r}}\rangle=\mathrm{i} \hbar^{-1}\langle[\hat{H}, \hat{\mathbf{r}}]\rangle,{ }^{75}$ we can write the coherent part of the current density as

$$
\mathbf{J}_{\mathrm{coh}}=\mathrm{i} n_{3 \mathrm{D}} q \hbar^{-1}\langle[\hat{H}, \hat{\mathbf{r}}]\rangle .
$$

In the following, we assume an effective mass Hamiltonian of the form $\hat{H}=(1 / 2) \hat{\mathbf{p}}\left[m^{*}(\hat{\mathbf{r}})\right]^{-1} \hat{\mathbf{p}}+\mathbf{V}(\hat{\mathbf{r}}, t)$ as used in Eq. 201, yielding

$$
\mathbf{J}_{\mathrm{coh}}=(1 / 2) n_{3 \mathrm{D}} q\left\langle\left[m^{*}(\hat{\mathbf{r}})\right]^{-1} \hat{\mathbf{p}}+\hat{\mathbf{p}}\left[m^{*}(\hat{\mathbf{r}})\right]^{-1}\right\rangle .
$$

Using an orthonormal basis as for Eq. 48, and inserting the unit operator $\hat{I}=\int \mathrm{d} r^{3}|\mathbf{r}\rangle\langle\mathbf{r}|$ in Eq. 50 , we can ex- 
press the result in terms of wavefunctions $\varphi_{i}(\mathbf{r})=\langle\mathbf{r} \mid i\rangle$,

$\mathbf{J}_{\mathrm{coh}}=\hbar n_{3 \mathrm{D}} q \sum_{i, j} \int \Im\left\{\rho_{j i} \varphi_{i}^{*}(\mathbf{r})\left[m^{*}(\mathbf{r})\right]^{-1} \nabla \varphi_{j}(\mathbf{r})\right\} \mathrm{d}^{3} r$.

Equation (51) can also be interpreted as the current density contribution of a representative individual (singlecarrier) quantum system at the corresponding position, averaged over the associated volume $V_{\mathrm{p}}=n_{3 \mathrm{D}}^{-1}$, where the microscopically resolved current density is given by the familiar expression ${ }^{94}$

$$
\mathbf{J}_{\mathrm{coh}}=\hbar q \sum_{i, j} \Im\left\{\rho_{j i} \varphi_{i}^{*}(\mathbf{r})\left[m^{*}(\mathbf{r})\right]^{-1} \nabla \varphi_{j}(\mathbf{r})\right\} .
$$

\section{Incoherent Contribution}

The incoherent contribution to the current density is given by $\mathbf{J}_{\text {inc }}=n_{3 \mathrm{D}} q[\langle\hat{\mathbf{v}}\rangle]_{\text {inc }}=n_{3 \mathrm{D}} q \operatorname{Tr}\left\{\hat{\mathbf{r}}\left[\mathrm{d}_{t} \hat{\rho}\right]_{\text {inc }}\right\}$, which yields with Eqs. (3) and (4)

$$
\begin{aligned}
\mathbf{J}_{\text {inc }} & =n_{3 \mathrm{D}} q \operatorname{Tr}\left\{\hat{\mathbf{r}} \sum_{k}\left(\hat{L}_{k} \hat{\rho} \hat{L}_{k}^{\dagger}-\frac{1}{2} \hat{L}_{k}^{\dagger} \hat{L}_{k} \hat{\rho}-\frac{1}{2} \hat{\rho} \hat{L}_{k}^{\dagger} \hat{L}_{k}\right)\right\} \\
& =n_{3 \mathrm{D}} \sum_{i j} \mathbf{d}_{j i} \sum_{m n} \mathcal{D}_{i j m n} \rho_{m n} .
\end{aligned}
$$

For an incoherent transition from a state $\alpha$ to $\beta \neq \alpha$, we obtain with the corresponding Lindblad operator given in Eq. (7)

$\mathbf{J}_{\text {inc }}^{\alpha \rightarrow \beta}=r_{\alpha \rightarrow \beta} n_{3 \mathrm{D}}\left[\left(\mathbf{d}_{\beta \beta}-\mathbf{d}_{\alpha \alpha}\right) \rho_{\alpha \alpha}-\sum_{i \neq \alpha} \Re\left\{\mathbf{d}_{i \alpha} \rho_{\alpha i}\right\}\right]$.

Furthermore, inserting Eq. $(12)$ in Eq. $(53)$ yields the pure dephasing contribution between two levels $\alpha$ and $\beta$

$$
J_{\text {inc }}^{\alpha \beta}=-2 \gamma_{\alpha \beta}^{\prime} n_{3 \mathrm{D}} \Re\left\{\mathbf{d}_{\beta \alpha} \rho_{a \beta}\right\},
$$

with the pure dephasing rate $\gamma_{\alpha \beta}^{\prime}$. The current contributions from incoherent transitions due to Eqs. (54) and 555) can also be rearranged so that

$$
J_{\text {hop }}^{\alpha \beta}=\left(r_{\alpha \rightarrow \beta} \rho_{\alpha \alpha}-r_{\beta \rightarrow \alpha} \rho_{\beta \beta}\right) n_{3 \mathrm{D}}\left(\mathbf{d}_{\beta \beta}-\mathbf{d}_{\alpha \alpha}\right)
$$

is the net current due to the hopping transport between states $\alpha$ and $\beta$ which corresponds to the classical rate equation description, and

$$
J_{\text {dep }}^{\alpha \beta}=-2 \gamma_{\alpha \beta} n_{3 \mathrm{D}} \Re\left\{\mathbf{d}_{\beta \alpha} \rho_{a \beta}\right\}
$$

is the dephasing contribution due to the decay of the corresponding off-diagonal matrix elements $\rho_{a \beta}$ and $\rho_{\beta a}$. Here, $\gamma_{\alpha \beta}=\left(r_{\alpha}+r_{\beta}\right) / 2+\gamma_{\alpha \beta}^{\prime}$ is the total dephasing rate, including lifetime broadening and pure dephasing, and $r_{\alpha, \beta}$ is given by Eq. 10 . The total incoherent current density, resulting from incoherent transitions and pure dephasing, is then obtained by summing over all transitions. With Eqs. (56) and (57), we obtain

$$
\begin{aligned}
J_{\text {inc }}= & \sum_{\alpha=1}^{N-1} \sum_{\beta=\alpha+1}^{N}\left(J_{\text {hop }}^{\alpha \beta}+J_{\text {dep }}^{\alpha \beta}\right) \\
= & n_{3 \mathrm{D}} \sum_{\alpha} \sum_{\beta \neq \alpha}\left[r_{\alpha \rightarrow \beta} \rho_{\alpha \alpha}\left(\mathbf{d}_{\beta \beta}-\mathbf{d}_{\alpha \alpha}\right)\right. \\
& \left.-\gamma_{\alpha \beta} \Re\left\{\mathbf{d}_{\beta \alpha} \rho_{a \beta}\right\}\right] .
\end{aligned}
$$

\section{B. Slowly Varying Amplitude Approximation}

Although the Bloch equations in RWA, Eq. (43), are sometimes solved in combination with the full Maxwell's equations, Eq. (44), typically the RWA is combined with an envelope propagation equation, derived from Maxwell's equations under the assumption of a slowly varying field amplitude. In this way, above mentioned advantages of the RWA, namely a significantly reduced numerical burden and a larger number of analytical solutions, also applies to the coupled Maxwell-Bloch system. Taking the curl of Eq. (44a) and eliminating $\mathbf{H}$ using Eq. 44b) yields

$$
\begin{aligned}
\nabla \times \nabla \times \mathbf{E}= & -\frac{n_{0}^{2}\left(1-2 \Delta_{n}\right)}{c^{2}} \partial_{t}^{2} \mathbf{E}-\mu_{0} \sigma \partial_{t} \mathbf{E}-\mu_{0} \partial_{t} \mathbf{J}_{\mathrm{f}} \\
& -\mu_{0} \partial_{t}^{2} \mathbf{P}_{\mathrm{q}} .
\end{aligned}
$$

Here, $c=\left(\mu_{0} \epsilon_{0}\right)^{-1 / 2}$ is the vacuum speed of light. Furthermore, the background permittivity of the host material $\epsilon_{\mathrm{r}}(\mathbf{x})$ is here modeled as $\epsilon_{\mathrm{r}}=n_{0}^{2}\left(1-2 \Delta_{n}\right)$, where $\Delta_{n}(x, y)$ (with the minimum value 0 ) describes a transverse refractive index profile, as widely employed in waveguiding structures. $\frac{169}{}$

For no free space charges, Gauss's law dictates that $\nabla \mathbf{D}=0$ where $\mathbf{D}=\mathbf{P}_{\mathrm{q}}+\epsilon_{0} n_{0}^{2}\left(1-2 \Delta_{n}\right) \mathbf{E}$ is the displacement field in Eq. (59). Assuming an isotropic medium, we can thus set $\nabla(\nabla \mathbf{E}) \approx 0$ in the case of weak nonlinearity $\sqrt{157}$ and weak inhomogeneity, ${ }^{169 / 170}$ or generally if the field intensity transverse to the propagation direction is slowly varying over an optical wavelength. $\frac{171}{171}$ This assumption is only fulfilled for weak waveguiding, i.e., if the relative changes of the refractive index $\left|\Delta n_{\mathrm{b}}\right| / n_{\mathrm{b}}$ and its gradient $\left|\Delta\left(\nabla n_{\mathrm{b}}\right)\right| /\left|\nabla n_{\mathrm{b}}\right|$ over the distance of a wavelength in the medium is small against unity ${ }^{169}$ where $n_{\mathrm{b}}=n_{0}\left(1-2 \Delta_{n}\right)^{1 / 2}$ in Eq. 599. Furthermore, also the polarization contribution $\overline{\mathbf{P}}_{\mathrm{q}}$ of the quantum structure must be compatible with the assumption of weak inhomogeneity. As discussed in Section IIIC, quantum structures, as modeled by the Bloch equations, can be highly anisotropic; e.g., the dipole moment element vector $\mathbf{d}_{i j}$ of inter-conduction band transitions in quantum wells only has a nonzero component in growth direction. If the optical field is however also polarized in 
this direction, which is for example often the case in lasers since only the corresponding field component gets amplified, then $\nabla \mathbf{E} \approx 0$ can still hold for weak nonlinearity and inhomogeneity. Using $\nabla \times(\nabla \times \mathbf{E})=\nabla(\nabla \mathbf{E})-\nabla^{2} \mathbf{E}$ and subsequently neglecting the term $\nabla(\nabla \mathbf{E})$, we obtain the generalized inhomogeneous wave equation

$$
\nabla^{2} \mathbf{E}=\frac{n_{0}^{2}\left(1-2 \Delta_{n}\right)}{c^{2}} \partial_{t}^{2} \mathbf{E}+\mu_{0} \sigma \partial_{t} \mathbf{E}+\mu_{0} \partial_{t} \mathbf{J}_{\mathrm{f}}+\mu_{0} \partial_{t}^{2} \mathbf{P}_{\mathrm{q}} .
$$

For deriving the slowly varying amplitude approximation (SVAA), $\mathbf{E}$ and $\mathbf{P}_{\mathrm{q}}$ are written as a product of its envelope and carrier, as done above for the derivation of the RWA. However, in contrast to Eq. (41), we also take into account the spatial dependence of the carrier, where we assume that the direction of the optical energy flow at every position is close to a reference direction defined by the carrier wavevector $\mathbf{k}_{\mathrm{c}}$, which corresponds to the paraxial approximation. This assumption is for example typically fulfilled in laser resonators or optical fibers. Introducing the complex-valued field and polarization amplitudes, $\underline{\mathbf{E}}(\mathbf{x}, t)$ and $\underline{\mathbf{P}}(\mathbf{x}, t)$, and assuming propagation along the $z$ direction, we have

$$
\begin{aligned}
\mathbf{E}(\mathbf{x}, t) & =\frac{1}{2} \underline{\mathbf{E}}(\mathbf{x}, t) \exp \left(\mathrm{i} k_{\mathrm{c}} z-\mathrm{i} \omega_{\mathrm{c}} t\right)+c . c . \\
\mathbf{P}_{\mathrm{q}}(\mathbf{x}, t) & =\frac{1}{2} \underline{\mathbf{P}}(\mathbf{x}, t) \exp \left(\mathrm{i} k_{\mathrm{c}} z-\mathrm{i} \omega_{\mathrm{c}} t\right)+c . c .
\end{aligned}
$$

with $\left|k_{\mathrm{c}}\right|=n_{0} \omega_{\mathrm{c}} / c$. We note that although Eq. 61a contains the term $\exp \left(\mathrm{i} k_{\mathrm{c}} z\right)$ not included in Eq. (41), the Bloch equations in RWA, Eq. 43, remain unchanged since $\exp \left(\mathrm{i} k_{\mathrm{c}} z\right)$ cancels out. To apply the SVAA, we insert Eq. 61) in Eq. (60). Just as for the RWA, we assume that all significant spectral components of the field are close to $\omega_{\text {c }}$, i.e., at frequencies $\omega_{\mathrm{c}}+\Delta_{\omega}$ with $\left|\Delta_{\omega}\right| \ll$ $\omega_{\mathrm{c}}$. This implies that $\partial_{t}^{2} \underline{\mathbf{E}}$ can be neglected against $-2 \mathrm{i} \omega_{\mathrm{c}} \partial_{t} \underline{\mathbf{E}}$, as can be seen in Fourier domain where the two terms become $-\Delta_{\omega}^{2} \underline{\mathbf{E}}\left(\Delta_{\omega}\right)$ and $-2 \omega_{\mathrm{c}} \Delta_{\omega} \underline{\mathbf{E}}\left(\Delta_{\omega}\right)$. Similarly, also $\sigma \partial_{t} \mathbf{E}$ and $\Delta_{n} \partial_{t} \mathbf{E}$ can be dropped against $-\mathrm{i} \omega_{\mathrm{c}} \sigma \underline{\mathbf{E}}$ and $-\mathrm{i} \omega_{\mathrm{c}} \Delta_{n} \mathbf{E}$. The polarization amplitude $\underline{\mathbf{P}}$, introduced in Eq. (61b), couples the optical propagation equation to the Bloch equations, Eq. 43, as further discussed in Section IVB 1. The RWA implies that also $\underline{\mathbf{P}}$ is narrowband, which means that for example harmonic or difference frequency generation cannot be included. Thus, similarly as for the field, $\partial_{t}^{2} \underline{\mathbf{P}}$ and $-2 \mathrm{i} \omega_{\mathrm{c}} \partial_{t} \underline{\mathbf{P}}$ can be neglected against $-\omega_{\mathrm{c}}^{2} \underline{\mathbf{P}}$. In addition, the paraxial approximation implies that $\partial_{z}^{2} \underline{\mathbf{E}}$ can be neglected against $\mathrm{i} k_{\mathrm{c}} \partial_{z} \mathbf{E}$. Finally multiplying all terms with $\exp \left(\mathrm{i} \omega_{\mathrm{c}} t-\mathrm{i} k_{\mathrm{c}} z\right)$ and discarding all rapidly oscillating terms, which also eliminates $\mathbf{J}_{\mathrm{f}}$ since it is assumed to contain only low frequency components (see Section IV A, , we arrive at

$$
\begin{aligned}
\partial_{t} \underline{\mathbf{E}} \pm \frac{c}{n_{0}} \partial_{z} \underline{\mathbf{E}}= & -\mathrm{i} \omega_{\mathrm{c}} \Delta_{n} \underline{\mathbf{E}} \\
& +\frac{1}{2 n_{0}^{2}}\left(\mathrm{i} \frac{c^{2}}{\omega_{\mathrm{c}}} \nabla_{\mathrm{T}}^{2} \underline{\mathbf{E}}+\mathrm{i} \frac{\omega_{\mathrm{c}}}{\epsilon_{0}} \underline{\mathbf{P}}-\frac{\sigma}{\epsilon_{0}} \underline{\mathbf{E}}\right) .
\end{aligned}
$$

Here, $\nabla_{\mathrm{T}}^{2}=\partial_{x}^{2}+\partial_{y}^{2}$ denotes the transverse Laplace operator. The "+" and "-" signs in Eq. [62 are for forward and backward propagation corresponding to $k_{\mathrm{c}}>0$ and $k_{\mathrm{c}}<0$, respectively. For counterpropagating fields which for example arise in Fabry-Pérot resonators, the standing wave pattern causes a position dependent inversion grating, also referred to as spatial hole burning. This effect is not yet included in Eq. (62), and its implementation is discussed in Section $\mathrm{VC} 2$

\section{Polarization in Rotating Wave Approximation}

In the RWA, the off-diagonal density matrix elements $\rho_{i j}$ that are associated with near-resonant optical transitions are represented in terms of transformed elements $\eta_{i j}$ in a rotating reference frame, as obtained with Eq. 42 . Writing the total current as $\mathbf{J}_{\mathrm{q}}=\mathbf{J}_{\mathrm{f}}+\partial_{t} \mathbf{P}_{\mathrm{q}}$ as in Eq. (47), and assigning the low-frequency contributions to $\mathbf{J}_{\mathrm{f}}$ and the optical contributions to $\mathbf{P}_{\mathrm{q}}$, we see from Eq. 48 that the transformation into the rotating frame only affects the evaluation of the polarization $\mathbf{P}_{\mathrm{q}}$. With Eq. 46 and Eq. 42, we obtain

$\mathbf{P}_{\mathrm{q}}=n_{3 \mathrm{D}} \sum_{\omega_{i j}>0} \mathbf{d}_{j i} \eta_{i j} \exp \left(-\mathrm{i} \omega_{\mathrm{c}} t\right)+$ c.c. $+n_{3 \mathrm{D}} \sum_{i} \mathbf{d}_{i i} \rho_{i i}$.

For inclusion of optical propagation, the RWA is often not coupled to the full Maxwell equations, but rather solved together with Eq. 62 in SVAA which contains the polarization in terms of the amplitude $\underline{\mathbf{P}}$. As discussed above, we have to replace $\exp \left(-\mathrm{i} \omega_{\mathrm{c}} t\right)$ by $\exp \left(\mathrm{i} k_{\mathrm{c}} z-\mathrm{i} \omega_{\mathrm{c}} t\right)$ in Eq. (63) since the SVAA also takes into account the spatial dependence of the carrier. Comparing the resulting equation with Eq. (61b), and neglecting the quasi-static dipole moment contribution $\sum_{i} \mathbf{d}_{i i} \rho_{i i}$ which does not oscillate at the optical excitation frequency and thus drops out in the SVAA, we obtain

$$
\underline{\mathbf{P}}=2 n_{3 \mathrm{D}} \sum_{\omega_{i j}>0} \mathbf{d}_{j i} \eta_{i j} .
$$

\section{Initial Conditions}

The Bloch equations without or with RWA, Eq. 17) or Eq. (43), have to be supplemented by corresponding initial conditions at time $t=t_{0}$. Apart from special cases where the quantum system may be coherently prepared in a certain initial state such as a coherent superposition,, 172 the system will be initially in equilibrium. The corresponding density matrix elements are then obtained by setting $\partial_{t}=0$ in Eq. 177 or (43) and assuming a vanishing optical field for $t \leq t_{0}$, which gives rise to a mixed state with off-diagonal elements $\rho_{i j}\left(t=t_{0}\right)=0$ and $\eta_{i j}\left(t=t_{0}\right)=0$, respectively. The diagonal elements $\rho_{i i}\left(t=t_{0}\right)=\rho_{i i}^{\text {eq }}$ are given by the 
equilibrium occupation probabilities $\rho_{i i}^{\mathrm{eq}}$, which can be obtained by setting $\mathrm{d}_{t}=0$ in Eq. (9). This yields for a system with $N$ levels the linear equation system

$$
\begin{aligned}
0 & =\sum_{j \neq i} r_{j \rightarrow i} \rho_{j j}^{\mathrm{eq}}-r_{i} \rho_{i i}^{\mathrm{eq}}, i=1 . .(N-1), \\
1 & =\sum_{i=1}^{N} \rho_{i i}^{\mathrm{eq}},
\end{aligned}
$$

where $r_{i}$ is given by Eq. 10]. The $\rho_{i i}^{\mathrm{eq}}$ do not necessarily correspond to a thermal distribution, but are rather determined by the transition rates which may for example include the pumping process in lasers. For inhomogeneous device structures, the rates $r_{i}$ and $r_{j \rightarrow i}$ generally depend on position $\mathbf{x}$, giving rise to $\mathbf{x}$ dependent $\rho_{i i}^{\mathrm{eq}}$.

Suitable initial conditions have also to be defined for the Maxwell equations, Eq. (44), or the propagation equations in SVAA derived thereof, Eq. (62). Here we cannot choose identically vanishing fields, since the optical field would then remain zero throughout the $\mathrm{MB}$ simulation. Laser seeding by spontaneous emission is often mimicked by initializing the electric field with white Gaussian amplitude noise, which can also be added at every time step of the simulation to model spontaneous emission noise ${ }^{173}$ In the SVAA, Eq. 62), the electric field is represented by its complex envelope function, and thus complex white Gaussian noise is used in this case.

\section{Two-Level Approximation}

In most cases, the simplest model with only two relevant states (e.g., an upper laser level 2 and lower laser level 1) is considered. The corresponding density matrix contains the elements $\rho_{11}, \rho_{22}$ and $\rho_{21}=\rho_{12}^{*}$. Assuming a closed system, we obtain $\rho_{11}+\rho_{22}=1$. The dissipation in the Bloch equations, Eq. (17), is then parametrized by the three rates $\gamma_{21}, r_{1 \rightarrow 2}$ and $r_{2 \rightarrow 1}$, where $\gamma_{12}=\gamma_{21}$ as described in Section IIB 2 and Eq. (10) gives $r_{1}=$ $r_{1 \rightarrow 2}, r_{2}=r_{2 \rightarrow 1}$. Introducing the population inversion $w=\rho_{22}-\rho_{11}$, we can substitute $\rho_{11}=(1-w) / 2$ and $\rho_{22}=(1+w) / 2$ in Eq. 17). Furthermore neglecting static dipole moments, $\mathbf{d}_{22}-\mathbf{d}_{11} \approx \mathbf{0}$, we obtain

$$
\begin{aligned}
\partial_{t} \rho_{21} & =-\mathrm{i} \omega_{21} \rho_{21}-\mathrm{i} w \Omega-\gamma_{2} \rho_{21}, \\
\partial_{t} w & =2 \mathrm{i}\left(\rho_{21}^{*} \Omega-\rho_{21} \Omega^{*}\right)-\gamma_{1} w+\Gamma_{12} .
\end{aligned}
$$

Here, $\Omega=\hbar^{-1} \mathbf{d}_{21} \mathbf{E}$ denotes the instantaneous Rabi frequency, $\gamma_{1}=r_{1 \rightarrow 2}+r_{2 \rightarrow 1}$ and $\gamma_{2}=\gamma_{21}$ are the population inversion relaxation and dephasing rates, and $\Gamma_{12}=r_{1 \rightarrow 2}-r_{2 \rightarrow 1}$ represents the (net) pumping rate from the lower to the upper level. Equation $67 \mathrm{~b}$ ) is often also written as

$$
\partial_{t} w=2 \mathrm{i}\left(\rho_{21}^{*} \Omega-\rho_{21} \Omega^{*}\right)-\gamma_{1}\left(w-w_{\text {eq }}\right),
$$

where $w_{\text {eq }}=\Gamma_{12} / \gamma_{1}$ denotes the equilibrium population inversion for $\Omega=0$.

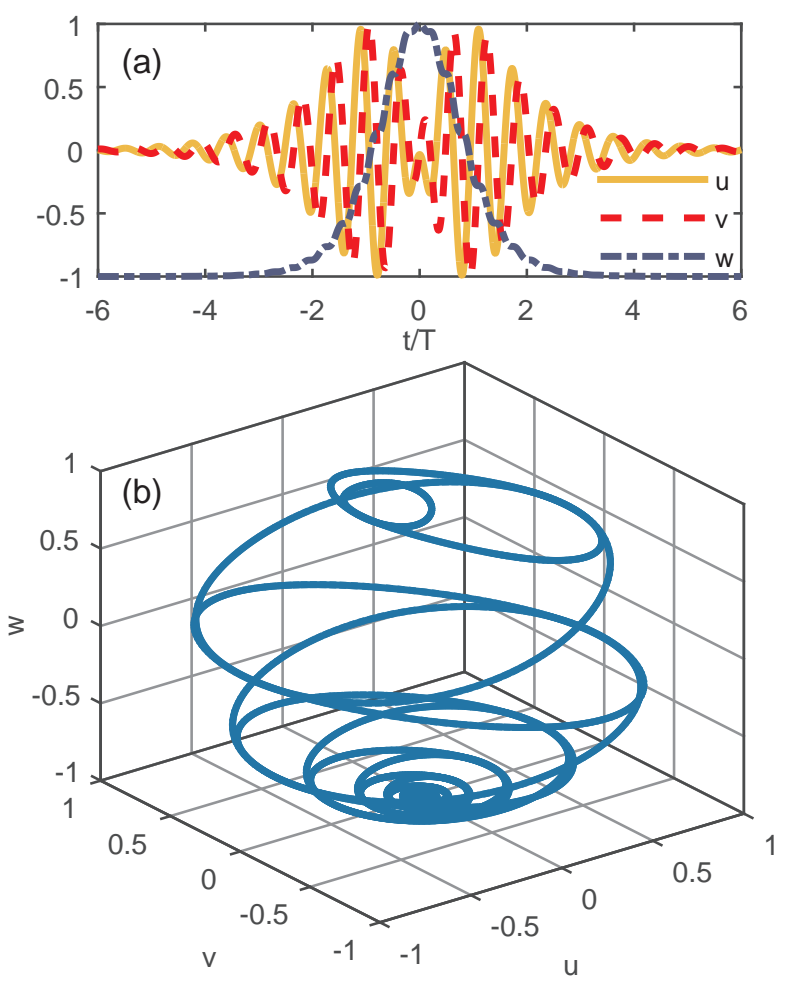

FIG. 7. (a) Bloch vector components and (b) Bloch vector trajectory for a dissipationless two-level system excited with a sech pulse.

A real-valued, redundance-free representation can be obtained by applying Eqs. (27) and (28), yielding the three real-valued quantities $u:=\left\langle\hat{u}_{12}\right\rangle=\rho_{12}+\rho_{21}=$ $2 \Re\left\{\rho_{21}\right\}, v:=-\left\langle\hat{v}_{12}\right\rangle=-\mathrm{i}\left(\rho_{12}-\rho_{21}\right)=-2 \Im\left\{\rho_{21}\right\}$, and $w:=\left\langle\hat{w}_{1}\right\rangle=\rho_{22}-\rho_{11}$. These are usually represented in terms of the so-called Bloch vector $\mathbf{S}=[u, v, w]^{\mathrm{T}}$, where a minus sign has been added to the definition of $v$ in order to obtain the usual convention for the Bloch vector $\stackrel{49}{4}$ Separating $\Omega$ in its real and imaginary part $\Omega_{\mathrm{r}}+\mathrm{i} \Omega_{\mathrm{i}}$, Eq. 67) then becomes

$$
\begin{aligned}
\partial_{t} u & =-\omega_{21} v+2 \Omega_{\mathrm{i}} w-\gamma_{2} u, \\
\partial_{t} v & =\omega_{21} u+2 \Omega_{\mathrm{r}} w-\gamma_{2} v, \\
\partial_{t} w & =-2 \Omega_{\mathrm{i}} u-2 \Omega_{\mathrm{r}} v-\gamma_{1} w+\Gamma_{12},
\end{aligned}
$$

which can also be written as 49

$$
\partial_{t} \mathbf{S}=\left(\begin{array}{c}
-2 \Omega_{\mathrm{r}} \\
2 \Omega_{\mathrm{i}} \\
\omega_{21}
\end{array}\right) \times \mathbf{S}-\left(\begin{array}{c}
\gamma_{2} u \\
\gamma_{2} v \\
\gamma_{1}\left(w-w_{\mathrm{eq}}\right)
\end{array}\right) .
$$

The polarization term in Eq. 444 is then with Eq. 48 obtained as

$$
\begin{aligned}
\partial_{t} \mathbf{P}_{\mathrm{q}} & =2 n_{3 \mathrm{D}} \Re\left\{\mathbf{d}_{12} \partial_{t} \rho_{21}\right\} \\
& =n_{3 \mathrm{D}}\left(\Re\left\{\mathbf{d}_{12}\right\} \partial_{t} u+\Im\left\{\mathbf{d}_{12}\right\} \partial_{t} v\right) .
\end{aligned}
$$

The time evolution of the Bloch vector $\mathbf{S}(t)$ can be visualized in the Bloch sphere representation, where the 
Bloch vector trajectory is displayed in a Cartesian coordinate system with axes $u, v$ and $w ! \frac{47 / 49}{}$ For $\gamma_{1}=\gamma_{2}=0$, $|\mathbf{S}(t)|$ is conserved over time, as can be seen by multiplying Eqs. 69a), 69b and 69c with $u, v$ and $w$, respectively, and adding the resulting equations, which yields $\partial_{t}\left(u^{2}+v^{2}+w^{2}\right)=0$. For pure states, $|\mathbf{S}(t)|=1$, i.e., the tip of the Bloch vector moves along the surface of a unit sphere, the so-called Bloch sphere. For mixed states, the tip is located within the Bloch sphere, corresponding to $|\mathbf{S}(t)|<1$. In Fig.7 7, the time evolution of the Bloch vector components and the corresponding Bloch vector trajectory are shown for a two-level system with $\gamma_{1}=\gamma_{2}=0$ and initial conditions $u=v=0$, $w=-1$. The optical field is assumed to be a sech pulse $\Omega=2 T^{-1} \operatorname{sech}(t / T)$, which corresponds to a selfinduced transparency soliton as further discussed in Section VI A 2, with $T=10 / \omega_{21}$ chosen for this example.

A further representation of the Bloch equations is obtained by assuming a real $\mathbf{d}_{21}$ and thus $\Omega=\Omega_{\mathrm{r}}$. Solving Eq. 69a) for $v$ and using the result to eliminate $v$ in Eqs. $69 \mathrm{~b}$ and $69 \mathrm{c}$ yields with Eq. $71{ }^{157}$

$$
\left[\partial_{t}^{2}+2 \gamma_{2} \partial_{t}+\left(\omega_{21}^{2}+\gamma_{2}^{2}\right)\right] \mathbf{P}_{\mathrm{q}}=-\frac{2 \omega_{21} \mathbf{d}_{12}^{2}}{\hbar} n_{3 \mathrm{D}} w \frac{\mathbf{d}_{12}}{\left|\mathbf{d}_{12}\right|} \mathbf{E},
$$

$$
\partial_{t} w=2 \frac{\partial_{t} \mathbf{P}_{\mathrm{q}}+\gamma_{2} \mathbf{P}_{\mathrm{q}}}{\hbar n_{3 \mathrm{D}} \omega_{21}} \mathbf{E}-\gamma_{1} w+\Gamma_{12} .
$$

This representation can be seen as an extension of the classical Lorentz model for resonant polarization in dielectrics, assuming the same mathematical form as Eq. (72a) if we set $w$ constant. Accordingly, Eq. 72 . is mainly used in computational electrodynamics, especially in combination with the finite-difference timedomain method, as a substitute for more basic classical polarization models $[52$

\section{Rotating Wave/Slowly Varying Amplitude Approximation}

In the RWA, we obtain from Eq. (43) with $\Omega=$ $\hbar^{-1} \mathbf{d}_{21} \underline{\mathbf{E}}$

$$
\begin{aligned}
\partial_{t} \eta_{21} & =\mathrm{i} \Delta \eta_{21}-\frac{1}{2} \mathrm{i} w \underline{\Omega}-\gamma_{2} \eta_{21}, \\
\partial_{t} w & =\mathrm{i}\left(\eta_{21}^{*} \underline{\Omega}-\eta_{21} \underline{\Omega}^{*}\right)-\gamma_{1}\left(w-w_{\mathrm{eq}}\right),
\end{aligned}
$$

where $\Delta=\omega_{\mathrm{c}}-\omega_{21}$ denotes the detuning of the optical field from the resonance frequency $\omega_{21}$. In analogy to above, we can introduce the Bloch vector $\mathbf{s}$ for the off-diagonal density matrix elements in RWA, with components $s_{1}=\eta_{12}+\eta_{21}=2 \Re\left\{\eta_{21}\right\}, s_{2}=-\mathrm{i}\left(\eta_{12}-\eta_{21}\right)=$ $-2 \Im\left\{\eta_{21}\right\}, s_{3}=w$, and obtain in analogy to Eq. (69) with $\underline{\Omega}=\underline{\Omega}_{\mathrm{r}}+\mathrm{i} \underline{\Omega}_{\mathrm{i}}$

$$
\begin{aligned}
\partial_{t} s_{1} & =\Delta s_{2}+\underline{\Omega}_{\mathrm{i}} w-\gamma_{2} s_{1} \\
\partial_{t} s_{2} & =-\Delta s_{1}+\underline{\Omega}_{\mathrm{r}} w-\gamma_{2} s_{2} \\
\partial_{t} w & =-\underline{\Omega}_{\mathrm{i}} s_{1}-\underline{\Omega}_{\mathrm{r}} s_{2}-\gamma_{1}\left(w-w_{\mathrm{eq}}\right) .
\end{aligned}
$$

The polarization term in the SVAA propagation equation, Eq. (62), is then with Eq. (64) obtained as

$$
\underline{\mathbf{P}}=2 n_{3 \mathrm{D}} \mathbf{d}_{12} \eta_{21}=n_{3 \mathrm{D}} \mathbf{d}_{12}\left(s_{1}-\mathrm{i} s_{2}\right) .
$$

\section{REDUCTION TO ONE-DIMENSIONAL MODEL}

Although the MB equations are sometimes solved in two or even three spatial dimensions, 53,60 the model is frequently reduced to a single spatial coordinate in order to minimize the numerical load $\frac{49}{\text { This is usually }}$ achieved by assuming plane wave propagation in the Maxwell equations, Eq. 44), or the corresponding propagation equations in SVAA, Eq. (62) [174 For extended beams propagating in a homogeneous medium such as a gas or bulk solid-state medium, the plane wave approximation may be a reasonable assumption. For optoelectronic devices which are the focus of this paper, the light is usually strongly guided, often with subwavelength confinement in at least one dimension. Here, the plane wave approximation is clearly too simplistic. However, optoelectronic devices such as semiconductorbased lasers often employ waveguiding structures which are invariant in propagation direction $z$, in particular schemes with a suitable transverse refractive index profile or metal cladding. Such geometries provide lateral field confinement and give rise to guided mode solutions, i.e., field solutions which are at a given frequency $\omega$ characterized by a propagation constant and a $z$ independent transverse field distribution. While some one-dimensional plane wave treatments have included all transverse field components to describe elliptically or circularly polarized light, $, 152|153| 175,177]$ we assume linearly polarized waveguide modes in the following, and thus consider a single transverse component of the electric and magnetic fields. In Fig. 8, an exemplary waveguide structure is schematically illustrated.

\section{A. Full Maxwell Equations}

We employ the full Maxwell equations, Eq. 44, coupled to the Bloch equations, Eq. (16) or (17), to describe the carrier-light interaction and optical propagation in a waveguide geometry which is invariant with respect to the propagation direction $z$. Our goal is to extract a one-dimensional MB model with a single electric and magnetic field component, as typically used in simulations due to the associated computational burden. We focus on guided mode solutions, which are at a given frequency $\omega$ characterized by a (generally complex) propagation constant $\beta$, and $z$ invariant transverse field dependencies $\underline{E}_{x, y, z}^{\mathrm{t}}(x, y)$ and $\underline{H}_{x, y, z}^{\mathrm{t}}(x, y)$ for the electric and magnetic field components. Thus, we can make the 


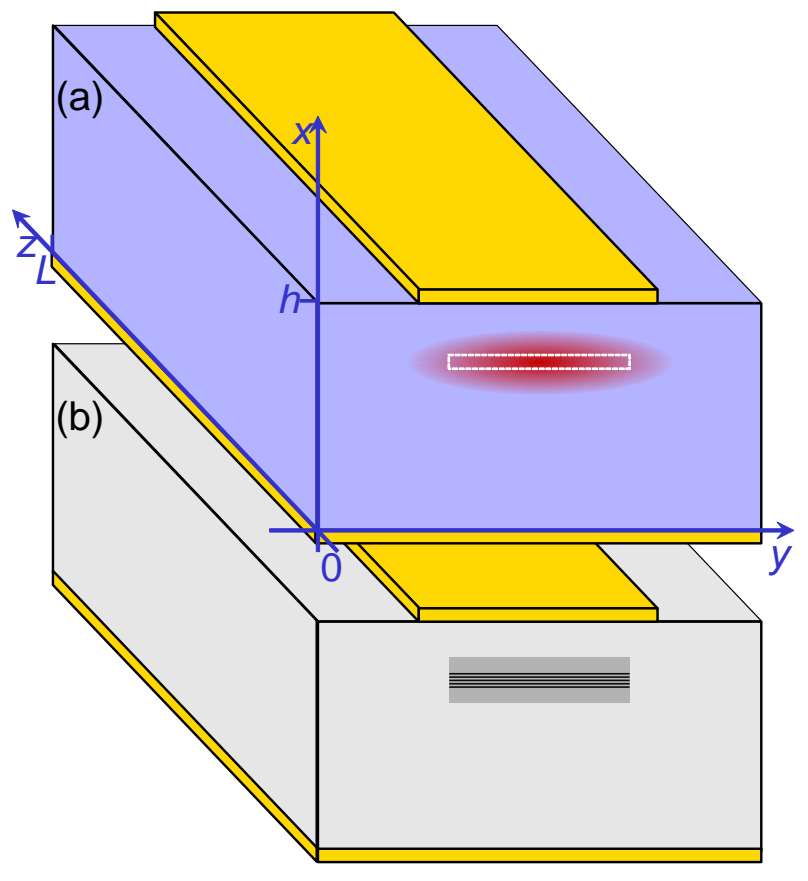

FIG. 8. Schematic illustration of one-dimensional propagation model for a waveguide structure. (a) The white rectangle on the front facet denotes the cross section of the quantum structure, and the optical intensity distribution is indicated. (b) The refractive index profile is indicated, with darker colors corresponding to higher refractive indices.

ansatz

$$
\begin{aligned}
E_{p}(\mathbf{x}, t) & =\Re\left\{\underline{E}_{p}^{\mathrm{t}}(x, y) \exp (\mathrm{i} \underline{\beta} z-\mathrm{i} \omega t)\right\} \\
& =\Re\left\{\underline{E}_{p}^{\omega}(\mathbf{x}) \exp (-\mathrm{i} \omega t)\right\}, \\
H_{p}(\mathbf{x}, t) & =\Re\left\{\underline{H}_{p}^{\mathrm{t}}(x, y) \exp (\mathrm{i} \underline{\beta} z-\mathrm{i} \omega t)\right\} \\
& =\Re\left\{\underline{H}_{p}^{\omega}(\mathbf{x}) \exp (-\mathrm{i} \omega t)\right\},
\end{aligned}
$$

with $p=x, y, z$. By inserting Eq. (76) into Eq. (44), the computation of the transverse mode profile in the $x y$ plane can be decoupled from the $z$ coordinate and reduces to a two-dimensional problem. For example, by eliminating the electric field, we obtain

$$
\left(\partial_{p}^{2}+\partial_{q}^{2}\right) \underline{H}_{p}^{\mathrm{t}}+\frac{\partial_{q} \underline{\epsilon}_{\mathrm{r}}}{\underline{\epsilon}_{\mathrm{r}}}\left(\partial_{p} \underline{H}_{q}^{\mathrm{t}}-\partial_{q} \underline{H}_{p}^{\mathrm{t}}\right)=\left(\underline{\beta}^{2}-\frac{\omega^{2}}{c^{2}} \underline{\epsilon}_{\mathrm{r}}\right) \underline{H}_{p}^{\mathrm{t}}
$$

with $p=x, y$ and $q=y, x !^{178}$ Waveguiding is for instance obtained by surrounding the optically active region with another dielectric material featuring a lower refractive index as illustrated in Fig.8(b), or with a metal cladding. Both cases can be described by a transversely dependent complex background permittivity

$$
\underline{\epsilon}_{\mathrm{r}}=\epsilon_{\mathrm{r}}+\mathrm{i} \sigma /\left(\omega \epsilon_{0}\right),
$$

where $\epsilon_{\mathrm{r}}$ and $\sigma$ generally depend on $x, y$ and $\omega$, and $\sigma$ accounts for the conductivity or dielectric losses. Together with the boundary condition $\underline{H}_{p, q}^{\mathrm{t}} \rightarrow 0$ for $x^{2}+y^{2} \rightarrow$ $\infty$, Eq. (77) constitutes a complex eigenvalue problem. Equation (77) can for example be solved with the film mode matching method, which is especially suitable for waveguides with a rectangular cross section. ${ }^{[79}$ The polarization contribution $\mathbf{P}_{\mathrm{q}}$ in Eq. 44 due to the quantum systems is not yet considered in Eq. (77) since it is assumed to be small enough to be included in firstorder perturbation theory, with negligible influence on the transverse field distribution. Using $\nabla \mathbf{H}=0$, we can calculate the longitudinal component $\underline{H}_{z}^{\mathrm{t}}(x, y)$ from $\underline{H}_{x}^{\mathrm{t}}$ and $\underline{H}_{y}^{\mathrm{t}}$ as

$$
\underline{H}_{z}^{\mathrm{t}}=\mathrm{i} \underline{\beta}^{-1}\left(\partial_{x} \underline{H}_{x}^{\mathrm{t}}+\partial_{y} \underline{H}_{y}^{\mathrm{t}}\right) .
$$

Furthermore, the electric field components are with Eq. 44b obtained as

$$
\begin{aligned}
& \omega \epsilon_{0} \underline{\epsilon}_{\mathrm{r}} \underline{E}_{x}^{\mathrm{t}}=\mathrm{i} \partial_{y} \underline{H}_{z}^{\mathrm{t}}+\beta \underline{H}_{y}^{\mathrm{t}}, \\
& \omega \epsilon_{0} \underline{\epsilon}_{\mathrm{r}} \underline{E}_{y}^{\mathrm{t}}=-\underline{\beta}_{x}^{\mathrm{t}}-\mathrm{i} \partial_{x} \underline{H}_{z}^{\mathrm{t}}, \\
& \omega \epsilon_{0} \underline{\epsilon}_{\mathrm{r}} \underline{E}_{z}^{\mathrm{t}}=\mathrm{i} \partial_{x} \underline{H}_{y}^{\mathrm{t}}-\mathrm{i} \partial_{y} \underline{H}_{x}^{\mathrm{t}} .
\end{aligned}
$$

For general solutions of Eq. (77), the polarization varies over the waveguide cross section. As indicated in Fig. 8(b), in many optoelectronic devices, such as typical standard edge-emitting and quantum cascade lasers, rectangular waveguides are used where the width in lateral $y$ direction significantly exceeds its thickness in $x$ direction. This allows an approximate treatment as a slab waveguide structure, which is assumed to be infinitely extended in $y$ direction and thus can, to first order, be described by $\underline{\epsilon}_{\mathrm{r}}(x)$. The field components are then assumed to be constant in $y$ direction, which corresponds to setting $\partial_{y}=0.170$ The guided field solutions can be divided into two classes: Transverse electric (TE) modes are characterized by $\underline{E}_{z}^{\omega}=0$, where for $\partial_{y}=0$ all components except $\underline{E}_{y}^{\omega}, \underline{H}_{x}^{\omega}$ and $\underline{H}_{z}^{\omega}$ vanish as can be seen from Eqs. (79) and (80); similarly, transverse magnetic (TM) modes, characterized by $\underline{H}_{z}^{\omega}=0$, have for $\partial_{y}=0$ only non-vanishing $\underline{H}_{y}^{\omega}, \underline{E}_{x}^{\omega}$ and $\underline{E}_{z}^{\omega}$ components. 170 The $y$ dependence of the field distribution may then be reintroduced using the effective refractive index approximation method, 180 which preserves the TE or TM character of the solution. From the discussion of dipole matrix elements for quantum well structures in Section IIIC, it follows that standard edge-emitting lasers, which utilize interband transitions, preferably operate in TE mode [see also Fig.5(a)]. On the other hand, QCLs, which rely on intraband transitions, only operate in TM mode [see also Fig. $5(\mathrm{c})]$.

As pointed out above, simulations typically employ a plane-wave-type propagation model which only depends on the propagation coordinate $z$ and time $t$, and considers a single transverse electric and transverse magnetic field component. Our goal is to derive such equations, with a form equivalent to the Maxwell equations, for guided rather than plane-wave propagation, as applies to many photonic devices and systems. 


\section{Transverse Electric Mode}

For TE modes in slab waveguides, Eq. (77) yields with $\underline{H}_{y}^{\mathrm{t}}=0$ and $\partial_{y}=0$

$$
\partial_{x}^{2} \underline{H}_{x}^{\mathrm{t}}=\left(\underline{\beta}^{2}-\frac{\omega^{2}}{c^{2}} \underline{\epsilon}_{\mathrm{r}}\right) \underline{H}_{x}^{\mathrm{t}}
$$

and the boundary conditions are given by $\underline{H}_{x}^{\mathrm{t}}(x \rightarrow \pm \infty) \rightarrow 0$. From Eq. 44 , we furthermore obtain

$$
\begin{aligned}
-\partial_{z} E_{y} & =-\mu_{0} \partial_{t} H_{x} \\
\partial_{z} H_{x}-\partial_{x} H_{z} & =\epsilon_{0} \epsilon_{\mathrm{r}} \partial_{t} E_{y}+\sigma E_{y}
\end{aligned}
$$

The polarization contribution of the quantum systems is not contained in Eq. $82 \mathrm{~b}$ since it will subsequently be included in a perturbative manner. Equation (82b) does not yet have the desired form since it contains an $x$ derivative and the longitudinal field component in the term $\partial_{x} H_{z}$. With Eqs. (76b), 79) and (81), we obtain

$$
\partial_{x} \underline{H}_{z}^{\omega}=\mathrm{i} \underline{\beta}^{-1}\left(\underline{\beta}^{2}-\frac{\omega^{2}}{c^{2}} \underline{\epsilon}_{\mathrm{r}}\right) \underline{H}_{x}^{\omega},
$$

where $\underline{\epsilon}_{\mathrm{r}}=\epsilon_{\mathrm{r}}+\mathrm{i} \sigma /\left(\omega \epsilon_{0}\right)$. In the following, it is practical to switch to the frequency domain, where Eq. (82a) is with Eq. 76 given by

$$
\partial_{z} \underline{E}_{y}^{\omega}=\mathrm{i} \underline{\beta} \underline{E}_{y}^{\omega}=-\mathrm{i} \omega \mu_{0} \underline{H}_{x}^{\omega} .
$$

From Eq. 84, we see that the electric and magnetic fields have the same transverse distribution. Inserting Eq. 83) into Eq. (82b) in frequency domain, and employing Eq. 84, we arrive at

$$
\partial_{z} \underline{H}_{x}^{\omega}=-\mathrm{i} \omega^{-1} \mu_{0}^{-1} \underline{\beta}^{2} \underline{E}_{y}^{\omega}
$$

In the following, the polarization contribution of the quantum system will be included as a perturbation 181 Re-deriving Eq. (81) from Maxwell's equations Eq. 44, but now with the polarization contribution due to the quantum systems included, we see that the perturbation generated by the polarization on the $\underline{E}_{y}^{\omega}$ field component is formally equivalent to an additional background permittivity $\Delta \epsilon_{\mathrm{r}}=\epsilon_{0}^{-1} \underline{P}_{y}^{\omega} / \underline{E}_{y}^{\omega}$, where $\underline{P}_{y}^{\omega}$ contains the polarization contribution of the quantum systems in frequency domain. In the following, we assume that the device operates in a single transverse mode with the magnetic field distribution $\underline{H}_{x}^{\mathrm{t}}$, possibly the fundamental mode. Using the similarity of Eq. 81 to the Schrödinger equation in quantum mechanics, we can apply perturbation theory in an analogous matter ${ }^{182}$ To first order, $\underline{H}_{x}^{\mathrm{t}}$ remains unchanged, and for the eigenvalue $\underline{\beta}^{2}$ we obtain the correction

$$
\Delta \underline{\beta}^{2}=\frac{\omega^{2}}{\epsilon_{0} \underline{E}_{y}^{\omega} c^{2}} \frac{\iint_{-\infty}^{\infty}\left|\underline{H}_{x}^{\mathrm{t}}\right|^{2} \underline{P}_{y}^{\omega} \mathrm{d} x \mathrm{~d} y}{\iint_{-\infty}^{\infty}\left|\underline{H}_{x}^{\mathrm{t}}\right|^{2} \mathrm{~d} x \mathrm{~d} y} .
$$

For completeness, we also include integration over the $y$ coordinate in Eq. 86 since the $y$ dependence of $\underline{H}_{x}^{\mathrm{t}}$ may be reintroduced based on above mentioned effective refractive index method. It should be mentioned that if $\underline{\epsilon}_{\mathrm{r}}$ in Eq. (81) has a non-vanishing imaginary part, the eigenvalue problem is non-Hermitian and strictly speaking, a biorthogonal basis set must be used.183 In this case, Eq. 86 serves as an approximation to the exact perturbation term. Furthermore, it is practical to split the unperturbed propagation constant $\beta$ into a real and an imaginary part, $\beta=\beta+\mathrm{i} \beta^{\prime}$. Here, $\beta^{\prime}$ is related to the power loss coefficient $a$ by $\beta^{\prime}=\operatorname{sgn}(\beta) a / 2$, with the sign function sgn. Assuming $|\beta| \gg\left|\beta^{\prime}\right|$, we can write $\underline{\beta}^{2} \approx \beta^{2}+\mathrm{i}|\beta| a+\Delta \underline{\beta}^{2}$. Introducing the effective waveguide refractive index $n_{\text {eff }}(\omega)$ defined by $\beta=\operatorname{sgn}(\beta) \omega n_{\text {eff }} / c$, we then obtain

$$
\begin{aligned}
\partial_{z} \underline{H}_{x}^{\omega}= & -\mathrm{i} \omega \epsilon_{0} n_{\text {eff }}^{2} \underline{E}_{y}^{\omega}+\epsilon_{0} c n_{\text {eff }} a \underline{E}_{y}^{\omega} \\
& -i \omega \frac{\iint_{-\infty}^{\infty}\left|\underline{H}_{x}^{\mathrm{t}}\right|^{2} \underline{P}_{y}^{\omega} \mathrm{d} x \mathrm{~d} y}{\iint_{-\infty}^{\infty}\left|\underline{H}_{x}^{\mathrm{t}}\right|^{2} \mathrm{~d} x \mathrm{~d} y} .
\end{aligned}
$$

a. Field Confinement Factor Equations (84) and (87) effectively reduce the complexity of the propagation problem from three spatial dimensions to a single coordinate $z$. However, for computing the integral in the polarization term of Eq. (87), $\underline{P}_{y}^{\omega}$ must be obtained by solving the Bloch equations in the whole device volume, using the full spatial field dependence given by Eq. 76. This greatly impedes the numerical efficiency of the one-dimensional propagation model. As indicated in Fig. 8(a), frequently the transverse field distribution does not vary significantly across the quantum nanostructure, e.g., because the nanostructure covers only part of the waveguide cross section, preferably at the position of maximum intensity. Consequently, also $\underline{P}_{y}^{\omega}$ is approximately constant over the quantum system cross section and can be taken out of the integral in Eq. (86), which can then be written as $\Gamma \underline{P}_{y}^{\omega}$. Here, $\Gamma$ denotes the field confinement factor, which gives the overlap of the quantum nanostructure with the mode profile and is thus also referred to as overlap factor. With Eq. (84), $\Gamma$ can be written as

$$
\Gamma=\frac{\iint_{A_{\mathrm{q}}}\left|\underline{H}_{x}^{\mathrm{t}}\right|^{2} \mathrm{~d} x \mathrm{~d} y}{\iint_{-\infty}^{\infty}\left|\underline{H}_{x}^{\mathrm{t}}\right|^{2} \mathrm{~d} x \mathrm{~d} y}=\frac{\iint_{A_{\mathrm{q}}}\left|\underline{E}_{y}^{\mathrm{t}}\right|^{2} \mathrm{~d} x \mathrm{~d} y}{\iint_{-\infty}^{\infty}\left|\underline{E}_{y}^{\mathrm{t}}\right|^{2} \mathrm{~d} x \mathrm{~d} y} .
$$

Here, the enumerator contains an integration over the cross section area $A_{\mathrm{q}}$ of the active region formed by the quantum systems. The intensity distribution in the waveguide is given by the time-averaged magnitude of the $z$ component of the Poynting vector, which is with Eq. 84 obtained as

$$
I=\left|\left\langle S_{z}\right\rangle\right|=\left|\Re\left\{\underline{E}_{y}^{\omega}\left(\underline{H}_{x}^{\omega}\right)^{*}\right\}\right| / 2=\epsilon_{0} c n_{\mathrm{eff}}\left|\underline{E}_{y}^{\omega}\right|^{2} / 2 .
$$




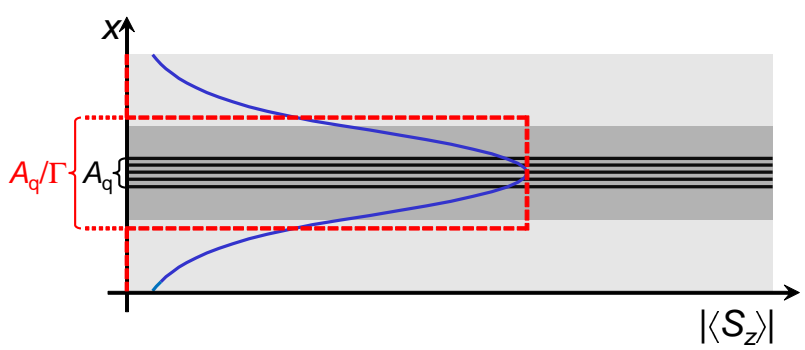

FIG. 9. Illustration of the transverse electric mode profile of the waveguiding structure in Fig.8. The solid curve shows the mode profile $\left|\underline{E}_{y}^{\mathrm{t}}\right|^{2}$, and the dashed curve represents the equivalent rectangular mode profile.

With Eqs. (84) and 88), we then arrive at the usual definition 184

$$
\Gamma=\frac{\iint_{A_{\mathrm{q}}}\left|\left\langle S_{z}\right\rangle\right| \mathrm{d} x \mathrm{~d} y}{\iint_{-\infty}^{\infty}\left|\left\langle S_{z}\right\rangle\right| \mathrm{d} x \mathrm{~d} y} .
$$

The meaning of $\Gamma$ is visualized in Fig.9. We can represent the field confinement factor as $\Gamma=A_{\mathrm{q}} / A_{\text {eff }}$ where $A_{\text {eff }}$ is the area covered by an equivalent mode which conserves $\iint_{-\infty}^{\infty}\left|\left\langle S_{z}\right\rangle\right| \mathrm{d} x \mathrm{~d} y$, but has a rectangular intensity distribution with $\left|\left\langle S_{z}\right\rangle\right|$ fixed to the value in the quantum nanostructure. Thus, the optical power can with Eq. 89 . be written as

$$
P=[I]_{\mathrm{q}} A_{\text {eff }}=\left|\left[\underline{E}_{y}^{\omega}\right]_{\mathrm{q}}\right|^{2} A_{\mathrm{eff}} \epsilon_{0} c n_{\mathrm{eff}} / 2,
$$

where $[I]_{\mathrm{q}}$ and $\left[\underline{E}_{y}^{\omega}\right]_{\mathrm{q}}$ refer to the values of $I$ and $\underline{E}_{y}^{\omega}$ in the quantum nanostructure.

b. One-Dimensional Maxwell Equations In the following, we regard the $\underline{E}_{y}^{\omega}$ and $\underline{H}_{x}^{\omega}$ fields at a frequency $\omega$ as spectral components of time dependent fields $E_{y}$ and $H_{x}$, and transform Eq. (87) into time domain. For convenience, we do not consider the $\omega$ dependence of $\underline{E}_{y}^{\mathrm{t}}$ and $\underline{H}_{x}^{\mathrm{t}}$ in Eq. 88 , but rather evaluate $\Gamma$ at the center frequency $\omega_{\mathrm{c}}$ of the optical field. Furthermore, to obtain a form compatible with Eq. $82 \mathrm{~b}$ ), we divide $n_{\text {eff }}^{2}(\omega)$ into a constant part, e.g., the value $n_{\text {eff }}^{2}\left(\omega_{c}\right)$ at $\omega=\omega_{\mathrm{c}}$, and a frequency dependent part $\Delta \epsilon_{\mathrm{eff}}=$ $n_{\text {eff }}^{2}(\omega)-n_{\text {eff }}^{2}\left(\omega_{\mathrm{c}}\right)$ which describes chromatic waveguide dispersion and gives rise to an extra polarization contribution. Considering that multiplications with $\omega$ in frequency domain correspond to operators $\mathrm{i} \partial_{t}$ in time domain, we obtain from Eqs. 84, (87) and 48

$$
\begin{aligned}
\partial_{z} E_{y} & =\mu_{0} \partial_{t} H_{x} \\
\partial_{z} H_{x} & =\epsilon_{0} n_{\mathrm{eff}}^{2}\left(\omega_{\mathrm{c}}\right) \partial_{t} E_{y}+\sigma\left(\mathrm{i} \partial_{t}\right) E_{y} \\
& +\Gamma n_{3 \mathrm{D}} \sum_{i, j} d_{y, j i} \partial_{t} \rho_{i j}+\epsilon_{0} \partial_{t}\left[\Delta \epsilon_{\mathrm{eff}}\left(\mathrm{i} \partial_{t}\right) E_{y}\right] .
\end{aligned}
$$

Here, the generally frequency dependent conductivity

$$
\sigma(\omega)=\epsilon_{0} c n_{\mathrm{eff}}(\omega) a(\omega)
$$

is often approximated by $\sigma=\sigma\left(\omega_{\mathrm{c}}\right)$. Obviously, $\Delta \epsilon_{\mathrm{eff}}$ and $\sigma$ must be even functions $f(-\omega)=f(\omega)$ to preserve the real-valued character of Eq. (92). Furthermore, causality requires that the real and imaginary parts of the complex permittivity defined in Eq. 78 fulfill the Kramers-Kronig relation, $\frac{185}{,}$ which is, strictly speaking, already violated when modeling a medium as a lossless, frequency independent dielectric with $\underline{\epsilon}_{\mathrm{r}}=\epsilon_{\mathrm{r}} \neq 1 ! 169$

Notably Eq. (92) does not explicitly depend on $x$ and $y$ anymore. Thus, it is practical to identify $H_{x}(z, t)$ and $E_{y}(z, t)$ with the field strengths at the transverse position of the nanostructure, because then $E_{y}$ can directly be used in Eq. (16) or (17) to evaluate $\partial_{t} \rho_{j i}(z, t)$. For completeness, we mention that the longitudinal magnetic field component $H_{z}$ can be obtained from $\nabla \mathbf{H}=0$, i.e., $\partial_{z} H_{z}=-\partial_{x} H_{x}$.

\section{Transverse Magnetic Mode}

For TM modes in slab waveguides, Eq. (77) yields with $\underline{H}_{x}^{\mathrm{t}}=0$ and $\partial_{y}=0$

$$
\underline{\epsilon}_{\mathrm{r}} \partial_{x}\left(\underline{\epsilon}_{\mathrm{r}}^{-1} \partial_{x} \underline{H}_{y}^{\mathrm{t}}\right)=\left(\underline{\beta}^{2}-\frac{\omega^{2}}{c^{2}} \underline{\epsilon}_{\mathrm{r}}\right) \underline{H}_{y}^{\mathrm{t}},
$$

and the boundary conditions are given by $\underline{H}_{y}^{\mathrm{t}}(x \rightarrow \pm \infty) \rightarrow 0$. The mutual dependence of the field components is given by Eq. (80), which yields with Eq. (76)

$$
\begin{aligned}
& \mathrm{i} \omega \epsilon_{0} \underline{\epsilon}_{\mathrm{r}} \underline{E}_{x}^{\omega}=\partial_{z} \underline{H}_{y}^{\omega}=\mathrm{i} \beta \underline{H}_{y}^{\omega}, \\
& \mathrm{i} \omega \epsilon_{0} \underline{\epsilon}_{\mathrm{r}} \underline{E}_{z}^{\omega}=-\partial_{x} \underline{H}_{y}^{\omega} .
\end{aligned}
$$

From Eq. 44a), we furthermore obtain with Eq. 76

$$
\partial_{z} \underline{E}_{x}^{\omega}=\mathrm{i} \omega \mu_{0} \underline{H}_{y}^{\omega}+\partial_{x} \underline{E}_{z}^{\omega} .
$$

Using Eq. 96 to eliminate $\underline{E}_{z}^{\omega}$ in Eq. 97 yields with Eq. 949

$$
\partial_{z} \underline{E}_{x}^{\omega}=\frac{\mathrm{i}}{\omega \epsilon_{0} \underline{\epsilon}_{\mathrm{r}}} \underline{\beta}^{2} \underline{H}_{y}^{\omega} .
$$

In Fig.10, the fundamental TM mode of a QCL waveguide structure is shown. The magnetic field distribution $\underline{H}_{y}^{\mathrm{t}}$ is continuous, while $\underline{E}_{x}^{\mathrm{t}}$ exhibits jumps at interfaces of layers with different $\underline{\epsilon}_{\mathrm{r}}$, as can also be seen from Eq. (95).

In the following, we treat the background refractive index $n_{0}$ of the host material for the quantum systems as a real constant, assuming that the main frequency dependence and gain/loss in the nanostructure is provided by the quantum systems rather than the host material. Furthermore, although for example in a quantum well structure the barrier and well materials will have different refractive indices as indicated in Figs. 8(b) and 9, the nanostructured region can still be approximately described by a single effective $\epsilon_{\mathrm{r}}$ since the individual layers are too thin to be resolved by the optical field. For TM modes, the effective permittivity is 


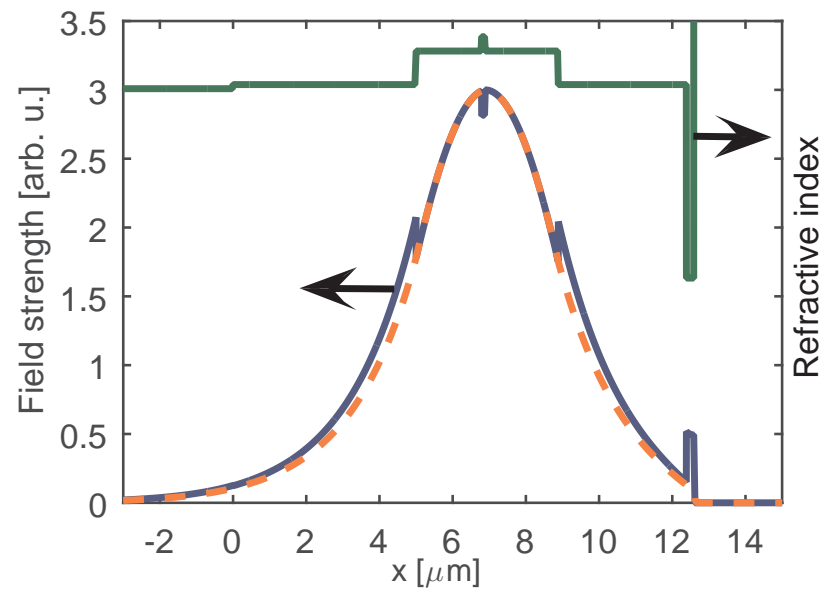

FIG. 10. Fundamental mode of a QCL waveguide structure at $28 \mathrm{THz} \cdot \frac{186}{186}$ The solid and dashed curves show $\left|\underline{E}_{x}^{\mathrm{t}}\right|$ and $\left|\underline{H}_{y}^{\mathrm{t}}\right|$, respectively. Furthermore, the refractive index profile, i.e., $\Re\left\{\underline{\epsilon}_{\mathrm{r}}^{1 / 2}(x)\right\}$, is displayed.

then obtained as the harmonic mean of the individual permittivity values, i.e., we obtain in the host material $n_{0}^{-2}=\left(\Delta_{1} \epsilon_{\mathrm{r}, 1}^{-1}+\Delta_{2} \epsilon_{\mathrm{r}, 2}^{-1}\right) /\left(\Delta_{1}+\Delta_{2}\right)$ where $\Delta_{i}$ and $\epsilon_{\mathrm{r}, i}$ denote the total thicknesses and permittivities of the regions made from material $i=1,2$ ! 187188

Generally, the treatment of TM modes is known to be more complex than for TE modes ${ }^{184}$ Specifically, in contrast to the TE case the derivation of one-dimensional Maxwell-type equations for the transverse field components is not as straightforward as in Section VA 1. This can for example be seen from Eq. (97), which only assumes a Maxwell-type form in analogy to Eq. (92a) if $\partial_{x} \underline{E}_{z}^{\omega}$ can be neglected. Similarly as in Section V A 1, we assume that the fields are approximately constant over the transverse cross section of the nanostructured region, and identify in the following $\underline{E}_{x}^{\omega}$ and $\underline{H}_{y}^{\omega}$ with the field strengths at the transverse position of the nanostructure. With $\underline{\epsilon}_{\mathrm{r}}=n_{0}^{2}$, we obtain from Eq. 98

$$
\partial_{z} \underline{E}_{x}^{\omega}=\mathrm{i} \omega \mu_{0} \frac{n_{\mathrm{eff}}^{2}\left(\omega_{\mathrm{c}}\right)}{n_{0}^{2}} \underline{H}_{y}^{\omega},
$$

where we have assumed that $\left|\beta^{\prime}\right| \ll|\beta|$ and approximated $\beta^{2} \approx \beta^{2}=n_{\text {eff }}^{2} \omega^{2} / c^{2}$ as in Section V A 1 , with the effective waveguide refractive index $n_{\text {eff }}$. Furthermore, we have neglected the frequency dependence of $n_{\text {eff }}$, evaluating it at the center frequency $\omega=\omega_{\mathrm{c}}$ of the optical field, so as to formally obtain a Maxwell-type equation with a frequency independent effective relative permeability $\mu_{\text {eff }}=n_{\text {eff }}^{2}\left(\omega_{\mathrm{c}}\right) / n_{0}^{2}$. In order to complete our model, Eq. (99) must be complemented by a second Maxwelltype equation with a form similar to Eq. 85 in frequency domain, i.e., Eq. 92b in time domain. Importantly, this equation has to include the losses and frequency dependence omitted in Eq. (99), so that the correct field propagation dynamics is obtained. Specifically, from Eq. (76) we obtain the field propagation equations in frequency domain $\partial_{z}^{2} \underline{E}_{x}^{\omega}=-\underline{\beta}^{2} \underline{E}_{x}^{\omega}, \partial_{z}^{2} \underline{H}_{y}^{\omega}=-\underline{\beta}^{2} \underline{H}_{y}^{\omega}$. This requires that

$$
\partial_{z} \underline{H}_{y}^{\omega}=\mathrm{i} \frac{n_{0}^{2}}{\omega \mu_{0} n_{\mathrm{eff}}^{2}\left(\omega_{\mathrm{c}}\right)} \underline{\beta}^{2} \underline{E}_{x}^{\omega},
$$

as can be verified by differentiating Eq. 99 with respect to $z$ and eliminating $\underline{H}_{y}^{\omega}$ with Eq. 100 , or alternatively eliminating $\underline{E}_{x}^{\omega}$ in an analogous way.

As in Section V A 1, the polarization due to the quantum systems is again perturbatively included in terms of a change $\Delta \beta^{2}$ to $\beta^{2}$. To this end, we re-derive Eq. 94 from Maxwell's equations, Eq. (44), but now keep the polarization contribution, which yields on the left side of Eq. (94) the perturbation term $\beta \omega \underline{P}_{x}^{\omega}$. Here, $\underline{P}_{x}^{\omega}$ is the $x$ component of $\mathbf{P}_{\mathrm{q}}$ in frequency domain, while a possible additional $z$ component has been neglected. With Eq. 95 and $\beta^{2} \approx \beta^{2}=n_{\text {eff }}^{2} \omega^{2} / c^{2}$, the perturbation term can then be written as $\beta \omega \underline{P}_{x}^{\omega} \approx \Delta L \underline{H}_{y}^{\mathrm{t}}$ with

$$
\Delta L \approx \frac{\omega^{2}}{c^{2}} \frac{n_{\mathrm{eff}}^{2}}{n_{0}^{2}} \frac{\underline{P}_{x}^{\omega}}{\epsilon_{0} \underline{E}_{x}^{\omega}}
$$

and Eq. 940 becomes $(\hat{L}+\Delta L) \underline{H}_{y}^{\mathrm{t}}=\underline{\beta}^{2} \underline{H}_{y}^{\mathrm{t}}$ with $\hat{L}=$ $\underline{\epsilon}_{\mathrm{r}} \partial_{x} \underline{\epsilon}_{\mathrm{r}}^{-1} \partial_{x}+\omega^{2} c^{-2} \underline{\epsilon}_{\mathrm{r}}$. Similarly as in Section V A 1 . we assume that the device operates in a given transverse mode with propagation constant $\beta$, and use that first order perturbation theory does not affect the corresponding eigenfunction $\underline{H}_{y}^{\mathrm{t}}$. Since $\hat{L}$ is non-Hermitian, a biorthogonal basis set must be used, and the change of $\underline{\beta}^{2}$ is given by $\Delta \underline{\beta}^{2}=\langle\tilde{\phi}|\Delta L| \phi\rangle /\langle\tilde{\phi} \mid \phi\rangle$ ! ${ }^{183}$ Here $\phi=$ $H_{y}^{\mathrm{t}}$, while $\tilde{\phi}$ denotes the corresponding eigenfunction of the adjoint problem $\hat{L}^{\dagger} \tilde{\phi}(x)=\left(\underline{\beta}^{2}\right)^{*} \tilde{\phi}(x)$, with $\hat{L}^{\dagger}=$ $\partial_{x}\left(\underline{\epsilon}_{\mathrm{r}}^{-1}\right)^{*} \partial_{x}\left(\underline{\epsilon}_{\mathrm{r}}^{*} \ldots\right)+\omega^{2} c^{-2} \underline{\epsilon}_{\mathrm{r}}^{*}$ and $\tilde{\phi}(x \rightarrow \pm \infty) \rightarrow 0$. As can be seen by inserting Eq. (95) into Eq. (94), $\tilde{\phi}$ simply corresponds to the conjugate complex electric field distribution of the mode $\left(\underline{E}_{x}^{\mathrm{t}}\right)^{*}$, and in analogy to Section V A 1 a we then obtain the field confinement factor $\Gamma=\iint_{A_{\mathrm{q}}} \underline{E}_{x}^{\mathrm{t}} \underline{H}_{y}^{\mathrm{t}} \mathrm{d} x \mathrm{~d} y / \iint_{-\infty}^{\infty} \underline{E}_{x}^{\mathrm{t}} \underline{H}_{y}^{\mathrm{t}} \mathrm{d} x \mathrm{~d} y$. Generally, this expression is complex, but for real $\underline{\epsilon}_{\mathrm{r}}$ it coincides with the previous result Eq. (90). Thus, the expression for the confinement factor given in Eq. (90) corresponds to the perturbative expression for both TE and TM modes in the case of real $\underline{\epsilon}_{\mathrm{r}}$, and is commonly also used for complex $\underline{\epsilon}_{\mathrm{r}}$ where it can be seen as a real-valued approximation to the perturbative result. Similarly as in Section VA 1, we insert $\beta^{2} \approx \beta^{2}+\mathrm{i}|\beta| a+\Delta \beta^{2}$ with $\beta(\omega)=\operatorname{sgn}(\beta) \omega n_{\text {eff }}(\omega) / \bar{c}$ into Eq. 100, which yields

$$
\begin{aligned}
\partial_{z} \underline{H}_{y}^{\omega}= & \mathrm{i} \omega \frac{\epsilon_{0} n_{0}^{2}}{n_{\mathrm{eff}}^{2}\left(\omega_{\mathrm{c}}\right)} n_{\mathrm{eff}}^{2}(\omega) \underline{E}_{x}^{\omega}-\frac{\epsilon_{0} n_{0}^{2}}{n_{\mathrm{eff}}^{2}\left(\omega_{\mathrm{c}}\right)} c n_{\mathrm{eff}}(\omega) a \underline{E}_{x}^{\omega} \\
& +\mathrm{i} \omega \Gamma \underline{P}_{x}^{\omega} .
\end{aligned}
$$

Here, we have neglected a possible frequency dependence of $n_{\text {eff }}$ and $\Gamma$ in the last term. Obviously, our two derived Maxwell-type equations, Eqs. (99) and (102), have the same form as Eqs. (84) and (87) for TE modes, as can 
be seen by substituting $\underline{H}_{x}^{\omega} \rightarrow-\underline{H}_{y}^{\omega}, \underline{E}_{y}^{\omega} \rightarrow \underline{E}_{x}^{\omega}, \epsilon_{0} \rightarrow$ $\epsilon_{0} n_{0}^{2} / n_{\text {eff }}^{2}\left(\omega_{\mathrm{c}}\right), \mu_{0} \rightarrow \mu_{0} n_{\text {eff }}^{2}\left(\omega_{\mathrm{c}}\right) / n_{0}^{2}$. Thus, the Maxwelltype equations in time domain can be obtained in the same way as Eq. (92), yielding

$$
\begin{aligned}
\partial_{z} E_{x} & =-\mu_{0} \frac{n_{\mathrm{eff}}^{2}\left(\omega_{\mathrm{c}}\right)}{n_{0}^{2}} \partial_{t} H_{y} \\
\partial_{z} H_{y} & =-\epsilon_{0} n_{0}^{2} \partial_{t} E_{x}-\sigma\left(\mathrm{i} \partial_{t}\right) E_{x} \\
& -\Gamma n_{3 \mathrm{D}} \sum_{i, j} d_{x, j i} \partial_{t} \rho_{i j}-\epsilon_{0} \partial_{t}\left[\Delta \epsilon_{\mathrm{eff}}\left(\mathrm{i} \partial_{t}\right) E_{x}\right]
\end{aligned}
$$

where the generally frequency dependent conductivity

$$
\sigma(\omega)=\epsilon_{0} n_{0}^{2} c n_{\mathrm{eff}}(\omega) a(\omega) / n_{\mathrm{eff}}^{2}\left(\omega_{\mathrm{c}}\right)
$$

is often approximated by $\sigma=\sigma\left(\omega_{\mathrm{c}}\right)$. Furthermore, in the last term describing chromatic waveguide dispersion, we now have $\Delta \epsilon_{\text {eff }}=n_{0}^{2} n_{\text {eff }}^{2}(\omega) / n_{\text {eff }}^{2}\left(\omega_{\mathrm{c}}\right)-n_{0}^{2}$. As discussed below Eq. (93), certain conditions apply to $\Delta \epsilon_{\text {eff }}$ and $\sigma$. In particular, they must be even functions $f(-\omega)=f(\omega)$ to preserve the real-valued character of Eq. (103). Identifying $E_{x}(z, t)$ and $H_{y}(z, t)$ with the field strengths at the transverse position of the nanostructure, $E_{x}$ can directly be used in Eq. (16) or (17) to evaluate $\partial_{t} \rho_{i j}(z, t)$. As stated above, Eq. (103) has been constructed to assume the form of one-dimensional Maxwell equations and to yield the correct propagation behavior for $E_{x}$ and $H_{y}$. On the other hand, the relation between $E_{x}$ and $H_{y}$, given by Eq. (95) for $\underline{P}_{x}^{\omega}=0$, is in Eq. (103) for the general case of waveguide loss and dispersion only approximately fulfilled.

\section{B. Slowly Varying Amplitude Approximation}

As in Section $\mathrm{VA}$, we assume a waveguiding structure which is invariant in propagation direction $z$, and now employ the slowly varying amplitude approximation. The guided mode solutions at a given frequency $\omega$ are characterized by the propagation constant and a $z$ independent transverse field distribution $F(x, y)$. Here $F$ is induced by the refractive index profile $\Delta_{n}(x, y)$, while the polarization of the quantum systems and other nonlinear effects are assumed to act as perturbations which do not significantly affect the transverse field distribution. 181

We start from Eq. 60) and introduce the slowly varying field envelopes by inserting Eq. (61), where we however replace $k_{\mathrm{c}}$ by the propagation constant of the guided mode $\beta_{0}=\beta\left(\omega_{\mathrm{c}}\right)$. In the following, it is advantageous to switch to the spectral domain, where the slowly varying envelopes depend on the frequency variable $\Delta_{\omega}=\omega-\omega_{\mathrm{c}}$, corresponding to the frequency offset from $\omega_{\mathrm{c}}$. Neglecting higher order derivatives of $t$ and $z$ in the spirit of the SVAA and the paraxial approximation as described in Section IVB, and considering that time derivatives of the envelopes are replaced by multiplications with $-\mathrm{i} \Delta_{\omega}$ in frequency domain, we obtain

$$
-2 \mathrm{i} \beta_{0} \partial_{z} \underline{\mathbf{E}}+\beta_{0}^{2} \underline{\mathbf{E}}=\nabla_{\mathrm{T}}^{2} \underline{\mathbf{E}}+\frac{\omega^{2}}{c^{2}} \underline{n}^{2} \underline{\mathbf{E}}+\omega_{\mathrm{c}}^{2} \mu_{0} \underline{\mathbf{P}} .
$$

For the term $\omega^{2} c^{-2} \underline{n}^{2} \underline{\mathbf{E}}$, we have retained the full frequency dependence of the complex refractive index $\underline{n}(x, y, \omega)$ to include chromatic dispersion, as described further below. More specifically, $\underline{n}^{2}=$ $n_{0}^{2}\left(1-2 \Delta_{n}\right)+\mathrm{i} \sigma /\left(\omega \epsilon_{0}\right)$ contains the refractive index profile via $\Delta_{n}(x, y)$ and losses via the conductivity $\sigma$, where $n_{0}, \Delta_{n}$ and $\sigma$ may be treated as frequency dependent. Assuming a guided mode solution, we can use the separation ansatz

$$
\underline{\mathbf{E}}(\mathbf{x}, t)=\mathbf{e} \underline{E}(z, t) F(x, y),
$$

with the polarization direction of the electric field $\mathbf{e}$ and modal distribution $F$. Inserting Eq. 106 into Eq. 105, multiplying by e and introducing the separation constant $\underline{\beta}^{2}$, the right side of the resulting equation becomes

$$
\left(\underline{\beta}^{2}-\frac{\omega^{2}}{c^{2}} \underline{n}^{2}\right) F=\nabla_{\mathrm{T}}^{2} F
$$

which does not depend on the propagation coordinate $z$. As in Section VA 1, $\underline{\mathbf{P}}$ is subsequently included based on first order perturbation theory 181 Equation (107), together with the boundary condition that $F \rightarrow 0$ for $x^{2}+y^{2} \rightarrow \infty$, constitutes an eigenvalue equation for $F$ with complex eigenvalues $\beta^{2}$, featuring multiple eigensolutions which correspon $\bar{d}$ to the different transverse waveguide modes. In the following, we assume that the device operates in a single transverse mode, possibly the fundamental mode. As in Section VA 1, we split the complex propagation constant $\beta$ into a real and an imaginary part $\beta=\beta+\operatorname{sgn}(\beta)$ i $a / 2$ with power loss coefficient $a$, and assume that $|a| \ll|\beta|$. Including $\underline{\mathbf{P}}$ in first order perturbation theory in analogy to Section V A 1 does not alter $F$, but yields a modified propagation constant $\beta+\Delta \beta$ with 181

$$
\Delta \beta=\frac{\omega_{\mathrm{c}}^{2}}{2 c^{2} \beta_{0}} \frac{\iint_{-\infty}^{\infty} \Delta \epsilon_{\mathrm{r}}|F|^{2} \mathrm{~d} x \mathrm{~d} y}{\iint_{-\infty}^{\infty}|F|^{2} \mathrm{~d} x \mathrm{~d} y} \approx \frac{\omega_{\mathrm{c}}^{2}}{2 c^{2} \beta_{0}} \Gamma \Delta \epsilon_{\mathrm{r}},
$$

where $\Delta \epsilon_{\mathrm{r}}=\mathbf{e} \underline{\mathbf{P}} /\left(\epsilon_{0} \underline{E}\right)$ and $\Gamma=$ $\iint_{A_{\mathrm{q}}}|F|^{2} \mathrm{~d} x \mathrm{~d} y / \iint_{-\infty}^{\infty}|F|^{2} \mathrm{~d} x \mathrm{~d} y$ in agreement with Eq. 88. Here we have evaluated $\Delta \beta$ at the carrier frequency $\omega_{\mathrm{c}}$, in accordance with the SVAA. For a realistic description of guided mode propagation, the frequency dependence of $\beta$ itself should however be retained, giving rise to chromatic dispersion!181 This effect is commonly described in terms of a Taylor series, $\beta\left(\Delta_{\omega}\right)=\sum_{n}\left(\beta_{n} / n !\right) \Delta_{\omega}^{n}$ with $\beta_{n}=\left[\mathrm{d}_{\omega}^{n} \beta\right]_{\omega=\omega_{\mathrm{c}}}$. While frequency dependent waveguide loss can be included in a similar manner by an $\omega$ dependent coefficient $a$, we ignore this effect since usually the spectral gain or loss profile is dominated by the contribution of the 
quantum systems, contained in $\underline{\mathbf{P}}$. Furthermore assuming that $|\Delta \beta| \ll|\beta|$ and $\beta \approx \beta_{0}$, we can approximate $\beta^{2}-\beta_{0}^{2} \approx 2 \beta_{0}\left(\beta-\beta_{0}\right)+\mathrm{i}\left|\beta_{0}\right| a+2 \beta_{0} \Delta \beta$. With this result and Eq. (108), the separation ansatz yields for the left-hand side of Eq. 105 in time domain

$$
\begin{aligned}
\frac{1}{v_{\mathrm{g}}} \partial_{t} \underline{E}+\partial_{z} \underline{E}= & \mathrm{i} \sum_{n \geq 2} \frac{\beta_{n}}{n !}\left(\mathrm{i} \partial_{t}\right)^{n} \underline{E}-\operatorname{sgn}\left(\beta_{0}\right) \frac{a}{2} \underline{E} \\
& +\mathrm{i} \frac{\omega_{\mathrm{c}}^{2}}{2 \epsilon_{0} \beta_{0} c^{2}} \Gamma \underline{\mathbf{E}},
\end{aligned}
$$

where $v_{\mathrm{g}}=\beta_{1}^{-1}$ denotes the group velocity at $\omega_{\mathrm{c}}$.

The guided field solution is characterized by a linearly polarized field distribution, with the electric field pointing in direction e as reflected by the ansatz for the electric field, Eq. 106]!169|181 The corresponding modes are transverse electromagnetic, i.e., with transverse, perpendicular electric and magnetic fields. Notably, this approach always yields two degenerate modes, orthogonally polarized in transverse $x$ and $y$ directions. In reality, this applies for example to an ideal, cylindrically symmetric single-mode fiber, while irregularities such as random variations in the core shape already break the degeneracy. Within the assumptions of weak waveguiding, the optical power is given by the corresponding expression for the TE mode, Eq. (91).

Above approach is commonly used to model coherent propagation effects in optical fibers like self-induced transparency, where the dopants, such as erbium ions, take the role of the quantum systems modeled by the Bloch equations, and the host material is for example glass $\frac{189}{192}$ Here, in addition to the refractive index profile, fiber loss and chromatic dispersion, also other effects related to the host material are commonly considered. This in particular includes optical nonlinearity due to an intensity dependent refractive index of the host material, which induces an intensity dependent phase shift of the optical field and is thus referred to as self-phase modulation. This effect can be included in Eq. (105) by substituting $\underline{n}^{2}$ with $\left(\underline{n}+n_{2}|\underline{\mathbf{E}}|^{2}\right)^{2} \approx \underline{n}^{2}+2 n_{0} n_{2}|\underline{E} F|^{2} \underline{\underline{181}}$ Treating the nonlinear component as a perturbation, we can again use Eq. 108 with $\Delta \epsilon_{\mathrm{r}}=2 n_{0} n_{2}|\underline{E} F|^{2}$ and include this effect in a similar manner as discussed above. With Eqs. 109), 64) and (88), we finally obtain the propagation equation

$$
\begin{aligned}
\frac{1}{v_{\mathrm{g}}} \partial_{t} \underline{E}+\partial_{z} \underline{E} & =\mathrm{i} \sum_{n \geq 2} \frac{\beta_{n}}{n !}\left(\mathrm{i} \partial_{t}\right)^{n} \underline{E}-\operatorname{sgn}\left(\beta_{0}\right) \frac{a}{2} \underline{E} \\
& +\mathrm{i} \gamma|\underline{E}|^{2} \underline{E}+\mathrm{i} \frac{n_{3 \mathrm{D}} \omega_{\mathrm{c}}^{2}}{\epsilon_{0} \beta_{0} c^{2}} \Gamma \sum_{\omega_{i j}>0} d_{j i} \eta_{i j},
\end{aligned}
$$

with the self-phase modulation coefficient

$$
\gamma=\frac{n_{0} n_{2} \omega_{\mathrm{c}}^{2}}{\beta_{0} c^{2}} \frac{\iint_{-\infty}^{\infty}|F|^{4} \mathrm{~d} x \mathrm{~d} y}{\iint_{-\infty}^{\infty}|F|^{2} \mathrm{~d} x \mathrm{~d} y} .
$$

We note that in Eq. 1111, the nonlinearity is assumed to extend over the whole fiber cross section since both the core and cladding typically consist of the same host material. The MB equations are then obtained by coupling Eq. 110 to the Bloch equations in RWA, Eq. 43. Here it is practical to normalize $F$ in Eq. (106) so that $F=1$ at the transverse position of the dopants acting as quantum systems; then the field in Eq. 43 is directly given by $\underline{\mathbf{E}}=\mathbf{e} \underline{E}(z, t)$.

Typically, the MB equations are stepped in time to obtain the temporal evolution of the optical field in a given geometry. For the case of unidirectional propagation along a fiber where the input at $z=0$ is a given time-limited optical waveform such as a pulse, it is more practical to propagate the field in $z$ direction. It is then convenient to introduce the retarded time variable $\tau=t-z / v_{\mathrm{g}}$, which is defined with respect to a time frame which co-propagates with the waveform. Denoting the position variable in the new coordinate system as $\zeta=z$, we then obtain the partial derivatives $\partial_{z}=\partial_{\zeta}-\left(1 / v_{\mathrm{g}}\right) \partial_{\tau}$, $\partial_{t}=\partial_{\tau}$. Thus, Eq. 110 becomes

$$
\begin{aligned}
\partial_{z} \underline{E}= & \mathrm{i} \sum_{n \geq 2} \frac{\beta_{n}}{n !}\left(\mathrm{i} \partial_{\tau}\right)^{n} \underline{E}-\operatorname{sgn}\left(\beta_{0}\right) \frac{a}{2} \underline{E}+\mathrm{i} \gamma|\underline{E}|^{2} \underline{E} \\
& +\mathrm{i} \frac{n_{3 \mathrm{D}} \omega_{\mathrm{c}}^{2}}{\epsilon_{0} \beta_{0} c^{2}} \Gamma \sum_{\omega_{i j}>0} d_{j i} \eta_{i j}
\end{aligned}
$$

where we have resubstituted $\zeta$ with $z$. For very short pulses with durations of only a few optical cycles, additional corrections might have to be included on the right side of Eq. 112. In particular, this includes the self-steepening term $-\gamma \omega_{\mathrm{c}}^{-1} \partial_{\tau}\left(|\underline{E}|^{2} \underline{E}\right)^{1811193}$ which is a higher order term dropped in the SVAA, and the Raman-induced frequency shift term $-\mathrm{i} \gamma T_{\mathrm{R}} \underline{E} \partial_{\tau}\left(|\underline{E}|^{2}\right)$ with Raman-response time $T_{\mathrm{R}}{ }^{181|194| 195}$ In Eqs. (110) and $(112)$, we have assumed that $d_{j i}$ and $\underline{E}$ are aligned in the same direction or, as is more realistic for an optical fiber, $d_{j i}$ are effective dipole moments which average over the different orientations of the dopant ions with respect to the field. Equation 1112 is solved together with the Bloch equations in RWA, Eq. 43, which are expressed in the retarded time frame simply by substituting $t$ with $\tau$ in the density matrix elements and derivative operators. The resulting equation system is sometimes also referred to as Hirota-Maxwell-Bloch system.196

\section{Fabry-Pérot Type Resonator}

For lasers, optical feedback has to be provided, which is in semiconductor lasers typically achieved by using a Fabry-Pérot type waveguide resonator. Here, the cleaved end facets provide natural reflection due to the refractive index jump between the semiconductor material and air. 


\section{Boundary Conditions at the End Facets}

In the Fabry-Pérot type resonator, the ansatz for the optical field, Eqs. 61a), is extended to include a forward and a backward propagating component, with amplitudes $\underline{E}_{+}$and $\underline{E}_{-}$, respectively. Furthermore assuming a guided mode solution as in Eq. (106), we obtain

$$
\begin{aligned}
E(z, t)= & \frac{1}{2}\left[\underline{E}_{+}(z, t) \exp \left(\mathrm{i} \beta_{0} z-\mathrm{i} \omega_{\mathrm{c}} t\right)\right. \\
& \left.+\underline{E_{-}}(z, t) \exp \left(-\mathrm{i} \beta_{0} z-\mathrm{i} \omega_{\mathrm{c}} t\right)+c . c .\right],
\end{aligned}
$$

where $\beta_{0}$ is the real part of the propagation constant at $\omega=\omega_{\mathrm{c}}$. With the (generally complex) field reflection coefficients $\underline{r}_{1}$ and $\underline{r}_{2}$ of the facets, assumed to be located at $z=0$ and $z=L$ where $L$ is the resonator length, we obtain

$$
\begin{aligned}
& \underline{E}_{+}(z=0, t)=\underline{r}_{1} \underline{E}_{-}(z=0, t), \\
& \underline{E}_{-}(z=L, t)=\underline{r}_{2} \underline{E}_{+}(z=L, t),
\end{aligned}
$$

where we have neglected a possible frequency dependence of $\underline{r}_{1,2}$.

For the full Maxwell equations, a decomposition of the field into a forward and a backward propagating component is not practical. Here, reflecting boundary conditions can in principle be implemented by position dependent parameters, e.g., by setting $n_{\mathrm{eff}}=1, \Delta \epsilon_{\mathrm{eff}}=0$, $\sigma=0$ and $d_{y, j i}=0$ in Eq. (92b) for $z<0$ and $z>L$ if we assume air outside of the resonator region and neglect modal effects. In this context, care has to be taken to suppress unwanted spurious reflections at the simulation domain boundaries, which can be achieved by implementing absorbing boundary conditions. However, this is not quite trivial, and various methods with different degrees of complexity have been developed! ${ }^{52} \mathrm{~A}$ simplified treatment, which works best for highly reflecting facets, is to use perfectly reflecting boundary conditions by setting the transverse electric field component at the facet positions to zero. The mirror loss, i.e., the decay of the optical field in the cavity due to outcoupling through the mirrors, can then be considered by a distributed power loss coefficient $a_{\mathrm{m}}$, which is is obtained from $\left|\underline{r}_{1} \underline{r}_{2}\right|^{2}=\exp \left(-2 a_{\mathrm{m}} L\right)$ as

$$
a_{\mathrm{m}}=-\ln \left(\left|\underline{r}_{1} \underline{r}_{2}\right|\right) / L .
$$

Using Eq. 93 or 104, $\sigma$ in Eq. 92b or 103b can then be determined from the total power loss coefficient $a=$ $a_{\mathrm{m}}+a_{\mathrm{w}}$, where $a_{\mathrm{w}}=2 \operatorname{sgn}(\Re\{\underline{\beta}\}) \Im\{\underline{\beta}\}$ denotes the waveguide loss.

a. Reflection Coefficient Using special reflective structures, such as reflection/antireflection coatings or distributed Bragg reflectors, $\underline{r}_{1,2}$ can be custom-tailored. In the following, we focus on the highly relevant case where the bare end facets are used as reflective elements. For sufficiently large transverse waveguide dimensions, Fresnel's formula for normal incidence can be used to es- timate the field reflection coefficient at the facet as

$$
\underline{r}=\frac{\underline{n}_{\mathrm{eff}}-1}{\underline{n}_{\mathrm{eff}}+1}=\frac{\underline{\beta}-k_{0}}{\underline{\beta}+k_{0}},
$$

with $\underline{n}_{\text {eff }}=\beta / k_{0}$ and $k_{0}=\omega / c$. While Eq. 116 is usually valid for weak waveguiding assumed in the derivation of Eqs. (60) and (110), modal effects can result in increased reflection at the facets $\frac{197}{}$ Various methods are available to compute the reflectance $R=|r|^{2}$ from the transverse mode profile! 198

For TE polarization, it is practical to decompose the waveguide mode, characterized by its complex propagation constant $\underline{\beta}$ and magnetic field distribution $\underline{H}_{x}^{\mathrm{t}}$ which can be computed from Eq. (81), into plane waves, using the Fourier transform

$$
\Phi_{x}\left(k_{x}\right)=\int_{-\infty}^{\infty} \underline{H}_{x}^{\mathrm{t}}(x) \exp \left(-\mathrm{i} k_{x} x\right) \mathrm{d} x .
$$

Then, a generalized version of Eq. 116 for tilted incidence is applied to each plane wave in order to calculate the reflection coefficient 198 The reflectance $R$, i.e., the ratio of the optical power reflected at the facet to the incident power, is obtained by integrating over all components, yielding

$$
R=\frac{1}{2 \pi}\left[\int_{-\infty}^{\infty}\left|\underline{H}_{x}^{\mathrm{t}}\right|^{2} \mathrm{~d} x\right]^{-1} \int_{-\infty}^{\infty}|\underline{\underline{\beta}-\kappa}|^{2}\left|\Phi_{x}\right|^{2} \mathrm{~d} k_{x}
$$

where $\kappa\left(k_{x}\right)=\sqrt{k_{0}^{2}-k_{x}^{2}}$ and the square root is chosen so that $\Im\{\kappa\}>0$. From Eq. (84), we see that Eq. (118) can also be evaluated by replacing $\underline{H}_{x}^{\mathrm{t}}$ with $\underline{E}_{y}^{\mathrm{t}}$ in Eqs. (118) and 117). Making the reasonable assumption that $\Re\{\underline{\beta}\}|\gg| \Im\{\underline{\beta}\} \mid$, we can approximately treat the reflection coefficient $r$ as real-valued. Furthermore assuming that the share of reflected power going into other waveguide modes is negligible, ${ }^{198}$ we obtain $r=R^{1 / 2}$.

The case of TM polarization is somewhat more complex and can be treated based on the boundary value method 198 Starting from the magnetic field distribution $\underline{H}_{y}^{\mathrm{t}}$ given by Eq. (94), we first evaluate the power transmittance $T$ through the facet, 119

$$
\begin{aligned}
T & =\frac{2}{\pi}|\underline{\beta}|^{2}\left[\int_{-\infty}^{\infty}\left|\underline{\epsilon}_{\mathrm{r}}\right|^{-2} \Re\left\{\underline{\beta}^{*} \underline{\epsilon}_{\mathrm{r}}\right\}\left|\underline{H}_{y}^{\mathrm{t}}\right|^{2} \mathrm{~d} x\right]^{-1} \\
& \times \int_{-k_{0}}^{k_{0}} \frac{\kappa\left|\Phi_{y}\right|^{2}\left|\Phi_{y}^{\prime}\right|^{2}}{\left|\kappa \Phi_{y}+\underline{\beta} \Phi_{y}^{\prime}\right|^{2}} \mathrm{~d} k_{x} .
\end{aligned}
$$

Here, $\Phi_{y}\left(k_{x}\right)$ denotes the Fourier transform, Eq. 117), of $\underline{H}_{y}^{\mathrm{t}}(x)$, and $\Phi_{y}^{\prime}\left(k_{x}\right)$ is the Fourier transform applied to the function $\underline{\epsilon}_{\mathrm{r}}^{-1}(x) \underline{H}_{y}^{\mathrm{t}}(x)$, with the complex relative permittivity profile of the slab waveguide structure $\underline{\epsilon}_{\mathrm{r}}(x)$. Again neglecting modal effects and assuming a real $r$, the reflection coefficient is then given by $r=(1-T)^{1 / 2}$. 


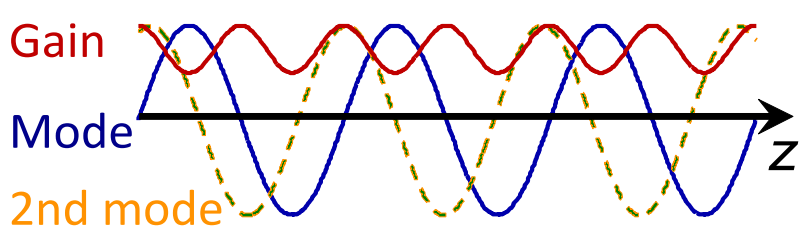

FIG. 11. Illustration of spatial hole burning in a Fabry-Pérot cavity.

Since the field distributions and $\beta$ in Eqs. (118) and (119) depend on $\omega$, this also applies to the obtained reflection coefficients. Usually, this frequency dependence is neglected in the formulation of the boundary conditions, and $r$ is taken at the center frequency $\omega_{c}$ of the optical field.

\section{Spatial Hole Burning}

In a Fabry-Pérot resonator, the reflection at the end facets gives rise to counterpropagating waves, which produce a standing wave pattern with a periodicity corresponding to the wavelength. At the field node positions, there is no interaction of the optical field with the quantum systems. This also implies that the population inversion and resulting optical gain, as provided by the quantum systems in the active region of a semiconductor laser, do not get saturated at those positions. Thus, other modes at slightly different frequencies which have their maxima close to these unsaturated regions can also start lasing. In Fig. 11, this effect is illustrated, which is referred to as (longitudinal) spatial hole burning (SHB). The resulting multimode lasing can be desired or undesired, depending on the envisaged application. For example, the broadening of the lasing spectrum is beneficial in applications such as the generation of frequency combs in QCLs, which are comb-like optical spectra used for precision metrology and sensing 97 On the other hand, spatial hole burning tends to introduce optical instabilities in form of irregular variations in the mode amplitudes and phases. $\frac{97 \mid 202}{}$ In a similar way as just discussed for the propagation direction, SHB can also occur along the transverse directions, and has been shown to affect the spatiotemporal dynamics especially in broad-area semiconductor lasers 203

The inversion grating is smoothed out by carrier diffusion processes, and SHB can even be neglected in a first approximation if diffusion is strong enough!118 Diffusion can be generically described by adding a term $\left.\partial_{t} \rho_{i i}\right|_{\text {diff }}=\nabla D_{i} \nabla \rho_{i i}$ to the Bloch equations, Eq. (16) or 17] 118 204 Here $D_{i}(\mathbf{x})$ is the diffusion coefficient associated with level $i$. In the following, we focus on longitudinal SHB. Furthermore assuming constant coefficients $D_{i}$, the diffusion term added to the Bloch equations, Eq. 116 or 17, becomes

$$
\left[\partial_{t} \rho_{i i}\right]_{\mathrm{diff}}=D_{i} \partial_{z}^{2} \rho_{i i}
$$

with zero-flux boundary conditions $\partial_{z} \rho_{i i}=0$ at the resonator ends. In a two-level description of bulk semiconductors, the levels $i$ correspond to the conduction and valence bands, and the diffusion process is largely mediated by carrier-phonon and carrier-carrier scattering between the $\mathbf{k}$ states in the bands. $\frac{118}{1 n}$ quantum wells, the levels correspond to the subbands formed by onedimensional carrier confinement, and the diffusion process is mediated by scattering between the $\mathbf{k}$ states in the subbands. ${ }^{205}$ In multi-quantum-dot structures, the SHB dynamics is often modeled by taking into account the carrier diffusion in the wetting layer, as well as carrier capture and escape processes to and from the quantum dots.206|207 Since these processes effectively reduce the diffusion length, SHB can have a strong effect, similarly as in intersubband devices like QCLs which typically feature a very fast gain recovery dynamics.208 On the other hand, the inversion grating is usually eliminated in interband bulk and quantum well lasers due to effective diffusion.207

a. Slowly Varying Amplitude Approximation As for Eq. (42), we assume that all transitions between pairs of states $i$ and $j$ with non-negligible coupling to the optical field are in near-resonance, $\left|\omega_{i j}\right| \approx \omega_{\mathrm{c}}$. For the corresponding off-diagonal density matrix elements, we now make the ansatz

$$
\begin{aligned}
\rho_{i j} & =\eta_{i j}^{+}(z, t) \exp \left[\operatorname{sgn}\left(\omega_{i j}\right) \mathrm{i}\left(\beta_{0} z-\omega_{\mathrm{c}} t\right)\right] \\
& +\eta_{i j}^{-}(z, t) \exp \left[\operatorname{sgn}\left(\omega_{i j}\right) \mathrm{i}\left(-\beta_{0} z-\omega_{\mathrm{c}} t\right)\right] .
\end{aligned}
$$

The periodicity of the inversion grating corresponds to that of the optical intensity, i.e., half the wavelength. Thus, for the populations we make the ansatz

$$
\begin{aligned}
\rho_{i i}= & \rho_{i i}^{0}(z, t)+\rho_{i i}^{+}(z, t) \exp \left(2 \mathrm{i} \beta_{0} z\right) \\
& +\rho_{i i}^{-}(z, t) \exp \left(-2 \mathrm{i} \beta_{0} z\right)
\end{aligned}
$$

where $\rho_{i i}^{+}=\left(\rho_{i i}^{-}\right)^{*}$ correspond to the inversion grating's amplitudes. An analogous ansatz with $\rho_{i j}^{0}$ and $\rho_{i j}^{ \pm}$is also chosen for off-diagonal density matrix elements which are associated with two closely aligned levels and are thus not treated in RWA, such as resonant tunneling transitions in QCLs!62 Inserting Eqs. 113, 121) and 122 into Eq. 17) with the diffusion term Eq. (120) added to Eq. $17 \mathrm{~b}$, we obtain in a similar way as described in Sec- 
tion IIIE

$$
\begin{aligned}
\partial_{t} \eta_{i j}^{ \pm}= & \mathrm{i} \Delta_{i j} \eta_{i j}^{ \pm}+\frac{\mathrm{i}}{2 \hbar}\left(\rho_{j j}^{0}-\rho_{i i}^{0}\right) \mathbf{d}_{i j}\left\{\begin{array}{l}
\underline{\mathbf{E}}_{+}^{*} \\
\underline{\mathbf{E}}_{ \pm}^{*}
\end{array}\right\} \\
& +\frac{\mathrm{i}}{2 \hbar} \mathbf{d}_{i j}\left\{\begin{array}{l}
\left(\rho_{j j}^{ \pm}-\rho_{i i}^{ \pm}\right) \underline{\mathbf{E}}_{\mp} \\
\left(\rho_{j j}^{\mp}-\rho_{i i}^{\mp}\right) \underline{\mathbf{E}}_{\mp}^{*}
\end{array}\right\}-\gamma_{i j} \eta_{i j}^{ \pm}, \omega_{i j}\left\{\begin{array}{l}
>0 \\
<0
\end{array}\right\}, \\
\partial_{t} \rho_{i i}^{0}= & \frac{1}{\hbar} \sum_{j} \Im\left\{\mathbf{d}_{j i}\left(\eta_{i j}^{+} \underline{\mathbf{E}}_{+}^{*}+\eta_{i j}^{-} \underline{\mathbf{E}}_{-}^{*}\right)\right\} \\
& +\frac{1}{\hbar} \sum_{\omega_{i j}>0} \Im\left\{\mathbf{d}_{j i}\left(\eta_{i j}^{+} \underline{\mathbf{E}}_{+}+\eta_{i j}^{-} \underline{\mathbf{E}}_{-}\right)\right\} \\
& +\sum_{j \neq i} r_{j \rightarrow i} \rho_{j j}^{0}-r_{i} \rho_{i i}^{0}, \\
\partial_{t} \rho_{i i}^{+}= & \frac{\mathrm{i}}{2 \hbar} \sum_{j}\left(\mathbf{d}_{i j} \eta_{j i}^{-} \underline{E}_{+}-\mathbf{d}_{j i} \eta_{i j}^{+} \underline{E}_{-}^{*}\right) \\
& +\frac{\mathrm{i} \omega_{i j}>0}{2 \hbar} \sum_{j}\left(\mathbf{d}_{i j} \eta_{j i}^{+} \underline{E}_{-}^{*}-\mathbf{d}_{j i} \eta_{i j}^{-} \underline{E}_{+}\right) \\
& +\sum_{j \neq i} r_{j \rightarrow i} \rho_{j j}^{+}-r_{i} \rho_{i i}^{+}-4 \beta_{0}^{2} D_{i} \rho_{i i}^{+},
\end{aligned}
$$

with $\Delta_{i j}=\operatorname{sgn}\left(\omega_{i j}\right)\left(\omega_{\mathrm{c}}-\left|\omega_{i j}\right|\right)$. Furthermore, we have $\rho_{i i}^{-}=\left(\rho_{i i}^{+}\right)^{*}, \eta_{j i}^{ \pm}=\left(\eta_{i j}^{ \pm}\right)^{*}$.

Assuming that the coefficients $\beta_{n}$ in Eq. 110 refer to forward propagation, i.e., $\beta_{0}>0$ and $\beta_{1}=v_{\mathrm{g}}^{-1}>0$, the backward propagating field is described by coefficients $-\beta_{n}$. Furthermore deriving the polarization term analogously to Eq. (64), we can summarize the equations for the forward and backward propagating fields as

$$
\begin{aligned}
\frac{1}{v_{\mathrm{g}}} \partial_{t} \underline{E}_{ \pm} \pm \partial_{z} \underline{E}_{ \pm}=\mathrm{i} \sum_{n \geq 2} \frac{\beta_{n}}{n !}\left(\mathrm{i} \partial_{t}\right)^{n} \underline{E}_{ \pm}-\frac{a}{2} \underline{E}_{ \pm} \\
+\mathrm{i} \frac{n_{3 \mathrm{D}} \omega_{\mathrm{c}}^{2}}{\epsilon_{0} \beta_{0} c^{2}} \Gamma \sum_{\omega_{i j}>0} d_{j i} \eta_{i j}^{ \pm},
\end{aligned}
$$

where we have neglected the self-phase modulation term. Equation (124) has to be complemented by the boundary conditions Eq. (114), which together with Eq. (123) constitute the MB model in RWA and SVAA for a waveguide resonator.

This treatment of SHB can be extended by considering higher spatial frequencies of the inversion grating in Eq. 122. ${ }^{208}$ Furthermore, it has been suggested to consider the formation of a grating, and its relaxation due to diffusion, also for the off-diagonal density matrix elements in Eq. 121) 209

\section{ANALYTICAL SOLUTIONS}

\section{A. Rotating Wave Approximation}

\section{Monochromatic Excitation}

The Bloch equations in RWA, Eq. 43), are in principle analytically solvable for monochromatic excitation, corresponding to a time-constant field envelope. 491210 This is usually achieved by using the Laplace transform, which takes a time dependent function $f(t)$ to a function $\mathcal{L}\{f\}(s)$ of a complex frequency variable $s$. The main advantage is that differentiation becomes a multiplication with $s$, i.e., $\mathcal{L}\left\{\partial_{t} f\right\}=s \mathcal{L}\{f\}-f(t=0+)$. Restricting ourselves to a two-level system with initial conditions $w_{0}=w(t=0), \eta_{21}^{0}=\eta_{21}(t=0)$, and considering that the transform is linear and $\mathcal{L}\{1\}=s^{-1}$, Eq. 73 becomes in Laplace domain

$$
\begin{aligned}
\mathcal{L}\left\{\eta_{21}\right\} & =s_{-}^{-1}\left(-\mathrm{i} \mathcal{L}\{w\} \underline{\Omega} / 2+\eta_{21}^{0}\right), \\
\mathcal{L}\left\{\eta_{12}\right\} & =s_{+}^{-1}\left[\mathrm{i} \mathcal{L}\{w\} \underline{\Omega}^{*} / 2+\left(\eta_{21}^{0}\right)^{*}\right] \\
\mathcal{L}\{w\} & =\frac{2 s s_{0}+s_{+} s_{-}\left(\gamma_{1} w_{\mathrm{eq}}+s w_{0}\right)}{s\left[s s_{+} s_{-}+\left(s+\gamma_{2}\right)|\Omega|^{2}+\gamma_{1} s_{+} s_{-}\right]},
\end{aligned}
$$

with $s_{ \pm}=\left(s+\gamma_{2} \pm \mathrm{i} \Delta\right)$ and $s_{0}=\Im\left\{\eta_{21}^{0} \underline{\Omega}^{*}\right\}\left(s+\gamma_{2}\right)+$ $\Delta \Re\left\{\eta_{21}^{0} \Omega^{*}\right\}$. Here, the off-diagonal matrix elements have already been eliminated in Eq. (125c) by inserting Eqs. 125a and 125b. Back-transformation of Eq. $(125 \mathrm{c})$ is achieved by performing a partial fraction decomposition, which results in a sum of simpler fractions with known inverse Laplace transforms. This requires finding the poles of the rational function in Eq. (125c), given by $s=0$ and the roots of the cubic function of $s$ in the square brackets of the denominator, for which closed analytical expressions are readily available. The solution for $f(t)=w(t)$ and $f(t)=\eta_{21}(t)$ is of the form $\frac{49}{4}$

$f(t)=A+B \exp (-a t)+[C \cos (\omega t)+D \sin (\omega t)] \exp (-b t)$,

where the decay constants $a, b$ and the oscillation frequency $\omega$ are the same for $w$ and $\eta_{21}$, but the coefficients $A, B, C$ and $D$ are different and also depend on the initial conditions.

a. Rabi Oscillations In the following, we consider the case of dissipationless light-matter interaction, i.e., $\gamma_{1}=\gamma_{2}=0$. Then, Eq. 125c becomes

$$
\begin{aligned}
\mathcal{L}\{w\} & =\frac{2 s_{0}+\left(s^{2}+\Delta^{2}\right) w_{0}}{s\left(s+\mathrm{i} \Omega_{\mathrm{g}}\right)\left(s-\mathrm{i} \Omega_{\mathrm{g}}\right)} \\
& =\frac{A}{s}+\frac{\left(w_{0}-A\right) s+2 \Im\left\{\eta_{21}^{0} \Omega^{*}\right\}}{s^{2}+\Omega_{\mathrm{g}}^{2}},
\end{aligned}
$$

where $\Omega_{\mathrm{g}}=\left(\Delta^{2}+|\underline{\Omega}|^{2}\right)^{1 / 2}$ denotes the generalized Rabi frequency for detuned excitation, and $A=$ 


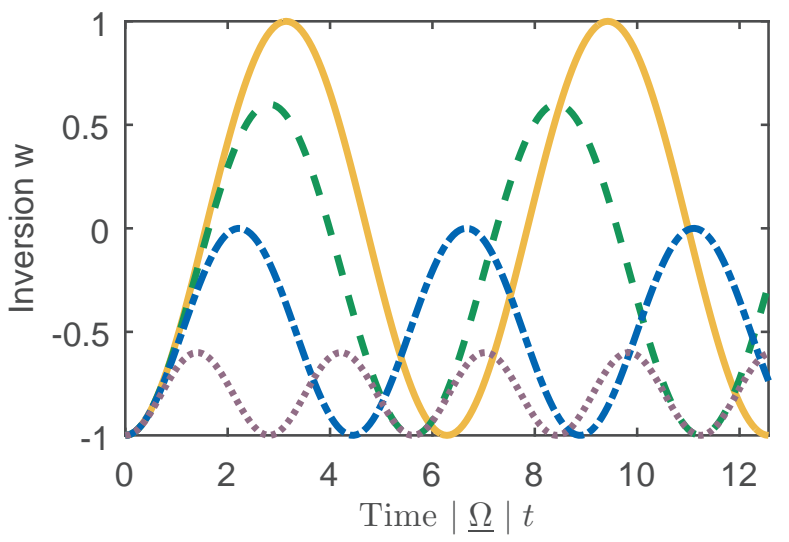

FIG. 12. Time dependent inversion for monochromatic excitation with a field of amplitude $\underline{\Omega}$ and a detuning of $\Delta=0$ (solid line), $\Delta=|\Omega| / 2$ (dashed line), $\Delta=|\underline{\Omega}|$ (dash-dotted line), and $\Delta=2|\underline{\Omega}|$ (dotted line).

$\Omega_{\mathrm{g}}^{-2}\left(2 \Delta \Re\left\{\eta_{21}^{0} \Omega^{*}\right\}+\Delta^{2} w_{0}\right)$. Inverse Laplace transformation of Eq. (126) yields

$w(t)=A+\left(w_{0}-A\right) \cos \left(\Omega_{\mathrm{g}} t\right)+2 \Omega_{\mathrm{g}}^{-1} \Im\left\{\eta_{21}^{0} \underline{\Omega}^{*}\right\} \sin \left(\Omega_{\mathrm{g}} t\right)$.

As can be seen from Eq. 127), a monochromatic, nearresonant light field interacting with an ideal, dissipationless two-level system causes Rabi flopping, i.e., an oscillation of the population between states 1 and 2 with frequency $\Omega_{\mathrm{g}}$, as predicted by I. I. Rabi for the analogous case of a two-level system in a rotating magnetic field. ${ }^{14]}$ In Fig. 12, $w(t)$ is shown for the initial conditions $w_{0}=-1, \eta_{21}^{0}=0$ and different detunings. As can be seen, complete population inversion with $w=1$ is achieved only for resonant excitation.

Due to the presence of dissipation, above presented analytical treatment of Rabi flopping can rarely be directly used for the description of optoelectronic device operation. However, under favorable conditions, signatures of Rabi oscillations have been observed in nanostructured optoelectronic systems and devices. These includes quantum well structures, $\frac{16] 17}{16 C L s}, \frac{18}{18}$ single quantum dots, $\frac{19120}{1}$ and nanowire lasers at cryogenic temperatures, as well as quantum dot ${ }^{2122}$ and quantum dash 23 amplifiers at room temperature. Besides the usually strong influence of dissipation, effects beyond the two-level dynamics and inherent restrictions of models based on macroscopic Maxwell-Bloch equations, the applicability of Eq. 126 is also limited by the validity range of the RWA. In particular, for very strong optical excitation where the Rabi frequency approaches the optical resonance frequency, effects beyond the RWA have been observed in bulk and nanostructured semiconductors 1051211,213

b. Steady State Solution In the following, we consider the steady state behavior for a dissipative two-level system under monochromatic optical excitation, i.e., for a field at frequency $\omega_{\mathrm{c}}$ with constant amplitude. In the presence of dissipation, the coherent transients associated with above discussed Rabi oscillation, Eq. (127), decay, and the system approaches the steady state for $t \rightarrow \infty$. The steady state solution can be obtained by setting $\partial_{t}=0$ in Eq. $733 \cdot{ }^{157}$ Alternatively, we can apply the final value theorem to Eq. 125], stating that if $\lim _{t \rightarrow \infty} f(t)$ exists, it is identical to $\lim _{s \rightarrow 0} s \mathcal{L}\{f\}$. Introducing the relaxation times $T_{1,2}=\gamma_{1,2}^{-1}$, we then obtain

$$
\begin{aligned}
\eta_{21} & =\frac{1}{2} \frac{\underline{\Omega} T_{2}\left(\Delta T_{2}-\mathrm{i}\right) w_{\mathrm{eq}}}{1+\Delta^{2} T_{2}^{2}+T_{1} T_{2}|\underline{\Omega}|^{2}}, \\
w & =\frac{\left(1+\Delta^{2} T_{2}^{2}\right) w_{\mathrm{eq}}}{1+\Delta^{2} T_{2}^{2}+T_{1} T_{2}|\underline{\Omega}|^{2}} .
\end{aligned}
$$

From Eq. 128, an expression for the relative permittivity $\underline{\epsilon}_{\mathrm{r}}$ and susceptibility $\underline{\chi}=\underline{\epsilon}_{\mathrm{r}}-1$ due to the quantum systems can be derived. Setting the classical expression for the complex polarization amplitude $\underline{\mathbf{P}}=\epsilon_{0} \underline{\chi \mathbf{E}}$ equal to Eq. 75 , we obtain with Eq. 128a, $\underline{\Omega}=\hbar^{-1} \mathbf{d}_{21} \underline{\mathbf{E}}$ and Eq. (890) the frequency and intensity dependent susceptibility $157 \mid 170$

$$
\underline{\chi}=\frac{n_{3 \mathrm{D}}\left|d_{21}\right|^{2} T_{2}}{\epsilon_{0} \hbar} \frac{\left(\Delta T_{2}-\mathrm{i}\right) w_{\mathrm{eq}}}{1+\Delta^{2} T_{2}^{2}+I / I_{\mathrm{s}}},
$$

with the saturation intensity at zero detuning

$$
I_{\mathrm{s}}=\frac{\hbar^{2} \epsilon_{0} n_{\mathrm{eff}} c}{2 T_{1} T_{2}\left|d_{21}\right|^{2}} .
$$

For arbitrary detuning, the saturation intensity is then given by $I_{\mathrm{S}}\left(1+\Delta^{2} T_{2}^{2}\right) \stackrel{157}{1}$ i.e., non-resonant fields interact less strongly with the two-level system, and thus saturation occurs for higher intensities. As in Section $\mathrm{VB}$, it is here assumed that $d_{21}$ and $\underline{E}$ are aligned in the same direction, or that $d_{21}$ is an effective value averaged over the different orientations of, e.g., dopant ions in an optical fiber.

In Fig. 13 the real and imaginary parts of $\chi \underline{\chi}$ are shown as a function of detuning from the optical resonance frequency for various optical intensities. $\Re\{\chi\}$, which contains chromatic dispersion, changes sign at the resonance frequency. $\Im\{\underline{\chi}\}$ describes gain for $w_{\text {eq }}>0$, i.e., positive population inversion, and loss for $w_{\text {eq }}<0$, where the frequency dependence is given by a Lorentzian profile with the full width at half-maximum (FWHM) bandwidth $2 \gamma_{2}\left(1+I / I_{\mathrm{S}}\right)^{1 / 2}$. For increased intensities, the profile thus gets broadened which is known as power broadening, and also the peak value at resonance frequency is reduced by a factor of $1+I / I_{\mathrm{s}}$, which corresponds to gain saturation for $w_{\text {eq }}>0$ and saturable absorption for $w_{\text {eq }}<0$.

In the following, we investigate optical power amplification or absorption by two-level systems. For TE modes or generally in the limit of weak waveguiding, the power is given by Eq. (91). Multiplying Eq. (110) from left with $E^{*}$ and adding the complex conjugate, we obtain with Eqs. 128a) and (91) and $\beta_{0}=\omega_{\mathrm{c}} n_{\mathrm{eff}} / c$

$$
\partial_{z} P=-a P+g P,
$$



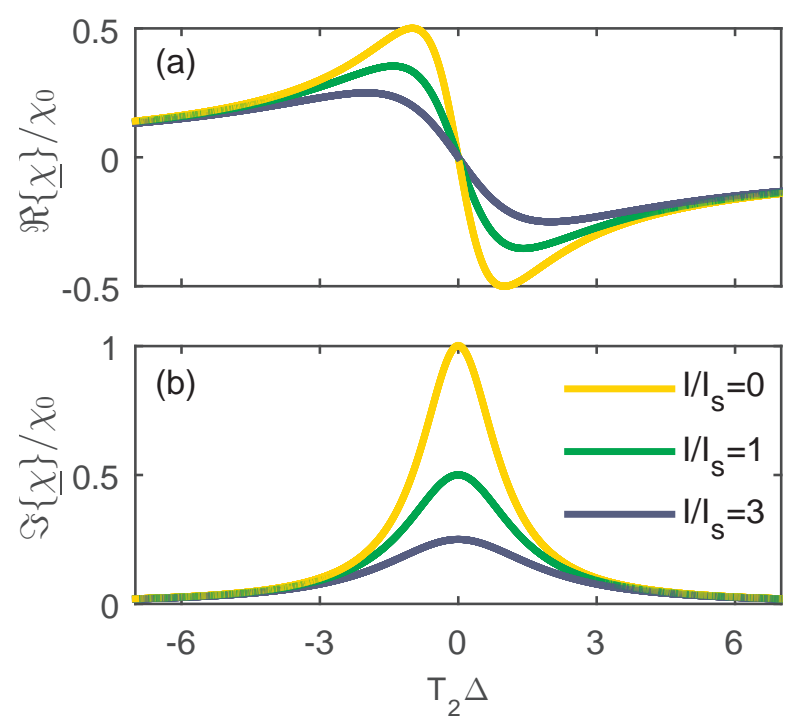

FIG. 13. Complex susceptibility of the two-level system, normalized to $\chi_{0}=-n_{3 \mathrm{D}}\left|d_{21}\right|^{2} T_{2} \epsilon_{0}^{-1} \hbar^{-1} w_{\text {eq }}$, as a function of the detuning frequency $\Delta$. Shown is the (a) real and (b) imaginary part for various optical intensities.

where $a$ is the waveguide loss coefficient, and the twolevel power gain coefficient is given by

$$
g=\alpha \frac{w_{\mathrm{eq}}}{1+\Delta^{2} T_{2}^{2}+P / P_{\mathrm{s}}},
$$

with

$$
\alpha=\Gamma \frac{\omega_{\mathrm{c}} n_{3 \mathrm{D}}\left|d_{21}\right|^{2} T_{2}}{\epsilon_{0} c \hbar n_{\mathrm{eff}}}
$$

and the saturation power at zero detuning $P_{\mathrm{s}}=A_{\text {eff }} I_{\mathrm{s}}$. With the help of the Lambert $\mathrm{W}$ function, defined by $x=$ $\mathrm{W}(x) \exp [\mathrm{W}(x)]$, we can write the solution of Eqs. (131) and 132 for zero waveguide loss, $a=0$, as $P(z)=$ $P_{0} G(z)$ with the power gain factor

$$
G(z)=\frac{P_{\mathrm{s}}^{\prime}}{P_{0}} \mathrm{~W}\left[\frac{P_{0}}{P_{\mathrm{s}}^{\prime}} \exp \left(\frac{P_{0}}{P_{\mathrm{s}}^{\prime}}\right) \exp \left(\frac{\alpha w_{\mathrm{eq}}}{1+\Delta^{2} T_{2}^{2}} z\right)\right],
$$

where $P_{0}=P(z=0)$ and $P_{\mathrm{s}}^{\prime}=\left(1+\Delta^{2} T_{2}^{2}\right) P_{\mathrm{s}}$. With Eq. (134), the steady state field solution of Eq. 110) can then for $a=\gamma=0$ be written as

$$
\underline{E}(z)=\underline{E}(z=0)[G(z)]^{\left(1+\mathrm{i} \Delta T_{2}\right) / 2},
$$

and the density matrix elements are with $\underline{\Omega}=\hbar^{-1} d_{21} \underline{E}$ given by Eq. (128). In the exponent of Eq. (135), $\alpha_{\mathrm{H}}=$ $-\Delta T_{2}$ corresponds to the Henry or linewidth enhancement factor, 214 which relates phase changes to changes in the optical gain.

In Fig.14 the optical power gain and phase shift of the electric field are shown as a function of propagation distance for different values of initial power $P_{0}$ for $w_{\text {eq }}>0$, i.e., amplification. In the small signal limit,
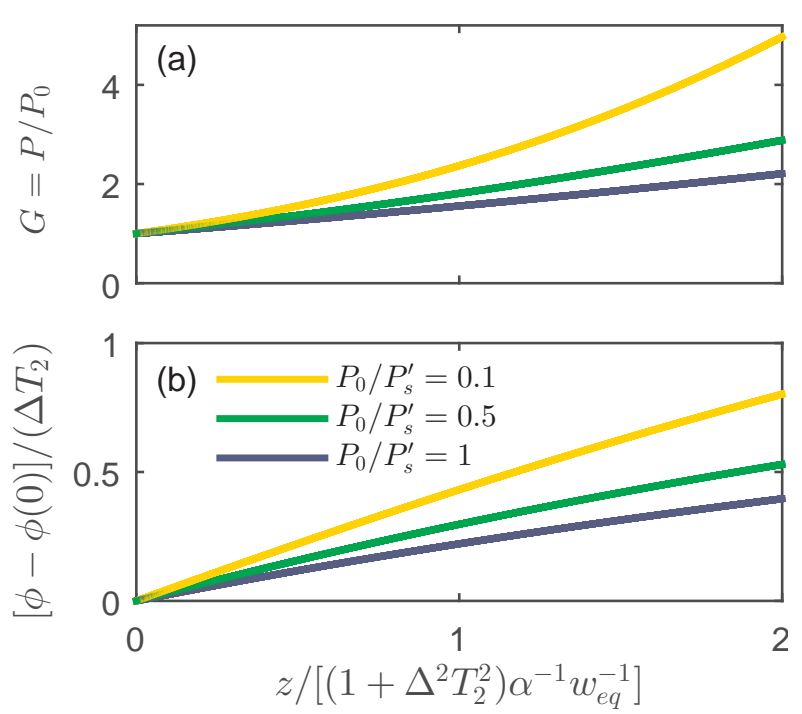

FIG. 14. (a) Optical power gain and (b) phase shift of the electric field as a function of propagation distance for different values of initial power.

$P \ll P_{\mathrm{s}}^{\prime}$, the typical exponential increase in power is observed. This can also be seen from Eq. 134, which yields with $\mathrm{W}(x) \approx x$ for $x \ll 1$ the usual exponential amplification (for $G>1$ ) or loss (for $G<1$ ) characteristics. In the saturation regime, the power increases only linearly. Physically, this is a consequence of the fact that the growth in optical power is ultimately limited by the supplied pump power.

The expressions Eqs. 129 and 132 for the susceptibility $\chi$ and optical gain $g$ are widely used to model the optical properties of homogeneously broadened atomic $\sqrt{157 / 170}$ and nanostructured ${ }^{215 / 216}$ optical media. In particular for interband transitions in bulk semiconductor and quantum well media, the electron wavevector must be explicitly considered, along with additional corrections due to Coulomb interactions ${ }^{6567}$ Above derivation of an expression for the susceptibility from the Bloch equations can be extended to more than two levels, which is for example relevant for the investigation of slow light propagation. This is usually achieved based on electromagnetically induced transparency (EIT), where a control laser beam induces a narrow transparency window with an extremely low group velocity in the absorption spectrum of a suitable medium.217.219 EIT requires a three-level configuration, and expressions for the susceptibility have been derived in a similar way as above.$^{35 \mid 219}$ A reduction of the group velocity to subsonic speeds, as well as complete halting of light, has been demonstrated in an ultra-cold atomic vapor ${ }^{32} 34$ Possible applications include optical buffers, ${ }^{35}$ imaging, ${ }^{36 / 37}$ and quantum memory ${ }^{38}$ In view of a future commercialization of these technologies, a compact solid-state based implementation is desirable, and slow light propagation as well as light trapping has meanwhile been demonstrated in doped crystals $\frac{39140}{}$ The realization of slow 
light in suitably engineered semiconductor structures is especially attractive. Here, the exploitation of tunneling induced transparency is highly promising, which differs from EIT in that it does not require an optical control field, but utilizes strong tunneling coupling between a pair of states. For this case, the susceptibility has been analytically derived for quantum dot and intersubband quantum well systems. $\stackrel{41}{43}$ Furthermore, nonlinear optical mixing effects which involve optical field contributions at two or more frequencies, and often rely on more than two energy levels, are exploited in many semiconductorbased applications, requiring a description by higher order susceptibilities ${ }^{220}{ }^{227}$ The corresponding expressions can for example be obtained from the Bloch equations by employing time dependent perturbation theory $228 \mid 229$

\section{Self-Induced Transparency}

In addition to above presented steady state solution to the Maxwell-Bloch equations, also dynamic solutions are available for some special cases. An important example is self-induced transparency (SIT), where a special optical pulse solution exists which can propagate through the two-level medium without being attenuated or disturbed. This effect was theoretically predicted, and first experimentally demonstrated in ruby, by McCall and Hahn.2425 SIT is based on coherent interaction with the medium, which requires that the pulse duration must be much shorter than the relaxation processes described by $\gamma_{1}$ and $\gamma_{2}$, and thus we can set $\gamma_{1}=\gamma_{2}=0$. Furthermore we assume that the field envelope $\underline{\Omega}=\hbar^{-1} d_{21} \underline{E}$ is real-valued, and initially restrict ourselves to resonant excitation, i.e., $\Delta=0$. Then, the solution of Eq. (74) for the initial condition $s_{1}(z,-\infty)=s_{2}(z,-\infty)=0, w(z,-\infty)=-1$ can be written as $s_{1}=0, s_{2}=-\sin \vartheta$ and $w=-\cos \vartheta$ with $\vartheta(z, t)=\int_{-\infty}^{t} \underline{\Omega}\left(z, t^{\prime}\right) \mathrm{d} t^{\prime}$, as can easily be verified by re-insertion of the solution into Eq. (74). This analysis can be extended to incorporate inhomogeneous broadening in media consisting of quantum systems with slightly different resonance frequencies $49 \mid 109$ Assuming that non-resonant systems with $\Delta \neq 0$ essentially respond in the same way to $\underline{\Omega}$ as the resonant ones, apart from a change in amplitude, we can make the factorization ansatz $s_{2, \Delta}(z, t)=F(\Delta) s_{2}(z, t)$, which again yields closed analytical solutions to Eq. (74),

$$
\begin{aligned}
s_{2, \Delta}(z, t) & =-F(\Delta) \sin \vartheta(z, t), \\
w_{\Delta}(z, t) & =-F(\Delta)[\cos \vartheta(z, t)-1]-1 .
\end{aligned}
$$

Taking the second derivative of Eq. $74 \mathrm{~b}$ for $\Delta \neq 0$ and inserting Eqs. 174a), 136 and 137 yields

$$
\partial_{t}^{2} \vartheta=\frac{\Delta^{2} F(\Delta)}{1-F(\Delta)} \sin \vartheta:=T^{-2} \sin \vartheta .
$$

Since the electric field envelope, and hence also $\vartheta$, is $\Delta$ independent, this must also apply to $\Delta^{2} F(\Delta) /[1-F(\Delta)]$

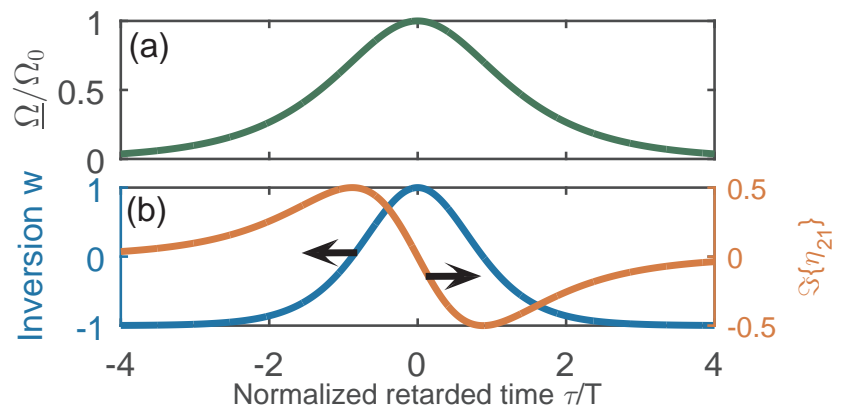

FIG. 15. Self-induced transparency soliton. Shown are (a) the normalized electromagnetic field and (b) inversion $w$ and imaginary part of the off-diagonal matrix element $\eta_{21}$, where $\Re\left\{\eta_{21}\right\}=0$.

which we have thus set equal to a constant $T^{-2}$ in Eq. 138). This yields a Lorentzian dependence $F=$ $1 /\left(1+T^{2} \Delta^{2}\right)$. Equation 138 corresponds to the pendulum problem, where the solutions are given by elliptic functions. Here we require $\underline{\Omega}=\partial_{t} \underline{\Omega}=0$ and thus $\partial_{t} \vartheta=\partial_{t}^{2} \vartheta=0$ at $t= \pm \infty$, yielding the unique solution $\vartheta=4 \arctan \left\{\exp \left[\left(t-t_{0}\right) / T\right]\right\}$. Introducing $t_{0}=z / v$ with the pulse propagation velocity $v$, we thus obtain

$$
\underline{\Omega}(z, t)=2 T^{-1} \operatorname{sech}(\tau / T)
$$

with the retarded time variable $\tau=t-z / v$.

In the optical propagation equation, inhomogeneous broadening can approximately be included by substituting $s_{1}-\mathrm{i} s_{2}$ with $\int_{-\infty}^{\infty} g(\Delta)\left(s_{1, \Delta}-\mathrm{i} s_{2, \Delta}\right) \mathrm{d} \Delta$ in the polarization, Eq. 75 , where $g(\Delta)$ with $\int_{-\infty}^{\infty} g(\Delta) \mathrm{d} \Delta=1$ gives the distribution of quantum systems as a function of the detuning $\Delta$ from $\omega_{\mathrm{c}}$. Here, a possible dependence of the dipole matrix element on $\Delta$ has been neglected. Using $s_{2, \Delta}=F(\Delta) s_{2}$ and above result for $F(\Delta)$, we obtain $s_{2,-\Delta}=s_{2, \Delta}$, and with Eq. 74a we see that then $s_{1,-\Delta}=-s_{1, \Delta}$ for $\underline{\Omega}_{\mathrm{i}}=\gamma_{2}=0$. Often $g(\Delta)$ is an even function as further discussed in Section VIIIB and then the contribution of $s_{1, \Delta}$ cancels out. Under this assumption, Eq. 110 becomes without dispersion $\left(\beta_{n}=0\right.$ for $n \geq 2)$, $\operatorname{loss}(a=0)$ and self-phase modulation $(\gamma=0)$

$$
\partial_{t} \underline{\Omega}+v_{\mathrm{g}} \partial_{z} \underline{\Omega}=2^{-1} \alpha \gamma_{2} v_{\mathrm{g}} s_{2} \int_{-\infty}^{\infty} g(\Delta) F(\Delta) \mathrm{d} \Delta,
$$

where $\alpha$ is given by Eq. (133). Inserting Eqs. 1139$)$ and (136) into Eq. 140 yields the pulse propagation velocity

$$
v=v_{\mathrm{g}} \frac{2}{2+\alpha \gamma_{2} v_{\mathrm{g}} T^{2} \int_{-\infty}^{\infty} g(\Delta)\left(1+T^{2} \Delta^{2}\right)^{-1} \mathrm{~d} \Delta} .
$$

In Fig.15 the pulse shape, inversion $w$ and the imaginary part of the off-diagonal matrix element $\Im\left\{\eta_{21}\right\}=$ $-s_{2} / 2$ is shown. Notably, for $w_{\text {eq }}<0$ when the two-level medium normally absorbs light [see Eq. [132] ], the optical energy absorbed during the first half of the SIT pulse 
and stored in the inversion, is re-emitted during the second half, which delays the pulse so that $v$ is smaller than the group velocity $v_{\mathrm{g}}$ without the coherent interaction as can be seen from Eq. 141, but does not change its shape or amplitude. This is accompanied by a Rabi flop of the population inversion from $w=-1$ to $w=1$ and back again.

Generally, based on the area theorem it was found that for coherent propagation, the pulse area $\Theta=\int \underline{\Omega} \mathrm{d} t$ evolves towards the closest even multiple of $\pi(\Theta=$ $0,2 \pi, 4 \pi, \ldots)$ for absorbing media $\left(w_{\text {eq }}<0\right)$, and to the closest odd multiple of $\pi$ for gain media $\left(w_{\text {eq }}>0\right) ! 24$ Importantly, the area theorem only makes a statement about $\Theta$, but does not indicate if the pulse envelope changes. As discussed above, the SIT pulse Eq. (139), which has a pulse area $\Theta=2 \pi$, is the only finite energy solution of Eq. 138 where the pulse envelope is preserved. However, analytical solutions of the MB equations with changing pulse shapes can also be obtained for other cases of coherent propagation.230|231

With regards to novel practical applications, SIT is for example a highly interesting candidate for the generation of ultrashort optical pulses in various types of lasers with sufficiently long coherence times, such as quantum dot and quantum cascade lasers.27|[31|121|232|233 This SIT (or coherent) mode-locking approach requires a laser design with one or multiple gain and absorber regions, where an SIT soliton with a pulse area of $d_{21} \int \underline{E} \mathrm{~d} t / \hbar=2 \pi$ is approximately realized in the absorber sections. In order to obtain a stable pulse area of $\pi$ in the gain regions, they are engineered to have half the dipole moment $d_{21}$ of the absorber sections. Instead of sequential gain and absorber regions, another option is to stack the gain and loss regions in transverse direction, i.e., perpendicular to the propagation axis. This approach is for example compatible with the manufacturing process of QCLs, and an analytical solution has been derived for the steady state pulse solution ${ }^{30}$ Despite its great promise, SIT mode-locking has not been experimentally demonstrated to date.

\section{B. Full-Wave Bloch Equations}

Without employing the RWA, the Bloch equations (17) are solvable only for very special conditions. In particular, for $|\Delta M|=1$ transitions in hydrogen-like atoms where the dipole matrix element is given by $\mathbf{d}=$ $2^{-1 / 2}|\mathbf{d}|\left(\mathbf{e}_{x}-\mathbf{i} \mathbf{e}_{y}\right)$, excitation with circularly polarized light where $E_{x}=E(t) \cos (\omega t)$ and $E_{y}=E(t) \sin (\omega t)$ leads to $\mathbf{d E}(t)=2^{-1 / 2}|\mathbf{d}| E(t) \exp (-\mathrm{i} \omega t){ }^{14}|49|$ Furthermore using Eq. 42 to substitute the off-diagonal density matrix elements in Eq. (17), the resulting equation formally corresponds to the RWA Bloch equation, with the analytical solutions discussed in Section VIA. Closed analytical solutions are not available for the relatively simple, but very important case of monochromatic excitation with a linearly polarized wave. Some approximate corrections have been derived, such as the Bloch-
Siegert shift describing the change in the system's resonance frequency for strong driving, 155 and the Mollow triplet which refers to the emergence of satellite peaks in the spectrum of resonantly excited systems.234 Interestingly, the full Bloch equations can be solved analytically if the linearly polarized electric field has the form of an $N$-soliton. This is also true for the so-called reduced MB equations, which combine the full-wave Bloch equations with a first-order unidirectional optical propagation equation. $235 \mid 236$

\section{NUMERICAL SCHEMES}

As discussed in Section VI, the full-wave MB equations have known analytical solutions only for very special cases, and also in the RWA/SVAA approximation, no general analytical solution exists. Therefore computer simulations are in general necessary. From a practical point of view, the numerical scheme should be stable, accurate, and efficient, and a naive discretization will often fail. The goal of this section is to introduce wellestablished approaches which are straightforward to implement, and give a critical discussion of their properties. Furthermore, an overview of recent developments in the field will be given. Since the RWA/SVAA problem and the full MB equations are not of the same mathematical form, their numerical implementation has to be treated separately.

Several software projects have been published that are able to solve the Maxwell-Bloch equations. For example, the Freetwm too ${ }^{237}$ is an open-source MATLAB code that simulates the dynamics of semiconductor lasers using the $1 \mathrm{D} \mathrm{MB}$ equations in rotating wave approximation. The Electromagnetic Template Library (EMTL) is a free $\mathrm{C}++$ library with Message Passing Interface (MPI) support,$\stackrel{238}{ }$ which has for example been used to model quantum emitters with the full-wave MB equations in two dimensions. 239 Another solver library for the full-wave MB equations is the open-source MEEP project, 240 using a similar representation of the Bloch equations as given in Eq. 72. The mbsolve project ${ }^{241}$ solves the full-wave MB equations using different parallel acceleration techniques and features an open-source codebase. Finally, a commercial MB solver has been announced.242

\section{A. Rotating Wave/Slowly Varying Amplitude Approximation}

\section{Finite Difference Discretization of the One-Dimensional Propagation Equation}

In the following, the numerical solution of the onedimensional optical propagation equation in the SVAA is discussed. Neglecting chromatic dispersion, i.e., setting $\beta_{n}=0$ for $n \geq 2$, we write Eq. 124 in the form

$$
\partial_{t} E^{ \pm}=\mp v_{\mathrm{g}} \partial_{z} E^{ \pm}+f^{ \pm}(z, t)-\ell E^{ \pm} .
$$


An obvious choice is to use a finite difference discretization approach where a full spatiotemporal discretization of $E^{ \pm}$onto an equidistant grid with $z_{m}=m \Delta_{z}, t_{n}=n \Delta_{t}$ is imposed. In the following, $E_{m, n}^{ \pm}$and $f_{m, n}^{ \pm}$denote the numerical solution of $E^{ \pm}$and $f^{ \pm}$on the grid. The starting point is a Taylor series expansion of $E^{ \pm}\left(z_{m}, t_{n+1}\right)$ around the point $\left(z_{m}, t_{n}\right)$, yielding up to second order

$$
E_{m, n+1}^{ \pm}=E_{m, n}^{ \pm}+\Delta_{t} \partial_{t} E_{m, n}^{ \pm}+\frac{\Delta_{t}^{2}}{2} \partial_{t}^{2} E_{m, n}^{ \pm}
$$

Then $\partial_{t} E_{m, n}^{ \pm}$and $\partial_{t}^{2} E_{m, n}^{ \pm}$are replaced by space derivatives: Differentiating Eq. 142 with respect to $z$, multiplying the result by $\mp v_{\mathrm{g}}$ and adding it to the time derivative of Eq. 142 yields $\left(\partial_{t}^{2}-v_{\mathrm{g}}^{2} \partial_{z}^{2}\right) E^{ \pm}=\left(\partial_{t} \mp v_{\mathrm{g}} \partial_{z}\right)\left(f^{ \pm}-\right.$ $\left.\ell E^{ \pm}\right)$. Plugging the result into Eq. 143 and furthermore using Eq. 142, we obtain

$$
\begin{aligned}
E_{m, n+1}^{ \pm}= & E_{m, n}^{ \pm}+\Delta_{t}\left\{\mp v_{\mathrm{g}}\left[\partial_{z} E^{ \pm}\right]_{m, n}+f_{m, n}^{ \pm}-\ell E_{m, n}^{ \pm}\right\} \\
& +\frac{\Delta_{t}^{2}}{2}\left\{v_{\mathrm{g}}^{2}\left[\partial_{z}^{2} E^{ \pm}\right]_{m, n}+\left[\partial_{t} f^{ \pm}\right]_{m, n}\right. \\
& \left.\mp v_{\mathrm{g}}\left[\partial_{z} f^{ \pm}\right]_{m, n}\right\} \\
& +\frac{\ell \Delta_{t}^{2}}{2}\left\{ \pm 2 v_{\mathrm{g}}\left[\partial_{z} E^{ \pm}\right]_{m, n}-f_{m, n}^{ \pm}+\ell E_{m, n}^{ \pm}\right\} .
\end{aligned}
$$

For finite difference discretization, there are different possibilities such as the well known and widely used $2^{\text {nd }}$ order Lax-Wendroff method, 243 or the Risken-Nummedal finite differences (RNFD) scheme which was specifically developed in the context of MB simulations. 244 In both cases, $\left[\partial_{z}^{2} E^{ \pm}\right]_{m, n}$ is approximated by the standard finite difference approximation $\left(E_{m+1, n}^{ \pm}-2 E_{m, n}^{ \pm}+\right.$ $\left.E_{m-1, n}^{ \pm}\right) / \Delta_{z}^{2}$. Here we will treat in detail the RNFD scheme, since it has some advantageous properties as discussed further below. The main difference as compared to the Lax-Wendroff method is that rather than employing centered differences, depending on the propagation direction backward/forward finite differences are used, with $\left[\partial_{z} E^{ \pm}\right]_{m, n} \approx \pm\left(E_{m, n}^{ \pm}-E_{m \mp 1, n}^{ \pm}\right) / \Delta_{z},\left[\partial_{z} f^{ \pm}\right]_{m, n} \approx$ $\pm\left(f_{m, n}^{ \pm}-f_{m \mp 1, n}^{ \pm}\right) / \Delta_{z}$. Furthermore, a time step of $\Delta_{t}=\Delta_{z} / v_{\mathrm{g}}$ is imposed. From Eq. 144, we then obtain the RNFD scheme

$$
\begin{aligned}
E_{m, n+1}^{ \pm} & =E_{m \mp 1, n}^{ \pm}+\Delta_{t}\left(\frac{1}{2} f_{m, n}^{ \pm}+\frac{1}{2} f_{m \mp 1, n}^{ \pm}-\ell E_{m \mp 1, n}^{ \pm}\right) \\
& +\frac{\Delta_{t}^{2}}{2}\left\{\left[\partial_{t} f^{ \pm}\right]_{m, n}-\ell f_{m, n}^{ \pm}+\ell^{2} E_{m, n}^{ \pm}\right\} .
\end{aligned}
$$

The term $\left[\partial_{t} f^{ \pm}\right]_{m, n}$ is not substituted with a corresponding finite difference approximation since $\partial_{t} \eta_{i j}$ can directly be obtained from the Bloch equations, Eq. 43. In a Fabry-Pérot type resonator, Eq. (145) is complemented by the boundary conditions Eq. 114).

a. Numerical Properties of the RNFD Scheme For a numerical scheme to be useful, an important requirement is that round-off and truncation errors do not get amplified during the computation, since this will eventually lead to numerical instability. The stability of finite difference discretization schemes can be investigated based on a von Neumann stability analysis. ${ }^{245}$ It turns out that the RNFD scheme is stable for $\ell \geq 0$, which is also true for the Lax-Wendroff method for a sufficiently small Courant number $v_{\mathrm{g}} \Delta_{t} / \Delta_{z}$. On the other hand, for positive linear gain, i.e., $\ell<0$, we obtain unconditionally unstable behavior for both schemes. Furthermore, like the Lax-Wendroff method, the RNFD scheme is second order accurate in space and time ${ }^{244}$ This guarantees that the numerical scheme converges to the original partial differential equation as the grid spacing approaches zero, with a convergence order of two. However, this does not yet guarantee that the numerical solution for finite grid spacing has a physically meaningful behavior, e.g., satisfies certain physical conservation laws. Thus, additional conditions might be desirable for a finite difference discretization of Eq. 142, which has the form of an inhomogeneous scalar convection equation and thus allows us to draw from related work. ${ }^{246}$ Specifically, it has been established that second and higher order linear finite difference schemes tend to introduce artificial numerical dispersion, yielding phase errors and numerical oscillations near extrema or discontinuities of the solution. ${ }^{247}$ The numerical solution is less prone to phase errors for monotonicity preserving schemes, which guarantee that for every nondecreasing (non-increasing) initial condition $E_{m, n=0}^{ \pm}$, the numerical solution at all later instants $n>0$ is also nondecreasing (non-increasing). A sufficient condition for the RNFD scheme to be monotonicity preserving for the homogeneous propagation equation, i.e., Eq. 142 with a vanishing source term $f^{ \pm}(z, t) \equiv 0$, can be easily derived: Formulating Eq. 145 for $E_{m+1, n+1}^{ \pm}$and subtracting the resulting expression from Eq. (145), we arrive at

$$
\begin{aligned}
E_{m+1, n+1}^{ \pm}-E_{m, n+1}^{ \pm} & =\left(1-\ell \Delta_{t}\right)\left(E_{m+1 \mp 1, n}^{ \pm}-E_{m \mp 1, n}^{ \pm}\right) \\
& +\frac{\Delta_{t}^{2}}{2} \ell^{2}\left(E_{m+1, n}^{ \pm}-E_{m, n}^{ \pm}\right)
\end{aligned}
$$

which yields $\Delta_{t} \leq 1 / \ell$ as sufficient condition for monotonicity preservation in the stability regime $\ell \geq 0$. This is a unique feature for a second order finite difference propagation scheme which is directly related to the choice of time step $\Delta_{t}=\Delta_{z} / v_{\mathrm{g}}$. Also, this constitutes an important advantage of the RNFD scheme over the LaxWendroff method, which does not have this property in numerically stable regions, as can be shown in a similar way as above or directly from Godunov's order barrier theorem. 247

In Fig.16, the Lax-Wendroff and the RNFD scheme are compared for lossless propagation of an initially rectangular pulse without interaction with a quantum system. For the Lax-Wendroff scheme, spurious oscillatory features arise in the vicinity of the field discontinuities, which are absent in the RNFD scheme due to its monotonicity preserving nature. 


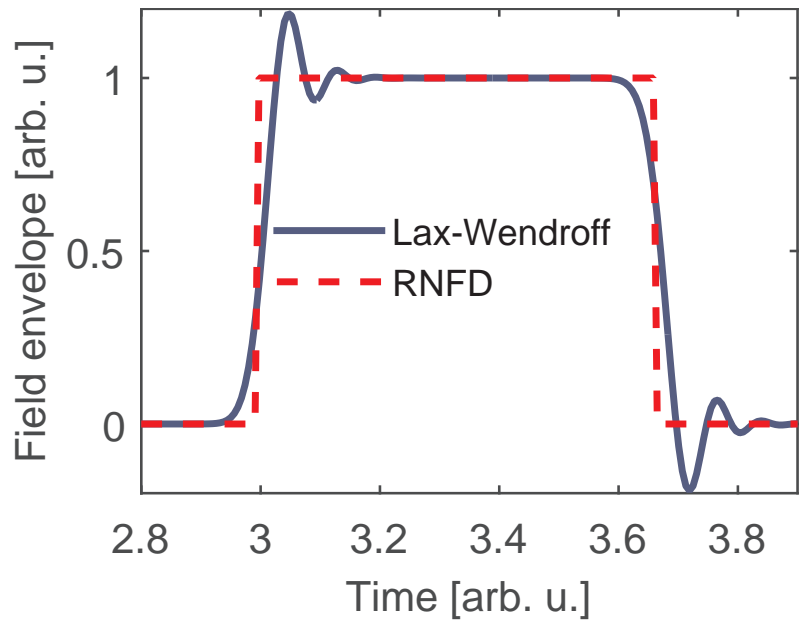

FIG. 16. Comparison between Lax-Wendroff and RNFD schemes for the forward propagation of a rectangular pulse with $f^{+}=\ell=0$.

\section{Density Matrix Equations}

The numerical scheme for the optical propagation equation has to be coupled to a time-propagation scheme for the Bloch equations, Eq. 43. . These constitute an ordinary differential equation (ODE) system describing the temporal evolution of the density matrix, which has to be solved for each spatial grid point. In principle, most standard methods should do the job although they will differ in numerical stability, accuracy and efficiency, and well-established schemes such as Runge-Kutta 248 250 and Adams-Bashforth ${ }^{62}$ have successfully been used. Most research on the suitability of different numerical schemes in literature has focused on the full-wave Bloch equations, as detailed in Section VIIB. Since they are identical in structure to the RWA Bloch equations [compare for example Eqs. (67a) and (68) to Eq. (73)], the obtained insights should in principle also be valid for the RWA Bloch equations. The Runge-Kutta method is further described in Section VIIB 4 in the context of full-wave Bloch equations. Here we exemplarily discuss the explicit Adams-Bashforth scheme as an especially straightforward to implement and numerically highly efficient method. The RNFD scheme for the MB equations, Eq. 145, is strongly coupled, i.e., requires an evaluation of the density matrix and the electric field at the same time value. The $k$-step Adams-Bashforth method for the solution of an ODE system is given by $251+253$

$$
\hat{\rho}^{n+1}=\hat{\rho}^{n}+\Delta_{t} \sum_{m=0}^{k-1} c_{m} \mathcal{F}^{n-m}\left(\hat{\rho}^{n-m}\right) .
$$

Here, $n$ corresponds to the time $t_{n}=n \Delta_{t}, \Delta_{t}$ is the time step size, and $\mathcal{F}(\hat{\rho})=\mathcal{L}(\hat{\rho})+\mathcal{D}(\hat{\rho})$ represents the right hand side of the Lindblad equation (3), and specifically in our case of the RWA Bloch equations, Eq. (43). Furthermore, the $c_{m}$ are suitably chosen coefficients 251 so that maximal accuracy is reached in the approximation. A $k$-step Adams-Bashforth method has a global numerical error on the order of $O\left(\Delta_{t}^{k}\right)\left[\frac{252}{2}\right.$ In this context, it must be considered that the overall numerical accuracy cannot be arbitrarily improved by choosing a high value of $k$, since it is also limited by the numerical discretization of the optical propagation equation, e.g., based on the RNFD method. As discussed in Section IVC the Bloch equations are initialized by the starting values of the density matrix elements at a given time, while the Adams-Bashforth method would require $k$ initial values as can be seen from Eq. 146). This problem can for example be solved by doing the first $k-1$ time steps with a different numerical scheme such as the Runge-Kutta method, or by initializing the simulations with two-step Adams-Bashforth on a finer grid. In simulations of laser operation which are typically started from noise, $\frac{173}{17}$ the exact choice of initial conditions is not critical and thus the initialization steps required by Adams-Bashforth do not pose a problem. The main advantage is the reduced numerical load as compared to the Runge-Kutta method (see Section VII B 4, which however requires initialization only at a single time point.

\section{Generalizations and Alternative Methods}

In Section VII A 1, one-dimensional propagation has been assumed, neglecting the transverse coordinates in the SVAA propagation equation, Eq. 62. In reality, the field dependence, and thus also the temporal evolution of the quantum systems, is varying along the $x$ and $y$ coordinates, which must be explicitly considered for an inclusion of diffraction and other effects! 174|249|254 As long as no transverse boundary conditions or material dependencies have to be considered, i.e., $\Delta_{n}$ and $\sigma$ in Eq. 62 are constant, the most straightforward approach is to Fourier-transform Eq. (62) with respect to $x$ and $y$ before the time propagation step is carried out!249 The resulting equation then depends on $z, t$ and the spatial Fourier frequencies $k_{x}$ and $k_{y}$, converting the derivative operator $\nabla_{\mathrm{T}}^{2}$ into a multiplication with $-\left(k_{x}^{2}+k_{y}^{2}\right)$. Thus a one-dimensional propagation method can be used, such as the one discussed in Section VIIA 1. Since this procedure requires a Fourier transform before and an inverse transform after each propagation step, the numerically efficient fast Fourier transform method is usually employed.

As discussed in the context of Eq. 112, for the modeling of unidirectional fiber or beam propagation often the initial field at $z=0$ is given, and the solution at a certain distance $z=L$ is required. Then it is more practical to propagate the field in $z$ direction rather than in time, and to introduce the retarded time variable $\tau=t-z / v_{\mathrm{g}}$ which simplifies the propagation operator $\left(v_{\mathrm{g}}^{-1} \partial_{t} \underline{E}+\partial_{z} \underline{E}\right)$ to $\partial_{z} \underline{E}$. In the absence of other time derivatives, e.g., due to chromatic dispersion, this effectively reduces the propagation equation to an ODE. The solution is then marched 
in $z$ direction in dependence of $\tau$ (and $k_{x, y}$ if applicable), and the density matrix is updated after every propagation step. 2491250 The propagation along $z$ can be performed with a conventional ODE scheme where for example the Adams-Moulton method (with the trapezoidal rule as a widely used special case) or Adams-Bashforth method, Eq. 146], have been employed, in both cases combined with fourth-order Runge-Kutta for the Bloch equations. ${ }^{249 \mid 250}$ In the more general case where time derivatives have to be considered in Eq. 1112, for example to incorporate chromatic dispersion, these can be handled in Fourier domain, similarly as for the $x$ and $y$ derivatives discussed in the previous paragraph. One option is to process all terms in Fourier domain, 255 which however complicates the treatment of expressions which are nonlinear in the field, such as the self-phase modulation term in Eq. 112 . Another strategy might be to couple the Bloch equations to the split-step Fourier method, which treats only the terms containing time derivatives in Fourier domain, and the others in time domain!181

\section{B. Full-Wave Simulation}

While the RWA significantly reduces the computational workload, care must be taken in cases where its basic assumptions are not fulfilled. For example, the RWA assumes that the electric field intensity is small and the field spectrum narrow. However, in a scenario where ultrashort pulse generation is simulated (e.g., mode-locking operation in quantum cascade lasers), the electric field features high peak intensity and a broad spectrum. In such cases, the full electromagnetic wave might have to be considered in the simulation, and a suitable numerical scheme has to be used. In the following, we describe the methods for the Maxwell and full-wave Bloch equations, Eqs. (44) and (17), which are most widely used in related literature, and address the coupling between the updates of the electric field and the density matrix. Finally, we assess the advantages and drawbacks of the different methods.

\section{Numerical Schemes for Maxwell's Equations}

Out of the many numerical methods that solve Maxwell's Equations, mainly two - namely the finitedifference time-domain (FDTD) and the pseudo-spectral time-domain (PSTD) method - are used in the context of Maxwell-Bloch equations.

The FDTD method is one of the standard methods for Maxwell's equations, $\frac{52}{5}$ and is widely used in combination

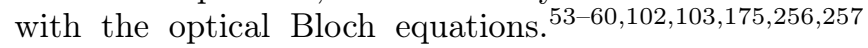
Here, the derivatives with respect to time and space are approximated using central differences. Hence, the method has second order accuracy. In order to facilitate the calculation of the central differences, the Yee grid is used where the discretization points are staggered by half of the respective step size ${ }^{258}$ Figure 17 depicts an example of a Yee grid in one spatial dimension. The main advantage of the FDTD scheme is its simplicity. The implementation of the method as well as boundary conditions or sources is straightforward ${ }^{[52}$ Additionally, it can be executed efficiently in parallel, although the naive implementation will not yield the maximum performance and a more advanced approach must be used 2591260 The major drawback is the introduced numerical dispersion which can only be avoided by using very fine discretization sizes. Otherwise, artifacts in the simulation results could be the consequence. In the context of MB simulations, different values (and value ranges) for the maximal spatial discretization size $\Delta_{z}$ have been found adequate for the FDTD scheme. Namely, $\lambda / 20$ to $\lambda / 100,257$ $\lambda / 50 \stackrel{151}{151} \lambda / 100, \stackrel{261}{ }$ and $\lambda / 20{ }^{[102}$ have been used, where $\lambda$ represents the smallest occuring wavelength. The maximum time step $\Delta_{t}$ is, similarly as in Section VII A 1. determined by the Courant number, which leads for the FDTD scheme to the condition $v \Delta_{t}<\Delta_{z}$ [or $v \Delta_{t} \leq$ $\left(\Delta_{x}^{-2}+\Delta_{y}^{-2}+\Delta_{z}^{-2}\right)^{-1 / 2}$ for three spatial dimensions] ${ }^{52}$ Here, the velocity is obtained from the parameters in Eq. (44) as $v=\left(\mu_{0} \epsilon_{0} \epsilon_{\mathrm{r}}\right)^{-1 / 2}$. In related literature, choosing $v \Delta_{t}=\Delta_{z} / 2$ was found to be adequate.1021261

To reduce the numerical burden, different approaches using the pseudo-spectral time-domain method ${ }^{262}$ have been presented. ${ }^{154263}$ This method calculates the spatial derivatives using the fast Fourier transform in space. As long as Nyquist-Shannon theorem is not violated, the method is exact in space (and the introduced numerical dispersion minimal). However, the time derivative is still approximated with finite differences that cause numerical error and dispersion. Nevertheless, fewer spatial grid points are required to achieve reasonable accuracy (for example, the spatial discretization size $\Delta_{z}=\lambda / 10$ has been used $\left.{ }^{(154}\right)$. Thereby, the computational workload is reduced. These advantages come at the price of a more complicated implementation. In particular, absorbing boundary conditions must be implemented in order to avoid the wrap-around effect. Furthermore, sharp material parameter changes and the implementation of sources are not trivial anymore ${ }^{262}$

\section{Coupling Electric Field Updates and Density Matrix Updates}

Since the electric field in Maxwell's equations and the density matrix in the Bloch equations depend on each other, this coupling must be treated appropriately for any numerical method that solves Maxwell's equations. Bidégaray distinguishes between strongly and weakly coupled methods. ${ }^{257}$ The difference is the discretization of the density matrix in time and in relation to the electric field. Strongly coupled methods discretize the density matrix and the electric field at the same time value, weakly coupled methods apply a discretization which is staggered (a half time step difference between density matrix and elec- 


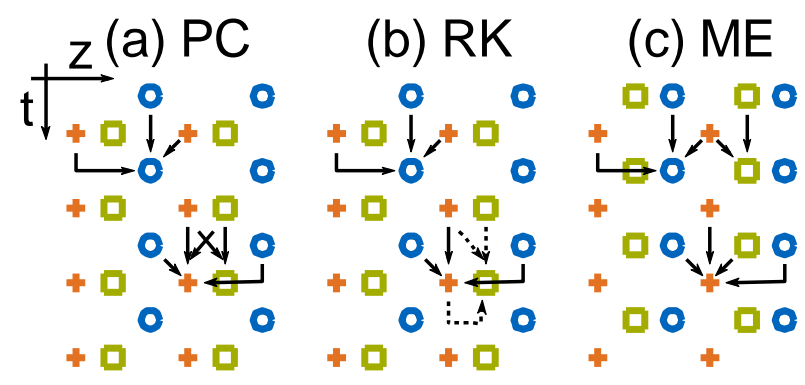

FIG. 17. Discretization and data dependencies of the finitedifference time-domain (FDTD) method combined with the (a) predictor-corrector (PC), (b) Runge-Kutta (RK), and (c) matrix exponential (ME) method for the density matrix updates. The crosses and circles denote the electric and magnetic fields, respectively, while the squares represent the density matrix. (a) The PC approach updates the electric field and the density matrix in parallel. (b) The RK method first updates the electric field (solid line) and then the density matrix (dashed line), where in the latter both the old and the new electric field values are used. (c) For the ME scheme, the updates of density matrix and the magnetic field are performed in parallel. The electric field discretization is shifted by a half time step.

tric field). In the following, we discuss various approaches to update the density matrix with different forms of coupling.

\section{Crank-Nicolson Scheme/Predictor-Corrector Method}

The pioneering work by Ziolkowski et al $[53[102$ treats the Bloch equations with the Crank-Nicolson scheme

$$
\hat{\rho}^{n+1}=\hat{\rho}^{n}+\frac{\Delta_{t}}{2}\left[\mathcal{F}^{n+1}\left(\hat{\rho}^{n+1}\right)+\mathcal{F}^{n}\left(\hat{\rho}^{n}\right)\right],
$$

where $n$ corresponds to the time $t_{n}=n \Delta_{t}, \Delta_{t}$ is the time step size, and $\mathcal{F}(\hat{\rho})=\mathcal{L}(\hat{\rho})+\mathcal{D}(\hat{\rho})$ represents the right hand side of the Lindblad equation (3). Since this implicit scheme requires solving a linear system of equations at every time step, usually modifications are employed to reduce the numerical load, such as keeping the field at a fixed value while advancing the density matrix by a time step ${ }^{207} \mathrm{~A}$ widely used variant is based on the predictorcorrector technique, where the update step first initializes $\hat{\rho}_{\mathrm{PC}}=\hat{\rho}_{n}$, then executes the procedure

$$
\hat{\rho}_{\mathrm{PC}} \leftarrow \hat{\rho}_{n}+\Delta_{t} \mathcal{F}\left(\frac{1}{2} \hat{\rho}_{\mathrm{PC}}+\frac{1}{2} \hat{\rho}_{n}\right)
$$

four times, and finally assigns the result to the value $\hat{\rho}_{n+1}=\hat{\rho}_{\mathrm{PC}} 53[102$

In Fig.17(a), the coupling of the method to the FDTD scheme is illustrated. It should be noted that this is a strongly coupled method and the electric field is updated with the same procedure (of course, $\mathcal{F}$ is replaced with the right hand side of Ampere's law) and in parallel to the density matrix update.

\section{Runge-Kutta Method}

Several research groups use the fourth-order Runge-Kutta (RK) method to solve the Bloch equations. $\frac{5516012391264}{4}$ As illustrated in Fig. 17(b), the method is strongly coupled since electric field and density matrix are discretized at the same time steps. The exact procedure is not always described in related work, but can be outlined as follows ${ }^{260}$ First, the electric field is updated using the standard FDTD update step. Then, the update of the density matrix using the rule

$$
\hat{\rho}^{n+1}=\hat{\rho}^{n}+\Delta_{t}\left(k_{1}+2 k_{2}+2 k_{3}+k_{4}\right) / 6
$$

follows, where $k_{1}=\mathcal{F}^{n}\left(\hat{\rho}^{n}\right), k_{2}=\mathcal{F}^{n+1 / 2}\left(\hat{\rho}^{n}+\Delta_{t} k_{1} / 2\right)$, $k_{3}=\mathcal{F}^{n+1 / 2}\left(\hat{\rho}^{n}+\Delta_{t} k_{2} / 2\right)$, and $k_{4}=\mathcal{F}^{n+1}\left(\hat{\rho}^{n}+\Delta_{t} k_{3}\right) \cdot \frac{265}{}$ Since the $\mathcal{F}^{n}$ contains the electric field $\mathbf{E}^{n}$ at time $t_{n}=$ $n \Delta_{t}$, not only the old and updated field values are required, but also the value at the half time step. The latter can be approximated by averaging between the old and the updated field value, i.e., $\mathbf{E}^{n+1 / 2} \approx\left(\mathbf{E}^{n}+\mathbf{E}^{n+1}\right) / 2$.

\section{Matrix Exponential Methods}

The methods of this group aim to solve the Bloch equations exactly for one time step. As illustrated in Fig.17(c), the updates of electric field and density matrix are weakly coupled, i.e., their updates are performed alternatingly. The density matrix update reads

$$
\hat{\rho}^{n+1 / 2}=\exp \left(\mathcal{F}^{n} \Delta_{t}\right) \hat{\rho}^{n-1 / 2},
$$

where $\mathcal{F}^{n}$ may depend on the electric field $\mathbf{E}^{n}$ and $\exp \left(\mathcal{F}^{n} \Delta_{t}\right)$ represents the exact solution of the Lindblad equation. After that, the standard FDTD update rule calculates $\mathbf{E}^{n+1}$ using $\mathbf{E}^{n}$ and $\hat{\rho}^{n+1 / 2}$.

If an analytical expression for the solution superoperator $\exp (\mathcal{F} t)$ exists, this method is clearly the most accurate one. However, finding such an analytical expression is far from trivial. In fact, the exact form of the exponential depends on the representation. In Liouville or coherence vector representation described in Section IIID the solution superoperator has the form $\exp (F t)$, where $F$ is a matrix. While this is straightforward to solve, the size of the matrix is in the order $N^{2} \times N^{2}$ for a $N \times N$ density matrix. Since the exponential of a $N \times N$ matrix would generally need $\mathcal{O}\left(N^{3}\right)$ operations, calculating the exponential in Liouville representation requires $\mathcal{O}\left(N^{6}\right)$ operations and becomes unfeasible for large $N$.

In regular representation, a solution for the Lindblad equation must be found first. The Strang splitting technique ${ }^{266}$ can help here to separate the effects of the Liouvillian $\mathcal{L}$ and the dissipation superoperator $\mathcal{D}$. The solution for the Liouvillian requires the calculation of $\exp \left(-\mathrm{i} \hbar^{-1} \hat{H} t\right)$, where the Hamiltonian $\hat{H}$ is a $N \times N$ Hermitian matrix. The calculation requires $\mathcal{O}\left(N^{3}\right)$ operations, which is still quite intensive. 
The Strang splitting introduces an additional error of $\mathcal{O}\left(\Delta_{t}^{2}\right)$ in general. Furthermore, $\mathcal{F}$ is generally time dependent due to its dependence on the time-varying electric field, in which case the resulting matrix exponentials contain an integral in the exponent. Commonly, the integral is approximated using the midpoint rule. This leads to the conclusion that in reality the accuracy of matrix exponential methods is comparable to other approaches. Nevertheless, this group of methods preserves certain matrix properties and despite their limited performance they have attracted the focus of many research groups.

Several techniques have been applied in order to improve the performance of matrix exponential methods. The already mentioned Strang splitting has not only been used to allow analytical solutions, but also to separate the time dependent and time independent part of $\mathcal{F}[154$ 256/257/263 This has the advantage that a part of the solution can be precalculated and applied at every time step, while for the remaining part efficient evaluation techniques exist in some cases. For example, we discovered that the coherence vector representation leads to a real skew-symmetric matrix in the exponential. This expression can be evaluated efficiently using the generalized Rodrigues' formula.267] Other techniques to calculate the matrix exponential ${ }^{268}$ have been applied in related work: An approximation based on the Cayley transform, 2561257/263 Magnus expansion via Sylvester's formula, ${ }^{[269}$ diagonalization of the matrix,, 270 the scaling and squaring method as well as a Krylov subspace method, 271 and Chebyshev polynomials. ${ }^{272}$

\section{Comparison of Numerical Methods for the Bloch Equations}

As already outlined above, the matrix exponential methods are the most computationally expensive ones. In fact, this was confirmed in a detailed investigation, 260 where both the Runge-Kutta and the predictor-corrector implementation outperformed the matrix exponential method. In this comparison, the predictor-corrector method demonstrated the best performance.

In terms of accuracy, Runge-Kutta methods have the highest order. However, the accuracy alone is not the crucial criterion. In particular, it was demonstrated that the Crank-Nicolson scheme does not preserve the positivity of the density matrix in the general case (at least when more than two energy levels are considered) and therefore might yield unrealistic results, e.g., negative populations. ${ }^{256}$ Furthermore, it was found that both the predictor-corrector and Runge-Kutta method yield negative populations in certain cases (e.g., long simulation end time combined with unfortunate choices for the time step size), while the matrix exponential method preserves the properties of the density matrix independent of the simulation settings. ${ }^{273}$

\section{Alternative Methods}

Besides the full-wave Bloch equations of the form Eq. (17), also related formalisms are used to model quantum systems interacting with a semiclassical optical field, requiring adapted numerical schemes which are often combined with the FDTD method for Maxwell's equations. For example, MB simulations which replace the Bloch equations by an equivalent evolution equation for the polarization vector, Eq. 72 , require modified schemes adapted to the second-order differential form of Eq. 722 .52159/274

If the dissipation term in the Bloch equations can be neglected and the quantum system is in a pure state, the time evolution can be described in a simplified manner with the time dependent Schrödinger equation, Eq. 11), for which suitable numerical schemes have been developed. ${ }^{275} \sqrt[279]{ }$ In analogy to the MB equations, the Schrödinger and Maxwell's equations can be combined to the Maxwell-Schrödinger approach, which is for example used to model nanoelectronic systems, $\frac{280 \mid 281}{1}$ or to describe the interaction of atoms with intense laser fields!282|283 As for the MB equations, such a coupled simulation complicates the numerical treatment, and various numerical schemes have been developed, e.g., combining FDTD or transmission line matrix simulations of Maxwell's equations with a spatial grid representation or eigenstate expansion of the wavefunction $\sqrt{280|281| 283}[286 \mathrm{In}$ this context, a recent interest has been on algorithms preserving the symplectic structure of the MaxwellSchrödinger equations, thus ensuring energy conservation of the coupled system. $284 \sqrt{286}$

\section{INCLUSION OF FURTHER EFFECTS}

\section{A. Local-Field Correction}

In principle, the current/polarization contribution of an individual quantum system at a given position can be directly represented in Maxwell's equations by a point source ${ }^{239}$ without using ensemble averaging as in Section IVA. However, the complexity of such an approach increases significantly with the number of quantum systems to be included $\stackrel{239}{ }$ Moreover, care must be taken that the field which drives the quantum system does not contain the divergent self-field contribution of the system itself, which further adds to the numerical load 239/287 $\mathrm{An}$ alternative approach, which is especially suitable for a large ensemble of quantum systems as considered in this paper, is based on macroscopic MB equations. Rather than setting up Bloch equations for each of the quantum systems, the ensemble is here modeled by representative density matrices $\rho_{i j}(\mathbf{x}, t)$ distributed over the device volume, e.g., placed on the spatial grid points, where $\mathbf{x}$ is the macroscopic position coordinate. Likewise, Maxwell's equations then contain the macroscopic current densities (see Section IV A and fields, defined as 


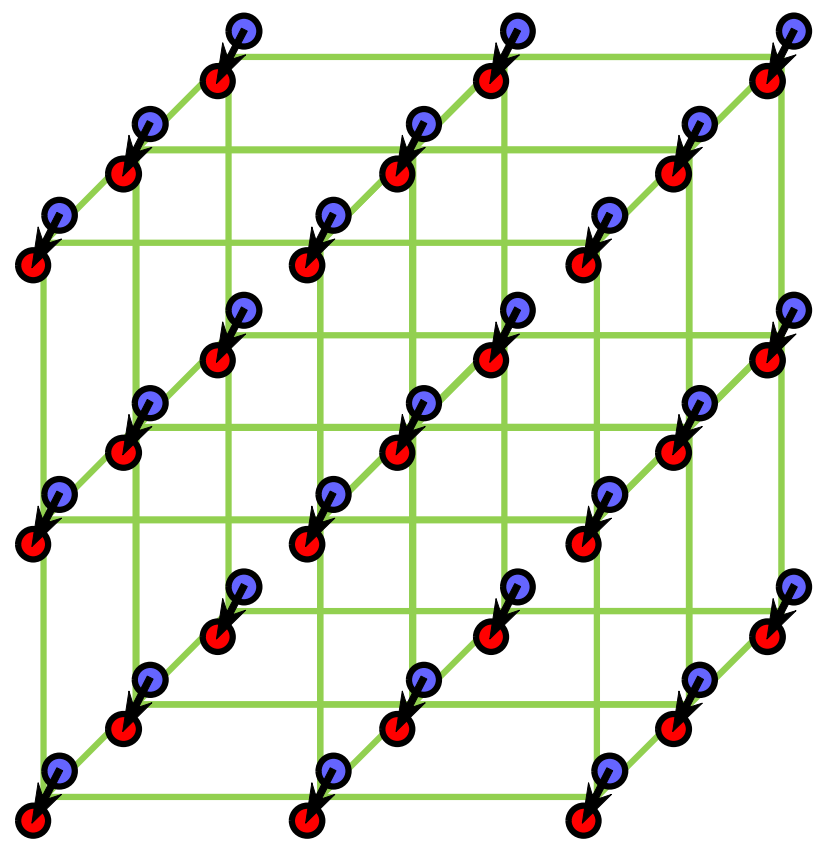

FIG. 18. Illustration of dipoles arranged in a cubic lattice.

ensemble averages over the individual microscopic contributions. From the macroscopic electric field $\mathbf{E}$ which is averaged over local variations associated with the individual dipoles, the local microscopic field which interacts with a given physical quantum system can be determined based on a compact Clausius-Mosotti type model. This local-field correction can for example lead to frequency shifts, and becomes relevant for densely spaced quantum systems as discussed in Section VIII A 1 ${ }^{631288}$ A related effect emerges in tightly localized artificial quantum systems consisting of multiple semiconductor atoms, see Section VIII A 2 ${ }^{289}$ It has been pointed out that local-field effects can be exploited as an additional design degree of freedom in nanostructures ${ }^{290}$

\section{Near-Dipole-Dipole Effects in Dense Media}

The macroscopic field $\mathbf{E}$ comprises contributions of external sources as well as an internal contribution $\mathbf{E}_{\mathrm{p}}$ due to the induced dipoles in the material, which is related to $\mathbf{P}_{\mathrm{q}}$. In the following, we consider a medium such as a gas or crystal lattice which consists of a dense collection of atoms, molecules or other quantum systems, as illustrated in Fig.18. The local field $\mathbf{E}_{\mathrm{L}}$ at the position of the considered quantum system is determined by replacing the volume-averaged field $\mathbf{E}_{\mathrm{p}}$ with the microscopic contribution $\mathbf{E}_{\text {near }}$ due to the nearby dipole moments, $\mathbf{E}_{\mathrm{L}}=\mathbf{E}-\mathbf{E}_{\mathrm{p}}+\mathbf{E}_{\text {near }}{ }^{185}$ Based on a microscopically large, but macroscopically small probe volume, which is conveniently chosen to be spherical, the macroscopic polarization contribution is obtained as $\mathbf{E}_{\mathrm{p}}=-\mathbf{P}_{\mathrm{q}} /\left(3 \epsilon_{0}\right)^{\frac{185}{185}}$ On the other hand, it can be shown that for dipoles arranged in a cubic lattice as illus-

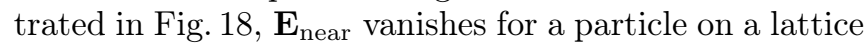
site, where the particle's self-field is not included $63[185$ This yields $\mathbf{E}_{\mathrm{L}}=\mathbf{E}+\mathbf{P}_{\mathrm{q}} /\left(3 \epsilon_{0}\right)$, which is also approximately true for other reasonably isotropic media and completely random arrangements, such as amorphous media or gases. ${ }^{63 \mid 185}$ Retardation effects are here negligible since the probe volume diameter is assumed to be much smaller than the optical wavelength ${ }^{63291}$ For two-level systems where the polarization is given by Eq. (71), the local-field corrections can thus be included in the Maxwell-Bloch equations by formally substituting $\Omega=\mathbf{d}_{12} \mathbf{E} / \hbar$ with

$$
\Omega_{\mathrm{L}}=\mathbf{d}_{12} \mathbf{E}_{\mathrm{L}} / \hbar=\Omega+2 \omega_{\mathrm{L}} \Re\left\{\rho_{21}\right\}
$$

in Eq. 67], 292 where the static Lorentz shift

$$
\omega_{\mathrm{L}}=d_{12}^{2} n_{3 \mathrm{D}} /\left(3 \hbar \epsilon_{0}\right)
$$

has the dimension of frequency. Here we have assumed a real-valued $\mathbf{d}_{12}$ for simplicity. Applying the RWA, we obtain Eq. 73 where we have to substitute $\underline{\hat{\Omega}}$ by

$$
\underline{\hat{\Omega}}_{\mathrm{L}}=\underline{\hat{\Omega}}+2 \omega_{\mathrm{L}} \eta_{21},
$$

which changes Eq. (73a) but not Eq. (73b) since there the local-field correction term cancels out

If the quantum systems are embedded in a host medium, such as dopant ions in a crystal, they interact not only with particles of the same species, but also with those of the host material, and the local-field correction must be suitably extended. Accordingly, above approach has been generalized to a dense collection of twolevel atoms embedded in a linear, potentially dispersive and absorptive host medium,, 293 and to multicomponent media in general ${ }^{294}$ Furthermore, above concept can be straightforwardly extended to more than two levels. ${ }^{295}$

We note that above correction to the MB equations has mainly been considered for ensembles of atoms or molecules, where typically much higher number densities are obtained than for artificial systems such as quantum dots. This model has enabled the analytica ${ }^{296}$ and numerical $297 \sqrt[299]{2 n v e s t i g a t i o n ~ o f ~ n u m e r o u s ~ e f f e c t s, ~ s u c h ~}$ as solitonic and ultrashort pulse propagation or optical switching. For artificial, tightly localized quantum systems, local-field effects are typically governed by the depolarization field, as discussed below.

\section{Depolarization Field in Tightly Localized Quantum Systems}

Artificial quantum systems, such as quantum dots, are nanostructures built from a larger number of semiconductor atoms. Thus, the quantum systems only "feel" an averaged polarization contribution of the individual atoms, which can be described by the background dielectric constant of the host material $\epsilon_{\mathrm{r}}$. Here, the local-field 


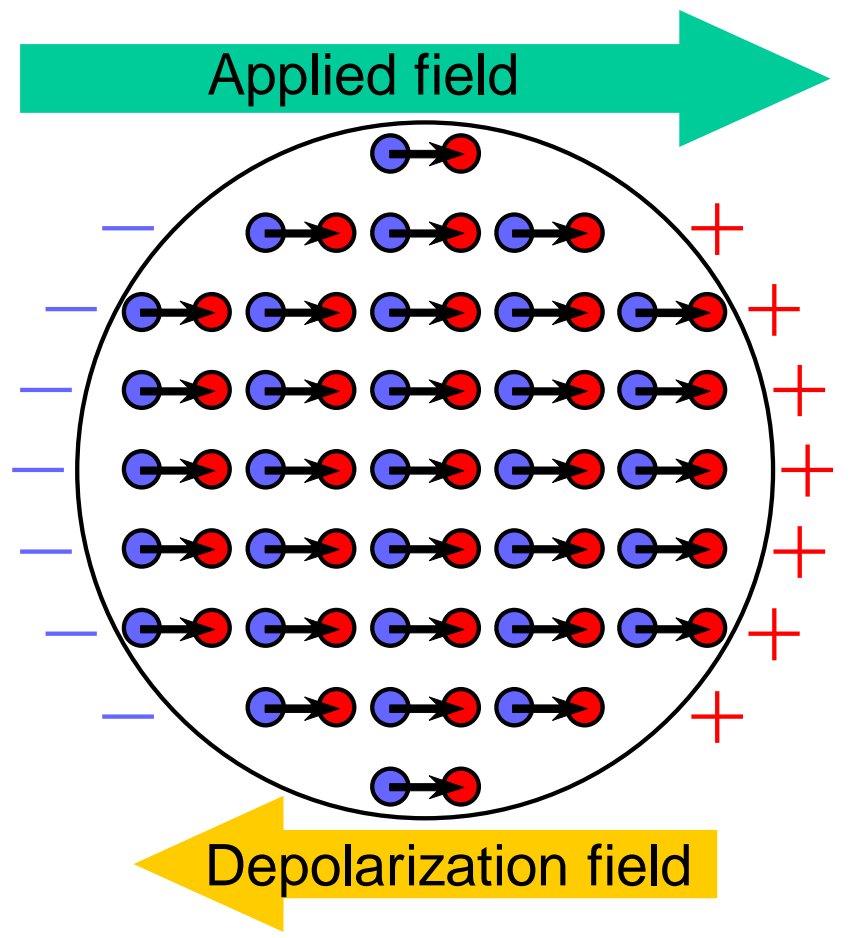

FIG. 19. Illustration of a localized polarizable object in an electric field.

correction accounts for the deviation of the mesoscopic average field inside the localized polarizable nanostructure from the macroscopic average field $\mathbf{E}$ in the entire composite material, which enters the macroscopic Maxwell equations. $\frac{290}{}$ In the following, we assume that $\epsilon_{\mathrm{r}}$ is real-valued and frequency independent, and is identical for the nanostructure and the surrounding material. As illustrated in Fig.19, the field $\mathbf{E}$ generates a polarization inside the polarizable object, and the uncompensated surface dipoles give rise to bound surface charges which induce an electric field in the object, the so-called depolarization field $\mathbf{E}_{\mathrm{d}}$, thus altering the local field inside to $\mathbf{E}_{\mathrm{L}}=\mathbf{E}+\mathbf{E}_{\mathrm{d}}$. We assume that the object's dimensions are much smaller than the wavelength of the exciting field. The (spatially averaged) depolarization field is then obtained as $\mathbf{E}_{\mathrm{d}}=-\mathbf{N P}_{\mathrm{q}} /\left(\epsilon_{0} \epsilon_{\mathrm{r}}\right)$, where $\mathbf{N}$ is the depolarization tensor which only depends on the geometry of the polarizable object $\frac{64}{6}$ For a localized two-level system of volume $V_{\mathrm{s}}$, the (volume-averaged) polarization is given by $\mathbf{P}_{\mathrm{q}}=\mathbf{d}_{12}\left(\rho_{12}+\rho_{21}\right) / V_{\mathrm{s}}$. Proceeding in the same way as in Section VIII A 1, we include the local-field correction into the MB equations by using Eq. (151) or Eq. 152), where we now have $e^{641289}$

$$
\omega_{\mathrm{L}}=-d_{12}^{2} L_{\mathrm{d}} /\left(\hbar \epsilon_{0} \epsilon_{\mathrm{r}} V_{\mathrm{s}}\right) .
$$

The depolarization factor $L_{\mathrm{d}}=\mathbf{d}_{12} \mathbf{N d}_{12} / d_{12}^{2}$ with $0 \leq$ $L_{\mathrm{d}} \leq 1$ accounts for the anisotropy of the object, such as an ellipsoidal quantum dot, and becomes $L_{\mathrm{d}}=1 / 3$ for spherical geometries 64 The resulting equations have been used for both analytical ${ }^{300}$ and numerical289/300|301 studies of local field effects in quantum dots.

\section{B. Inhomogeneous Broadening}

Homogeneous broadening is naturally considered in the Bloch equations. This can best be seen from the steady state solution in RWA, Eq. (128), yielding a Lorentzian lineshape with the width given by the dephasing rate of the corresponding transition, see Eqs. (129) and (132) as well as Fig.13(b). In addition, inhomogeneous broadening arises if the optically active medium consists of quantum systems with slightly different resonance frequencies $\frac{49 \mid 109}{{ }^{4}}$ as for example frequently arises in ensembles of quantum dots due to size fluctuations. Another example is Doppler broadening in a gas, 302 caused by the Doppler shift due to the thermal motion of the atoms or molecules. For a given transition between two states $|i\rangle$ and $|j\rangle$, the distribution of the resonance frequency $\omega_{i j}$ is commonly described by a distribution function $g_{i j}\left(\omega_{i j}-\bar{\omega}_{i j}\right)$ with $\int_{-\infty}^{\infty} g_{i j}(\omega) \mathrm{d} \omega=1$, where $\omega_{i j}-\bar{\omega}_{i j}$ is the deviation from the average resonance frequency $\bar{\omega}_{i j}$. For thermal Doppler broadening, $g_{i j}$ is given by a Gaussian distribution

$$
g_{i j}\left(\omega_{i j}-\bar{\omega}_{i j}\right)=\frac{1}{\sqrt{2 \pi} \sigma_{i j}} \exp \left[-\frac{\left(\omega_{i j}-\bar{\omega}_{i j}\right)^{2}}{2 \sigma_{i j}^{2}}\right] .
$$

In this case, the standard deviation becomes $\sigma_{i j}=$ $\left(\omega_{i j} / c\right)\left(k_{\mathrm{B}} T / m\right)^{1 / 2}$, where $k_{\mathrm{B}}$ is the Boltzmann constant, $T$ indicates the temperature, and $m$ is the mass of the atom or molecule ${ }^{302}$ The Gaussian distribution, Eq. (153), is also frequently used as a generic model if the distribution of resonance frequencies is not exactly known, e.g., to describe above mentioned inhomogeneous broadening in ensembles of quantum dots due to size fluctuations ${ }^{303}$ If the individual quantum systems contain more than one relevant optical transition with distributed resonance frequencies, in principle joint distribution functions have to be used.

Numerically, the full-wave or RWA Bloch equations, Eq. (17) or 43), have to be solved separately for each possible value of the resonance frequency (or each possible combination of resonance frequency values if the individual quantum systems contain more than one relevant optical transition) ${ }^{261}$ This requires discretizing the distribution function into a finite number of $N_{\text {inh }}$ bins with resonance frequencies $\omega_{i j}^{s}, s=1 . . N_{\mathrm{inh}}$. Each of these bins is represented by a corresponding quantum system subensemble with density matrix $\rho_{i j}^{s}$, where the fraction of carriers $p_{s}$ is proportional to the weight of that bin and $\sum_{s} p_{s}=1$. The polarization current density is then obtained from a generalized version of Eq. 48, ,

$$
\mathbf{J}_{\mathrm{q}}=n_{3 \mathrm{D}} \sum_{i, j} \mathbf{d}_{j i} \sum_{s} p_{s} \partial_{t} \rho_{i j}^{s} .
$$

In SVAA, the polarization amplitude is given by a gen- 
eralized form of Eq. 64,

$$
\underline{\mathbf{P}}=2 n_{3 \mathrm{D}} \sum_{\omega_{i j}>0} \mathbf{d}_{j i} \sum_{s} p_{s} \eta_{i j}^{s} .
$$

For certain broadening mechanisms, such as fluctuations in quantum dot size, also $\mathbf{d}_{i j}$ can in principle vary which could be considered by introducing a quantity $\mathbf{d}_{i j}^{s}$ in Eqs. (154) and (155) in analogy to $\omega_{i j}^{s}$, but this effect is usually neglected.

In certain cases, inhomogeneous broadening can also be considered in analytical solutions of the MB equations based on the RWA. These usually invoke the factorization ansatz, which assumes that non-resonant systems with a finite frequency detuning $\Delta=\omega_{\mathrm{c}}-\omega_{21} \neq 0$ essentially respond in the same way to the optical field as the resonant ones, apart from a detuning dependent amplitude $F(\Delta) \stackrel{49}{\square}$ In Section VI A 2, this approach has been demonstrated in the context of self-induced transparency.

\section{Noise}

Noise in optoelectronic devices arises for example from spontaneous emission and from processes in the semiconductor host, such as lattice vibrations. Noise and fluctuations can generally be included into the semiclassical MB equations by adding stochastic terms. $\frac{304 / 305}{13}$ Numerically, the stochastic terms are typically implemented by using a pseudorandom number generator to obtain uncorrelated, Gaussian distributed random numbers for every grid point ${ }^{173 / 306}$ The MB equations, complemented by these additional stochastic terms, are then numerically solved as discussed in Section VII] for example with an FDTD-based approach! ${ }^{[73 \mid 306}$ The stochastic terms are systematically obtained from quantum Langevin equations, ${ }^{171 / 307}$ which are then represented by equivalent stochastic c-number equations, $\frac{173[308 \mid 309}{1 . e .,}$ evolution equations for operator expectation values with additional stochastic terms.

Spontaneous emission obviously plays an important role in optoelectronic devices. While the resulting recombination can simply be included by nonlinear rate terms for the carrier occupations in Eq. 17b or $43 \mathrm{~b}$,, 3031310 the noise contribution is not included in the $\mathrm{MB}$ model due to its semiclassical nature. This effect can however be considered in terms of a Gaussian white noise source in the optical propagation equation. ${ }^{173 / 311 / 312}$ In a different model, also dipole fluctuations are included by adding Langevin noise terms not only to the propagation equation, but also to Eq. (17a) for the off-diagonal den-

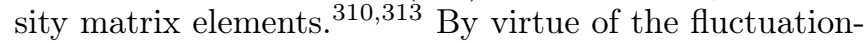
dissipation theorem, a decay of populations, coherences or the optical field is generally accompanied by fluctuations, and an MB equation model which includes such decay-induced fluctuations has been presented $\left[\frac{56 / 306}{}\right.$ Furthermore, an extension of the stochastic c-number approach to incorporate nonclassical effects has been discussed 309

\section{APPLICATION TO OPTOELECTRONIC DEVICES}

\section{A. Bulk and Quantum Well Interband Optoelectronic Devices}

For interband optoelectronic devices based on semiconductor bulk or quantum-well media, the conduction and valence band states are given by $|\mathrm{c}, \mathbf{k}\rangle$ and $|\mathrm{v}, \mathbf{k}\rangle$, respectively. Here, $\mathbf{k}$ is the three-dimensional crystal wavevector in bulk media or the two-dimensional in-plane wavevector for quantum well structures, see Section III C. In the following, we define $\rho_{\mathrm{c}, \mathbf{k}}$ and $\left(1-\rho_{\mathrm{v}, \mathbf{k}}\right)$ as the electron occupation probability of a conduction band state $|\mathrm{c}, \mathbf{k}\rangle$ and a valence band state $|\mathrm{v}, \mathbf{k}\rangle$, respectively, i.e., $\rho_{\mathrm{v}, \mathbf{k}}$ corresponds to the hole occupation probability of a valence band state. Restricting ourselves to direct bandgap semiconductors in a two-band approximation and using that the optical transitions are $\mathbf{k}$ conserving, as can for quantum wells be seen from Eq. 24$), \frac{65}{6}$ the Bloch equations, Eq. (17), become

$$
\begin{aligned}
\partial_{t} \rho_{\mathrm{cv}, \mathbf{k}}= & -\mathrm{i} \omega_{\mathrm{cv}, \mathbf{k}} \rho_{\mathrm{cv}, \mathbf{k}}-\mathrm{i} \Omega_{\mathbf{k}}\left(\rho_{\mathrm{c}, \mathbf{k}}+\rho_{\mathrm{v}, \mathbf{k}}-1\right) \\
& +\left[\partial_{t} \rho_{\mathrm{cv}, \mathbf{k}}\right]_{\mathrm{col}}, \\
\partial_{t} \rho_{\alpha, \mathbf{k}}= & \mathrm{i}\left(\Omega_{\mathbf{k}} \rho_{\mathrm{cv}, \mathbf{k}}^{*}-\Omega_{\mathbf{k}}^{*} \rho_{\mathrm{cv}, \mathbf{k}}\right)+\left[\partial_{t} \rho_{\alpha, \mathbf{k}}\right]_{\mathrm{col}},
\end{aligned}
$$

with $\alpha=\mathrm{c}$, v. Here, the sum over $\mathbf{k}$ implicitly also includes summation over the two possible spin orientations. This equation applies to quantum wells, where the carriers are treated as a two-dimensional gas, as well as bulk media. The dissipation processes are here included by general collision terms $\left[\partial_{t} \rho_{\mathrm{cv}, \mathbf{k}}\right]_{\mathrm{col}}$ and $\left[\partial_{t} \rho_{\alpha, \mathbf{k}}\right]_{\mathrm{col}}$. These can be modeled on a microscopic level, in particular accounting for carrier-carrier and carrier-phonon scattering, or under certain approximations by relaxation rate terms similar to those in Eq. 17] . ${ }^{65 \mid 66}$ Many-body Coulomb interactions can be taken into account based on the Hartree-Fock approximation, which results in the so-called semiconductor Bloch equations, which have the form of Eq. (156) but feature renormalized transition and Rabi frequencies

$$
\begin{aligned}
\omega_{\mathrm{cv}, \mathbf{k}} & =\frac{1}{\hbar} E_{\mathrm{cv}, \mathbf{k}}-\frac{1}{\hbar} \sum_{\mathbf{q} \neq \mathbf{k}} V_{|\mathbf{k}-\mathbf{q}|}\left(\rho_{\mathrm{c}, \mathbf{q}}+\rho_{\mathrm{v}, \mathbf{q}}\right), \\
\Omega_{\mathbf{k}} & =\frac{\mathbf{d}_{\mathrm{cv}, \mathbf{k}} \mathbf{E}}{\hbar}+\frac{1}{\hbar} \sum_{\mathbf{q} \neq \mathbf{k}} V_{|\mathbf{k}-\mathbf{q}|} \rho_{\mathrm{cv}, \mathbf{q}},
\end{aligned}
$$

leading to a coupling of the states with different $\mathbf{k}$ through the time dependent renormalization

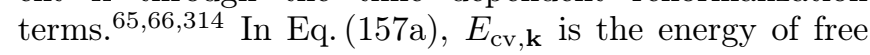
electron-hole pairs, which can in a simple model be described as

$$
E_{\mathrm{cv}, \mathbf{k}}=\frac{1}{2} \hbar^{2} \mathbf{k}^{2}\left(\frac{1}{m_{\mathrm{e}}^{*}}+\frac{1}{m_{\mathrm{h}}^{*}}\right)+E_{\mathrm{g}} .
$$

Here, $E_{\mathrm{g}}$ denotes the band gap energy, or for quantum wells the energy difference between the electron and hole state energies. Furthermore, $m_{\mathrm{e}}^{*}$ and $m_{\mathrm{h}}^{*}$ are the electron 
and hole effective masses, assuming a parabolic dispersion relation near the conduction and valence band edges, respectively. $V_{\mathbf{q}}$ is the Coulomb potential in Fourier representation, which is in a bulk with probe volume $V_{\mathrm{p}}$ and background permittivity $\epsilon_{\mathrm{r}}$ given by $V_{q}=e^{2} /\left(\epsilon_{0} \epsilon_{\mathrm{r}} V_{\mathrm{p}} q^{2}\right)$, and in a quantum well structure with in-plane cross section $S$ by $V_{q}=e^{2} /\left(2 \epsilon_{0} \epsilon_{\mathrm{r}} q S\right)$. Screening effects, which result from the response of the other carriers and weaken the potential, are then incorporated as corrections to the Hartree-Fock equations in the form of a modified $V_{q} \cdot{ }^{[6566}$ Summing in Eq. (48) over the initial and final states $|i\rangle=|\mathrm{c}, \mathbf{k}\rangle$ and $|j\rangle=|\mathrm{v}, \mathbf{q}\rangle$ where we consider that the total number density of electron-hole pairs is given by

$$
n_{\mathrm{cv}}(t)=V_{\mathrm{p}}^{-1} \sum_{\mathbf{k}} \rho_{\mathrm{c}, \mathbf{k}}(t)=V_{\mathrm{p}}^{-1} \sum_{\mathbf{k}} \rho_{\mathrm{v}, \mathbf{k}}(t),
$$

and using the $\mathbf{k}$ conservation of optical transitions, $\mathbf{d}_{i j}=$ $\mathbf{d}_{\mathrm{cv}, \mathbf{k}} \delta_{\mathbf{k}, \mathbf{q}}$, we obtain for the polarization term

$$
\partial_{t} \mathbf{P}_{\mathrm{q}}=V_{\mathrm{p}}^{-1} \sum_{\mathbf{k}}\left(\mathbf{d}_{\mathrm{cv}, \mathbf{k}} \partial_{t} \rho_{\mathrm{vc}, \mathbf{k}}+\text { c.c. }\right) .
$$

With Eq. 160), Eqs. 156 and 157 can be coupled to the Maxwell equations, Eq. (44), resulting in the semiconductor Maxwell-Bloch equations. These equations have been extensively used for the simulation of semiconductor lasers and related devices. $\frac{\square 1812031315}{\text { Further- }}$ more, they have been adapted to the modeling of quantum wire structures, 316 as well as graphene ${ }^{319}$ and carbon nanotubes $\sqrt[320]{ }$

As stated in Section [1] a further discussion of this model is beyond the scope of this paper. Rather, we will focus here on approaches which reduce above twoband model to macroscopic two- or $N$-level Bloch equations. As a first step, the collision terms in Eq. (156) are modeled by relaxation rate terms similar to those in Eq. 17] $\frac{65}{68}$ Extending the rate equation model Eq. (9) to states $|\alpha, \mathbf{k}\rangle$ results in a Boltzmann-type collision term for the populations, ${ }^{[65}$ which can in consideration of Pauli blocking be written as

$$
\begin{aligned}
{\left[\partial_{t} \rho_{\alpha, \mathbf{k}}\right]_{\mathrm{col}}=} & -\rho_{\alpha, \mathbf{k}}\left(W_{\alpha \beta, \mathbf{k}}^{\alpha}+W_{\alpha \alpha, \mathbf{k}}^{\alpha}\right) \\
& +\left(1-\rho_{\alpha, \mathbf{k}}\right)\left(W_{\alpha \beta, \mathbf{k}}^{\beta}+W_{\alpha \alpha, \mathbf{k}}^{\beta}\right) .
\end{aligned}
$$

Here, $\beta \neq \alpha$, i.e., for the conduction band collision term $(\alpha=\mathrm{c})$ we have $\beta=\mathrm{v}$, while $\beta=\mathrm{c}$ for $\alpha=\mathrm{v}$. The Boltzmann rates are related to the electron transition rates of Section II B 1 by $W_{\alpha \alpha^{\prime}, \mathbf{k}}^{\mathrm{c}}=\sum_{\mathbf{k}^{\prime}} r_{\alpha, \mathbf{k} \rightarrow \alpha^{\prime}, \mathbf{k}^{\prime}}\left(1-f_{\alpha^{\prime}, \mathbf{k}^{\prime}}\right)$ and $W_{\alpha \alpha^{\prime}, \mathbf{k}}^{\mathrm{v}}=\sum_{\mathbf{k}^{\prime}} r_{\alpha^{\prime}, \mathbf{k}^{\prime} \rightarrow \alpha, \mathbf{k}} f_{\alpha^{\prime}, \mathbf{k}^{\prime}}$, where $f_{\alpha, \mathbf{k}}$ denotes the electron occupation probability, i.e., $f_{\mathrm{c}, \mathbf{k}}=$ $\rho_{\mathrm{c}, \mathbf{k}}$ and $f_{\mathrm{v}, \mathbf{k}}=1-\rho_{\mathrm{v}, \mathbf{k}}$. For example, spontaneous emission and carrier-phonon scattering can be modeled by Eq. 161) with adequately chosen transition rates $r_{\alpha, \mathbf{k} \rightarrow \alpha^{\prime}, \mathbf{k}^{\prime}}$, while the inclusion of carrier-carrier interactions beyond Hartree-Fock effectively requires rates which themselves depend on the carrier distribution. ${ }^{65}$
Furthermore modeling the dephasing in analogy to Eq. 12, we obtain

$$
\begin{aligned}
{\left[\partial_{t} \rho_{\mathrm{cv}, \mathbf{k}}\right]_{\mathrm{col}} } & =-\gamma_{\mathrm{cv}, \mathbf{k}} \rho_{\mathrm{cv}, \mathbf{k}}, \\
{\left[\partial_{t} \rho_{\alpha, \mathbf{k}}\right]_{\mathrm{col}} } & =\Gamma_{\beta \alpha, \mathbf{k}}-r_{\alpha \beta, \mathbf{k}} \rho_{\alpha, \mathbf{k}}+\Gamma_{\alpha \alpha, \mathbf{k}}-r_{\alpha \alpha, \mathbf{k}} \rho_{\alpha, \mathbf{k}} .
\end{aligned}
$$

In Eq. 162a), $\gamma_{\mathrm{cv}, \mathbf{k}}$ indicates the dephasing rate. Rearranging the contributions in Eq. (161), Eq. 162b) is obtained, where the first two terms on the right hand side with $\beta \neq \alpha$ describe interband processes, while the other two terms model the intraband transitions. Here, $r_{\alpha \alpha^{\prime}, \mathbf{k}}=W_{\alpha \alpha^{\prime}, \mathbf{k}}^{\alpha}$ denotes the interband (for $\alpha^{\prime}=\beta$ ) or intraband (for $\alpha^{\prime}=\alpha$ ) recombination rate due to nonradiative transitions and spontaneous emission. Furthermore, $\Gamma_{\alpha^{\prime} \alpha, \mathbf{k}}=\left(1-\rho_{\alpha, \mathbf{k}}\right) W_{\alpha \alpha^{\prime}, \mathbf{k}}^{\beta}$ describes the filling of state $|\alpha, \mathbf{k}\rangle$, and can for $\alpha^{\prime}=\beta$ be interpreted as a pump rate, where carriers are induced for example by an injection current or optical pumping. Summation of Eq. 161) or Eq. $162 \mathrm{~b}$ over $\mathbf{k}$ yields $\sum_{\mathbf{k}}\left[\partial_{t} \rho_{\mathrm{c}, \mathbf{k}}\right]_{\mathrm{col}}=\sum_{\mathbf{k}}\left[\partial_{t} \rho_{\mathrm{v}, \mathbf{k}}\right]_{\mathrm{col}}$, as expected from Eq. 159). In more detail, for the intraband contributions we have

$$
\sum_{\mathbf{k}}\left(\Gamma_{\alpha \alpha, \mathbf{k}}-r_{\alpha \alpha, \mathbf{k}} \rho_{\alpha, \mathbf{k}}\right)=0,
$$

while the interband terms fulfill

$$
\begin{aligned}
\sum_{\mathbf{k}} \Gamma_{\mathrm{vc}, \mathbf{k}} & =\sum_{\mathbf{k}} \Gamma_{\mathrm{cv}, \mathbf{k}}, \\
\sum_{\mathbf{k}} r_{\mathrm{cv}, \mathbf{k}} \rho_{\mathrm{c}, \mathbf{k}} & =\sum_{\mathbf{k}} r_{\mathrm{vc}, \mathbf{k}} \rho_{\mathrm{v}, \mathbf{k}} .
\end{aligned}
$$

To obtain compact two-level Bloch equations, we assume a $\mathbf{k}$ independent dipole matrix element $\mathbf{d}_{\mathrm{cv}, \mathbf{k}}=\mathbf{d}_{21}$ in Eq. 157b and ignore the renormalization contribution, yielding the usual definition $\Omega_{\mathbf{k}}=\hbar^{-1} \mathbf{d}_{21} \mathbf{E}$. Summing Eq. 156b over $\mathbf{k}$ yields with Eqs. 1159), 162b), 163) and (164) and $P_{\mathrm{cv}}=V_{\mathrm{p}}^{-1} \sum_{\mathrm{k}} \rho_{\mathrm{cv}, \mathbf{k}}$

$$
\partial_{t} n_{\mathrm{cv}}=\mathrm{i}\left(\Omega P_{\mathrm{cv}}^{*}-\Omega^{*} P_{\mathrm{cv}}\right)+\Gamma_{12}-\gamma_{1} n_{\mathrm{cv}} .
$$

For electrical pumping, the injection rate $\Gamma_{12}=$ $\sum_{\mathbf{k}} \Gamma_{\beta \alpha, \mathbf{k}}$ (with $\alpha=\mathrm{c}, \beta=\mathrm{v}$ or $\alpha=\mathrm{v}, \beta=\mathrm{c}$ ) can be modeled as $\Gamma_{12}=\eta V_{\mathrm{p}}^{-1} I / e$, where $\eta$ and $I$ denote the injection efficiency and current, respectively. The recombination rate $\gamma_{1}$, which includes nonradiative and spontaneous transitions, is obtained by averaging over the carrier distribution, $\gamma_{1}=\sum_{\mathbf{k}} r_{\alpha \beta, \mathbf{k}} \rho_{\alpha, \mathbf{k}} / n_{\mathrm{cv}}$. Proceeding in a similar manner for Eq. (156a) by neglecting the $\mathbf{k}$ dependence of $\omega_{\mathrm{cv}, \mathbf{k}}$ is not feasible, due to the problems arising from the summation over $\left(\rho_{\mathrm{c}, \mathbf{k}}+\rho_{\mathrm{v}, \mathbf{k}}-1\right)$. Various strategies have been developed to circumvent this problem $[67[69]$ Here we follow the approach by Yao et al., $\frac{68}{6}$ formulated in the framework of the RWA. Thus, we start by introducing the slowly varying field envelope $\underline{E}$ and the transformed off-diagonal elements defined in Eqs. (41) and (42), respectively. Furthermore assuming a 
k independent Rabi frequency $\underline{\Omega}=\hbar^{-1} \mathbf{d}_{\mathrm{cv}} \underline{\mathbf{E}}$, Eq. 156a yields with Eq. (162a)

$$
\partial_{t} \eta_{\mathrm{cv}, \mathbf{k}}=\left(\mathrm{i} \Delta_{\mathbf{k}}-\gamma_{\mathrm{cv}, \mathbf{k}}\right) \eta_{\mathrm{cv}, \mathbf{k}}-\frac{\mathrm{i}}{2} \underline{\Omega}\left(\rho_{\mathrm{c}, \mathbf{k}}+\rho_{\mathrm{v}, \mathbf{k}}-1\right),
$$

where $\Delta_{\mathbf{k}}=\omega_{\mathrm{c}}-\omega_{\mathrm{cv}, \mathbf{k}}$ with $\omega_{\mathrm{cv}, \mathbf{k}}$ given by Eq. 1157a). In the framework of semi-phenomenological macroscopic MB equation models, the renormalization term is often neglected $\frac{6869}{\text { The }} \mathbf{k}$ dependence can be modeled with Eq. (158) or a more sophisticated description. Dividing Eq. (166) by $\left(\mathrm{i} \Delta_{\mathbf{k}}-\gamma_{\mathrm{cv}, \mathbf{k}}\right)$, summing over $\mathbf{k}$ and defining $p_{\mathrm{cv}}=V_{\mathrm{p}}^{-1} \sum_{\mathrm{k}} \eta_{\mathrm{cv}, \mathbf{k}}$, we obtain

$$
\partial_{t} p_{\mathrm{cv}}=-\frac{\mathrm{i}}{2} \frac{\tau_{1}}{\tau_{2}} \underline{\Omega} n_{\mathrm{cv}}-\tau_{2}^{-1} p_{\mathrm{cv}}
$$

where we have furthermore used Eq. (159) and introduced the complex parameters $\tau_{1}$ and $\tau_{2}$ with

$$
\begin{gathered}
V_{\mathrm{p}}^{-1} \sum_{\mathbf{k}} \frac{\rho_{\mathrm{c}, \mathbf{k}}+\rho_{\mathrm{v}, \mathbf{k}}-1}{\gamma_{\mathrm{cv}, \mathbf{k}}-\mathrm{i} \Delta_{\mathbf{k}}}=\tau_{1} n_{\mathrm{cv}}, \\
V_{\mathrm{p}}^{-1} \sum_{\mathbf{k}} \frac{\eta_{\mathrm{cv}, \mathbf{k}}}{\gamma_{\mathrm{cv}, \mathbf{k}}-\mathrm{i} \Delta_{\mathbf{k}}}=\tau_{2} p_{\mathrm{cv}} .
\end{gathered}
$$

Equation (167) and Eq. 165 in RWA,

$$
\partial_{t} n_{\mathrm{cv}}=\frac{\mathrm{i}}{2}\left(\underline{\Omega} p_{\mathrm{cv}}^{*}-\underline{\Omega}^{*} p_{\mathrm{cv}}\right)+\Gamma_{12}-\gamma_{1} n_{\mathrm{cv}},
$$

constitute macroscopic Bloch equations for interband transitions in bulk semiconductor and quantum well systems. In order to obtain a Maxwell-Bloch model, Eqs. (167) and (169) can be coupled to the optical propagation equation in SVAA, Eq. 62 , where the RWA polarization term is obtained from Eq. 75 as $\underline{\mathbf{P}}=2 \mathbf{d}_{\mathrm{cv}} p_{\mathrm{cv}}$. The parameters $\tau_{1}$ and $\tau_{2}$ introduced in Eq. (168) can for example be evaluated numerically, or by fitting to experimental data ${ }^{68}$ In this context, it has been found that $\tau_{1}$ and $\tau_{2}$ can be treated as independent of the optical intensity, but that especially $\tau_{2}$ shows a pronounced dependence on $n_{\mathrm{cv}}$ which should be considered in the model, $\stackrel{68}{6}$ and also allows a phenomenological reintroduction of renormalization effects.

\section{B. Quantum Well Intersubband Devices}

Intersubband devices, such as QCLs ${ }^{7}$ quantum cascade detectors ${ }^{819}$ and quantum well infrared photodetectors (QWIPs) 10 commonly utilize optical intersubband transitions between quantized energy levels in the conduction band $\Gamma$ valley of a multiple quantum well structure. The Maxwell-Bloch model has been extensively applied to such devices, especially for the dynamic modeling of QCLs. The quantized states $\left|\psi_{i}, \mathbf{k}\right\rangle$, also referred to as subbands, are characterized by their wavefunction $\psi_{i}(x)$ where $i$ is the subband index, and inplane wavevector $\mathbf{k}=\left[k_{y}, k_{z}\right]^{\mathrm{T}}$. These states are commonly found by solving the one-dimensional effective mass Schrödinger equation, obtained from inserting the ansatz Eq. (19) into Eq. 201, for the quantum well potential $V(z)$. As mentioned in Section IIIC 1, band bending due to space charge effects is usually considered by solving the coupled Schrödinger-Poisson equation system,,$\frac{1251126}{1}$ and also nonparabolicity effects, which play a role especially in mid-infrared devices, can be included $127 / 128$ These calculations yield the eigenenergies $E_{i}$ and wavefunctions $\psi_{i}$, and thus the transition frequencies $\omega_{i j}=\hbar^{-1}\left(E_{i}-E_{j}\right)$ and the dipole matrix elements, Eq. 25], as input for the Bloch equations. We again choose the semiconductor Bloch equations as a starting point, with a form analogous to Eq. 156). Due to the typically low doping levels of QCLs, the Hartree-Fock renormalization effects in Eq. 157) have been found to be relatively small,, 321 and also Pauli blocking only plays a secondary role. Furthermore, we can assume $\mathbf{k}$ independent transition frequencies $\omega_{i j}$ at least for terahertz QCLs, where the energetic level spacings are smaller than in mid-infrared QCLs and thus the subbands have nearly parallel dispersion relationships. Under these assumptions, summing over $\mathbf{k}$ yields Bloch equations of the form Eq. (17), where we have used that the dipole matrix element is $\mathbf{k}$ conserving, and introduced intersubband scattering rates $r_{j \rightarrow i}$ which are related to the generally $\mathbf{k}$ dependent rates $r_{j, \mathbf{q} \rightarrow i, \mathbf{k}}$ by $r_{j \rightarrow i} \rho_{j j}=\sum_{\mathbf{k}, \mathbf{q}} r_{j, \mathbf{q} \rightarrow i, \mathbf{k}} \rho_{j j, \mathbf{q}}$. Assuming either moderate temporal variations of the intrasubband electron distribution $\rho_{j j, \mathbf{k}}$ or a moderate $\mathbf{k}$ dependence of the rates, the $r_{j \rightarrow i}$ are approximately given by

$$
r_{j \rightarrow i}=\sum_{\mathbf{k}, \mathbf{q}} r_{j, \mathbf{q} \rightarrow i, \mathbf{k}} \rho_{j j, \mathbf{q}}^{0} / \sum_{\mathbf{k}} \rho_{j j, \mathbf{k}}^{0} .
$$

Here, $\rho_{j j, \mathbf{k}}^{0}$ describes the steady state electron distribution in the subband ${ }^{322}$ Notably, the intrasubband electron distributions in QCLs can often be reasonably well approximated by Fermi-Dirac or MaxwellBoltzmann distributions, parametrized by subband electron temperatures which can significantly exceed the lattice temperatures ${ }^{1191323}$ By contrast, the off-diagonal density matrix elements generally vary strongly with time, and no clearly defined concept exists how the $\mathbf{k}$ averaging should be performed to obtain an effective dephasing rate $\gamma_{i j}$ from a relaxation term of the form Eq. 162a), $\left[\partial_{t} \rho_{i j, \mathbf{k}}\right]_{\mathrm{col}}=-\gamma_{i j, \mathbf{k}} \rho_{i j, \mathbf{k}}$. This especially matters if the ratio $\rho_{i i, \mathbf{k}}^{0} / \rho_{j j, \mathbf{k}}^{0}$ has a strong $\mathbf{k}$ dependence, as is the case for significantly different subband electron temperatures or highly non-thermal distributions ${ }^{322}$ Often, an average over the population inversion of the involved subbands is applied, 1011324

$$
\gamma_{i j}=\sum_{\mathbf{k}} \gamma_{i j, \mathbf{k}}\left|\rho_{i i, \mathbf{k}}^{0}-\rho_{j j, \mathbf{k}}^{0}\right| / \sum_{\mathbf{k}}\left|\rho_{i i, \mathbf{k}}^{0}-\rho_{j j, \mathbf{k}}^{0}\right|,
$$

and a comparison to an alternative way of averaging has yielded similar results for terahertz QCLs ${ }^{98}$ Apart from very few exceptions based on the full-wave MB equations,$\frac{[61|106| 325}{,}$ usually the MB equations in RWA 
and SVAA, Eqs. 124 and 43), are used for the modeling of QCLs and related devices. Also, apart from some cases including multiple subbands, 18 [62 97|122|322|326 typically a two-level model is employed. In the case of midinfrared QCLs, where nonparabolicity effects play a more important role, an approach similar to Eq. 167) can be envisioned. The transition and dephasing rates are usually empirically chosen, or extracted from fits to experimental data. Alternatively, they can be calculated from Eqs. 170 and (171) based on the Hamiltonians of the relevant scattering mechanisms, such as electron-electron interactions, scattering with acoustic and longitudinal optical phonons, as well as impurity, interface roughness and alloy scattering! $119 \sqrt[322]{3}$ Here, the use of dissipation rates derived from steady-state models is consistent with the Markovian and time-homogeneous character of the Lindblad dissipator, which provides the basis for the Bloch equations. The corresponding scattering rates are typically evaluated based on Fermi's golden rule, ${ }^{[119}$ while the associated pure dephasing rates can be obtained from Ando's model. $98|327| 328$

For QCLs, the MB equations have primarily been used to study ultrashort pulse generation by modelocking $30|120| 122|232| 233|326| 329] 332$ and the closely related formation of coherent instabilities, $\frac{202|208| 333 \mid}{\text { as well as }}$ the generation of frequency combs $\frac{62|97| 322|334| 335 \mid}{1 n}$ detail, it has been found that coherent multimode instabilities result in the emergence of sidebands around the original longitudinal mode, giving rise to broadband multimode operation. 202 208 Furthermore, active mode-locking has been investigated where short pulses are generated by modulating the laser current at the cavity roundtrip frequency, yielding good agreement between simulations and measurements. 329 A32] Also the possibility of realizing passive mode-locking in QCLs has been theoretically explored! $30|120| 122|232| 233$ Here, pulse formation is obtained by adding saturable absorption regions, where SIT mode-locking, discussed in Section VIA2 constitutes a special variant. Besides, frequency comb operation has been studied, where an equidistant line spectrum is generated, which serves as a ruler in the frequency domain for spectroscopic and sensing applications. Here, a perturbative treatment of the MB equations imposing a comb-like spectrum has been employed, $234 \sqrt[335]{\text { as well }}$ as full numerical simulations. ${ }^{62 / 97 / 322}$ In most of above works, spatial hole burning has been considered based on Eqs. 123 and (124), as it considerably affects the QCL dynamics. In addition, various other effects have been implemented which can play an important role for modelocked and frequency comb operation in QCLs, such as tunneling across thick barriers ${ }^{62|97| 322}$ and group velocity dispersion due to the waveguide and bulk semiconductor material. $62|97| 233|322| 335|336|$ For optical excitation on very short timescales, memory effects become important and the presuppositions of the Lindblad approach are too restrictive, requiring the use of more complex models such as the quantum-kinetic schemes. 337 [339

As mentioned above, MB simulations have for example

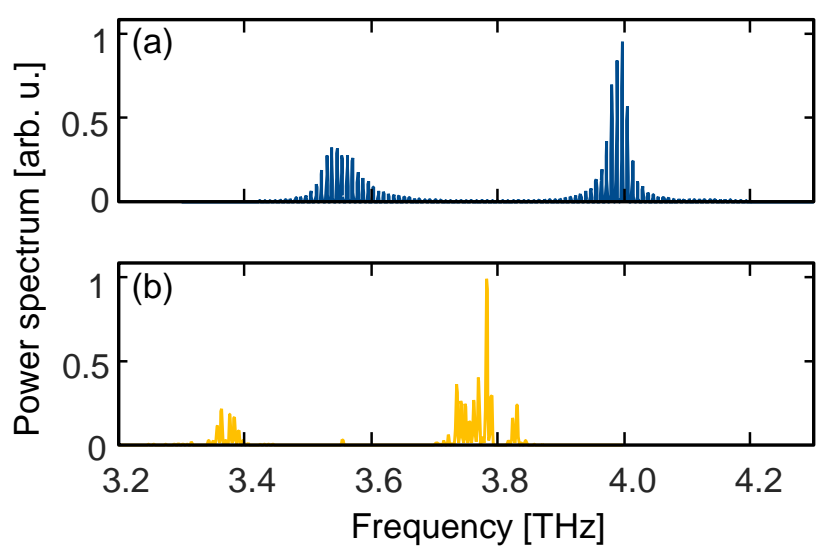

FIG. 20. Optical comb spectrum of a terahertz QCL, as obtained from (a) simulation and (b) experiment.

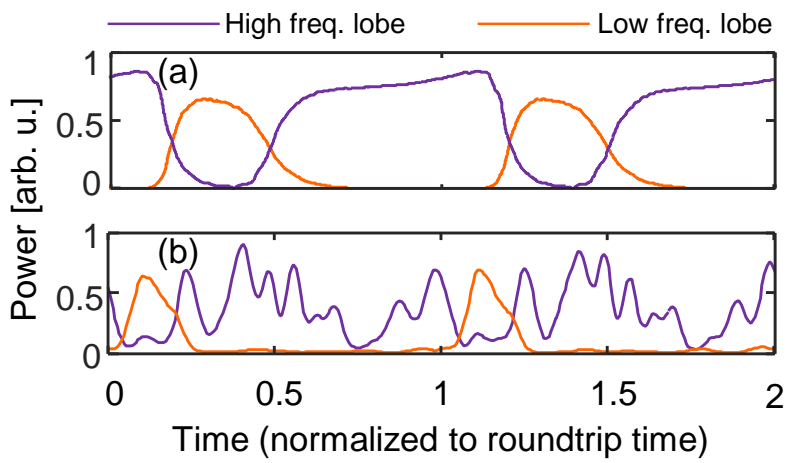

FIG. 21. Instantaneous optical power in the high and low frequency lobe as obtained from (a) simulation and (b) experiment.

been used to model frequency comb operation of QCLs, identifying four-wave mixing as the primary comb forming mechanism and explaining experimentally observed features. 62 |97/322/334/335 In Fig.20, a comparison between simulation and experiment is presented for the power spectrum, $\frac{62}{6}$ generated by a THz QCL for frequency comb generation. 340 Good agreement is found; in particular, a splitting of the comb spectrum into a high and a low frequency lobe is observed in both simulation and experiment. Also the simulated temporal dynamics agrees well with experiment. In Fig. 21, the simulated and measured instantaneous optical power in the high and low frequency lobe of the comb is shown. ${ }^{62}$ Again, good agreement between theory and experiment is obtained, confirming the validity of the MB model. In particular, the temporal switching behavior between the two lobes is reproduced in the simulation. 

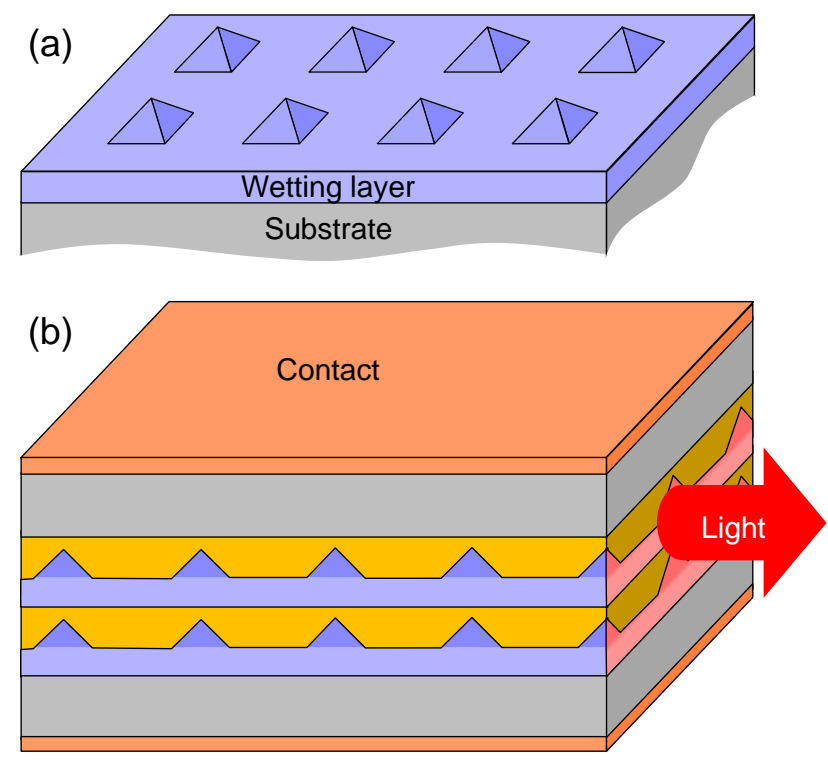

FIG. 22. Schematic illustration of (a) wetting layer with QDs and (b) QD laser.

\section{Quantum Dot Devices}

Due to the strong carrier localization and discrete energy spectrum resulting from the three-dimensional confinement in QDs, they enable lasers and laser amplifiers with excellent gain, threshold, temperature, and dynamic characteristics. 1 . 3 While these devices rely on interband optical transitions, also the possibility has been studied to exploit intraband transitions similarly as in QCLs to obtain lasing in the mid-infrared or terahertz regime. ${ }^{90 \mid 341} 343$ Furthermore, intraband transitions between bound electron or hole states (or from bound to continuum states) have been employed for quantum dot infrared photodetectors 11 13

In contrast to bulk semiconductors and quantum well or wire structures which feature a continuum of states due to the free carrier motion in at least one dimension, the QD possesses a discrete set of energy eigenstates. Thus, the application of phenomenological models based on generic discrete-level MB equations appears to be especially justified for QD systems. Phenomenological two-level $\mathrm{MB}$ equations have been employed for a large range of applications based on QDs. This includes studies of the spatiotemporal dynamics $\$ 344 \sqrt[346]{3}$ and SIT modelocking 121 in QD lasers, FDTD-based MB simulations of QD photonic-crystal-cavity lasers,$\frac{60}{6}$ and QDs coupled to a nanoparticle or cavity $347 \sqrt[349]{34}$ Furthermore, threelevel MB equations have been used, for example to study EIT, ${ }^{248}$ soliton propagation ${ }^{350}$ or all-optical switching 351 in QD structures, and also four-level models have been developed $152 \mid 176$

Optoelectronic applications employing large ensembles of QDs are often fabricated utilizing self-assembly of the QDs on top of an initial quasi-two-dimensional semicon-

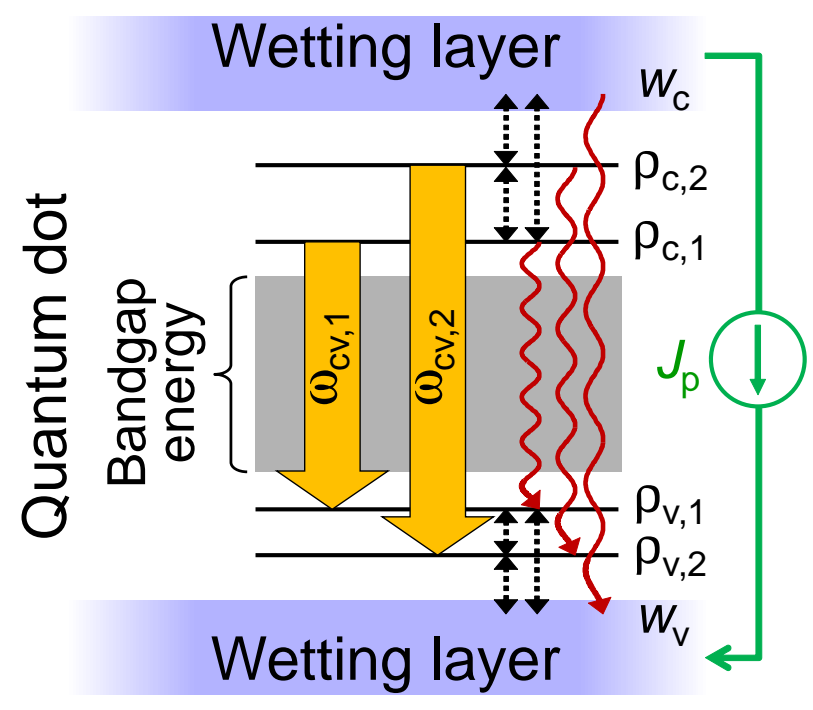

FIG. 23. Schematic energy diagram of QD and wetting layer. The big arrows represent coherent light-matter interaction, the dotted arrows indicate nonradiative intraband transitions, and the wavy arrows represent spontaneous emission.

ductor layer, which is referred to as wetting layer, as sketched in Fig.22(a). The resulting structure is subsequently covered by another layer of suitable semiconductor material. The wetting layer effectively forms a quantum well, which serves as a reservoir for the carriers. The thus obtained QD layer forms the basis of various devices such as QD lasers [see Fig.22(b)], where commonly multiple layers are stacked on top of each other to increase the optical gain.

In Fig.23, a schematic energy diagram of the wetting layer and a QD is shown. A description of the QD dynamics based on the semiconductor Bloch equations, Eqs. (156) and (157), features renormalized transition and Rabi frequencies due to many-body Coulomb interactions. ${ }^{352 / 353}$ These renormalization effects are often neglected so that the conventional Bloch equations, Eq. (17), can be used as a starting point, which are frequently supplemented by a detailed model for Coulomb scattering and other scattering mechanisms in the dissipation term ${ }^{303 / 311}$ In the following, the QD conduction $(\alpha=\mathrm{c})$ and valence $(\alpha=\mathrm{v})$ band states are labeled by an index $i$. Furthermore, as described in Section VIIIB. variations in $\mathrm{QD}$ size result in distributed resonance frequencies, and the associated inhomogeneous broadening is included by dividing the QDs in corresponding subensembles $s$ containing a fraction $p_{s}$ of QDs. The Bloch equations, Eq. (17), thus have to be adapted by replacing the density matrix elements $\rho_{i j}$ with $\rho_{\alpha \alpha^{\prime}, i j}^{s}$ for pairs of states $|\alpha, i\rangle$ and $\left|\alpha^{\prime}, j\right\rangle$, where we write for compactness $\rho_{\alpha \alpha, i j}^{s}=\rho_{\alpha, i j}^{s}, \rho_{\alpha \alpha^{\prime}, i i}^{s}=\rho_{\alpha \alpha^{\prime}, i}^{s}$ and $\rho_{\alpha \alpha, i i}^{s}=\rho_{\alpha, i}^{s}$. For the dipole matrix element vectors $\mathbf{d}_{i j}$, frequencies $\omega_{i j}$ and dephasing rates $\gamma_{i j}$, we proceed analogously. Similarly as for Eq. (156), the $\rho_{\mathrm{v}, i}^{s}$ are taken as the hole oc- 
cupation probability of the $i$ th QD valence band level, i.e., matrix elements $\rho_{i i}$ in Eq. (17) referring to the electron occupation probabilities of QD valence band states have to be substituted by $\left(1-\rho_{\mathrm{v}, i}^{s}\right)$. Apart from the coherent light-matter interaction, incoherent carrier transitions in QD systems mainly occur due to carrier-carrier scattering which gives rise to Auger-type processes, as well as carrier-phonon interactions and spontaneous photon emission. 3031310 Rate equation terms of the form Eq. (9) with phenomenologically chosen parameters are frequently used to model incoherent transitions in $\mathrm{QD}$ systems.60|121|248|344|346|347] For a more detailed modeling, it must be taken into account that important dissipative processes in QDs depend on the occupations of two or more states, and that Pauli blocking is not included in Eq. (9). This can be addressed by using an empirical nonlinear rate equation model $\stackrel{207 / 354}{25}$ or based on a microscopic treatment 30313101311355 For high pump currents, Auger processes, where two carriers scatter from their respective initial to final levels, involving QD and wetting layer states, constitute the dominant scattering process ${ }^{303}$ This includes scattering of two electrons or holes, as well as mixed processes involving an electron transition in the conduction band and a hole transition in the valence band. The associated change of the occupation $\rho_{\alpha, i}^{s}$ is in the following represented by a generic intraband collision term $\left[\partial_{t} \rho_{\alpha, i}^{s}\right]_{\text {intra }},{ }^{303}$ which can be generalized to also include other scattering-induced intraband carrier transitions, e.g., due to electron-phonon interactions. Additionally, spontaneous electron-hole recombination is typically taken into account as an important interband process, which depends on the occupations of the initial and the final state. Within this model, the dissipation terms in Eq. (17b) are substituted by the more general ansatz for incoherent processes ${ }^{303}$

$$
\left[\partial_{t} \rho_{\alpha, i}^{s}\right]_{\mathrm{inc}}=\left[\partial_{t} \rho_{\alpha, i}^{s}\right]_{\mathrm{intra}}-\sum_{j} A_{\mathrm{cv}, i j}^{s} \rho_{\alpha, i}^{s} \rho_{\beta \neq \alpha, j}^{s},
$$

with the spontaneous recombination coefficient $A_{\mathrm{cv}, i j}^{s}$. The Lindblad dephasing rate approach of the form Eq. (12), as also used in Eq. (17a), has been argued to be generally well suited to model dephasing in QDs ${ }^{351}$ The dephasing rates can be calculated based on microscopic models for carrier-carrier and carrierphonon scattering $\sqrt[311355+357]{35}$ or are phenomenologically chosen.248/303/312/351

Considering that $\left[\partial_{t} \rho_{\alpha, i}^{s}\right]_{\text {intra }}$ contains intra-QD transitions as well as carrier exchange between the wetting layers and QD states, this term not only depends on the occupations of the QD states involved, but also on the carrier densities in the wetting layers. Thus, for a closed carrier transport model, the Bloch equations have to be extended by equations for the wetting layers, which can be modeled by

$$
\partial_{t} w_{\alpha}=\frac{J_{\mathrm{p}}}{e}-A_{\mathrm{cv}} w_{\mathrm{c}} w_{\mathrm{v}}-2 n_{2 \mathrm{D}} \sum_{i, s} p_{s}\left[\partial_{t} \rho_{\alpha, i}^{s}\right]_{\mathrm{intra}} .
$$

Here, $w_{\alpha}$ denotes the overall carrier sheet densities in the wetting layers, i.e., $w_{\mathrm{c}}$ is the total number of conduction band electrons in all wetting layers divided by the area $S$ of a wetting layer, and $w_{\mathrm{v}}$ is defined analogously for the valence band holes. $J_{\mathrm{p}}$ denotes the electric pump current density. Furthermore, $n_{2 \mathrm{D}}$ is the overall QD sheet density, and the factor 2 accounts for the spin degeneracy of the QD states. $A_{\mathrm{cv}}$ in Eq. 173 ) is the rate coefficient for spontaneous band-band recombination in the wetting layers. Sometimes the carrier injection from the bulk to the quantum well wetting layers is modeled by additional equations $\sqrt{358}$ For self-assembled quantum dash structures, the wetting layers can be considered in an analogous manner. ${ }^{231359}$

The extended Bloch equations, Eqs. (17), 172) and (173), are then coupled to Maxwell's equations, Eq. 44,, by the polarization current density for inhomogeneously broadened media given in Eq. 154,

$\mathbf{J}_{\mathrm{q}}=\frac{n_{2 \mathrm{D}}}{d_{\mathrm{g}}} \sum_{\alpha, \alpha^{\prime}} \sum_{i, j} \mathbf{d}_{\alpha^{\prime} \alpha, j i} \sum_{s} p_{s}\left(1-2 \delta_{\alpha \mathrm{v}} \delta_{\alpha^{\prime} \mathrm{v}} \delta_{i j}\right) \partial_{t} \rho_{\alpha \alpha^{\prime}, i j}^{s}$,

with the thickness of the gain medium $d_{\mathrm{g}}$. Here, the term $(1-\ldots)$ compensates for the fact that the electron occupation probabilities of $\mathrm{QD}$ valence band states are in our density matrix convention given by $\left(1-\rho_{\mathrm{v}, i}^{s}\right)$. QD lasers and amplifiers usually operate in TE mode, due to the character of the eigenstates for the QD shapes and strains obtained with the widely employed Stranski-Krastanov growth mode 360 A corresponding one-dimensional MB model can be obtained by combining the extended Bloch equations with Maxwell-type equations for TE operation, Eq. (92), where the finite overlap of the QD active region with the mode profile is considered by the field confinement factor, Eq. 88). The contribution of the spontaneous emission processes in Eq. 172 to the optical field is in most cases neglected, but can be considered as discussed in Section VIIIC,

For interband QD devices, optical intersubband transitions can be neglected. The QD interband dipole matrix elements are given by Eq. 222. As discussed in Section IIIC2 2, for the uppermost valence and lowest conduction band states, in good approximation only optical interband transitions between states with equal quantum numbers are allowed, and the corresponding envelope wavefunction overlap in Eq. 222 is $\left\langle\varphi_{i} \mid \varphi_{j}\right\rangle \approx 1$ 146/147 $\mathrm{In}$ fact, for these states close to the band edge the index $i$ is typically associated with a single quantum number, $\stackrel{303}{3}$ due to the typically small aspect ratio of QDs. Under above assumptions, the Bloch equations simplify to

$$
\begin{aligned}
\partial_{t} \rho_{\mathrm{cv}, i}^{s}= & -\mathrm{i} \omega_{\mathrm{cv}, i}^{s} \rho_{\mathrm{cv}, i}^{s}+\mathrm{i} \hbar^{-1} \mathbf{d}_{\mathrm{cv}}\left(1-\rho_{\mathrm{v}, i}^{s}-\rho_{\mathrm{c}, i}^{s}\right) \mathbf{E} \\
& -\gamma_{\mathrm{cv}, i} \rho_{\mathrm{cv}, i}^{s}, \\
\partial_{t} \rho_{\alpha, i}^{s}= & 2 \hbar^{-1} \Im\left\{\mathbf{d}_{\mathrm{vc}} \rho_{\mathrm{cv}, i}^{s}\right\} \mathbf{E}+\left[\partial_{t} \rho_{\alpha, i}^{s}\right]_{\mathrm{intra}} \\
& -A_{\mathrm{cv}, i}^{s} \rho_{\mathrm{c}, i}^{s} \rho_{\mathrm{v}, i}^{s},
\end{aligned}
$$

where $\alpha=\mathrm{c}, \mathrm{v}$, and $\mathbf{d}_{\mathrm{cv}, i}^{s}$ has been approximated by an $s$ and $i$ independent value $\mathbf{d}_{\mathrm{cv}}$. For a closed description of 
the carrier dynamics, Eq. 175 is again supplemented by Eq. 173) ${ }^{303}$ The radiative and nonradiative transitions taken into account in the resulting model are illustrated in Fig.23 The RWA can be applied in the usual manner, as described in Section IIIE. The MB model has been demonstrated to yield good agreement with experimental results for QD lasers and amplifiers, and to be instrumental in interpreting the experimental findings. For example, the ultrafast gain dynamics in a QD amplifier as well as the spatiotemporal dynamics and emission characteristics of a QD laser were experimentally and theoretically studied $\stackrel{361}{36 u r t h e r m o r e, ~ b a s e d ~ o n ~ M B ~}$ simulations of ultrashort laser pulse propagation in a QD amplifier, it could be confirmed that the experimentally observed reshaping was in part due to coherent lightmatter interaction. .2122

As discussed in Section V C2, longitudinal spatial hole burning, i.e., the formation of an inversion grating due to the standing wave pattern in a Fabry-Pérot resonator, is automatically included in full-wave $\mathrm{MB}$ simulations. Assuming that tunneling between adjacent QDs can be neglected, the degradation of the inversion grating is governed by carrier diffusion in the wetting layers, which can be modeled by adding to Eq. (173) a diffusion term of the form Eq. 120 .206|207

\section{CONCLUSION AND OUTLOOK}

The goal of this review has been to discuss in detail the underlying theoretical framework of the MB model, its extension and adaption to certain application areas and types of nanostructures, as well as special analytical solutions and suitable numerical methods. Apart from the intuitive appeal of the model and its adaptability, the relative compactness of the Bloch equations make them highly suitable as an efficient quantum model for the material polarization in computational electrodynamics. As shown in Section VII, their representation as a system of ordinary differential equations in time, where the position coordinates only enter as parameters, allows an efficient coupling to numerical schemes for Maxwell's or related propagation equations, such as the finite-difference time-domain method. This compact form of the Bloch equations is enabled by a mostly phenomenological treatment of dissipation based on the Lindblad formalism and restriction to classical optical fields as well as discrete energy levels. Fully microscopic descriptions of light-matter interaction in a semiconductor, such as the semiconductor MB equations shortly discussed in Section IX A illustrate the limitations of semi-phenomenological Bloch equations, and can serve as a starting point to develop improved compact Bloch equations. As an example, this strategy has been used to model the carrier dynamics in a semiconductor structure with a quasi-continuum of energy levels in the conduction and valence band by macro-

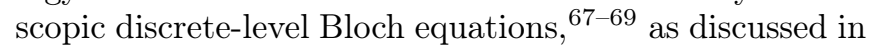
Section IXA
The main requirement for computational models is generally to combine numerical efficiency with accuracy, predictability and versatility. In this context, detailed microscopic theories can quickly become very computationally demanding, which renders them impractical for applications such as device design ${ }^{362}$ Thus, a major goal is to further improve the quantitative accuracy and adaptability of the macroscopic MB equations by extending the model accordingly, however without substantially increasing its numerical complexity. This implies that its general form as a system of a few ordinary differential equations should not be compromised.

Probably the main limitation of the Bloch equations is the phenomenological implementation of dissipation based on the Lindblad formalism. As shortly discussed in Section IX C, an empirical treatment of certain processes, such as Pauli blocking or carrier-carrier scattering, requires a generalization to nonlinear models. Here, special care must be taken to preserve the properties of the density matrix guaranteeing its physical character, which has for example been achieved by suitably extending the Lindblad formalism. ${ }^{73}$

As mentioned in Section IIC the Lindblad model is only realistic from a microscopic point of view if the memory decay of the environment occurs on a faster timescale than the coherent system dynamics and relaxation processes ${ }^{75 / 81}$ Although the macroscopic MB equations often work surprisingly well on the verge of, or even outside, this microscopic validity range, advanced quantitative modeling requires going beyond the Markovian approximation in such cases. An ad hoc extension of the Lindblad approach is obtained by replacing $\mathcal{D}_{k}(\hat{\rho})(t)$ in Eq. (3) with $\int_{0}^{t} K_{k}\left(t-t^{\prime}\right) \mathcal{D}_{k}(\hat{\rho})\left(t^{\prime}\right) \mathrm{d} t^{\prime}$, where $K_{k}$ is the memory kernel ${ }^{363}$ In certain cases, it is sufficient to treat the populations in the usual manner and include memory effects only for dephasing, which requires substituting the dephasing terms $\left[\mathrm{d}_{t} \rho_{\alpha \beta}\right]_{\text {relax }}=-\gamma_{\alpha \beta} \rho_{\alpha \beta}$ in Eq. (12) with $-\gamma_{\alpha \beta} \int_{0}^{t} K_{\alpha \beta}\left(t-t^{\prime}\right) \rho_{\alpha \beta}\left(t^{\prime}\right) \mathrm{d} t^{\prime}$. 364 The characteristic memory time and functional dependence of the memory kernel, such as Gaussian or exponential, depend on the underlying scattering mechanism.338 365 Since the evaluation of convolution integrals is numerically expensive, a representation based on supplemental differential equations is preferential. For exponential memory kernels $K_{\alpha \beta}=\tau_{\alpha \beta}^{-1} \exp \left(-t / \tau_{\alpha \beta}\right)$, such a differential equation is easily derived, with $\mathrm{d}_{t} s=-\tau_{\alpha \beta}^{-1}\left(\gamma_{\alpha \beta} \rho_{\alpha \beta}+s\right)$ where we have introduced $s=\left[\mathrm{d}_{t} \rho_{\alpha \beta}\right]_{\text {relax }}$ for compactness. However, since such modifications obviously do not preserve the Lindblad form of the dissipation terms, a physical behavior of the density matrix is not guaranteed, and in fact highly nonphysical behavior can emerge ${ }^{363}$

It should be pointed out that master equation models with memory effects do not necessarily require con-

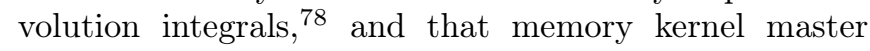
equations can even usually be cast into a time-local form. ${ }^{79|83| 366}$ Thus, a promising approach towards a more generalized treatment of dissipation is to start with the 
Lindblad equation in the form Eq. (4), and to generalize the matrix $\mathcal{D}_{i j m n}$ given in Eq. (6) for an arbitrary set of Lindblad operators. As already mentioned in Section II time dependent Lindblad operators $\hat{L}_{k}(t)$, corresponding to time-varying dissipation rates in Eqs. (8) and (11), are unproblematic. ${ }^{78 / 79}$ Any further generalization of $\mathcal{D}_{i j m n}$ comes at the price of potentially unphysical results. One example is the occurrence of temporarily negative rates in Eqs. (8) or (11), which indeed introduces

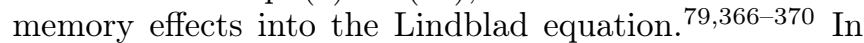
the construction of such a model, care should be taken to avoid unphysical behavior, for example by adding certain constraints. ${ }^{[7 \mid 366]}$ Furthermore, a widely used model of the form Eq. (4) is the Redfield equation, which is derived from microscopic considerations, i.e., a perturbative treatment of a quantum system weakly coupled to the environment $\frac{371372}{1 n}$ this case $\mathcal{D}_{i j m n}$ corresponds to the generally time dependent ${ }^{373}$ Redfield tensor, which is directly related to the system-environment coupling and environment Hamiltonians. $\frac{374}{3}$ The main advantages of the Redfield model are its strong connection to microscopic physics, and to some extent the inclusion of shortterm memory effects. $\frac{373 \mid 375}{}$ However, in its commonly used form, the Redfield equation does not guarantee positivity of the density matrix which can lead to negative state occupations. In practice, the emergence of this unphysical behavior appears to be a minor problem, 376,378 and can also be cured. $373 \mid 379$

Finally, applying the Lindblad formalism to a suitably extended state space, a non-Markovian evolution with arbitrarily long memory times and strong initial correla-

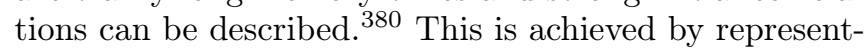
ing the reduced system density matrix $\rho_{\mathrm{s}}$ with dimension $N$ as a sum of a certain number $M$ of positive matrices $\rho_{i}$, i.e., $\rho_{\mathrm{s}}=\sum_{i} \rho_{i}$ where the traces of the $\rho_{i}$ must add up to one. Then, a big block diagonal density matrix $\rho$ with dimension $M N$ is constructed from the $\rho_{i}$, and the evolution of $\rho$ is modeled by a Lindblad equation for the extended system, where the operators are required to preserve the block diagonal form of $\rho$. This leads to $M$ coupled evolution equations for the $\rho_{i}$, where the dynamics is now defined by $M$ arbitrary Hermitian operators $\hat{H}_{i}$ and $M^{2}$ sets of arbitrary dissipation operators $\left\{\hat{L}_{1}^{i j}, \ldots, \hat{L}_{K}^{i j}\right\}$. In this way, although the dynamics of $\rho$ is Markovian, the model can describe a highly nonMarkovian evolution of $\rho_{\mathrm{s}}$, while intrinsically preserving the physical properties of $\rho_{\mathrm{s}}$. An interesting subcase is when the evolution equations of the $\rho_{i}$ are decoupled, i.e., $\hat{L}_{k}^{i j}=\hat{L}_{k}^{i j} \delta_{i j}{ }^{[380 \mid 381}$ In this case, the evolution of each $\rho_{i}$ is described by an equation of the Lindblad form Eq. (3), but still a non-Markovian dynamics of $\rho_{\mathrm{s}}$ is obtained. Of course, for $M=1$, the standard Markovian Lindblad dynamics is recovered.

\section{CONFLICTS OF INTEREST}

The authors declare no conflicts of interest.

\section{SUPPORTING INFORMATION}

Supporting information is available from the Wiley Online Library or from the author.

\section{ACKNOWLEDGMENTS}

We thank Gabriela Slavcheva for valuable comments and suggestions. The authors acknowledge financial support by the European Union's Horizon 2020 research and innovation programme under grant agreement No 820419 - Qombs Project "Quantum simulation and entanglement engineering in quantum cascade laser frequency combs" (FET Flagship on Quantum Technologies), and by the German Research Foundation (DFG) within the Heisenberg program (JI 115/4-2).

${ }^{1}$ N. Kirstaedter, N. Ledentsov, M. Grundmann, D. Bimberg, V. Ustinov, S. Ruvimov, M. Maximov, P. S. Kop'ev, Z. I. Alferov, U. Richter, P. Werner, U. Gosele, J. Heydenreich, Electron. Lett. 1994, 30, 1416.

${ }^{2}$ N. Ledentsov, V. Ustinov, V. Shchukin, P. Kop'ev, Z. I. Alferov, D. Bimberg, Semiconductors 1998, 32, 343.

${ }^{3}$ D. Huffaker, G. Park, Z. Zou, O. Shchekin, D. Deppe, Appl. Phys. Lett. 1998, 73, 2564.

${ }^{4}$ J. P. Reithmaier, G. Eisenstein, A. Forchel, Proc. IEEE 2007, 95, 1779.

${ }^{5}$ X. Duan, Y. Huang, R. Agarwal, C. M. Lieber, Nature 2003, 421, 241.

${ }^{6}$ B. Mayer, A. Regler, S. Sterzl, T. Stettner, G. Koblmüller, M. Kaniber, B. Lingnau, K. Lüdge, J. Finley, Nat. Commun. 2017, 8, 15521.

${ }^{7}$ J. Faist, F. Capasso, D. L. Sivco, C. Sirtori, A. L. Hutchinson, A. Y. Cho, Science 1994, 264, 553.

${ }^{8}$ D. Hofstetter, M. Beck, J. Faist, Appl. Phys. Lett. 2002, 81, 2683.

${ }^{9}$ L. Gendron, M. Carras, A. Huynh, V. Ortiz, C. Koeniguer, V. Berger, Appl. Phys. Lett. 2004, 85, 2824.

${ }^{10}$ B. Levine, J. Appl. Phys. 1993, 74, R1.

${ }^{11}$ J. Phillips, K. Kamath, P. Bhattacharya, Appl. Phys. Lett. 1998, 72, 2020.

${ }^{12}$ S.-W. Lee, K. Hirakawa, Y. Shimada, Appl. Phys. Lett. 1999, $75,1428$.

${ }^{13}$ H. Liu, M. Gao, J. McCaffrey, Z. Wasilewski, S. Fafard, Appl. Phys. Lett. 2001, 78, 79.

${ }^{14}$ I. I. Rabi, Phys. Rev. 1937, 51, 652.

${ }^{15}$ A. Zrenner, E. Beham, S. Stufler, F. Findeis, M. Bichler, G. Abstreiter, Nature 2002, 418, 612.

${ }^{16}$ S. Cundiff, A. Knorr, J. Feldmann, S. Koch, E. Göbel, H. Nickel, Phys. Rev. Lett. 1994, 73, 1178.

${ }^{17}$ A. Schülzgen, R. Binder, M. Donovan, M. Lindberg, K. Wundke, H. Gibbs, G. Khitrova, N. Peyghambarian, Phys. Rev. Lett. 1999, 82, 2346.

${ }^{18}$ H. Choi, V.-M. Gkortsas, L. Diehl, D. Bour, S. Corzine, J. Zhu, G. Höfler, F. Capasso, F. X. Kärtner, T. B. Norris, Nature Photon. 2010, 4, 706 .

${ }^{19}$ T. Stievater, X. Li, D. G. Steel, D. Gammon, D. Katzer, D. Park, C. Piermarocchi, L. Sham, Phys. Rev. Lett. 2001, 87, 133603.

${ }^{20}$ H. Kamada, H. Gotoh, J. Temmyo, T. Takagahara, H. Ando, Phys. Rev. Lett. 2001, 87, 246401.

${ }^{21}$ M. Kolarczik, N. Owschimikow, J. Korn, B. Lingnau, Y. Kaptan, D. Bimberg, E. Schöll, K. Lüdge, U. Woggon, Nat. Commun. 2013, 4, 2953.

${ }^{22}$ O. Karni, A. Capua, G. Eisenstein, V. Sichkovskyi, V. Ivanov, J. P. Reithmaier, Opt. Express 2013, 21, 26786. 
${ }^{23}$ A. Capua, O. Karni, G. Eisenstein, J. P. Reithmaier, Phys. Rev. B 2014, 90, 045305.

${ }^{24}$ S. L. McCall, E. L. Hahn, Phys. Rev. Lett. 1967, 18, 908.

${ }^{25}$ S. L. McCall, E. L. Hahn, Phys. Rev. 1969, 183, 457.

${ }^{26}$ S. Schneider, P. Borri, W. Langbein, U. Woggon, J. Förstner, A. Knorr, R. Sellin, D. Ouyang, D. Bimberg, Appl. Phys. Lett. 2003, 83, 3668 .

${ }^{27}$ V. V. Kozlov, Phys. Rev. A 1997, 56, 1607.

${ }^{28}$ V. Kalosha, M. Müller, J. Herrmann, J. Opt. Soc. Am. B 1999, $16,323$.

${ }^{29}$ V. V. Kozlov, N. N. Rosanov, S. Wabnitz, Phys. Rev. A 2011 84, 053810

${ }^{30}$ C. R. Menyuk, M. A. Talukder, Phys. Rev. Lett. 2009, 102, 023903.

${ }^{31}$ R. Arkhipov, M. Arkhipov, I. Babushkin, Opt. Commun. 2016 , 361, 73.

${ }^{32}$ L. V. Hau, S. E. Harris, Z. Dutton, C. H. Behroozi, Nature 1999, 397, 594.

${ }^{33}$ C. Liu, Z. Dutton, C. H. Behroozi, L. V. Hau, Nature 2001, 409, 490.

${ }^{34}$ D. Phillips, A. Fleischhauer, A. Mair, R. Walsworth, M. D. Lukin, Phys. Rev. Lett. 2001, 86, 783.

${ }^{35}$ J. B. Khurgin, J. Opt. Soc. Am. B 2005, 22, 1062.

${ }^{36}$ R. M. Camacho, C. J. Broadbent, I. Ali-Khan, J. C. Howell, Phys. Rev. Lett. 2007, 98, 043902.

${ }^{37}$ O. Firstenberg, M. Shuker, N. Davidson, A. Ron, Phys. Rev. Lett. 2009, 102, 043601.

${ }^{38}$ M. Lukin, S. Yelin, M. Fleischhauer, Phys. Rev. Lett. 2000, 84, 4232.

${ }^{39}$ A. Turukhin, V. Sudarshanam, M. Shahriar, J. Musser, B. Ham, P. Hemmer, Phys. Rev. Lett. 2001, 88, 023602.

${ }^{40}$ M. S. Bigelow, N. N. Lepeshkin, R. W. Boyd, Phys. Rev. Lett. 2003, 90, 113903.

${ }^{41}$ P. Ginzburg, M. Orenstein, Opt. Express 2006, 14, 12467.

${ }^{42}$ H. Borges, L. Sanz, J. Villas-Bôas, O. D. Neto, A. Alcalde, Phys. Rev. B 2012, 85, 115425.

${ }^{43}$ P. Tzenov, C. Jirauschek in Proc. SPIE 10226, 19th International Conference and School on Quantum Electronics: Laser Physics and Applications, International Society for Optics and Photonics, p. 1022603.

${ }^{44}$ J.-H. Wu, J.-Y. Gao, J.-H. Xu, L. Silvestri, M. Artoni, G. La Rocca, F. Bassani, Phys. Rev. Lett. 2005, 95, 057401.

${ }^{45}$ J. Gea-Banacloche, M. Mumba, M. Xiao, Phys. Rev. B 2006, 74, 165330

${ }^{46}$ F. Bloch, Phys. Rev. 1946, 70, 460.

${ }^{47}$ R. P. Feynman, F. L. Vernon Jr, R. W. Hellwarth, J. Appl. Phys. 1957, 28, 49.

${ }^{48}$ I. Abella, N. Kurnit, S. Hartmann, Phys. Rev. 1966, 141, 391.

${ }^{49}$ L. Allen, J. H. Eberly, Optical Resonance and Two-Level Atoms, Vol. 28, Courier Corporation, 1987.

${ }^{50}$ G. Lindblad, Commun. Math. Phys. 1976, 48, 119.

${ }^{51}$ V. Gorini, A. Kossakowski, E. C. G. Sudarshan, J. Math. Phys. 1976, 17, 821.

${ }^{52}$ A. Taflove, S. C. Hagness, Computational Electrodynamics: The Finite-Difference Time-Domain Method, Artech House, 2005.

${ }^{53}$ G. Slavcheva, J. M. Arnold, I. Wallace, R. W. Ziolkowski, Phys. Rev. A 2002, 66, 063418.

${ }^{54}$ A. Klaedtke, O. Hess, Opt. Express 2006, 14, 2744.

${ }^{55}$ M. Sukharev, A. Nitzan, Phys. Rev. A 2011, 84, 043802.

${ }^{56}$ A. Pusch, S. Wuestner, J. M. Hamm, K. L. Tsakmakidis, O. Hess, ACS Nano 2012, 6, 2420

${ }^{57}$ K. Lopata, D. Neuhauser, J. Chem. Phys. 2009, 131, 014701.

${ }^{58}$ H. Takeda, S. John, Phys. Rev. A 2011, 83, 053811.

${ }^{59}$ M. Dridi, G. C. Schatz, J. Opt. Soc. Am. B 2013, 30, 2791.

${ }^{60}$ W. Cartar, J. Mørk, S. Hughes, Phys. Rev. A 2017, 96, 023859.

${ }^{61} \mathrm{M}$. Riesch, P. Tzenov, C. Jirauschek in 2018 2nd URSI Atlantic Radio Science Meeting (AT-RASC), IEEE, pp. 1-4.

${ }^{62} \mathrm{P}$. Tzenov, D. Burghoff, Q. Hu, C. Jirauschek, Opt. Express 2016, 24, 23232

${ }^{63}$ C. M. Bowden, J. P. Dowling, Phys. Rev. A 1993, 47, 1247.
${ }^{64}$ G. Y. Slepyan, S. Maksimenko, A. Hoffmann, D. Bimberg, Phys. Rev. A 2002, 66, 063804.

${ }^{65}$ W. W. Chow, S. W. Koch, M. I. Sargent, Semiconductor-Laser Physics, Springer Science \& Business Media, 2012.

${ }^{66}$ H. Haug, S. W. Koch, Quantum Theory of the Optical and Electronic Properties of Semiconductors, World Scientific Publishing Company, 2009.

${ }^{67}$ C. Ning, R. Indik, J. Moloney, IEEE J. Quantum Electron. 1997, 33, 1543

${ }^{68}$ J. Yao, G. P. Agrawal, P. Gallion, C. M. Bowden, Opt. Commun. 1995, 119, 246.

${ }^{69}$ S. Balle, Opt. Commun. 1995, 119, 227.

${ }^{70}$ R. Rosati, F. Rossi, Phys. Rev. B 2014, 89, 205415.

${ }^{71}$ D. Stepanenko, G. Burkard, G. Giedke, A. Imamoglu, Phy. Rev. Lett. 2006, 96, 136401.

${ }^{72}$ S. G. Schirmer, A. I. Solomon, Phys. Rev. A 2004, 70, 022107.

${ }^{73}$ R. Rosati, R. C. Iotti, F. Dolcini, F. Rossi, Phys. Rev. B 2014, 90, 125140.

${ }^{74}$ A. Steinhoff, P. Gartner, M. Florian, F. Jahnke, Phys. Rev. B 2012, 85, 205144.

${ }^{75}$ H.-P. Breuer, F. Petruccione, The Theory of Open Quantum Systems, Oxford University Press, 2002.

${ }^{76}$ J. Preskill, Lecture Notes for Physics 229: Quantum Information and Computation, California Institute of Technology, 1998.

${ }^{77}$ K. Kraus, Ann. Phys. (N. Y.) 1971, 64, 311.

${ }^{78}$ H.-P. Breuer, Phys. Rev. A 2004, 70, 012106.

${ }^{79}$ C. M. Kropf, C. Gneiting, A. Buchleitner, Phys. Rev. X 2016 , $6,031023$.

${ }^{80}$ E. B. Davies, Commun. Math. Phys. 1974, 39, 91.

${ }^{81}$ M. Le Bellac, Quantum Physics, Cambridge University Press, 2011.

${ }^{82}$ M. Kantner, U. Bandelow, T. Koprucki, H.-J. Wünsche in $\mathrm{Nu}$ merical Simulation of Optoelectronic Devices (NUSOD), 2015 International Conference on, IEEE, pp. 151-152.

${ }^{83}$ D. Chruściński, A. Kossakowski, Phys. Rev. Lett. 2010, 104, 070406.

${ }^{84}$ M. Grifoni, P. Hänggi, Phys. Rep. 1998, 304, 229.

${ }^{85}$ D. K. Oi, S. G. Schirmer, Phys. Rev. A 2012, 86, 012121.

${ }^{86}$ P. Rebentrost, M. Mohseni, I. Kassal, S. Lloyd, A. AspuruGuzik, New J. Phys. 2009, 11, 033003.

${ }^{87}$ S. Fathololoumi, E. Dupont, C. Chan, Z. Wasilewski, S. Laframboise, D. Ban, A. Mátyás, C. Jirauschek, Q. Hu, H. Liu, Opt. Express 2012, 20, 3866.

${ }^{88}$ T. Dinh, A. Valavanis, L. Lever, Z. Ikonić, R. Kelsall, Phys. Rev. B 2012, 85, 235427.

${ }^{89}$ E. Dupont, S. Fathololoumi, H. C. Liu, Phys. Rev. B 2010, 81, 205311.

${ }^{90}$ B. A. Burnett, B. S. Williams, Phys. Rev. B 2014, 90, 155309.

${ }^{91}$ H. Callebaut, Q. Hu, J. Appl. Phys. 2005, 98, 104505.

${ }^{92}$ G. Pfanner, M. Seliger, U. Hohenester, Phys. Rev. B 2008, 78, 195410.

${ }^{93}$ B. Palmieri, D. Abramavicius, S. Mukamel, J. Chem. Phys. 2009, 130, 204512.

${ }^{94}$ G. Kiršanskas, M. Franckié, A. Wacker, Phys. Rev. B 2018, 97 , 035432.

${ }^{95}$ S. Kumar, Q. Hu, Phys. Rev. B 2009, 80, 245316.

${ }^{96}$ R. Terazzi, J. Faist, New J. Phys. 2010, 12, 033045.

${ }^{97} \mathrm{P}$. Tzenov, D. Burghoff, Q. Hu, C. Jirauschek, IEEE Trans. Terahertz Sci. Technol. 2017, 7, 351.

${ }^{98}$ C. Jirauschek, J. Appl. Phys. 2017, 122, 133105.

${ }^{99}$ A. Gordon, D. Majer, Phys. Rev. B 2009, 80, 195317.

${ }^{100}$ Y. Dubi, M. Di Ventra, Nano Lett. 2008, 9, 97.

${ }^{101}$ W. Freeman, Phys. Rev. B 2016, 93, 205301.

${ }^{102}$ R. W. Ziolkowski, J. M. Arnold, D. M. Gogny, Phys. Rev. A 1995, 52, 3082.

${ }^{103}$ S. Hughes, Phys. Rev. Lett. 1998, 81, 3363.

${ }^{104}$ V. Kalosha, J. Herrmann, Phys. Rev. Lett. 1999, 83, 544.

${ }^{105}$ O. Mücke, T. Tritschler, M. Wegener, U. Morgner, F. Kärtner, Phys. Rev. Lett. 2001, 87, 057401. 
${ }^{106}$ J. R. Freeman, J. Maysonnave, S. Khanna, E. H. Linfield, A. G. Davies, S. S. Dhillon, J. Tignon, Phys. Rev. a 2013, 87, 063817.

${ }^{107}$ S. Mukamel, Annu. Rev. Phys. Chem. 1990, 41, 647.

${ }^{108}$ N. Gisin, I. C. Percival, Journal of Physics A: Mathematical and General 1992, 25, 5677.

${ }^{109}$ P. Meystre, M. Sargent, Elements of Quantum Optics, Springer Science \& Business Media, 2013.

${ }^{110}$ M. O. Scully, M. S. Zubairy, Quantum Optics, Cambridge University Press, 1997.

${ }^{111}$ M. W. Walser, C. H. Keitel, A. Scrinzi, T. Brabec, Phys. Rev. Lett. 2000, 85, 5082 .

${ }^{112}$ S. Stobbe, P. T. Kristensen, J. E. Mortensen, J. M. Hvam, J. Mørk, P. Lodahl, Phys. Rev. B 2012, 86, 085304.

${ }^{113}$ P. Lodahl, S. Mahmoodian, S. Stobbe, Rev. Mod. Phys. 2015, $87,347$.

${ }^{114}$ K. Rzązewski, R. W. Boyd, J. Mod. Opt. 2004, 51, 1137.

${ }^{115}$ D. Bauer, D. Milošević, W. Becker, Phys. Rev. A 2005, 72, 023415 .

${ }^{116}$ T. Yoshie, A. Scherer, J. Hendrickson, G. Khitrova, H. Gibbs, G. Rupper, C. Ell, O. Shchekin, D. Deppe, Nature 2004, 432, 200.

${ }^{117}$ D. Englund, A. Majumdar, A. Faraon, M. Toishi, N. Stoltz, P. Petroff, J. Vučković, Phys. Rev. Lett. 2010, 104, 073904.

${ }^{118}$ O. Hess, T. Kuhn, Phys. Rev. A 1996, 54, 3347.

${ }^{119}$ C. Jirauschek, T. Kubis, Appl. Phys. Rev. 2014, 1, 011307.

${ }^{120}$ M. A. Talukder, C. R. Menyuk, Opt. Express 2014, 22, 15608.

${ }^{121}$ R. Arkhipov, M. Arkhipov, I. Babushkin, N. Rosanov, Opt. Lett. 2016, 41, 737 .

${ }^{122} \mathrm{P}$. Tzenov, I. Babushkin, R. Arkhipov, M. Arkhipov, N. Rosanov, U. Morgner, C. Jirauschek, New J. Phys. 2018, 20, 053055 .

${ }^{123}$ A.-B. Chen, A. Sher, Phys. Rev. B 1980, 22, 3886.

${ }^{124}$ G. Bastard, Wave Mechanics Applied to Semiconductor Heterostructures, Les Editons de Physique, Paris, 1988.

${ }^{125}$ S. Datta, Quantum Transport: Atom to Transistor, Cambridge University Press, 2005.

${ }^{126}$ C. Jirauschek, IEEE J. Quantum Electron. 2009, 45, 1059.

${ }^{127}$ U. Ekenberg, Phys. Rev. B 1989, 40, 7714.

${ }^{128}$ D. F. Nelson, R. C. Miller, D. A. Kleinman, Phys. Rev. B 1987, 35, 7770 .

${ }^{129}$ E. O. Kane, J. Phys. Chem. Solids 1957, 1, 249.

${ }^{130}$ E. O. Kane, Handbook on Semiconductors 1982, 1, 193.

${ }^{131}$ J. M. Luttinger, W. Kohn, Phys. Rev. 1955, 97, 869.

${ }^{132}$ G. L. Bir, G. E. Pikus, Symmetry and Strain-Induced Effects in Semiconductors, Wiley New York, 1974.

${ }^{133}$ C. R. Pidgeon, R. Brown, Phys. Rev. 1966, 146, 575.

${ }^{134}$ O. Stier, M. Grundmann, D. Bimberg, Phys. Rev. B 1999, 59, 5688.

${ }^{135}$ G. Baraff, D. Gershoni, Phys. Rev. B 1991, 43, 4011.

${ }^{136}$ D. J. Paul, Phys. Rev. B 2008, 77, 155323.

${ }^{137}$ B. A. Foreman, Phys. Rev. B 1993, 48, 4964.

${ }^{138}$ U. Rössler, Solid State Commun. 1984, 49, 943.

${ }^{139}$ S. Ridene, K. Boujdaria, H. Bouchriha, G. Fishman, Phys. Rev. B 2001, 64, 085329.

${ }^{140}$ J. H. Davies, The Physics of Low-dimensional Semiconductors, Cambridge University Press, Cambridge, 1997.

${ }^{141}$ M. Burt, J. Phys. Condens. Matter 1993, 5, 4091.

${ }^{142}$ B. D. Gerardot, D. Brunner, P. A. Dalgarno, P. Öhberg, S. Seidl, M. Kroner, K. Karrai, N. G. Stoltz, P. M. Petroff, R. J. Warburton, Nature 2008, 451, 441.

${ }^{143}$ K. Karlsson, V. Troncale, D. Oberli, A. Malko, E. Pelucchi, A. Rudra, E. Kapon, Appl. Phys. Lett. 2006, 89, 251113.

${ }^{144}$ Y.-M. Niquet, D. C. Mojica, Phys. Rev. B 2008, 77, 115316.

${ }^{145}$ S. Cortez, O. Krebs, P. Voisin, J. Gérard, Phys. Rev. B 2001, 63, 233306

${ }^{146}$ D. Bimberg, M. Grundmann, N. N. Ledentsov, Quantum Dot Heterostructures, John Wiley \& Sons, 1999.

${ }^{147}$ D. Bimberg, Semiconductors 1999, 33, 951.

${ }^{148}$ U. Woggon, Optical Properties of Semiconductor Quantum Dots, Springer, 1997.
${ }^{149} \mathrm{~J}$. Finley in Handbook of Self Assembled Semiconductor Nanostructures for Novel Devices in Photonics and Electronics, Elsevier, 2008, pp. 476-504.

${ }^{150}$ F. T. Hioe, J. H. Eberly, Phys. Rev. Lett. 1981, 47, 838.

${ }^{151}$ G. Slavcheva, J. M. Arnold, R. W. Ziolkowski, IEEE J. Sel. Top. Quantum Electron. 2003, 9, 929.

${ }^{152}$ G. Slavcheva, Phys. Rev. B 2008, 77, 115347.

${ }^{153}$ G. Slavcheva, P. Roussignol, New J. Phys. 2010, 12, 103004.

${ }^{154}$ R. Marskar, U. Österberg, Opt. Express 2011, 19, 16784.

${ }^{155}$ F. Bloch, A. Siegert, Phys. Rev. 1940, 57, 522.

${ }^{156}$ N. Bloembergen, Nonlinear Optics, World Scientific, Singapore, 1996.

${ }^{157}$ R. W. Boyd, Nonlinear Optics, Academic, 2003.

${ }^{158}$ A. Wacker, Phys. Rep. 2002, 357, 1.

${ }^{159} \mathrm{M}$. Wegener, Extreme Nonlinear Optics: An Introduction, Springer Science \& Business Media, 2005.

${ }^{160}$ N. Owschimikow, C. Gmachl, A. Belyanin, V. Kocharovsky, D. L. Sivco, R. Colombelli, F. Capasso, A. Y. Cho, Phys. Rev. Lett. 2003, 90, 043902.

${ }^{161}$ D. Englund, A. Faraon, I. Fushman, N. Stoltz, P. Petroff, J. Vučković, Nature 2007, 450, 857.

${ }^{162}$ K. Srinivasan, O. Painter, Nature 2007, 450, 862.

${ }^{163}$ M. Belkin, F. Capasso, F. Xie, A. Belyanin, M. Fischer, A. Wittmann, J. Faist, Appl. Phys. Lett. 2008, 92, 201101.

${ }^{164}$ W. Maineult, L. Ding, P. Gellie, P. Filloux, C. Sirtori, S. Barbieri, T. Akalin, J.-F. Lampin, I. Sagnes, H. E. Beere, D. A. Ritchie, Appl. Phys. Lett. 2010, 96, 021108.

${ }^{165}$ A. Calvar, M. Amanti, M. Renaudat St-Jean, S. Barbieri, A. Bismuto, E. Gini, M. Beck, J. Faist, C. Sirtori, Appl. Phys. Lett. 2013, 102, 181114.

${ }^{166}$ M. R. St-Jean, M. I. Amanti, A. Bernard, A. Calvar, A. Bismuto, E. Gini, M. Beck, J. Faist, H. Liu, C. Sirtori, Laser Photon. Rev. 2014, 8, 443 .

${ }^{167}$ F. Wang, K. Maussang, S. Moumdji, R. Colombelli, J. R. Freeman, I. Kundu, L. Li, E. H. Linfield, A. G. Davies, J. Mangeney, J. Tignon, S. S. Dhillon, Optica 2015, 2, 944.

${ }^{168}$ J. Faist, G. Villares, G. Scalari, M. Rösch, C. Bonzon, A. Hugi, M. Beck, Nanophotonics 2016, 5, 272.

${ }^{169}$ G. Grau, W. Freude, Optische Nachrichtentechnik - Eine Einführung, Springer, Berlin, 1991.

${ }^{170}$ A. Yariv, Quantum Electronics, John Wiley \& Sons, New York, 1989.

${ }^{171}$ M. Sargent III, M. O. Scully, J. W. E. Lamb, Laser Physics, Addison-Wesley, Reading, MA, 1987.

${ }^{172}$ R. G. Brewer, R. Shoemaker, Phys. Rev. A 1972, 6, 2001.

${ }^{173}$ G. M. Slavcheva, J. M. Arnold, R. W. Ziolkowski, IEEE J. Sel. Top. Quantum Electron. 2004, 10, 1052.

${ }^{174}$ P. Siddons, J. Phys. B 2014, 47, 093001.

${ }^{175}$ G. Slavcheva, O. Hess, Phys. Rev. A 2005, 72, 053804.

${ }^{176}$ G. Slavcheva, M. Koleva, A. Rastelli, Phys. Rev. B 2019, 99, 115433.

${ }^{177}$ X. Song, S. Gong, R. Li, Z. Xu, Phys. Rev. A 2006, 74, 015802.

${ }^{178}$ N. Schulz, K. Bierwirth, F. Arndt, U. Koster, IEEE Trans. Microw. Theory Techn. 1990, 38, 722 .

${ }^{179}$ A. S. Sudbo, Pure Appl. Opt. 1994, 3, 381.

${ }^{180} \mathrm{~K}$. Chiang, Opt. Lett. 1991, 16, 714 .

${ }^{181}$ G. Agrawal, Nonlinear Fiber Optics, Academic, New York, 2001.

${ }^{182}$ E. Schrödinger, Ann. Phys. (Berl.) 1926, 385, 437.

${ }^{183}$ M. M. Sternheim, J. F. Walker, Phys. Rev. C 1972, 6, 114.

${ }^{184} \mathrm{~T}$. Visser, H. Blok, B. Demeulenaere, D. Lenstra, IEEE J. Quantum Electron. 1997, 33, 1763.

${ }^{185}$ J. D. Jackson, Classical Electrodynamics, Wiley \& Sons, New York, 1999.

${ }^{186}$ Q. Y. Lu, N. Bandyopadhyay, S. Slivken, Y. Bai, M. Razeghi, Appl. Phys. Lett. 2011, 99, 131106.

${ }^{187}$ M. Born, E. Wolf, Principles of Optics: Electromagnetic Theory of Propagation, Interference and Diffraction of Light, Cambridge University Press, Cambridge, 1999.

${ }^{188}$ R. Bräuer, O. Bryngdahl, Appl. Opt. 1994, 33, 7875. 
${ }^{189}$ A. Maimistov, E. Manykin, Zh. Eksp. Teor. Fiz 1983, 85, 1177.

${ }^{190}$ E. Doktorov, R. Vlasov, Opt. Acta 1983, 30, 223

${ }^{191}$ M. Nakazawa, E. Yamada, H. Kubota, Phys. Rev. Lett. 1991, $66,2625$.

${ }_{192}$ R. Guo, H.-Q. Hao, Ann. Phys. (N. Y.) 2014, 344, 10.

${ }^{193}$ F. DeMartini, C. Townes, T. Gustafson, P. Kelley, Phys. Rev. 1967, 164, 312

${ }^{194}$ F. M. Mitschke, L. F. Mollenauer, Opt. Lett. 1986, 11, 659.

195 J. P. Gordon, Opt. Lett. 1986, 11, 662.

${ }^{196}$ K. Nakkeeran, K. Porsezian, J. Phys. A 1995, 28, 3817.

${ }^{197}$ S. Kohen, B. S. Williams, Q. Hu, J. Appl. Phys. 2005, 97, 053106.

${ }^{198}$ J. Butler, J. Zoroofchi, IEEE J. Quantum Electron. 1974, 10, 809.

${ }^{199}$ T. Ikegami, IEEE J. Quantum Electron. 1972, 8, 470.

${ }^{200}$ P. C. Kendall, D. A. Roberts, P. N. Robson, M. J. Adams, M. J. Robertson, IEEE Photon. Technol. Lett. 1993, 5, 148.

${ }^{201}$ F. K. Reinhart, I. Hayashi, M. B. Panish, J. Appl. Phys. 1971, 42, 4466.

${ }^{202}$ C. Y. Wang, L. Diehl, A. Gordon, C. Jirauschek, F. X. Kärtner, A. Belyanin, D. Bour, S. Corzine, G. Höfler, M. Troccoli, J. Faist, F. Capasso, Phys. Rev. A 2007, 75, 031802.

${ }^{203}$ O. Hess, T. Kuhn, Phys. Rev. A 1996, 54, 3360.

${ }^{204}$ H. C. Torrey, Phys. Rev. 1956, 104, 563.

${ }^{205}$ T. Ando, A. B. Fowler, F. Stern, Rev. Mod. Phys. 1982, 54, 437.

${ }^{206}$ L. V. Asryan, R. A. Suris, IEEE J. Quantum Electron. 2000, $36,1151$.

${ }^{207}$ A. Capua, O. Karni, G. Eisenstein, IEEE J. Sel. Top. Quantum Electron. 2013, 19, 1900410.

${ }^{208}$ A. Gordon, C. Y. Wang, L. Diehl, F. Kärtner, A. Belyanin, D. Bour, S. Corzine, G. Höfler, H. Liu, H. Schneider, T. Maier, M. Troccoli, J. Faist, F. Capasso, Phys. Rev. A 2008, 77, 053804 .

${ }^{209}$ N. Vukovic, J. Radovanovic, V. Milanovic, D. Boiko, Opt. Quant. Electron. 2016, 48, 254.

${ }^{210}$ H. Torrey, Phys. Rev. 1949, 76, 1059.

${ }^{211}$ A. Muller, E. B. Flagg, P. Bianucci, X. Wang, D. G. Deppe, W. Ma, J. Zhang, G. Salamo, M. Xiao, C.-K. Shih, Phys. Rev. Lett. 2007, 99, 187402 .

${ }^{212}$ X. Xu, B. Sun, P. R. Berman, D. G. Steel, A. S. Bracker, D. Gammon, L. J. Sham, Science 2007, 317, 929.

${ }^{213}$ M. Wagner, H. Schneider, D. Stehr, S. Winnerl, A. M. Andrews, S. Schartner, G. Strasser, M. Helm, Phys. Rev. Lett. 2010, 105, 167401.

${ }^{214}$ C. Henry, IEEE J. Quantum Electron. 1982, 18, 259.

${ }^{215}$ D. Bimberg, N. Kirstaedter, N. Ledentsov, Z. I. Alferov, P. Kop'ev, V. Ustinov, IEEE J. Sel. Top. Quantum Electron. 1997, 3, 196.

${ }^{216}$ C. Jirauschek, Opt. Express 2010, 18, 25922.

${ }^{217}$ K.-J. Boller, A. Imamoğlu, S. E. Harris, Phys. Rev. Lett. 1991, 66, 2593.

${ }^{218}$ M. Fleischhauer, A. Imamoglu, J. P. Marangos, Rev. Mod. Phys. 2005, 77, 633 .

${ }^{219}$ A. Kasapi, M. Jain, G. Yin, S. E. Harris, Phys. Rev. Lett. 1995, $74,2447$.

${ }^{220}$ G. P. Agrawal, J. Opt. Soc. Am. B 1988, 5, 147.

${ }^{221}$ Y. Hu, M. Lindberg, S. Koch, Phys. Rev. B 1990, 42, 1713

${ }^{222}$ M. Sugawara, H. Ebe, N. Hatori, M. Ishida, Y. Arakawa, T. Akiyama, K. Otsubo, Y. Nakata, Phys. Rev. B 2004, 69, 235332.

${ }^{223}$ J. Khurgin, J. Opt. Soc. Am. B 1989, 6, 1673

${ }^{224}$ M. Belkin, F. Capasso, A. Belyanin, D. Sivco, A. Cho, D. Oakley, C. Vineis, G. Turner, Nat. Photon. 2007, 1, 288.

${ }^{225}$ B. A. Burnett, B. S. Williams, Phys. Rev. Appl. 2016, 5, 034013.

${ }^{226}$ C. Jirauschek, H. Okeil, P. Lugli, Opt. Express 2015, 23, 1670.

${ }^{227}$ A. Vizbaras, M. Anders, S. Katz, C. Grasse, G. Boehm, R. Meyer, M. A. Belkin, M.-C. Amann, IEEE J. Quantum Electron. 2011, 47, 691 .
${ }^{228}$ P. N. Butcher, D. Cotter, The Elements of Nonlinear Optics, Vol. 9, Cambridge University Press, 1991.

${ }^{229} \mathrm{Y}$. Shen, The Principles of Nonlinear Optics, WileyInterscience, New York, 1984.

${ }^{230}$ G. Lamb, Rev. Mod. Phys. 1971, 43, 99.

${ }^{231}$ A. Maimistov, A. Basharov, S. Elyutin, Y. M. Sklyarov, Phys. Rep. 1990, 191, 1.

${ }^{232}$ M. A. Talukder, C. R. Menyuk, Appl. Phys. Lett. 2009, 95, 071109 .

${ }^{233}$ M. A. Talukder, C. R. Menyuk, Opt. Express 2010, 18, 5639.

${ }^{234}$ B. R. Mollow, Phys. Rev. 1969, 188, 1969.

${ }^{235}$ R. Bullough, P. Jack, P. Kitchenside, R. Saunders, Phys. Scr. 1979, 20,364.

${ }^{236}$ A. Kujawski, J. Mostowski, J. Opt. Soc. Am. B 1986, 3, 1700.

${ }^{237}$ J. Javaloyes, S. Balle, Freetwm: a simulation tool for semiconductor lasers, https://onl.uib.eu/Softwares/Freetwm/ 2018.

${ }^{238}$ Kintechlab, Electromagnetic Template Library, http://fdtd. kintechlab.com/en/start 2018.

${ }^{239}$ A. Deinega, T. Seideman, Phys. Rev. A 2014, 89, 022501.

${ }^{240}$ A. F. Oskooi, D. Roundy, M. Ibanescu, P. Bermel, J. Joannopoulos, S. G. Johnson, Computer Physics Communications 2010, 181, 687.

${ }^{241}$ M. Riesch, C. Jirauschek, mbsolve: An open-source solver tool for the Maxwell-Bloch equations, https://github.com/ mriesch-tum/mbsolve 2017.

${ }^{242}$ Quantopticon, Quantillion Software, https://quantopticon. co.uk, 2018

${ }^{243}$ P. D. Lax, B. Wendroff, Commun. Pure Appl. Math. 1960, 13, 217.

${ }^{244}$ H. Risken, K. Nummedal, J. Appl. Phys. 1968, 39, 4662.

${ }^{245}$ E. Isaacson, H. B. Keller, Analysis of Numerical Methods, John Wiley \& Sons, 1994.

${ }^{246}$ A. Harten, J. Comput. Phys. 1983, 49, 357.

${ }^{247}$ S. K. Godunov, Matematicheskii Sbornik 1959, 89, 271.

${ }^{248}$ P. K. Nielsen, H. Thyrrestrup, J. Mørk, B. Tromborg, Opt. Express 2007, 15, 6396 .

249 J. Xiong, M. Colice, F. Schlottau, K. Wagner, B. Fornberg, Opt. Quant. Electron. 2008, 40, 447.

${ }^{250}$ G. Demeter, Comput. Phys. Commun. 2013, 184, 1203.

${ }^{251}$ J. C. Butcher, Numerical Methods for Ordinary Differentail Equations, John Wiley, 2003.

${ }^{252}$ A. Iserles, A First Course in the Numerical Analysis of Differential Equations, Cambridge University Press, 1996.

${ }^{253}$ C. W. Gear, Numerical Initial Value Problems in Ordinary Differential Equations, Prentice Hall, 1971.

${ }^{254}$ P. Arve, P. Jänes, L. Thylén, Phys. Rev. A 2004, 69, 063809.

${ }^{255}$ B. Gross, J. T. Manassah, Opt. Lett. 1992, 17, 340

${ }^{256}$ B. Bidégaray, A. Bourgeade, D. Reignier, J. Comput. Phys. 2001, 170, 603 .

${ }^{257}$ B. Bidégaray, Numer. Methods Partial Differ. Equ. 2003, 19, 284.

${ }^{258}$ K. Yee, IEEE Trans. Antennas. Propag. 1966, 14, 302.

${ }^{259}$ S. Krishnamoorthy, M. Baskaran, U. Bondhugula, J. Ramanujam, A. Rountev, P. Sadayappan, SIGPLAN Not. 2007, 42, 235.

${ }^{260}$ M. Riesch, N. Tchipev, S. Senninger, H.-J. Bungartz, C. Jirauschek, Opt. Quant. Electron. 2018, 50, 112.

${ }^{261}$ F. Schlottau, M. Piket-May, K. Wagner, Opt. Express 2005, 13, 182 .

${ }^{262}$ Q. H. Liu, Microw. Opt. Technol. Lett. 1997, 15, 158.

${ }^{263}$ O. Saut, A. Bourgeade, J. Comput. Phys. 2006, 213, 823.

${ }^{264}$ B. Garraway, P. Knight, Phys. Rev. A 1994, 49, 1266.

${ }^{265}$ E. Hairer, S. P. Nørsett, G. Wanner, Solving Ordinary Differential Equations I 2nd ed., Springer-Verlag Berlin Heidelberg, 1993.

${ }^{266}$ G. Strang, SIAM J. Numer. Anal. 1968, 5, 506.

${ }^{267}$ J. Gallier, D. Xu, Int. J. Robot. Autom. 2003, 18, 10.

${ }^{268}$ C. Moler, C. V. Loan, SIAM Rev. 2003, 45, 3.

${ }^{269}$ D. H. Hailu, https: //arxiv. org/abs/1610. 059512016. 
${ }^{270}$ C. Weninger, N. Rohringer, Phys. Rev. A 2013, 88, 053421.

${ }^{271}$ L. Guduff, A. J. Allami, C. van Heijenoort, J.-N. Dumez, I. Kuprov, Phys. Chem. Chem. Phys. 2017, 19, 17577.

${ }^{272}$ R. Kosloff, Annu. Rev. Phys. Chem. 1994, 45, 145.

${ }^{273}$ M. Riesch, C. Jirauschek, J. Comput. Phys. 2019, 390, 290.

${ }^{274}$ S.-L. Chua, Y. Chong, A. D. Stone, M. Soljačić, J. Bravo-Abad, Opt. Express 2011, 19, 1539.

${ }^{275}$ C. Leforestier, R. H. Bisseling, C. Cerjan, M. D. Feit, R. Friesner, A. Guldberg, A. Hammerich, G. Jolicard, W. Karrlein, H.D. Meyer, N. Lipkin, O. Roncero, R. Kosloff, J. Comput. Phys. 1991, 94, 59.

${ }^{276}$ U. Peskin, R. Kosloff, N. Moiseyev, J. Chem. Phys. 1994, 100, 8849.

${ }^{277}$ J. C. Tremblay, T. Carrington Jr, J. Chem. Phys. 2004, 121, 11535.

${ }^{278}$ A. Gordon, C. Jirauschek, F. X. Kärtner, Phys. Rev. A 2006, 73, 042505

${ }^{279}$ S. Blanes, F. Casas, A. Murua, J. Chem. Phys. 2017, 146, 114109.

${ }^{280}$ L. Pierantoni, D. Mencarelli, T. Rozzi, IEEE Trans. Microw. Theory Tech. 2008, 56, 654 .

${ }^{281}$ I. Ahmed, E. H. Khoo, E. Li, R. Mittra, IEEE Antennas Wirel. Propag. Lett. 2010, 9, 914.

${ }^{282}$ I. P. Christov, M. M. Murnane, H. C. Kapteyn, Phys. Rev. A 1998, $57, \mathrm{R} 2285$.

${ }^{283}$ E. Lorin, S. Chelkowski, A. Bandrauk, Comput. Phys. Commun. 2007, 177, 908.

${ }^{284}$ Q. Chen, H. Qin, J. Liu, J. Xiao, R. Zhang, Y. He, Y. Wang, J. Comput. Phys. 2017, 349, 441.

${ }^{285}$ Y. P. Chen, W. E. Sha, L. Jiang, M. Meng, Y. M. Wu, W. C. Chew, Comput. Phys. Commun. 2017, 215, 63.

${ }^{286}$ D. Masiello, E. Deumens, Y. Öhrn, Phys. Rev. A 2005, 71, 032108.

${ }^{287}$ E. Schelew, R.-C. Ge, S. Hughes, J. Pond, J. F. Young, Phys. Rev. A 2017, 95, 063853.

${ }^{288}$ M. Wegener, D. Chemla, S. Schmitt-Rink, W. Schäfer, Phys. Rev. A 1990, 42, 5675 .

${ }^{289}$ G. Y. Slepyan, A. Magyarov, S. Maksimenko, A. Hoffmann, D. Bimberg, Phys. Rev. B 2004, 70, 045320.

${ }^{290}$ K. Dolgaleva, R. W. Boyd, Adv. Opt. Photonics 2012, 4, 1.

${ }^{291}$ C. M. Bowden, J. P. Dowling, Phys. Rev. A 1994, 49, 1514.

${ }^{292}$ K. Xia, S. Gong, C. Liu, X. Song, Y. Niu, Opt. Express 2005, $13,5913$.

${ }^{293}$ M. E. Crenshaw, C. M. Bowden, Phys. Rev. A 1996, 53, 1139.

${ }^{294}$ M. E. Crenshaw, K. U. Sullivan, C. M. Bowden, Opt. Express 1997, 1,152

${ }^{295}$ J. P. Dowling, C. M. Bowden, Phys. Rev. Lett. 1993, 70, 1421.

${ }^{296}$ A. Afanas'ev, R. Vlasov, A. Cherstvyi, J. Exp. Theor. Phys. 2000, 90, 428.

${ }^{297}$ M. Crenshaw, M. Scalora, C. M. Bowden, Phys. Rev. Lett. 1992, 68, 911.

${ }^{298}$ A. Afanas'ev, R. Vlasov, O. K. Khasanov, T. Smirnova, O. Fedotova, J. Opt. Soc. Am. B 2002, 19, 911.

${ }^{299}$ D. V. Novitsky, Phys. Rev. A 2011, 84, 013817.

${ }^{300}$ E. Paspalakis, A. Kalini, A. F. Terzis, Phys. Rev. B 2006, 73, 073305.

${ }^{301}$ Y. Mitsumori, S. Watanabe, K. Asakura, K. Seki, K. Edamatsu, K. Akahane, N. Yamamoto, Phys. Rev. B 2018, 97, 235305.

${ }^{302}$ A. E. Siegman, Lasers, University Science Books, 1986.

${ }^{303}$ N. Majer, K. Lüdge, E. Schöll, Phys. Rev. B 2010, 82, 235301.

${ }^{304}$ D. Polder, M. Schuurmans, Q. Vrehen, Phys. Rev. A 1979, 19, 1192.

${ }^{305}$ K. Wodkiewicz, Phys. Rev. A 1979, 19, 1686.

${ }^{306}$ J. Andreasen, H. Cao, J. Light. Technol. 2009, 27, 4530.

${ }^{307}$ C. Gardiner, P. Zoller, Quantum Noise: A Handbook of Markovian and Non-Markovian Quantum Stochastic Methods with Applications to Quantum Optics, Vol. 56, Springer Science \& Business Media, 2004.

${ }^{308}$ M. Lax, W. Louisell, Phys. Rev. 1969, 185, 568.

${ }^{309}$ P. Drummond, M. Raymer, Phys. Rev. A 1991, 44, 2072.
${ }^{310}$ E. Gehrig, O. Hess, Phys. Rev. A 2002, 65, 033804.

${ }^{311}$ J. E. Kim, E. Malic, M. Richter, A. Wilms, A. Knorr, IEEE J. Quantum Electron. 2010, 46, 1115.

${ }^{312}$ S. Wilkinson, B. Lingnau, J. Korn, E. Schöll, K. Lüdge, IEEE J. Sel. Top. Quantum Electron. 2013, 19, 1900106.

${ }^{313}$ H. F. Hofmann, O. Hess, Phys. Rev. A 1999, 59, 2342.

${ }^{314} \mathrm{Q}$. Vu, H. Haug, O. Mücke, T. Tritschler, M. Wegener, G. Khitrova, H. Gibbs, Phys. Rev. Lett. 2004, 92, 217403.

${ }^{315}$ B. Witzigmann, V. Laino, M. Luisier, U. Schwarz, G. Feicht, W. Wegscheider, K. Engl, M. Furitsch, A. Leber, A. Lell, V. Härle, Appl. Phys. Lett. 2006, 88, 021104.

${ }^{316}$ F. Rossi, E. Molinari, Phys. Rev. Lett. 1996, 76, 3642

${ }^{317}$ D. H. Marti, M. Dupertuis, B. Deveaud, IEEE J. Quantum Electron. 2005, 41, 848.

${ }^{318}$ D. Golde, T. Meier, S. W. Koch, Phys. Rev. B 2008, 77, 075330.

${ }^{319}$ T. Stroucken, J. Grönqvist, S. Koch, J. Opt. Soc. Am. B 2012, 29, A86.

${ }^{320}$ M. Hirtschulz, F. Milde, E. Malić, S. Butscher, C. Thomsen, S. Reich, A. Knorr, Phys. Rev. B 2008, 77, 035403.

${ }^{321}$ I. Waldmueller, W. W. Chow, E. W. Young, M. C. Wanke, IEEE J. Quantum Electron. 2006, 42, 292.

${ }^{322}$ C. Jirauschek, P. Tzenov, Opt. Quant. Electron. 2017, 49, 414.

${ }^{323}$ M. S. Vitiello, G. Scamarcio, V. Spagnolo, B. S. Williams, S. Kumar, Q. Hu, J. L. Reno, Appl.Phys. Lett. 2005, 86, 111115.

${ }^{324}$ R. Nelander, A. Wacker, J. Appl. Phys. 2009, 106, 063115.

${ }^{325}$ D. Dietze, A. Benz, G. Strasser, K. Unterrainer, J. Darmo, Opt. Express 2011, 19, 13700.

${ }^{326}$ Y. Wang, A. Belyanin, Opt. Express 2015, 23, 4173.

${ }^{327}$ T. Ando, J. Phys. Soc. Jpn. 1978, 44, 765.

${ }^{328}$ T. Unuma, M. Yoshita, T. Noda, H. Sakaki, H. Akiyama, J. Appl. Phys. 2003, 93, 1586.

${ }^{329}$ V.-M. Gkortsas, C. Wang, L. Kuznetsova, L. Diehl, A. Gordon, C. Jirauschek, M. A. Belkin, A. Belyanin, F. Capasso, F. X. Kärtner, Opt. Express 2010, 18, 13616.

${ }^{330}$ A. K. Wójcik, P. Malara, R. Blanchard, T. S. Mansuripur, F. Capasso, A. Belyanin, Appl. Phys. Lett. 2013, 103, 231102.

${ }^{331}$ D. Revin, M. Hemingway, Y. Wang, J. Cockburn, A. Belyanin, Nat. Commun. 2016, 7, 11440.

${ }^{332}$ L. Columbo, S. Barbieri, C. Sirtori, M. Brambilla, Opt. Express 2018, 26, 2829.

${ }^{333}$ N. N. Vuković, J. Radovanović, V. Milanović, D. L. Boiko, IEEE J. Sel. Top. Quantum Electron. 2017, 23, 1200616.

${ }^{334}$ J. Khurgin, Y. Dikmelik, A. Hugi, J. Faist, Appl. Phys. Lett. 2014, 104, 081118.

${ }^{335}$ G. Villares, J. Faist, Opt. Express 2015, 23, 1651.

${ }^{336} \mathrm{~J}$. Bai, H. Wang, Q. Wang, D. Zhou, K. Q. Le, B. Wang, IEEE J. Quantum Electron. 2016, 52, 1.

${ }^{337}$ R. C. Iotti, F. Rossi, EPL 2016, 112, 67005.

${ }^{338}$ S. Butscher, J. Förstner, I. Waldmüller, A. Knorr, Phys. Rev. B 2005, 72, 045314.

${ }^{339}$ I. Savić, N. Vukmirović, Z. Ikonić, D. Indjin, R. W. Kelsall, P. Harrison, V. Milanović, Phys. Rev. B 2007, 76, 165310.

${ }^{340}$ D. Burghoff, T.-Y. Kao, N. Han, C. W. I. Chan, X. Cai, Y. Yang, D. J. Hayton, J.-R. Gao, J. L. Reno, Q. Hu, Nature Photon. $\mathbf{2 0 1 4}, 8,462$.

${ }^{341}$ N. S. Wingreen, C. A. Stafford, IEEE J. Quantum Electron. 1997, 33, 1170.

${ }^{342}$ E. Zibik, T. Grange, B. Carpenter, N. Porter, R. Ferreira, G. Bastard, D. Stehr, S. Winnerl, M. Helm, H. Liu, M. S. Skolnick, L. R. Wilson, Nat. Mater. 2009, 8, 803.

${ }^{343}$ N. Zhuo, F. Q. Liu, J. C. Zhang, L. J. Wang, J. Q. Liu, S. Q. Zhai, Z. G. Wang, Nanoscale Res. Lett. 2014, 9, 144.

${ }^{344}$ E. Gehrig, O. Hess, C. Ribbat, R. Sellin, D. Bimberg, Appl. Phys. Lett. 2004, 84, 1650.

${ }^{345}$ C. Sailliot, V. Voignier, G. Huyet, Opt. Commun. 2002, 212, 353.

${ }^{346}$ J. Mukherjee, J. G. McInerney, Phys. Rev. A 2009, 79, 053813.

${ }^{347}$ I. E. Protsenko, A. V. Uskov, O. Zaimidoroga, V. Samoilov, E. P. O'Reilly, Phys. Rev. A 2005, 71, 063812. 
${ }^{348}$ M. Kulkarni, O. Cotlet, H. E. Türeci, Phys. Rev. B 2014, 90, 125402.

${ }^{349}$ E. Waks, D. Sridharan, Phys. Rev. A 2010, 82, 043845.

${ }^{350}$ G. T. Adamashvili, C. Weber, A. Knorr, N. T. Adamashvili, Phys. Rev. A 2007, 75, 063808.

${ }^{351}$ L. Schneebeli, T. Feldtmann, M. Kira, S. W. Koch, N. Peyghambarian, Phys. Rev. A 2010, 81, 053852.

${ }^{352}$ W. W. Chow, H. Schneider, M. Phillips, Phys. Rev. A 2003, 68,053802 .

${ }^{353}$ B. Bidégaray-Fesquet, K. Keita, J. Math. Phys. 2014, 55, 021501.

${ }^{354}$ P. Bardella, L. L. Columbo, M. Gioannini, Opt. Express 2017, 25, 26234.

${ }^{355}$ T. R. Nielsen, P. Gartner, F. Jahnke, Phys. Rev. B 2004, 69, 235314.

${ }^{356}$ H. H. Nilsson, J.-Z. Zhang, I. Galbraith, Phys. Rev. B 2005, 72, 205331

${ }^{357}$ T. Koprucki, A. Wilms, A. Knorr, U. Bandelow, Opt. Quant. Electron. 2011, 42, 777.

${ }^{358}$ B. Lingnau, K. Lüdge, W. W. Chow, E. Schöll, Phys. Rev. E 2012, 86, 065201.

${ }^{359}$ D. Hadass, A. Bilenca, R. Alizon, H. Dery, V. Mikhelashvili, G. Eisenstein, R. Schwertberger, A. Somers, J. P. Reithmaier, A. Forchel, M. Calligaro, S. Bansropun, M. Krakowski, IEEE J. Sel. Top. Quant. 2005, 11, 1015.

${ }^{360}$ N. Yasuoka, K. Kawaguchi, H. Ebe, T. Akiyama, M. Ekawa, S. Tanaka, K. Morito, A. Uetake, M. Sugawara, Y. Arakawa, Appl. Phys. Lett. 2008, 92, 101108.

${ }^{361}$ M. van der Poel, E. Gehrig, O. Hess, D. Birkedal, J. M. Hvam, IEEE J. Quantum Electron. 2005, 41, 1115.

${ }^{362}$ A. Valavanis, L. Lever, C. Evans, Z. Ikonić, R. Kelsall, Phys. Rev. B 2008, 78, 035420.

${ }^{363}$ S. M. Barnett, S. Stenholm, Phys. Rev. A 2001, 64, 033808.

${ }^{364}$ G. Adamashvili, D. Kaup, A. Knorr, C. Weber, Phys. Rev. A 2008, 78, 013840.

${ }^{365} \mathrm{X} . \mathrm{Hu}, \mathrm{W}$. Pötz in Coherent Control in Atoms, Molecules, and Semiconductors, W. Pötz, W. Schroeder (Eds.), Springer, 1999, pp. 127-145.

${ }^{366}$ E. Laine, K. Luoma, J. Piilo, J. Phys. B 2012, 45, 154004.

${ }^{367}$ E.-M. Laine, J. Piilo, H.-P. Breuer, Phys. Rev. A 2010, 81, 062115.

${ }^{368}$ X.-M. Lu, X. Wang, C. Sun, Phys. Rev. A 2010, 82, 042103.

${ }^{369}$ J.-S. Tang, C.-F. Li, Y.-L. Li, X.-B. Zou, G.-C. Guo, H.-P. Breuer, E.-M. Laine, J. Piilo, EPL 2012, 97, 10002.

${ }^{370}$ D. Chruściński, S. Maniscalco, Phys. Rev. Lett. 2014, 112, 120404.

${ }^{371}$ A. G. Redfield, IBM J. Res. Dev. 1957, 1, 19.

${ }^{372}$ R. K. Wangsness, F. Bloch, Phys. Rev. 1953, 89, 728.

${ }^{373}$ R. S. Whitney, J. Phys. A 2008, 41, 175304.

${ }^{374}$ D. Egorova, M. Thoss, W. Domcke, H. Wang, J. Chem. Phys. 2003, 119, 2761.

${ }^{375}$ I. Knezevic, B. Novakovic, J. Comput. Electron. 2013, 12, 363.

${ }^{376}$ C. Weber, A. Wacker, A. Knorr, Phys. Rev. B 2009, 79, 165322.

${ }^{377}$ E. Vaz, J. Kyriakidis, J. Phys. Conf. Ser. 2008, $107,012012$.

${ }^{378}$ A. Pan, B. A. Burnett, C. O. Chui, B. S. Williams, Phys. Rev. B 2017, 96, 085308.

${ }^{379}$ A. Suárez, R. Silbey, I. Oppenheim, J. Chem. Phys. 1992, 97, 5101.

${ }^{380}$ H.-P. Breuer, Phys. Rev. A 2007, 75, 022103.

${ }^{381}$ A. A. Budini, Phys. Rev. E 2005, 72, 056106.

\section{BIOGRAPHIES}

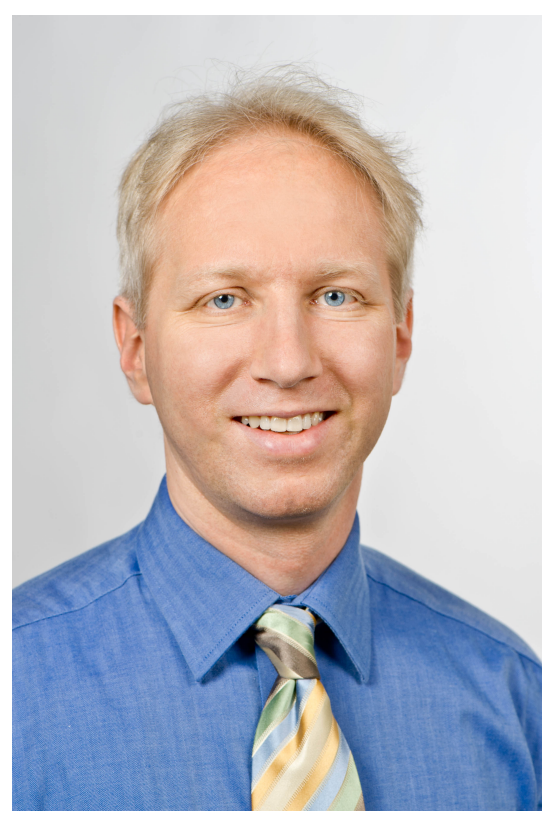

Christian Jirauschek received the Dipl.-Ing. and Doctoral degrees in Electrical Engineering from Universität Karlsruhe, Karlsruhe, Germany, in 2000 and 2004, respectively. From 2002 to 2005, he was with the Massachusetts Institute of Technology (MIT). He then joined the Institute of Nanoelectronics, Technical University of Munich (TUM), Munich, Germany, where, starting from 2007 he headed an independent junior research group within the Emmy Noether Program of the Deutsche Forschungsgemeinschaft (DFG). In 2015, he was appointed Heisenberg professor of Computational Photonics at TUM. His research interests include modeling in the areas of photonics and nanoelectronics.

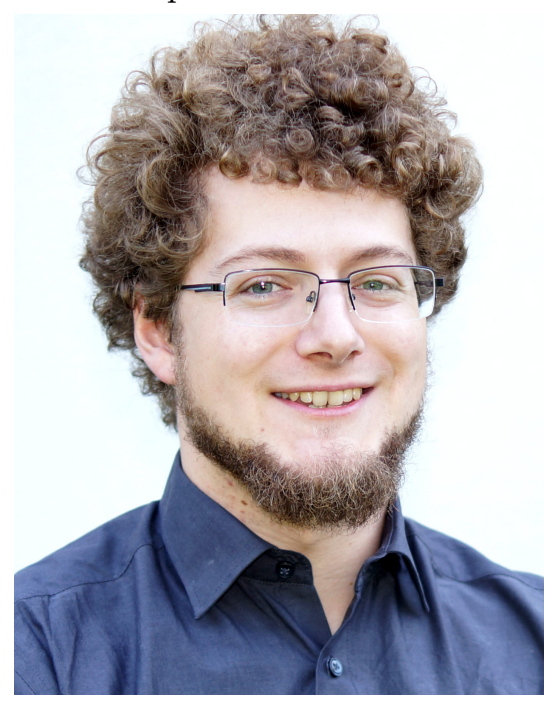

Michael Riesch received the B.Sc. and M.Sc. degrees in Electrical and Computer Engineering from the Technical University of Munich (TUM), Munich, Germany, in 2012 and 2015, respectively. He continued his studies at TUM and received the M.Sc. degree in Computa- 
tional Science and Engineering in 2016. He then joined the Computational Photonics group at TUM as research assistant. His research activities focus on the dynamical simulations of quantum cascade lasers and include numerical methods and high performance computing.

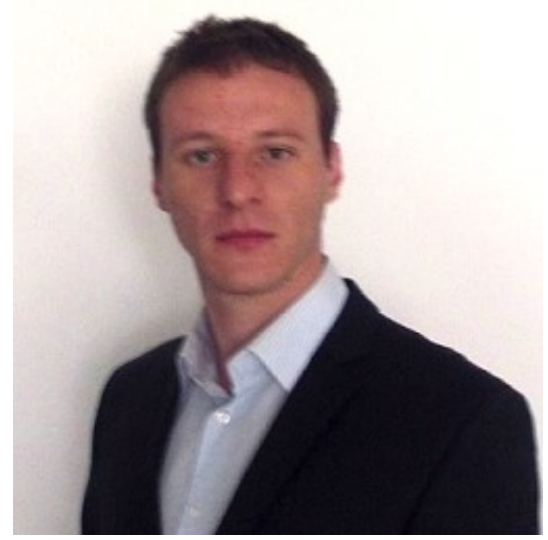

Petar Tzenov received the B.S. degree in Applied Mathematics from Sofia University (St. "Kliment Ohrisdski"), Sofia, Bulgaria, in 2012. He received the M.S. degree in Computational Science and Engineering from the Technical University of Munich (TUM), Munich, Germany, in 2014, where between 2015 and 2018, as a member of the Computational Photonics group, he pursued a Ph.D. degree in the areas of laser physics and nonlinear optics. His research interests include classical and quantum optics, quantum electronics, and computational physics, as well as high-performance computing. 